\title{
PNNL-- 11558
}

\section{Research Progress Report 1994-1995 \\ Theory, Modeling and Simulation}

\section{MASTER}

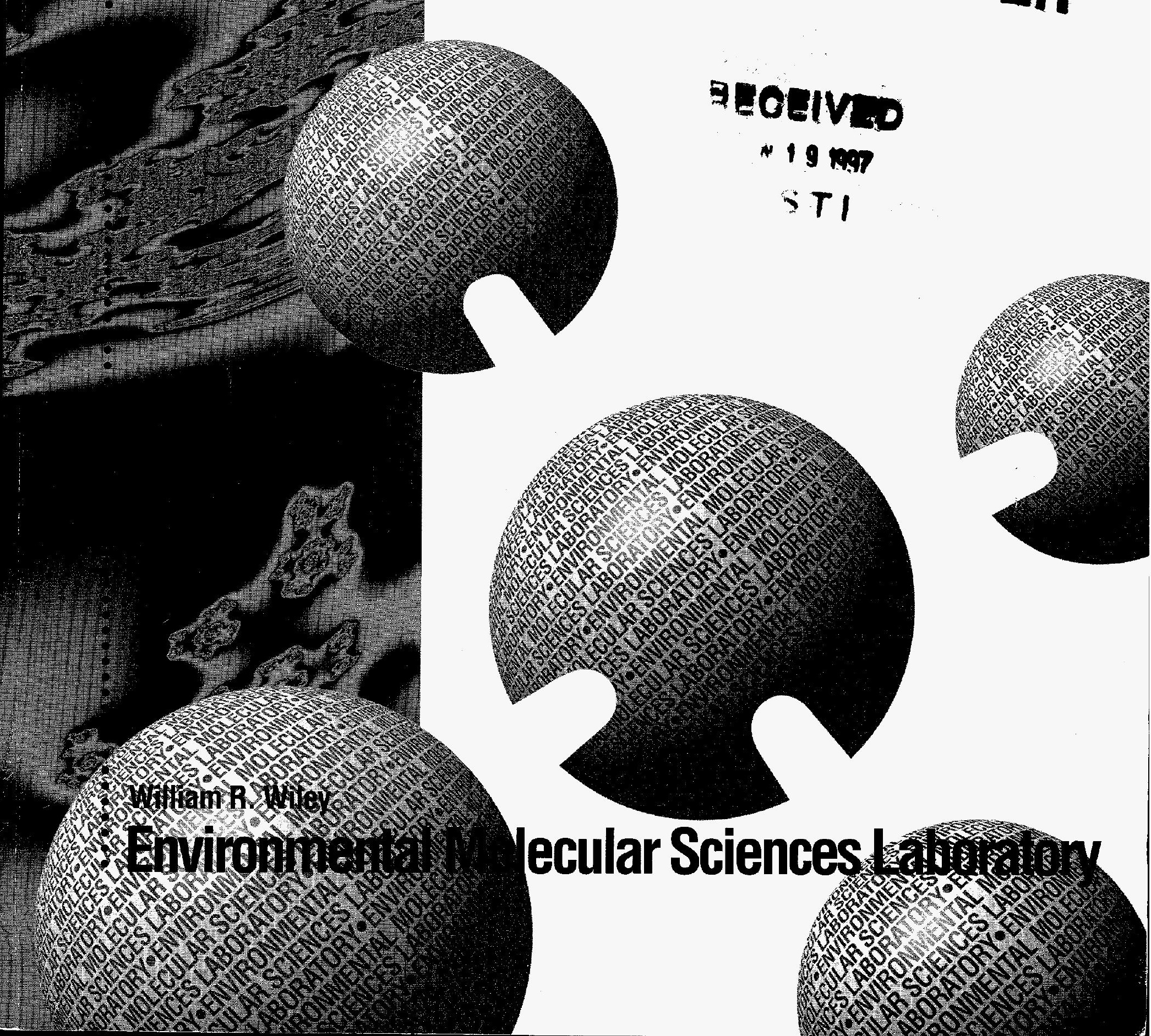




\title{
DISCLAIMER
}

This report was prepared as an account of work sponsored by an agency of the United States Government. Neither the United States Government nor any agency thereof, nor Battelle Memorial Institute, nor any of their employees, makes any warranty, express or implied, or assumes any legal liability or responsibility for the accuracy, completeness, or usefulness of any information, apparatus, product, or process disclosed, or represents that its use would not infringe privately owned rights. Reference herein to any specific commercial product, process, or service by trade name, trademark, manufacturer, or otherwise does not necessarily constitute or imply its endorsement, recommendation, or favoring by the United States Government or any agency thereof, or Battelle Memorial Institute. The views and opinions of authors expressed herein do not necessarily state or reflect those of the United States Government or any agency thereof.

\author{
PACIFIC NORTHWEST NATIONAL LABORATORY \\ operated by \\ BATTELLE \\ for the \\ UNITED STATES DEPARTMENT OF ENERGY \\ under Contract DE-AC06-76RLO 1830
}

Printed in the United States of America

Available to DOE and DOE contractors from the

Office of Scientific and Technical Information, P.O. Box 62, Oak Ridge, TN 37831; prices available from (615) $576-8401$.

Available to the public from the National Technical Information Service, U.S. Department of Commerce, 5285 Port Royal Rd., Springfield, VA 22161 


\section{DISCLAMIER}

Portions of this document may be illegible in electronic image products. Images are produced from the best available original document. 
Research Progress Report 1994-1995 Theory, Modeling and Simulation

Prepared by

Bruce C. Garrett, Group Leader, Molecular Theory

David A. Dixon, Associate Director, Theory, Modeling and Simulation

Thom H. Dunning, Director, William R. Wiley

Environmental Molecular Sciences Laboratory

January 1997

Prepared for the U.S. Department of Energy under Contract DE-ACO6-76RLO 1830

Pacific Northwest National Laboratory

Operated for the U.S. Department of Energy

by Battelle Memorial Institute 


\section{Theory, Modeling, and Simulation}

Environmental Molecular Sciences Laboratory

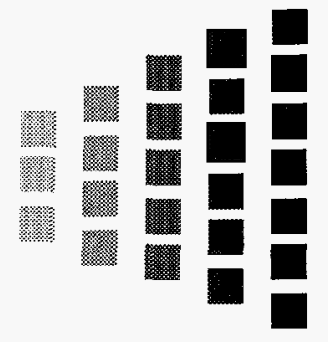

The mission of the Theory, Modeling, and Simulation program in the Environmental Molecular Sciences Laboratory is to establish state-of-the-art research efforts in computational molecular science, combining the elements of theoretical and computational chemistry, materials science, condensed matter physics, and molecular biology with computer science and applied mathematics and advanced computing technologies, to help provide comprehensive solutions to the environmental problems of the U.S. Department of Energy and the nation.

Developing the knowledge base needed to address the environmental restoration issues of the U.S. Department of Energy (DOE) requires a fundamental understanding of molecules and their interactions in isolation (gas phase), in liquids, on surfaces, and at interfaces and in condensed phases. To meet these needs, the Pacific Northwest National Laboratory (PNNL) has established the Environmental Molecular Sciences Laboratory (EMSL). In April 1994, construction began on the new EMSL, a collaborative research facility devoted to advancing our understanding of environmental molecular science. Research in the Theory, Modeling, and Simulation (TM\&S) program, which is one of six research directorates in the EMSL, will play a critical role in understanding molecular processes important in restoring DOE's research, development, and production sites, including understanding the migration and reactions of contaminants in soils and ground water, developing processes for isolation and processing of pollutants, developing improved materials for waste storage, understanding the enzymatic reactions involved in the biodegradation of contaminants, and understanding the interaction of hazardous chemicals with living organisms.

The research objectives of the TM\&S program are fivefold:

- to apply available electronic structure and dynamics techniques to study fundamental molecular processes involved in the chemistry of natural and contaminated systems

- to extend current electronic structure and dynamics techniques to treat molecular systems of future importance and to develop new techniques for addressing problems that are computationally intractable at present

- to apply available molecular modeling techniques to simulate molecular processes occurring in the multi-species, multi-phase systems characteristic of natural and polluted environments

- to extend current molecular modeling techniques to treat ever more complex molecular systems and to improve the reliability and accuracy of such simulations.

- to develop technologies for advanced parallel architectural computer systems.

The Theory, Modeling, and Simulation program contains a number of fundamental research efforts: Molecular Theory and Modeling, Solid State Theory and Modeling, Biomolecular Modeling and Simulation, High Performance Computational Chemistry, and Computational Chemistry Environment.

The Molecular Theory and Modeling activity is directed to understanding elementary molecular processes in both the gas and condensed phases with a focus on cluster chemistry, solution chemistry, and the liquid-liquid and liquid-solid 
interfaces. The Solid State Theory activity extends existing theoretical models of surfaces and materials to soil minerals (oxides, carbonates, clays) and catalysts (oxides and zeolites). The Biomolecular Modeling and Simulation activity focuses on the development and application of techniques to model biochemical systems, specifically biodegradative enzymes and proteins.

The computing demands of research in Theory, Modeling, and Simulation are substantial and are growing dramatically as new projects involving molecular systems of increasing size and complexity are undertaken, new staff are added to the program, and extensive collaborative projects are initiated. To provide for the computing needs of this effort, TM\&S will develop and manage the Molecular Science Computing Facility (MSCF) as a collaborative research facility in the EMSL. The computer hardware in the MSCF will provide state-of-the-art high performance computational and visualization capabilities for molecular simulations, while enabling the evaluation and exploitation of promising new computer technologies.

Software development must keep pace with hardware advances if we are to obtain maximum benefit from the computers installed in the MSCF. To ensure the parallel, integrated development of the software and hardware for the MSCF, a Molecular Science Software effort has been established in the TM\&S program. The Molecular Science Software effort contains two research and development activities: High Performance Computational Chemistry (HPCC) and Computational Chemistry Environment (CCE). The first of these efforts is directed to the development of a new generation of molecular modeling software that takes full advantage of the continuing rapid advances in computer technology, especially massively parallel computers. The CCE group was established in order to maximize the use of advanced parallel computers, provide efficient means for dealing with large amounts of data, and provide userfriendly interfaces for data preparation and analysis. 


\section{Contents}

Theory, Modeling, and Simulation $\ldots \ldots \ldots \ldots$ ii

Organization and Staff $\ldots \ldots \ldots \ldots \ldots \ldots$

Major Events of 1994-1995 $\ldots \ldots \ldots \ldots \ldots \ldots \ldots \ldots \ldots \ldots \ldots \ldots$

Honors, Awards, and Appointments $\ldots \ldots \ldots \ldots$ xv

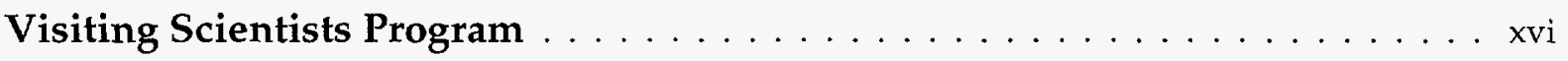

Seminar Program $\ldots \ldots \ldots \ldots \ldots \ldots \ldots \ldots \ldots \ldots \ldots \ldots \ldots \ldots \ldots$

\section{Research Highlights}

\section{Molecular Theory and Modeling}

Development of Electronic Structure Methods

Intrinsic Errors in $A b$ Initio Calculations: The Dissociation Energy of $\mathrm{N}_{2}$

K. A. Peterson and T. H. Dunning, Jr. . . . . . . . . . . . . . . . 1-1

The Accurate Calculation of Excited Electronic States: The Singlet-Triplet Splittings of $\mathrm{C}_{2}, \mathrm{CN}^{+}, \mathrm{BN}$, and $\mathrm{BO}^{+}$

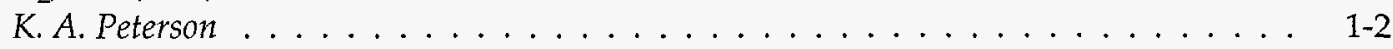

Accurate $A b$ Initio Force Fields and Vibrational Spectra: The Dipole Moment Function of $\mathrm{HCN}$

K. A. Peterson . . . . . . . . . . . . . . . . . . . . . 1-5

$A b$ Initio Studies of Magnetic Hyperfine and Electrostatic Properties of Small Molecules

D. F. Feller, E. D. Glendening, K. A. Peterson, R. J. Miller, and E. A. McCullough, Jr . . . 1-6

Benchmarking Electronic Structure Software and Hardware

D. F. Feller and R. A. Kendall . . . . . . . . . . . . . . . . . . . . 1-6

Development of Reaction Rate Theories

Variational Transition State Theory for Activated Chemical Reactions in Solution

B. C. Garrett and G. K. Schenter . . . . . . . . . . . . . . . . . . . 1-7

Tunneling in the Presence of a Bath: A Generalized Transition State Theory Approach

D. G. Truhlar, Y.-P. Liu, G. K. Schenter, and B. C. Garrett . . . . . . . . . . . . . . 1-8

Nonequilibrium Solvation for an Aqueous-Phase Reaction: Kinetic Isotope Effects for the Addition of Hydrogen to Benzene

B. C. Garrett and G. K. Schenter . . . . . . . . . . . . . . . . . . . . . 1-8

Accurate Calculation of Quantum Mechanical Reaction Rates at Low Temperatures

G. K. Schenter, M. Messina, and B. C. Garrett . . . . . . . . . . . . . . . . . 1-10

Reversible Work Transition State Theory and Its Application to the Dissociative Adsorption of Hydrogen

G. K. Schenter, G. Mills, and H. Jonsson . . . . . . . . . . . . . . . . 1-10 
The Influence of Solvent on the Rate of Chemical Reactions in the Energy-Diffusion Regime

G. K. Schenter, S. K. Reese, and S. C. Tucker . . . . . . . . . . . . . . . . . . . 1-11

\section{Modeling Molecular Processes in Clusters}

Variation of the Three-Body Interaction Energy Term in Different Water Trimer

Configurations

S. S. Xantheas

Comparison of Density Functional with MP2 for Cyclic Water Clusters

S. S. Xantheas . . . . . . . . . . . . . . . . . . . . . . 1-14

Investigation of Acetyl Chloride Photodissociation by Photofragment Imaging

S. Deshmukh, J. D. Myers, S. S. Xantheas, and W. P. Hess . . . . . . . . . . . . . . 1-15

Structures and Energetics of $\mathrm{F}^{-}\left(\mathrm{H}_{2} \mathrm{O}\right)_{n}, \mathrm{n}=1-3$ Clusters

S. S. Xantheas and T. H. Dunning, Jr. . . . . . . . . . . . . . . 1-16

$A b$ Initio Studies of $\mathrm{M}^{+}\left(\mathrm{H}_{2} \mathrm{O}\right)_{\mathrm{n}}$ and $\mathrm{M}^{2+}\left(\mathrm{H}_{2} \mathrm{O}\right)_{\mathrm{n}}$ Clusters

D. F. Feller, E. D. Glendening, K. A. Peterson, D. E. Woon, and R. A. Kendall . . . . . . . 1-19

Path Integral Monte Carlo Simulations of Ionic Clusters

H. Gai, L. X. Dang, G. K. Schenter, and B. C. Garrett . . . . . . . . . . . . . . . . 1-19

The Hydrogen Bond Energy of the Water Dimer

D. Feller, M. W. Feyereisen, and D. A. Dixon

Modeling Molecular Processes in Solution

Ionic Association Thermodynamics

D. E. Smith and L. X. Dang . . . . . . . . . . . . . . . . . . . . . 1-22

Solvent Effects on Metal Cation-Aromatic Interactions - A Molecular Dynamics Study

T.-M. Chang and L. X. Dang . . . . . . . . . . . . . . . . . . . . . . 1-22

Modeling Molecular Processes in Separations Chemistry

An $A b$ Initio Investigation of the Selectivity of 18-crown-6

S. E. Hill, E. D. Glendening, D. F. Feller, M. A. Thompson, and J. A. Nichols . . . . . . 1-23

Combined $A b$ Initio and Experimental Studies of Small Ether/Cation Clusters

D. F. Feller, E. D. Glendening, D. Ray, P. Armentrout, and M. Birke . . . . . . . . 1-24

Development of Hybrid Quantum Mechanical/Molecular Mechanical Force Fields for Crown-Ethers

M. A. Thompson, E. D. Glendening, and D. Feller

A Hybrid Quantum Mechanical/Molecular Mechanical Method that Includes MM

Polarization: Application to Excited States of the Primary Donor of the Bacterial

Photosynthetic Reaction Center

M. A. Thompson and G. K. Schenter . . . . . . . . . . . . . . . . . . . . . 1-26

Free Energy of Association of the $\mathrm{K}^{+}: 18$-Crown-6 Complex in Water: A Molecular

Dynamics Study

L. X. Dang . . . . . . . . . . . . . . . . . . . . . . . . . . . . . 1-27

Mechanism and Thermodynamics of Ion Selectivity in Aqueous Solutions of

18-Crown-6 Ether: A Molecular Dynamics Study

L. X. Dang . . . . . . . . . . . . . . . . . . . . . . . . . . . 1-28

Modeling Molecular Processes at Aqueous Interfaces

Investigation of the Liquid-Vapor Interface of Water via Molecular Dynamics

R. S. Taylor, L. X. Dang, and B. C. Garrett . . . . . . . . . . . . . . . . . . . . . 1-29 
Solvation of the $\mathrm{Cs}^{+}$Ion at the Liquid-Vapor Interface of Water

L. X. Dang, R. S. Taylor, and B. C. Garrett . . . . . . . . . . . . . . . . . 1-30

Molecular Dynamics Studies of Processes at Liquid-Liquid Interfaces

T.-M. Chang and L. X. Dang . . . . . . . . . . . . . . . . . . . . . 1-31

Physics and Chemistry of Structurally Simple Oxides

M. I. McCarthy, A. C. Hess, J. B. Nicholas, C. A. Scamehorn, M. R. Chacon-Taylor,

M. Leslie, N. Harrison, and M. Towler . . . . . . . . . . . . . . . . . . 1-31

$\mathrm{TiO}_{2}$ Surface Chemistry and Physics

M. I. McCarthy, M. A. Henderson, K. Briggman . . . . . . . . . . . . . . . 1-33

Laser Ablation of Molecular-Ionic Crystals

M. I. McCarthy, K. A. Peterson, W. P. Hess, T. M. Orlando, and R. A. Bradley . . . . . 1-35

Heterogeneous Interface Chemistry of Ices

M. I. McCarthy, R. D. Poshusta, and S. George . . . . . . . . . . . . . 1-36

Physics of Polymer Interfaces

M. I. McCarthy, R. A. Kendall, K. deDing, D. A. Dixon, and J. Glas . . . . . . . . 1-37

A Semiempirical Molecular Dynamics Methodology for Simulating Covalently

Bonded Materials: Si, C, SiC, and Se

L. R. Corrales, A. Chen, and S. M. Slate . . . . . . . . . . . . . . . 1-38

\section{Solid State Theory and Modeling}

Development of Methodology for Modeling Solid State Systems

Development Status of the Periodic Gaussian Basis Density Functional Methods

A. C. Hess, D. G. Clerc, Z. Lin, J. E. Jaffe, H. L. Taylor, and J. A. Nichols . . . . . . . . . 2-1

A Multicenter Integration Scheme for Electronic Structure Calculations of Periodic and Non-Periodic Polyatomic Systems

Z. Lin and A. C. Hess . . . . . . . . . . . . . . . . . . . . .

Chemistry and Physics of Materials

CO Adsorption on the Nonpolar (1010) Surface of $\mathrm{ZnO}$

J. E. Jaffe and A. C. Hess . . . . . . . . . . . . . . . . . . 2-5

Periodic Hartree-Fock Study of $\mathrm{Li}_{\mathrm{x}} \mathrm{TiS}_{2}, 0 \leq \mathrm{x} \leq 1$

D. G. Clerc, R. D. Poshusta, and A. C. Hess . . . . . . . . . . . . 2-5

Periodic Hartree-Fock Pseudopotential Study of $\mathrm{Li}_{\mathrm{x}} \mathrm{Cd}_{2} \mathrm{P}_{2} \mathrm{~S}_{6}, \mathrm{x}=0,1,2$, and 3

D. G. Clerc, R. D. Poshusta, and A. C. Hess . . . . . . . . . . . . 2-6

Chemistry and Physics of Catalysis

Local Density Functional Studies of [T]-ZSM-5, T $=\mathrm{Si}, \mathrm{B}, \mathrm{Al}, \mathrm{Ga}$, and $\mathrm{Fe}$

M. S. Stave and J. B. Nicholas . . . . . . . . . . . . . . . . . . .

Comparison of Density Functional and $A b$ Initio Methods for the Determination of

Transition State Geometries and Energetics

Q. Zhao and J. B. Nicholas ... . . . . . . . . . . . . . . 2-8

Unified NMR and DFT Study of Hammett Bases in Acidic Zeolites

J. F. Haw, J. B. Nicholas, L. W. Beck, T. R. Krawietz, and D. B. Ferguson . . . . . . . . 2-9

DFT Study of the Protonation of Alkenes by Zeolites

Q. Zhao and J. B. Nicholas. . . . . . . . . . . . . . . . . . . . . . . 2-9

NMR and DFT Study of H-D Exchange in Benzene on Acidic Zeolites

L.W. Beck, J. B. Nicholas, and J. F. Haw 


\section{Biomolecular Modeling and Simulation}

Understanding Enzyme Structure-Function-Dynamics Relations

Domain-Domain Dynamics: T4 Lysozyme

G. E. Arnold, J. I. Manchester, B. D. Townsend, and R. L. Ornstein . . . . . . . . . . . 3-2

Crystal Packing Forces and Their Effects on Protein Conformation and Dynamics: T4

Lysozyme

G. E. Arnold and R. L. Ornstein . . . . . . . . . . . . . . . . . . . . . . .

Unexpectedly Large Dynamic Motions Across the 'Mouth' of the Active Site of

Cytochrome P450BM3

M. D. Paulsen, T. B. Nguyen, and R. L. Ornstein

Understanding and Improving Enzyme Dehalogenation

Dehalogenation of Pentachloroethane by Cytochrome P450cam: Why F87W Is Faster than Wild-Type

J. I. Manchester and R. L. Ornstein . . . . . . . . . . . . . . . . . . 3-6

Toward Rational Redesign of Haloalkane Dehalogenase

G. E. Arnold and R. L. Ornstein . . . . . . . . . . . . . . . . . . . . . . . .

Methods Validation and Improvement for Enzyme Simulations

Prediction and Experimental Verification of Product Profiles: Cytochrome P450cam Hydroxylation of Norcamphor

M. D. Paulsen, G. E. Arnold, R. L. Ornstein, P. J. Loida, and S. G. Sligar . . . . . . .

Extremozymes - Toward Improving Nonaqueous Functionality: Subtilisin Y.-J. Zheng and R. L. Ornstein . . . . . . . . . . . . . . . . . . . .

Modeling a New Reductive Dehalogenase and Experimental Collaborations in EMSL R. J. Martinez, L. Xun, M. A. Kennedy, and R. L. Ornstein . . . . . . . . . . . . . . . 3-10

\section{High Performance Computational Chemistry}

\section{Software Development Tools}

Message Passing

R. J. Harrison and J. Nieplocha . . . . . . . . . . . . . . . . . . . . . 4 4-2

Memory Allocation

D. E. Bernholdt, R. J. Harrison, R. J. Littlefield, and G. S. Thomas . . . . . . . . . . 4-2

Global Arrays

R. J. Harrison and J. Nieplocha ... . . . . . . . . . . . . . 4-3

Distributed Matrices and Linear Algebra

D. Elwood, G. I. Fann, and R. J. Littlefield . . . . . . . . . . . . . . . 4-5

Run Time Database

R. J. Harrison and R. A. Kendall . . . . . . . . . . . . . . . . . . 4 4-7

Parallel Computational Chemistry Software

Common Software Components

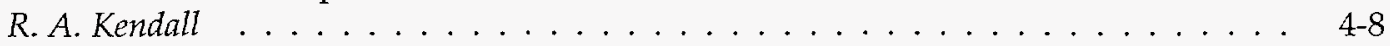

Self-Consistent Field Hartree-Fock

H. A. Früchtl, R. A. Kendall, R. J. Harrison, and A. T. Wong . . . . . . . . . . . . . 4-9

Direct Four-Index Transformation

R. J. Harrison, A. P. Rendell, and A. T. Wong . . . . . . . . . . . . . . 4-12 
Direct Multiconfiguration Self-Consistent Field

R. J. Harrison and A. T. Wong . . . . . . . . . . . . . . . . . . . . . 4-13

Møller-Plesset Perturbation Theory

D. E. Bernholdt and R. J. Harrison . . . . . . . . . . . . . . . . . . 4-13

Density Functional Theory

J. A. Anchell, E. Apra', A. C. Hess, J. E. Jaffe, J. A. Nichols, and H. L. Taylor . . . . . 4-16

Collaboration in Molecular Science Software:

Daresbury Laboratory - Pacific Northwest National Laboratory

Advanced Correlated Methods

D. E. Bernholdt, R. J. Harrison, A. P. Rendell, and A. T. Wong . . . . . . . . . . . 4-18

Hybrid QM/MM Methods

M. F. Guest, X. Long, J. B. Nicholas, and P. Sherwood . . . . . . . . . . . . . . . 4-18

Electronic Structure of Periodic Solids: The Parallelization of CRYSTAL92

E. Aprà and V. R. Saunders . . . . . . . . . . . . . . . . . . . . . 4 4-20

Molecular Dynamics Simulations

R. Kutteh, J. B. Nicholas, and W. Smith . . . . . . . . . . . . . . . . . . 4-20

Applications to Prototypical or Model Problems in Chemistry

An Extended Basis Set $A b$ Initio Study of $\mathrm{Li}^{+}(\mathrm{H} 20)_{n}, \mathrm{n}=1-6$

D. F. Feller, E. D. Glendening, R. A. Kendall, and K. A. Peterson . . . . . . . . . . 4-24

Theoretical Studies of Inorganic and Organometallic Reaction Mechanisms: Hydrogen

Exchange in the B-agostic Ethylene Complex of Cyclopentadienyl Rhodium

M. F. Guest, M. B. Hall, Z. Lin, and P. Sherwood . . . . . . . . . . . . . . . . . 4-24

Models for the Molybdenum (VI/V) Centers of the Molybdenum Hydroxylases and

Related Enzymes. Geometry, Electronic Structure, and EPR g-Tensor Predictions

from $A b$ Initio and Zindo Molecular Orbital Studies

E. McCullough, J. A. Nichols, G. Peng, and J. Spence

Benchmark Calculations with Correlated Molecular Wave Functions. III. Configuration Interaction Calculations on First Row Homonuclear Diatomics

K. A. Peterson, R. A. Kendall, and T. H. Dunning, Jr. . . . . . . . . . . . . . . 4-25

Ionization Potentials, Electron Affinities, and Potential Energy Curves Determined from the Multiconfigurational Spin Tensor Electron Propagator Method (MCSTEP) J. T. Golab, D. Heryadi, J. A. Nichols, and D. L. Yeager . . . . . . . . . . . . . 4-25

Application of Combined Density Functional Theory/Molecular Mechanics to Small Water Clusters: A Test of Methodology

X. P. Long, J. B. Nicholas, M. F. Guest, and R. L. Ornstein . . . . . . . . . . . . . . . 4-26

Molecular Mechanics Parameters for Fluorine-Substituted Methanes from Ab Initio Quantum Calculations J. L. Anchell and B. J. Palmer . . . . . . . . . . . . . . . . . . . . . . 4 4-26

Application of a Systematic Sequence of Basis Sets to the NMR Shielding of $\mathrm{CH}_{4}$ and $\mathrm{SiH}_{4}$

D. E. Bernholdt and J. B. Nicholas . . . . . . . . . . . . . . . . . . . 4-27

Weakly Bound van der Waals Complexes: The Interaction of Ar with NNO H. A. Früchtl, R. A. Kendall, and A. T. Wong . . . . . . . . . . . . . . . . . 4-28

Density Functional Calculations of Surface Defects on $\mathrm{MgO}(001)$ : Candidate Structures for Positive Ion Photo-Desorption

J. T. Dickinson, A. S. Gibson, J. P. Lafemina, J. A. Nichols, and H. L. Taylor . . . . . . 4 4-28 


\section{Computational Chemistry Environment}

Extensible Computational Chemistry Environment

Architecture for Molecular Modeling Applications

Object-Oriented Experiment Management and Computational Chemistry Data Model

T. L. Keller, K. L. Schuchardt, E. Overton, D. Feller, and D.R. Jones. . . . . . . . . . . . 5-3

Periodic Table Graphical User Interface Widget

G.D. Black

Internet Access to the EMSL Gaussian Basis Set Library

K. L. Schuchardt, D. Feller, and D. R. Jones

EMSL Graphics and Visualization Laboratory

R. May and D. R. Jones

\section{Publications and Submissions}

Publications ............................ 6.1

Other Publications $\ldots \ldots \ldots \ldots \ldots \ldots \ldots \ldots \ldots \ldots$ 


\section{Organization and Staff}

Theory, Modeling, and Simulation

Theory, Modeling, and Simulation is one of six research directorates in the Environmental Molecular Sciences Laboratory. It consists of the Associate Director's Office and four research groups. The staff, postdoctoral fellows, and graduate students in each of these groups are listed below.

\section{EMSL Director's Office}

Thom H. Dunning, Jr., Director

Sotiris S. Xantheas, Research Scientist

Tanja van Mourik, Postdoctoral Fellow

Angela K. Wilson, Postdoctoral Fellow

\section{Associate Director's Office}

David A. Dixon, Associate Director

Robert A. Eades, Program Manager

Deborah S. Baker, TM\&S Secretary

Molecular Theory and Modeling Group

Bruce C. Garrett, Group Leader/Chief Scientist

Kari L. Fausey, Group Secretary

L. Rene Corrales, Senior Research Scientist

Liem X. Dang, Senior Research Scientist

David Feller, ${ }^{1}$ Staff Scientist

Maureen I. McCarthy, Senior Research Scientist

Bruce J. Palmer, ${ }^{2}$ Senior Research Scientist

Kirk A. Peterson, ${ }^{3}$ Postdoctoral Fellow

Gregory K. Schenter, Senior Research Scientist

Mark A. Thompson, 1 Senior Research Scientist

Monique Chacon-Taylor, Postdoctoral Fellow

Tsun-Mei Chang, Postdoctoral Fellow

Ai Chen, Postdoctoral Fellow

Kurt deDing, Graduate Student

Huadong Gai, Postdoctoral Fellow

Eric Glendening, Posdtoctoral Fellow

Susan E. Hill, Postdoctoral Fellow

Xiping Long, Postdoctoral Fellow

Stephen L. Mielke, Postdoctoral Fellow

Carol A. Scamehorn, Postdoctoral Fellow

David E. Smith, Postdoctoral Fellow

Ramona S. Taylor, Postdoctoral Fellow

David E. Woon, Postdoctoral Fellow 


\section{Solid State Theory Group}

Anthony C. Hess, Project Leader/Senior Research Scientist John B. Nicholas, ${ }^{4}$ Senior Research Scientist

James L. Anchell, ${ }^{4}$ Postdoctoral Fellow Edoardo Aprá, ${ }^{4}$ Postdoctoral Fellow Daryl G. Clerc, Postdoctoral Fellow Thomas J. Godin, Postdoctoral Fellow Timothy E. Meehan, Postdoctoral Fellow Roberto Nada, Postdoctoral Fellow Julie C. White, Postdoctoral Fellow

\section{Biomolecular Modeling and Simulation Group}

Rick L. Ornstein, Staff Scientist Mark D. Paulsen, Senior Research Scientist Gregory E. Arnold, Senior Research Scientist Karol Miaskiewics, Research Scientist

Xiping Long, 5 Postdoctoral Fellow John I. Manchester, Graduate Student Osmar Norberto de Souza, Postdoctoral Fellow Robert F. Setlik, Graduate Student Yajun Zheng, Postdoctoral Fellow

\section{High Performance Computational Chemistry Group}

Martyn F. Guest, Group Leader/Senior Chief Scientist Jeffrey A. Nichols, Interim Group Leader/Staff Scientist Rebecca Wattenburger, Group Secretary

Michel Dupuis, Senior Chief Scientist Robert J. Harrison, Staff Scientist Rick A. Kendall, Staff Scientist John B. Nicholas, ${ }^{4}$ Senior Research Scientist Tjerk P. Straatsma, Senior Research Scientist

James L. Anchell, ${ }^{4}$ Postdoctoral Fellow Edoardo Aprá, 4 Postdoctoral Fellow David E. Bernholdt, Postdoctoral Fellow Miles J.O. Deegan, Postdoctoral Fellow Herbert A. Fruechtl, Postdoctoral Fellow Ramzi A. Kutteh, Postdoctoral Fellow Xiping Long, ${ }^{5}$ Postdoctoral Fellow Hugh Taylor, Postdoctoral Fellow Adrian T. Wong, Postdoctoral Fellow

\section{Computational Chemistry Environment Group}

Donald R. Jones, Group Leader Rebecca Wattenburger, Group Secretary

Curtis N. Bingham, ${ }^{6}$ Research Scientist 
Gary D. Black, ${ }^{6}$ Research Scientist

David F. Feller, ${ }^{1}$ Staff Scientist

Annie Groeninger, ${ }^{6}$ Scientist

Randy W. Heiland, ${ }^{6}$ Research Scientist

Mark E. Hinchee, ${ }^{6}$ Research Scientist

Jimmy Jou, ${ }^{6}$ Research Scientist

Thomas L. Keller, ${ }^{6}$ Research Scientist

Richard A. May, ${ }^{6}$ Scientist

Karen Shuchardt, ${ }^{6}$ Senior Research Scientist

Geoff S. Slinker, 6 Scientist

Anne K. Schur, ${ }^{6}$ Research Scientist

Mark A. Thompson, ${ }^{1}$ Senior Research Scientist

Erin N. Thornton, ${ }^{6}$ Research Engineer

Mark L. Wilkins, ${ }^{6}$ Research Scientist

Carol Winter, ${ }^{6}$ Research Scientist

Chance R. Younkin, ${ }^{6}$ Research Scientist

Stephen J. Brunelle, Graduate Student

Rupa K. Sethu, ${ }^{6}$ Consultant

1 Joint appointment between Molecular Theory and Modeling and Computational Chemistry Environment Groups.

2 Joint appointment with the Applied Mathematics Group, EMSL.

3 Joint appointment with the Washington State University.

4 Joint appointment between Solid State Theory and Modeling and High Performance Computational Chemistry Groups.

5 Joint appointment between Biomolecular Modeling and Simulation and High Performance Computational Chemistry Groups.

6 Joint appointment with Computer and Information Science, EMSL. 
The period 1994-1995 was one of transition for Theory, Modeling, and Simulation. Most significant was Thom Dunning's acceptance of his new position as the Director of the Environmental Molecular Sciences Laboratory. While a nationwide search was in progress for his replacement as Associate Director for TM\&S, Bruce Garrett took on the acting responsibilities of the Acting Associate Director. In September 1995, Dr. David A. Dixon joined TM\&S as the new Associate Director. Also, Dr. Robert A. Eades joined TM\&S as Manger of the Molecular Science Computing Facility in January 1995. Dr. Martyn Guest, HPCC Group Leader, returned to Daresbury in April 1995 and Dr. Jeffrey Nichols was appointed HPCC Group Leader in May 1995. Dr. Tjerk Straatsma joined the HPCC Group as a Senior Research Scientist in February 1995, and Dr. Michel Dupuis joined the group as a Senior Chief Scientist in December 1995.

The research programs in Theory, Modeling, and Simulation continued to evolve with three new research projects being funded by the Department of Energy.

- Cooperative Research and Development Agreement (CRADA) project entitled "Environmental Catalysis" by John B. Nicholas (PNNL) and Roy Periana (Catalytica Advanced Technologies). The purpose of this research project is to develop new, environmentally safe catalysts to replace the toxic, strong acids and bases currently used in industral processes. The current focus of the research is on new catalysts for the conversion of methane to methanol.

- CRADA project entitled "Fluropolymer Physics" by Maureen I. McCarthy (PNNL) and David A. Dixon (DuPont). This project is focused on understanding the properties of polytetrafluoroethylene and other perflourinated polymers such as those used for coatings. A combined approach involving molecular and periodic electronic structure calculations together with statistical models is being used to determine the dependence of the solid properties (e.g., stress/strain, dielectric behavior) on chemical composition and the structural characteristics of the polymer melts. The final goal of this work is to develop methods that will lead to improved understanding of the interaction of these polymers with metal and metal oxided surfaces for thin film applications.

- CRADA project entitled "Quantum Mechanical/Molecular Mechanical Methods Development with Applications to the Development of Pesticides, Dyes, and Polymers and to General Solution Photochemistry" by Mark A. Thompson and Paul Soper, Dan Kleier, and Kerwin Dobbs (DuPont). The primary purpose of this CRADA is to apply PNNL's existing Hybrid Quantum Mechanical/Molecular Mechanical theoretical techniques and software tools to fundamental research in the areas of relevance to the development of pesticides, dyes, and to general solution photochemistry.

\section{Environmental and Molecular. Sciences Laboratory Construction Project}

Theory, Modeling, and Simulation, in collaboration with the Computing and Information Sciences Directorate, has been designing and developing the MSCF in the EMSL. Construction of the EMSL began in April 1994. The EMSL will give researchers the facilities and equipment they need to contribute to the knowledge base needed to solve complex environmental problems, from cleaning up toxic waste to preventing future contamination. As a national collaborative .research laboratory, the 200,000 square foot EMSL will house over 200 resident and visiting scientists from academia, industry, and other government laboratories. Construction was 
completed in the second half of 1996, and full operation of the facility is expected beginning fiscal year 1998.

Theory, Modeling, and Simulation has the responsibility to acquire the high performance computer hardware, and to develop and integrate the base set of molecular science software for large-scale computations in the EMSL. The software development efforts are described in detail elsewhere in this report. To support the development of advanced parallel software, a KSR2 parallel computer from Kendall Square Research was aquired in January 1994. The KSR2 system has 80 processors, each with 32 Mbytes of memory (totaling 2.5 Gbytes). The peak performance of the system is 80 MFLOPS per processor (6.4 GFLOPS total). PNNL benchmarks achieve up to half that rate. The system also has 48 Gbytes of disk and high speed FDDI and HiPPI interfaces to other EMSL development systems for databases, graphics, and distributed computing.

The procurement process began in late 1994 for the High Performance Computing System (HPCS-1) that will be housed in the EMSL facility. An evaluation panel composed of eight PNNL computational chemists and computer scientists drafted a specification containing the requirements for the massively parallel processor to be delivered in two phases (June 1996 and February 1997), After having sent the draft specification to forty-one interested firms, eleven firms participated in the presolicitation conference which was held in March 1995. The final solicitation was sent to over fifty (50) prospective offerors in June 1995, eight offerors requested benchmarking information, and four offerors responded by submitting proposals in September 1995. The four offerors were Convex (now a division of HP), Cray (now a Division of SGI), IBM, and SGI. We received three best and final offers from Cray, IBM, and SGI in December 1995.

In 1994-1995 the basic conceptual equipment design for the Graphics and Visualization Laboratory (GVL) was completed. The design included all computational, audio, and video components which will be located in the laboratory. Included in the design is the active link to the EMSL Seminar Room. A presenter in the Seminar Room will be able to video project a simulation running on the graphics servers. The design is based on technologies presently available. This design will be used as a basic functional standard and modified as required due to the expected technological advances. Based on this design we began the procurement process for the first of the two High Performance Graphics Servers.

The first meeting of the Molecular Science Computing Facility Advisory Committee was held in July.

\section{Northwest Lectureship in Physical Chemistry}

Dr. John C. Tully was presented the Northwest Lectureship in Physical Chemistry Award in 1994. The Lectureship, which is organized by the Environmental and Molecular Sciences Laboratory in cooperation with the University of Washington; Washington State University, University of Oregon, Oregon State University, and the University of Idaho, is periodically awarded to a physical chemist of the highest distinction. Dr. Tully, Head of the Materials Chemistry Research Department at AT\&T Bell Laboratories, presented a seminar on "Chemistry at Metal Surfaces: Molecular Dynamics with Electronic Transitions" in April 1994. 


\section{Honors, Awards, and Appointments}

Theory, Modeling, and Simulation

Bruce C. Garrett was promoted from a Scientist and Engineer, Level IV (S\&E IV) Chief Scientist, to an S\&E V Senior Chief Scientist.

Michel Dupuis joined the staff of the HPCC Group as an S\&E V Senior Chief Scientist.

Bruce C. Garrett was appointed Chair of the National Research Council's AFOSR High Energy Density Materials Panel.

Deborah S. Baker was promoted form a Level 5 Administrative Secretary, to a Level $6 \mathrm{Sr}$. Administrative Secretary.

Tony Hess was promoted from an S\&E III Sr. Research Scientist, to an S\&E IV Chief Scientist.

Rick A. Kendall was promoted from an S\&E III Sr. Research Scientist, to an S\&E IV Chief Scientist.

Jeffrey A. Nichols was appointed the High Performance Computational Chemistry Group Leader in 1995.

Rick L. Ornstein was appointed Graduate Faculty of the Department of Chemistry, Washington State University (WSU), Pullman, Washington. 1994-present.

Rick L. Ornstien was a Plenary Speaker \& Session Chair at the 4 th International Meeting of the Society for the Study of Xenobiotics, Seattle, Washington, August 1995.

Rick L. Ornstein was the Symposium Chair and a Session Co-chair at the 34th Hanford Symposium on Health and the Environmental, Pasco, Washington, October 1995.
Rick L. Ornstein was a Session Chair at the 3rd International Symposium on "In Situ and On-Site Bioremediation", San Diego, California, April 1995.

Rick L. Ornstein was a Session Chair at the 9th Symposium on Biomolecular Stereodynamics, S.U.N.Y., Albany, New York, June 1995.

Rick L. Ornstein was on the Program Committee and a Speaker at the 3rd International Symposium on Cytochrome P450 Diversity, Woods Hole, Massachussetts, October 1995.

Rick L. Ornstein was a nominee for 1995 Fitzner/Eberhardt Award at PNNL for Outstanding Contribution to Science \& Education.

Rick L. Ornstein was appointed as an Adjunct Professor of Microbiology, Molecular Biology and Biochemistry, University of Idaho, Moscow, Idaho. 1995-present.

Rick L. Ornstein was appointed as an Editoral Board Member (ad hoc): PROTEINS: Structure, Function \& Genetics. 1995-present

Rick L. Ornstein received the 1994-95 Associated Western Universities/Department of Energy Laboratory Distinguished Lecturer Award.

Erin N. Thornton was promoted from an S\&E II Research Scientist, to an S\&E III Sr. Research Scientist.

Rebecca R. Wattenburger was promoted from a Level 4 Sr. Secretary, to a Level 5 Administrative Secretary. 


\section{Visiting Scientists Program}

Theory, Modeling, and Simulation

During 1994 and 1995 a number of scientists visited the Pacific Northwest National Laboratory for collaborative work with staff in Theory, Modeling, and Simulation. These activities are listed below.

Dr. Susan C. Tucker, University of California Davis, Davis, California, April 20-24, 1994. With Molecular Theory Group.

Dr. Ken Jordan, University of Pittsburgh, Pittsburgh, Pennsylvania, May 8-11, 1994. With High Performance Computational Chemistry Group.

Dr. Brian T. Sutcliffe, University of York, York, England, May 18-20, 1994. With High Performance Computational Chemistry Group.

Professor Gregory A. Voth, Department of Chemistry, University of Pennsylvania, Philadelphia, Pennsylvania, June 26-July 2, 1994. With Molecular Theory and Modeling Group.

Dr. Daniel Edwards, University of Idaho, Moscow, Idaho, August 23, 1994. With Molecular Theory and Modeling Group.

Dr. Kenro Hashimoto, Tokyo Metropolitan University, Tokyo, Japan, September 16-20, 1994. With High Performance Computational Chemistry Group.

Dr. Klaus Schoeffel, Norsk Hydro Research Center, Porsgrunn, Norway, December 6-11, 1994. With High Performance Computational Chemistry Group.

Professor Subrata Ghose, University of Washington, Seattle, Washington, May 9-10, 1995. With Molecular Theory and Modeling Group.

Dr. Julian A. Peterson, University of Texas Southwest Medical Center, Dallas, Texas, May 17-20, 1995. With Biomolecular Modeling and Simulation Group.

Professor John Rehr, University of Washington, Seattle, Washington, May 17-18, 1995. With Molecular Theory and Modeling Group.

Dr. Jerry Boatz, US Air Force/Phillips Laboratory, Edwards AFB, California, June 19-23, 1995. With High Performance Computational Chemistry Group.

Dr. Stephen Rogers, US Air Force/Phillips Laboratory, Edwards AFB, California, June 20, 1995. With High Performance Computational Chemistry Group.

Dr. Paul W. Saxe, Biosym Technologies, Inc., San Diego, California, June 21, 1995. With Molecular Science Computing Facility.

Dr. Carl F. Melius, Sandia National Laboratories, Livermore, California, June 21, 1995. With Molecular Science Computing Facility.

Dr. Thomas Raeuchle, Cray Research, Inc., Eadan, Minnesota, June 21, 1995. With Molecular Science Computing Facility. 
Dr. Ray J. Meyers, Novell, Orem, Utah, June 21, 1995. With Molecular Science Computing Facility.

Dr. Cather Simpson, Sandia National Laboratories, Albuquerque, New Mexico, July 5-21, 1995. With High Performance Computational Chemistry Group.

Dr. Antonio Sgamelotti, University of Perugia, Perugia, Italy, July 31-August 1, 1995. With High Performance Computational Chemistry Group.

Professor Walter Thiel, University of Zurich, Zuerich, Switzerland, August 23-25, 1995. With High Performance Computational Chemistry Group.

Dr. Federico Nardi, European Molecular Biology Laboratory, Heidelberg, Germany, October 23November 4, 1995. With High Performance Computational Chemistry Group.

Dr. Joe T. Golab, Amoco Research Center, Naperville, Illinois, November 12-19, 1995 . With High Performance Computational Chemistry Group.

Dr. Christian Henn, SGI/Switzerland, December 13-14, 1995. With Extensible Computational Chemistry Environment Group.

Dr. Michael Teschner, SGI/Switzerland, December 13-14, 1995. With Extensible Computational Chemistry Environment Group.

Dr. Rebecca Wade, European Molecular Biology Laboratory, Heidelberg, Germany, December 18-20, 1995. With High Performance Computational Chemistry Group.

Dr. Susan C. Tucker, University of California Davis, Davis, California, December 26-30, 1995. With Molecular Theory and Modeling Group. 


\section{Seminar Program}

Theory, Modeling, and Simulation

During 1994 and 1995 Theory, Modeling, and Simulation hosted a number of invited seminar speakers. The speakers are listed below.

1994

Dr. Dimitris Agrafiotis, U.S. Geological Survey, May 6, “The Projected Electron Density Function: Theory and Applications"

Dr. Zhenqin Li, Biosym Technologies, Inc., January 7, "Molecular Modeling: From Force Fields to Structure \& Function"

Dr. Paul S. Bagus, IBM Alamaden Laboratory, January 17, "Electric Field and Vibrational Shifts of Chemisorbed Molecules"

Dr. Ya Jun Zheng, University of California, January 21, "Computer Simulation of Enzymatic Reaction Mechanism"

Dr. Shengbai Zhu, Alamaden Research Center, January 31, "Molecular Dynamics Simulations of Aqueous Solutions"

Dr. Ramona Taylor, Pennsylvania State University, February 1, "High Energy Particle Bombardment of Organic Films"

Dr. Toan BA Nguyen, Washington State University, February 4, "Theoretical Studies of Host-Guests Chemistry in Solution"

Professor Richard M. Stratt, Brown University, February 16, "How Big a Cluster Does it Take to Make a Liquid?"

Dr. Carlos Simmerling, University of Illinois at Chicago, February 18, "A Mean-Field Approach to Determination of Peptides Structures in Solution"

Professor Brett Jackson, University of Massachusetts, February 28, “Theoretical Models for Eley-Rideal Surface Reactions"

Dr. Arthur F. Voter, Los Alamos National Laboratory, March 8, "Atomistic Simulation of Surface Diffusion on Metals: Recent Progress and Future Challenges"

Dr. Arka Mukherjee, Princeton University, March 25, "Simulating the Low-Frequency Vibrations of Ni (II) Octaethylporphyrin: Expert System Design, Normal Mode Anaylis and Application to HemeProteins"

Dr. Venkatesan Renugopalakrichnan, Biosym Technologies, March 28, "Amelogenin: Structure and Dynamics of a 19 KD Protein" 
Dr. Richard P. Messmer, General Electric Corporate Research, March 29, "Accurate Valence-Bond Interpretable Wave Functions - A Starting Point for New Classical Force Fields"

Dr. John C. Tulley, AT\&T Bell Laboratories, April 4, "Chemistry at Metal Surfaces: Molecular Dynamics with Electronic Transitions"

Dr. William L. Jorgensen, Yale University, April 14, "Molecular Recognition in Organic and Biochemical Systems"

Dr. Yat-Ting Wong, National University of Singapore, April 20, "Density Functional Theory and LargeScale Quantum Simulations"

Dr. Susan C. Tucker , University of California, April 21, "Theoretical Models of Anisole Hydrolysis in Supercritical Water"

Dr. Leslie S. Perkins, Iowa State University, April 25, "Locomotion on FCC Surfaces: Diffusion via Hopping and Exchange"

Dr. Hugh Taylor, University of Texas, May 3 , "Geometry of the Formamide $n-\backslash p{ }^{\wedge}\left\{{ }^{*}\right\}$ Excited State by the Equations-of-Motion Couple-Cluster Method"

Dr. Monique Chacon-Taylor, University of Utah, May 3, "A Two-Dimension Model for Collisional Energy Transfer in Biomolecular Ion-Molecular Dynamics"

Dr. Tsun-Mei Chang, University of California-Los Angeles, May 6, "Non-Markovian Relaxation and Absorption Line Shape in Condensed Phases"

Dr. Ken Jordan, University of Pittsburgh, May 10, "Reactions of Hydrogen on the Si(100)-2x1 Surface"

Dr. Brian T. Sutcliffe, New York University, May 19, "The General Three Body Problem in Molecular Physics"

Dr. Paul Sherwood, Daresbury Laboratory, May 25, "Ab-Initio Calculations with Complex Environments: Approaches Based on Classical Electrostatics and Molecular Mechanics"

Professor James L. Skinner, University of Wisconsin, May 25, "Probing the Dynamics of Disordered Crystals: Spectral Diffusion of Individual Molecules"

Dr. Geoff Thornton, Manchester University, June 2, "XPS Studies of Ti02 Surfaces"

Dr. J. L. Musfeldt, Universite de Sherbrooke, June 28, "Microware Dielectric Studies of the Spin Density Wave State in (TMISF)2PF6"

Dr. Kurt K. Ding, University of Washington, July 8, "An Application of PRISM Calculations to Polymer Blends"

Dr. Kenneth Dyall, NASA Ames Research Center, July 11, "On the Choice of Zeroth-Order Hamiltonian for Second-Order Perturbation Theory with a CASSCF Reference Function"

Dr. Mark S. Gordon, Iowa State University, July 13, "Real Chemistry with Parallel Computers"

Dr. Qingsheng Zhao, Duke University, August 29, "Applications of Density-Functional Theory"

Dr. Nataraj Akkiraju, University of Illinois, September 9, "Visualizing Molecular Surfaces" 
Dr. Klaus Ruedenberg, Iowa State University, September 12, "Potential Energy Surfaces for Chemical Reactions"

Dr. Sunder Ramachandran, Colorado State University, September 12, "Towards an Understanding of Zeolite $Y$ as a Superacid Catalyst Using a PQEq"

Dr. Charles Martin, University of Chicago, September 23, "Ab Initio Basis of Semi-Empirical Pi-Electron Theory"

Professor Kenneth B. Eisenthal, Columbia University, September 29, "Laser Studies of Molecules at Liquid Interfaces"

Dr. Miles Deegan, University of Sussex, October 10, "Size-Extensive Electronic Structure Theories: Methodologies \& Applications"

Dr. Roger Loring, Cornell University, October 14, "Understanding Molecular Motions in Polymer Liquids"

Dr. Tjerk P Straatsma, University of Houston, October 17, "Free Energies from Molecular Simulations"

Dr. Tom George, Washington State University, October 18, "Theoretical Studies of Optical Intersubband Transitions in Semiconductor Heterostructure Quantum Wells"

Dr. Udo Becker, Virginia Polytechnic University, October 31, "The Calculation of STM Images as a Tool for Interpreting STM Data and Understanding Geochemical Surface Reactions"

Dr. V. Ara Apkarian, University of California - Irvine, November 2, "Breaking and Remaking of Bonds in Condensed Media in Real-Time"

Dr. Gregory K. Farber, Pensylvania State University, November 7, "Chymotrypsin"

Dr. Christian H. Bischof, Argonne National Laboratory, November 8, "Automated Differentiation of Computer Programs"

Dr. Jian Shen, Sterling Winthrope Pharmaceuticals, November 14, "Computer-Aided Molecular Design in Drug Discoveries"

Dr. Daruish H. Zadeh, State University of New York at Buffalo, December 3, "1. Heats of Formation By Non-local Density Functional Theory 2. Hydration of Mustard Gas in Ambient and Supercritical Water, a Combined Quantum Mechanics and Monte Carlo Approach"

Dr. Klaus Schoeffel, Norsk Hydro A/S Research Centre, December 9, "Theoretical Study on Chemisorption of NO in Copper-Exchanged Zeolites"

Dr. Guanhua Chen, University of Rochester, December 19, "Nonlinear Optical Properties of Conjugated Polyenes" 
Dr. Wei-Ping Hu, University of Minnesota, January 9, “Dual-Level Reaction-Path Dynamics: How do We Study Polyatomic Systems Accurately With Limited Computational Resources"

Dr. Stuart P. Slagle, University of Illinois, January 13, "Electrostatics of Protein-Protein Recognition"

Dr. William E. McCullen, Texas A\&M University, January 16, “Density Functionals for Polymers at Surfaces"

Dr. Alain Rochefort, Universite Laval, February 24, "Quantum Chemical DFT Study of the Interaction of Small Adsorbates with PD and Cu Cluster Methods"

Dr. Ilan Benjamin, University of California, February 27, "Charge Transfer Reactions at the Liquid/Liquid Interface"

Prof. Lorena S. Beese, Duke University, March 7, "Structure-Function Relations of DNA Polymerase Enzymes"

Dr. Gregory Petsko, Brandeis University, March 24, "Understanding and Exploiting the Glass Transition in Protein Dynamics"

Dr. Stephen K. Burley, The Rockefeller University, March 31, "Structure-function Relations for ProteinDNA Complexes"

Dr. William M. Atkins, University of Washington, March 27, “Novel Mechanisms of Cytochrome P450dependent Detoxification in Genetically Engineered Bacteria"

Dr. Michel Dupuis, IBM Corporation, April 12, "Large-Scale Calculations on the IBM SP2 for Electronic Structure and Reactivity"

Professor Branka Ladanyi, Colorado State University, April 27, "Instanteneous Normal Mode Analysis of Solvation Dynamics"

Dr. Yitian Xiao, Yale University, May 1, "Kinetics of Quantum Geochemical Processes: From Atmospheric to Water-Rock Interface"

Dr. J. D. Doll, Brown University, May 3, "The Interfacial Dynamics of Hydrogen in Metals"

Dr. Nathan J. Harris, Indiana University, June 2, "Accurate Computations of Harmonic Force Fields and Equilibrium Isotope Effects with Ab Initio MO Theory. Applications to Experimental Studies of Some Kinetic Isotope Effects"

Dr. Ignacio Luis Garzon-Sosa, Instituto De Fisica - UNAM, June 20, "Computer Simulation Studies of Metal Clusters and Non-Crystalline Seminconductors"

Professor Maciej Gutowski, University of Utah, July 10, "Multiply-Charged Anions and Anions with more than One Bound Electronic State"

Dr. Darrin York, Duke University, July 10, "Modeling Electrostatic Forces in Biological Molecules"

Dr. Theresa L. Windus, Northwestern University, July 13, "Object Oriented Programming in a Computational Chemistry Environment" 
Dr. Carlos A. Gonzalez, University of Pittsburgh, July 17, "Geometry Optimization of Large Scale Molecular Systems"

Dr. Roland Stumpf, Sandia National Laboratories, July 25, "H Enhanced Diffusion on Be(001)"

Dr. Diana Woodward, Southern Methodist University, July 28, "Modeling Complex Patterns Generated by Motile Bacteria"

Dr. George Fitzgerald, Cray Research, Inc., July 28, "UniChem: Architecture and Applications"

Dr. David Blackovich, Cray Research, Inc., August 9, "Cray Research and the Environmental Market"

Professor Walter Thiel, University of Zuerich, August 24, "New Developments in Semiempirical Methods"

Dr. Jichen Li, University of Salford, September 15,"Evidence for Two Kinds of Hydrogen Bonds in Ice"

Dr. Adrian M. Simper, British Nuclear Fuels, October 5, "British Nuclear Fuels: Who We are and the Research We Do"

Dr. Elizabeth Christopher, British Nuclear Fuels, October 5, “NMR Research in BNFL - Stray Field Imaging of Mortar"

Dr. Greg Voth, University of Pennsylvania, November 8, "Computer Simulations of Quantum Dynamical Processes in the Condensed Phase"

Dr. Jerry P. Greenberg, San Diego Supercomputing Center, November 9, "MDMovie and QMView: Programs for the Visual Analysis of Molecular Dynamics and Quantum Mechanical Calculations"

Dr. Andrew F.B. Thompson, Lawrence Livermore National Laboratory, November 20, "Stochastic Flow Simulation Using Parallel Computing"

Professor Kimihiko Hirao, The University of Tokyo, November 30, "Accurate Theory for Excited States"

Dr. Kurt Ding, University of Washington, December 11, "Polymer Chain Conformation and Molecular Constitution-Searching for Teflon Alternatives"

Dr. Rebecca Wade, European Molecular Biology Laboratory, December 19, "Protein-Ligand Interactions: From Simulation to Design" 


\section{Research Highlights}

Theory, Modeling, and Simulation

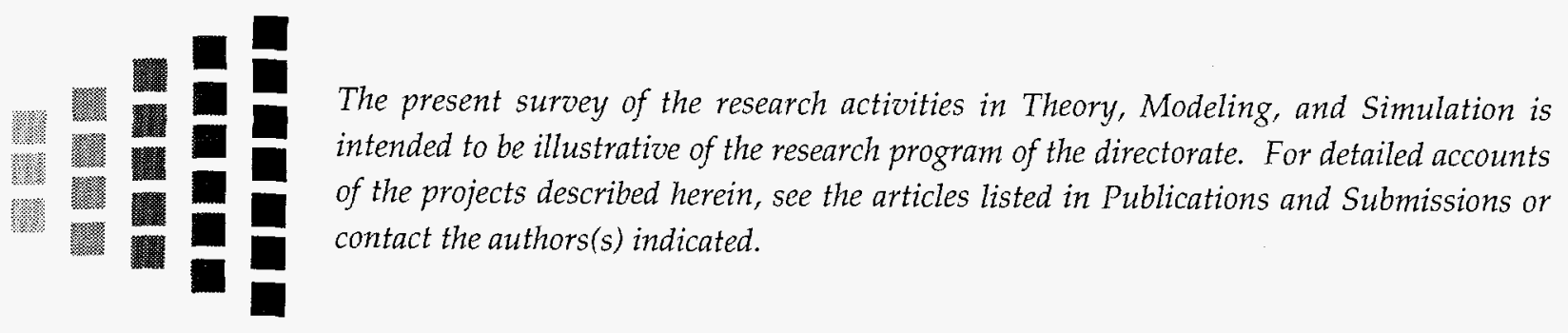

January 1, 1994 - December 31, 1995 


\section{Molecular Theory and Modeling}

Theory, Modeling, and Simulation

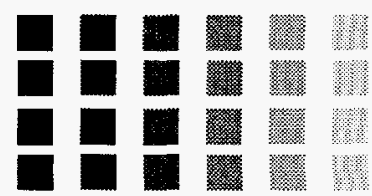

Research in the Molecular Theory and Modeling program focuses on increasing the understanding of molecular processes in condensed phases that are characteristic of natural and contaminated environments, as well as developing and testing new computational and theoretical methods. The research program integrates first principles studies of fundamental molecular processes in model systems with modeling of the complex molecular systems found in the environment. The objectives of the research are:

1. the application of available theoretical techniques to study fundamental molecularlevel processes

2. the extension of current state-of-the-art methods to treat progressively more complex systems

3. the use of molecular-level data to develop models of processes occurring in large complex systems

4. the integration of complementary theoretical approaches for examining processes occurring in the multi-species, multi-phase systems

5. linking theory and experiment through collaborative studies.

Applications in the program focus on four overlapping research areas: cluster chemistry, solution-phase chemistry, separations chemistry, and chemistry at interfaces. A common element in each of these areas is the need to understand molecular interactions in aqueous solutions or at environmentally important interfaces such as the interfaces between aqueous solutions and air, other liquids (e.g., organic liquids), minerals, and glasses. Development of new methods and technology is concentrated in the areas of electronic structure methods and reaction rate theory.

\section{Development of Electronic Structure Methods}

$A b$ initio electronic structure calculations are the basis for understanding the fundamental properties of molecules and their assemblages, including important information about interaction energies. The accuracy of electronic structure calculations is limited by the form of the many-electron expansion employed for the correlated wave function and by the one-electron basis set used to represent the molecular orbitals. The focus of the work in this area is the development and benchmarking of correlation consistent basis sets ${ }^{1-3}$ that systematically approach the complete basis set (CBS) limit and thereby allow currently available electronic structure methods to be adequately benchmarked.

Intrinsic Errors in $A b$ Initio Calculations: The Dissociation Energy of $\mathrm{N}_{2}$ (K. A. Peterson ${ }^{(a)}$ and T. H. Dunning, Jr.)

(a) Joint Appointment with Department of Chemistry, Washington State University

The importance of accurately treating electron correlation effects for describing molecular properties and processes is well known. The accuracy, however, of any correlated electronic structure calculation can be highly dependent on the oneparticle basis set used to represent the molecular orbitals. In particular, the interplay between the correlation method and basis set can lead to

The work reported in this section was supported by the Division of Chemical Sciences, Office of Basic Energy Sciences in the U.S. Department of Energy, by a grant of computer time at the National Energy Research Supercomputer Center by the Office of Scientific Computing in the U.S. Department of Energy, and by Laboratory Directed Research and Development funds. 
erratic results that limit our understanding of the true errors associated with the various correlation methods. Only by approaching the CBS limit can the intrinsic error of a theoretical method be assessed. Once the intrinsic error or inherent accuracy is determined, the merits of different correlated methods can be unambiguously compared.

Given the capability of accurately determining CBS limits for a given molecular property using the correlation consistent basis sets (cc-pVxZ, where $x=D, T, Q$, and 5$),{ }^{1-3}$ it is of great interest to compare the intrinsic errors of the correlation methods that are commonly used in electronic structure calculations. In our previous benchmark studies of diatomic hydrides and homonuclear diatomics containing first and second row atoms, ${ }^{4-7}$ the intrinsic errors of various configuration interaction (CI) methods were compared using the standard correlation consistent polarized valence basis sets. The $\mathrm{N}_{2}$ molecule was included in one of these CI studies. ${ }^{6}$ In the present work, the previous internally contracted multireference $\mathrm{CI}$ (CMRCI) results for the dissociation energy, $\mathrm{D}_{e}$, of $\mathrm{N}_{2}$ are compared to $\mathrm{D}_{e} \mathrm{~s}$ calculated by singles and doubles coupled cluster (CCSD); CCSD with a perturbative estimate of connected triple excitations [CCSD(T)]; and second-, third-, and fourth-order Møller-Plesset perturbation theory (MP2, MP3, MP4 ${ }^{\dagger}$ ). All of these latter methods are based on a Hartree-Fock single reference wave function and were selected because they are commonly used in a wide variety of computational chemistry applications. After extrapolating the series of $\mathrm{cc}-\mathrm{pVxZ}$ basis sets to the CBS limits, comparison to experiment yields the intrinsic errors in $\mathrm{D}_{e}$ for valence electron correlation calculations using each of these theoretical methods.

Calculated values of $\mathrm{D}_{e}$ for valence electron correlation are displayed graphically in Figure 1.1. For each method, smooth convergence with basis set size is observed, and the complete basis set limits are estimated by fitting the total energies to the simple exponential function

$$
\mathrm{D}_{e}(x)=\mathrm{D}_{e}(\infty)+\mathrm{Be}^{-\mathrm{C} x},
$$

+ Throughout this section MP4 refers to a full MP4 calculation, e.g. including singles, doubles, triples, and quadruples. where $x$ is the cardinal number of the basis set $(2$, 3,4 , and 5 for $\mathrm{DZ}, \mathrm{TZ}, \mathrm{QZ}$, and $5 \mathrm{Z}$ sets, respectively) and $\mathrm{D}_{e}(\infty)$ corresponds to the estimated CBS limit $(x \rightarrow \infty)$ for the dissociation energy. As observed in previous work, the CMRCI method yields an accurate value for $\mathrm{D}_{e}$ as one approaches the basis set limit. Comparing the CBS limit to the experimental value of $228.4 \mathrm{kcal} / \mathrm{mol}$ yields an intrinsic error for CMRCI of just $-0.8 \mathrm{kcal} / \mathrm{mol}$. Both the CMRCI+Q and CCSD(T) methods yield intrinsic errors that are somewhat larger, -1.6 $\mathrm{kcal} / \mathrm{mol}$.

While the dissociation energies calculated by multireference $C I$ and coupled cluster both smoothly converge to CBS limits below the experimental value, the CBS limit $\mathrm{D}_{e} s$ determined from the Moller-Plesset series exhibit an oscillatory behavior (see Figure 1.1). Compared to the experimental value, MP2 greatly overestimates $\mathrm{D}_{e}$, MP3 underestimates it, and $\mathrm{D}_{e}(\mathrm{MP} 4)$ is again too large but smaller than MP2. While the MP3 results are generally in poor agreement with experiment, certain sizes of basis sets lead to MP2 and MP4 dissociation energies close to the experimental value. In particular, at the MP2/cc-pVTZ level of theory, $\mathrm{D}_{e}$ differs from experiment by just $+0.8 \mathrm{kcal} / \mathrm{mol}$. Use of the cc-pVQZ basis set and $\mathrm{MP} 4$ results in an error of just $-0.4 \mathrm{kcal} / \mathrm{mol}$. However, in each case expansion of the basis set produces worse agreement with experiment. The observed good agreement is simply due to a fortuitous cancellation of errors and is not indicative of the inherent accuracy of the methods. Situations such as these severely limit our understanding of the correlation and basis set requirements of a particular property. At the estimated CBS limit, where the remaining errors are essentially due to the correlation method, both MP2 and MP4 overshoot the experimental value: MP2 is too large by $+11.6 \mathrm{kcal} / \mathrm{mol}$, while MP4 is in error by +3.4 $\mathrm{kcal} / \mathrm{mol}$.

The Accurate Calculation of Excited Electronic States: The Singlet-Triplet Splittings of $\mathrm{C}_{2}$, $\mathrm{CN}^{+}, \mathrm{BN}$, and $\mathrm{BO}^{+}$(K. A. Peterson ${ }^{(a)}$ )

(a) Joint Appointment with Department of Chemistry, Washington State University

The accurate calculation of excited electronic states of molecules can be an extremely challenging task for $a b$ initio electronic structure methods. 


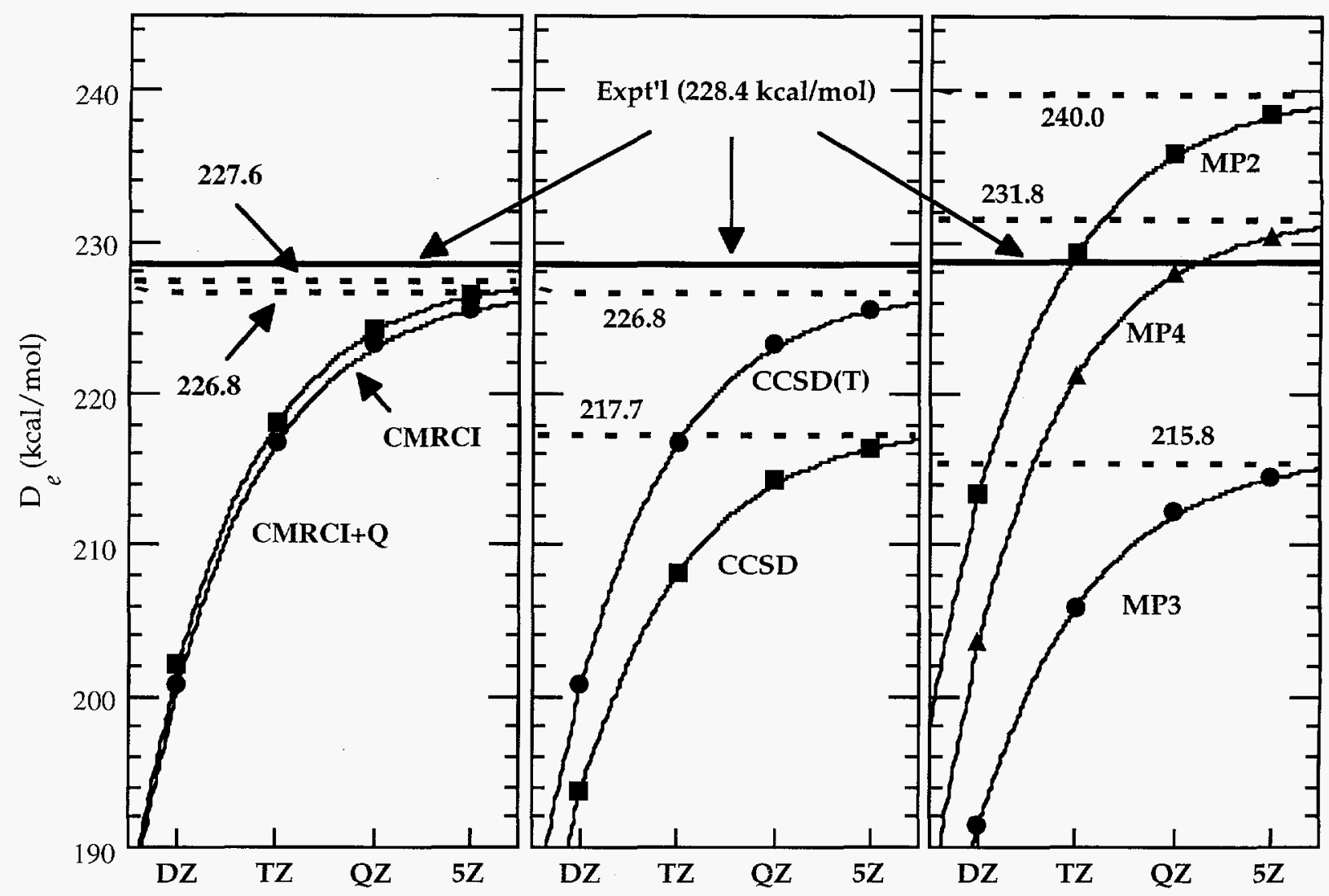

Figure 1.1. Calculated equilibrium dissociation energies for $\mathrm{N}_{2}$ as a function of the correlation consistent basis sets. The solid curves are fits to a simple exponential function, and the complete basis set limits for each method as estimated by these fits are indicated by the dotted lines. Experimental values are from Huber and Herzberg. ${ }^{8}$

In contrast to ground state wave functions, which are typically dominated by a single configuration state function (CSF), excited electronic states often exhibit strong multiconfiguration effects. Thus, a proper treatment of these systems requires use of a multireference method, such as multiconfiguration self-consistent field (MCSCF) or multireference configuration interaction (MRCI). The latter technique, which accounts for both nondynamical and dynamical correlation effects, has been shown to be extremely accurate, especially when full valence complete active space (CAS) reference functions are used. In addition to the n-particle basis or correlation treatment, the requirements of the one-particle basis set can also be severe for the accurate modeling of excited states. Particularly when energy differences, i.e., excitation energies, are being computed, basis sets which are well balanced with respect to different states are extremely important if accurate results are to be obtained.
Accurate determination of the ground electronic states of the 12-electron isoelectronic series, which includes $\mathrm{C}_{2}, \mathrm{BN} \mathrm{CN}^{+}$, and $\mathrm{BO}^{+}$, has historically been a difficult task. In each case the two lowest electronic states, ${ }^{1} \Sigma^{+}$and ${ }^{3} \Pi\left({ }^{1} \Sigma_{\mathrm{g}}^{+}\right.$and ${ }^{3} \Pi_{\mathcal{u}}$ for $C_{2}$ ), are separated by only a few tenths of an electron volt $(\mathrm{eV})$, and both theory and experiment have had difficulty in establishing which is the lowest state. Thus, the accurate calculation of the positions and properties of these states should provide a sensitive test of both the correlation method and one-particle basis set. Using highly correlated CMRCI wave functions, spectroscopic properties of $\mathrm{C}_{2}, \mathrm{CN}^{+}, \mathrm{BN}$, and $\mathrm{BO}^{+}$ have been calculated for their $1^{1} \Sigma^{+}$and $1^{3} \Pi$ electronic states. By utilizing the correlation consistent Gaussian basis sets of Dunning and coworkers, ${ }^{1-3}$ regular convergence of these properties with respect to increases in the one-particle basis set is observed. As in previous work, the convergence of total energy with respect to the 
cardinal number of the basis set is exponential, and from this an estimate of the value of the electronic excitation energy at the complete basis set limit can be obtained. Figure 1.2 shows the basis set dependence of the calculated electronic excitation energies for $\mathrm{C}_{2}, \mathrm{CN}^{+}, \mathrm{BN}$, and $\mathrm{BO}^{+}$. Both the standard cc-pVxZ basis sets and those augmented by extra diffuse functions, aug-ccpVxZ, are shown. Smooth convergence toward the CBS limit is observed in each case, and the difference between the $c c-p V x Z$ and aug-cc-pVxZ results are typically negligible for basis sets larger than cc-pVTZ.

By calibrating against the results for $\mathrm{C}_{2}$ and the ground state of $\mathrm{CN}^{+}$, accurate predictions of the spectroscopic constants of $\mathrm{CN}^{+}, \mathrm{BN}$, and $\mathrm{BO}^{+}$ were made. This was made possible by taking advantage of the regular convergence of the correlation consistent basis sets and the use of full valence complete active space self-consistent field (CASSCF) reference functions in the CMRCI
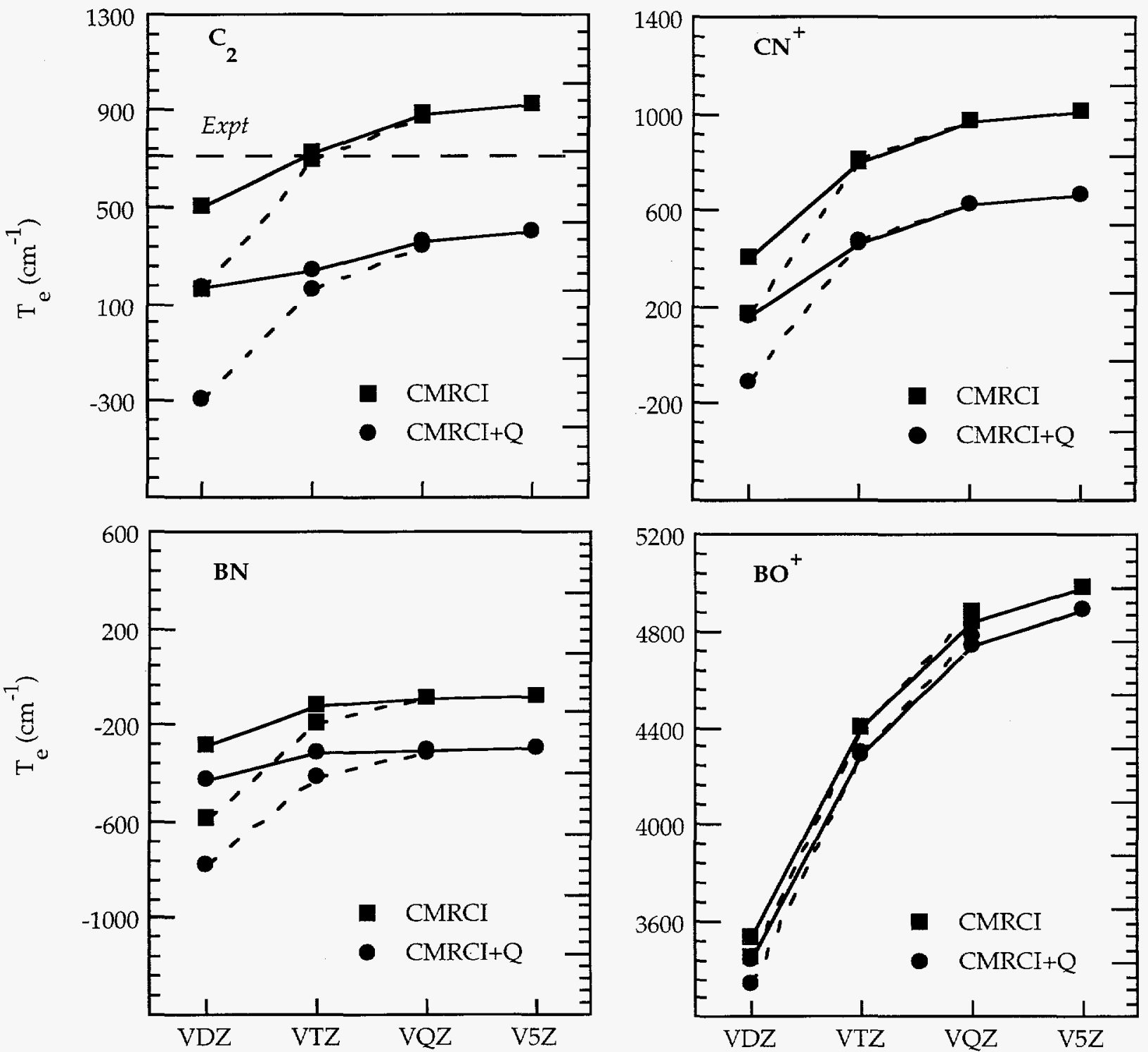

Figure 1.2. The dependence of the calculated singlet - triplet splittings $\left(\mathrm{T}_{e}\right)$ on the correlation consistent basis set. The solid lines indicate the standard cc-pVxZ basis sets, while the short dotted lines denote the aug-cc-pVxZ basis sets. Experimental results for $C_{2}$ are from Huber and Herzberg. ${ }^{8}$ 
calculations. Since the MRCI method had been previously shown to yield near-full CI quality results for the dissociation energy, $\mathrm{D}_{e}$, and the excitation energy $\mathrm{T}_{e}(a-X)$ of $\mathrm{C}_{2},{ }^{9}$ small corrections were applied to the CMRCI results that accurately reflected the difference between $\mathrm{CMRCI}$ and MRCI. The resulting predictions for $\mathrm{r}_{e}, \omega_{e}, \mathrm{D}_{e}$, and $\mathrm{T}_{e}$ arising from the current work after considering all the sources of error are summarized in Table 1.1 for each species. In agreement with previous studies, only the BN molecule has a ${ }^{3} \Pi$ ground electronic state, with a predicted splitting of just $190 \pm 100 \mathrm{~cm}^{-1}$. The $a^{3} \Pi$ state of $\mathrm{CN}^{+}$has a predicted excitation energy, $777 \pm 100 \mathrm{~cm}^{-1}$, that is similar in magnitude to $T_{e}$ of $\mathrm{C}_{2}$. The $\mathrm{BO}^{+}$ion, which has not been extensively studied experimentally, has an excited $a^{3} \Pi$ state that is predicted to lie much higher than the other members of this series.

Table 1.1. Predicted spectroscopic constants for $\mathrm{C}_{2}, \mathrm{CN}^{+}, \mathrm{BN}$, and $\mathrm{BO}^{+}$obtained from CMRCI calculations based on a full CASSCF reference function. Calculated results are for the estimated complete basis set limit.

\begin{tabular}{crrrr} 
Species & \multicolumn{1}{r}{$\begin{array}{c}\mathrm{r}_{e} \\
(\AA)\end{array}$} & $\begin{array}{c}\omega_{e} \\
\left(\mathrm{~cm}^{-1}\right)\end{array}$ & $\begin{array}{c}\mathrm{D}_{e} \\
(\mathrm{kcal} / \mathrm{mol})\end{array}$ & $\begin{array}{c}\mathrm{T}_{e} \\
\left(\mathrm{~cm}^{-1}\right)\end{array}$ \\
\hline $\mathrm{C}_{2}{ }^{1} \Sigma^{+}$ & & & & \\
$X^{1} \Sigma_{\mathrm{g}}$ & & & & \\
$a^{3} \Pi_{\mathrm{u}}$ & & & 147.1 & \\
$\mathrm{CN}^{+}$ & & & $\pm 1.5^{a}$ & \\
$\mathrm{X}^{1} \Sigma^{+}$ & & & & $778^{b}$ \\
& & & 175.3 & \\
$a^{3} \Pi$ & 1.241 & 1711 & 117.1 & 880 \\
& \pm 0.002 & \pm 15 & \pm 1.5 & \pm 100 \\
$\mathrm{BN}^{3}$ & & & & \\
$\mathrm{X}^{3} \Pi$ & 1.325 & 1526 & 106.0 & \\
& \pm 0.002 & \pm 15 & \pm 1.5 & \\
$a^{1} \Sigma^{+}$ & 1.275 & 1705 & 161.2 & 190 \\
& \pm 0.002 & \pm 15 & \pm 1.5 & \pm 100 \\
$\mathrm{BO}^{+}$ & & & & \\
$X^{1} \Sigma^{+}$ & 1.203 & 1822 & 131.7 & \\
& \pm 0.002 & \pm 15 & \pm 1.5 & \\
$a^{3} \Pi$ & 1.302 & 1484 & 72.1 & 5000 \\
& \pm 0.002 & \pm 15 & \pm 1.5 & \pm 200
\end{tabular}

a The experimental value is $147.8 \pm 0.5 \mathrm{kcal} / \mathrm{mol}^{10}$

$b$ The experimental value is $716 \mathrm{~cm}^{-1} .8$
Accurate $A b$ Initio Force Fields and Vibrational Spectra: The Dipole Moment Function of HCN (K. A. Peterson ${ }^{(a)}$ )

(a) Joint Appointment with Department of Chemistry, Washington State University

Three-dimensional, near-equilibrium potential energy and dipole moment surfaces have been calculated for the linear triatomics $\mathrm{HCN}, \mathrm{HNC}$, $\mathrm{HCO}^{+}, \mathrm{HOC}^{+}$, and $\mathrm{HNN}^{+}$using the series of correlation consistent basis sets from $c c-p V D Z$ to $c c-$ pVQZ. Both internally contracted multireference $\mathrm{CI}(\mathrm{CMRCI})$ and $\mathrm{CCSD}(\mathrm{T})$ potential energy functions were computed, and the dipole moments were calculated from the $\mathrm{CMRCI}$ wave functions. Using analytical fits of the cc-pVQZ $\operatorname{CCSD}(\mathrm{T})$ potential energy and CMRCI dipole moment values, three-dimensional variational calculations were carried out to determine rotationalvibrational energy levels and infrared intensities. In the variational calculations, both a finite basis representation (FBR) and a discrete variable representation (DVR) have been investigated for the bend coordinate. Not surprisingly, the DVR method was found to give the fastest convergence for the higher-lying vibrational energy levels. In both cases, the radial coordinates were represented by basis sets constructed of Morse oscillators, while the bend coordinate utilized a basis set based on Legendre polynomials.

Table 1.2 compares calculated and experimental integrated infrared band intensities for HCN. In

Table 1.2. CMRCI and experimental integrated band intensities $(\mathrm{km} / \mathrm{mol})$ for $\mathrm{HCN}$.

\begin{tabular}{ccl} 
Band $^{a}$ & Theory & Exp. ${ }^{11}$ \\
\hline 001 & 0.003 & $\sim 0.03$ \\
010 & 70.4 & $57.7(11)$ \\
100 & 53.2 & $53.3(3)$ \\
101 & 0.26 & $0.27(3)$ \\
011 & 0.70 & $0.71(7)$ \\
111 & 0.0018 & $0.0010(1)$ \\
020 & 8.0 & $8.5(2)$ \\
200 & 1.28 & $0.85(1)$ \\
300 & 0.0296 & $0.0255(1)$
\end{tabular}

$a v_{1}=\mathrm{CH}$ stretch, $v_{2}=\mathrm{HCN}$ bend, $v_{3}=\mathrm{CN}$ stretch. 
general, infrared intensities are difficult to accurately determine experimentally, and the results shown in Table 1.2 indicate that state-of-the-art $a b$ initio calculations can be extremely valuable in characterizing ro-vibrational spectra. The largest deviations from experimental values are observed for the $v_{1}$ (CN stretch) and $v_{2}$ (bend) fundamentals, where the experimental uncertainties are the largest.

A $b$ Initio Studies of Magnetic Hyperfine and Electrostatic Properties of Small Molecules (D. F. Feller, ${ }^{(a)}$ E. D. Glendening, ${ }^{(b)}$ K. A. Peterson, ${ }^{(c)}$ R. J. Miller, ${ }^{(d)}$ and E. A. McCullough, Jr. $\left.{ }^{(e)}\right)$

(a) Joint Appointment with the Computational Chemistry Environment Group

(b) AWU Postdoctoral Research Associate

(c) Joint Appointment with Department of Chemistry, Washington State University

(d) Materials and Chemical Sciences Center, PNNL

(e) Department of Chemistry, Utah State University

Magnetic hyperfine interactions shed light on the distribution of unpaired spin density in molecules with one or more open shells. Because they place stringent requirements on the overall quality of the wave function and the geometry, they are a demanding testbed for high quality $a b$ initio methods. Equally demanding is the prediction of electrostatic properties (e.g., the dipole moment) in molecules possessing diffuse charge densities.

As a follow-up to our recent study of the magnetic hyperfine structure parameters for the $X^{2} \Pi$ state of NO, we examined its low-lying A ${ }^{2} \Sigma^{+}$ Rydberg state. A variety of $a b$ initio methods, including MRCI and coupled cluster methods were applied in an attempt to reproduce the ${ }^{14} \mathrm{~N}$ and ${ }^{17} \mathrm{O}$ isotropic hyperfine interactions and the recently reported dipole moment. For the former properties, the full CI limit was approached through a systematic sequence of ever-moreextensive, CI wave functions that would, in principle, include the full $\mathrm{CI}$ as its final element. Our best $C I$ value for the magnetic hyperfine constant $\mathrm{A}_{\mathrm{iso}}$ on ${ }^{14} \mathrm{~N}$ (39 MHz) compares favorably with the $41 \mathrm{MHz}$ found experimentally. The dipole moment was found to be extremely sensitive to both the choice of orbitals (i.e., canonical restricted Hartree-Fock verses CASSCF verses natural orbitals) and to the technique used to evaluate the property (i.e., expectation value verses finite field). Values which are nearly $50 \%$ higher or lower than experiment were found using theoretical methods which would normally predict values within $10 \%$ for most ground states.

\section{Benchmarking Electronic Structure Software and Hardware (D. F. Feller ${ }^{(a)}$ and R. A. Kendall $\left.^{(b)}\right)$}

(a) Joint Appointment with the Computational Chemistry Environment Group

(b) High Performance Computational Chemistry Group

Hardware and software technologies continue to change at a rapid pace. It is often difficult for EMSL scientists to obtain reliable information dealing with the relative speeds of various programs and their implementation on various computers, specifically which are the most effective for a given type of calculation. To help answer that question, at least within the narrow context of $a b$ initio electronic structure methods, we undertook an extensive series of calculations with $10 a b$ initio packages running on 28 different workstation and traditional supercomputer platforms. The types of calculations span the range from Hartree-Fock to high-level correlated methods. In the current study we extended the list of methods to include some preliminary findings on density functional methods and parallel computing. The results of this benchmark investigation are available in hard copy or electronic formats (both in PostScript or WWW-compatible forms). ${ }^{\dagger}$ Representative speedups for a variety of molecules for the GAMESS program running in parallel are shown in Figure 1.3.

\section{Development of Reaction Rate Theories}

Chemical reactions play an important role in environmental systems (e.g., they can affect the transport of contaminants as well as their transformation, and they are responsible for the degradation of organic species in waste tanks).

+ A copy of the PostScript version can be obtained by typing ftp://pnlg.pnl.gov/qcbenchmarks/ from a web browser or by anonymous ftp to pnlg.pnl.gov in directory qcbenchmark. 
KSR2/GAMESS(US) Direct Hartree-Fock

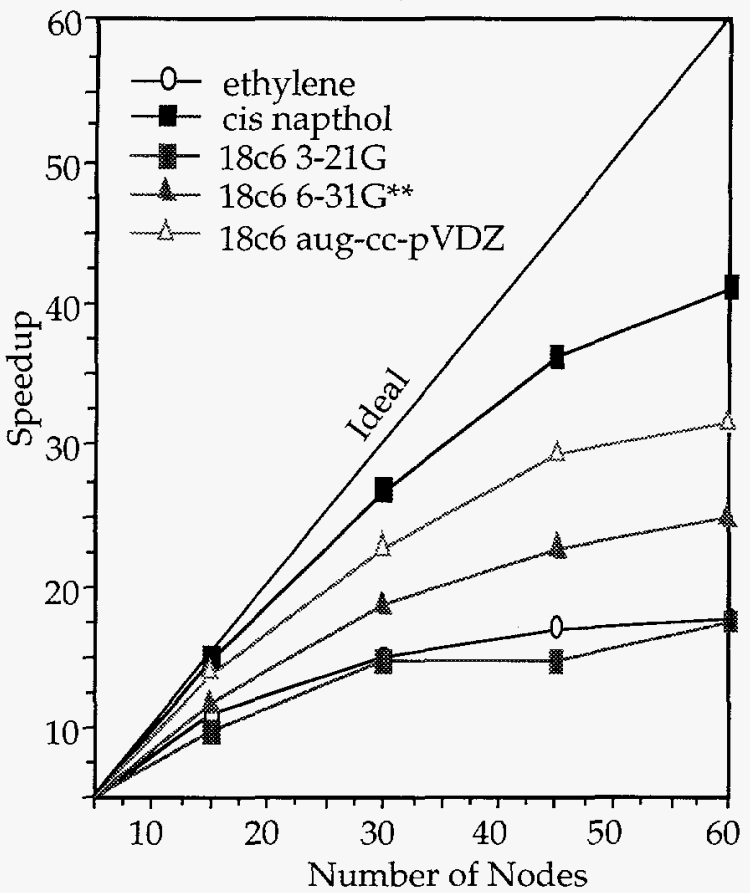

Figure 1.3. Speedup Curve for GAMESS(US) Running on the KSR/2.

Reliable fate and transport as well as process models for these complex systems require knowledge of the reaction rates. The challenge is to develop rate theories for chemical reactions occurring in condensed media, primarily aqueous solutions. A further complication in these systems is that the reactions occur in acidic or alkaline solutions where proton transfer processes are important. Accurate treatment of the dynamics of these light particles requires the use of quantum mechanical methods.

Methods for predicting reliable rate constants for gas-phase reactions have been developed over the last twenty years. The most successful and widely used methods are those based upon transition state theory (TST). ${ }^{12}$ In particular, variational transition state theory (VTST) with multidimensional semiclassical corrections for quantum mechanical tunneling provides the most practical method for treating polyatomic reactions. ${ }^{13-17}$ More recently, another approach, which includes quantum mechanical effects in a TST framework, is the centroid-density path integral method. ${ }^{18-21}$ The focus of the current work is the extension of the VTST method to solution-phase reactions and the development of the centroid density method to include important variational effects.

\section{Variational Transition State Theory for Activated Chemical Reactions in Solution (B. C. Garrett and G. K. Schenter)}

A strategy is outlined for applying quantized variational transition state theory with multidimensional semiclassical tunneling methods to activated chemical reactions in solution. ${ }^{22}$ The approach taken here separates the system into a cluster model that is treated explicitly and the 'solvent' that is treated approximately. The approach includes both equilibrium solvation effects on interaction energies and nonequilibrium. effects that enter through a solvent friction model.

Our approach is to define an effective solvation potential, equivalent to a multidimensional potential of mean force, for the solute (or cluster) degrees of freedom using (classical) statistical perturbation theory. (In some cases, such as when masses of either the solute of solvent are sufficiently light, a quantum mechanical evaluation of the effective potential may be necessary. In principle this can be accomplished using path integral methods. ${ }^{23,24}$ ) Evaluation of the quantized VTST rate constant proceeds as in the gasphase calculations, but using the effective solvation potential thereby including the effects of equilibrium solvation. Variational transition state theory calculations do not require a knowledge of the global potential energy surface; only the potential in a quadratic region around the minimum energy path (MEP) is needed. The first and second derivative information along the MEP of the equilibrium solvation potential can be obtained directly from ensemble averages over the solvent coordinates. The potential along the MEP can be obtained from the first derivative information by line integration.

The equilibrium solvation model includes the average effect of the solvent on the solute energetics but neglects any dynamical influence of the solvent. At the next level of complexity, it is possible to introduce dynamical effects by considering fluctuations in the force on the solute coordinates about the mean force. To estimate the 
fluctuations, a set of effective harmonic oscillator coordinates are introduced. It is assumed that the dynamics of the solute are sufficiently described by the equations of motion derived from an effective nonequilibrium solvation Hamiltonian in which the harmonic oscillators are linearly coupled to the solute coordinates. The classical dynamics for this nonequilibrium solvation model are equivalent to a Generalized Langevin Equation of motion in the limit of a continuum of ascillators. The parameters of the oscillator model are obtained from the timedependent force-force correlation function that can be obtained from molecular dynamics calculations. Quantized VTST calculations again proceed as for gas-phase reactions but using the nonequilibrium solvation Hamiltonian model.

Tunneling in the Presence of a Bath: A Generalized Transition State Theory Approach (D. G. Truhlar, ${ }^{(a)}$ Y.-P. Liu, ${ }^{(b)}$ G. K. Schenter, and B. C. Garrett)

(a) Department of Chemistry, University of Minnesota
(b) Chemistry Department, Brookhaven National Laboratory

The treatment of overbarrier dynamics in VTST involves the free-energy-of-activation profile and this can readily be extended to solution-phase reactions by using the potential of mean force along the reaction coordinate. However, the most successful approaches to including tunneling in a practical way in the gas-phase case have involved calculating the transmission coefficient for tunneling through the ground-state adiabatic potential curve (the ground-state approximation), and this cannot be generalized quite so straightforwardly. The ground-state approximation for thermal transmission coefficients in the gas phase is most readily justified by the reasonable assumption that at low temperatures (where tunneling is most important) most reactive flux passes through the transition state region in the ground-state energy level or with at most a few low-frequency modes excited so that the effective potential may still be reasonably approximated by a shifted version of the ground-state adiabatic potential. In solution though, the density of states is high, and the ground state is physically unattainable without freezing. In this work we present a more robust argument for defining an effective potential for tunneling in terms of quan- tities defined by statistical mechanics or statistical thermodynamics. ${ }^{25}$

Within the adiabatic approximation, the correction factor for quantum motion along the reaction coordinate is defined as the ratio of the thermal average of the quantum transmission probabilities and the thermal average of the classical transmission probabilities. The classical probabilities are functions of the total energy $E$ and the maximum in the adiabatic potential curve (zero for energies below the barrier and one above). The quantum mechanical probabilities are not only functions of the total energy but are functionals of the adiabatic potential curves (both the height and the shape of the barriers). In the ground-state approximation, the shapes of all adiabatic barriers are approximated by the shape of the ground-state potential curve. For tunneling in systems with a bath of low frequency oscillator, rather than use the shape of a single adiabatic state (such as the ground state), we use an average, or mean-shape, potential that better characterizes tunneling through the states that are thermally populated. To define the mean-shape potential, the tunneling probability is expanded through first order variations with respect to a reference potential. The mean-shape potential is equal to the reference potential for which the first order terms in the thermally averaged transmission coefficient vanish. The resulting approximate transmission coefficient is appropriate for use with microcanonical variational theory, and a canonical mean-shape potential is similarly defined to give a transmission coefficient that is appropriate for use with canonical variational theory. The canonical mean-shape potential is approximately equal to the potential of mean force along the reaction coordinate.

Nonequilibrium Solvation for an AqueousPhase Reaction: Kinetic Isotope Effects for the Addition of Hydrogen to Benzene (B. C. Garrett and G. K. Schenter)

Variational transition state theory with semiclassical tunneling corrections is applied to a model of $\mathrm{H}$ atom addition to benzene in the gas phase and aqueous solution. ${ }^{26}$ The model allows the separation of equilibrium (static) solvation effects on the free energy of activation from nonequilibrium (dynamic) solvation effects that enter 
through frictional terms. For this model and within a classical mechanical treatment, the static effect of the solvent on the equilibrium free energy of solvation is independent of the mass of the solute. Therefore, within a classical equilibrium solvation model, kinetic isotope effects are the same for gas and aqueous phases. Observations of changes of kinetic isotope effects (KIEs) upon solvation can therefore indicate when dynamic solvent effects are important.

In a recent paper, Roduner and Bartels ${ }^{27}$ have compared aqueous-phase experimental rate constants for the addition of $\mathrm{H}^{27} \mathrm{D}, 27$ and $\mathrm{Mu}^{28}$ to benzene with the gas-phase rate constants for $\mathrm{H}^{29} \mathrm{D},{ }^{29}$ and $\mathrm{Mu}^{28}$. The rate constant for the reaction in water is faster than the rate constant in the gas phase by factors between 20 and 40 for the $\mathrm{H}$ and $\mathrm{D}$ isotopes. Roduner and Bartels have shown that equilibrium solvation effects can account for this effect - the transition state complex is more stable in solvent than the separately solvated reactants. The transition state has a less positive free energy of solvation than reactants, effectively lowering the activation free energy in aqueous solution relative to the gas-phase value. However, they find that equilibrium solvation cannot account for the much smaller increase in the rate constant for $\mathrm{Mu}$ (about a factor of 3) and propose "that there may be a more fundamental, dynamic reason for the occurrence of such a pronounced mass effect." In this work, we provide a theoretical study of the effects of nonequilibrium (dynamical) aqueous solvation on KIEs for $\mathrm{H}$ addition to benzene.

We employ a simplified model of the reaction that incorporates the features necessary to reproduce the rate constant dependence on temperature, isotope mass, and environment (i.e., gas and aqueous phases). The dynamical influence of the solvent is treated by a harmonic bath linearly coupled to the solute reaction coordinate. Variational transition state theory with semiclassical tunneling corrections is used to study the qualitative trends in the addition reaction of $\mathrm{H}$ to benzene.

The model calculations show that the nonequilibrium solvation effects are small for the $\mathrm{H}$ and $\mathrm{D}$ addition reactions and that an equilibrium solvation model can adequately reproduce the experimental ratio of the aqueous and gas phase rate constants for these isotopes (i.e., the computed values are within $10-12 \%$ of the experimental ones). However, the equilibrium solvation model overestimates this ratio for the $\mathrm{Mu}$ isotope by over a factor 30 . The calculations with the nonequilibrium solvation model were carried out for a range of values of the solvent friction. To estimate the range, we use the Stokes-Einstein relationship with the diffusion constant for $\mathrm{H}$ in water yielding values of the strength of the friction $\eta_{0}$ less than 20 atomic units. The plot of the ratio of the calculated nonequilibrium and equilibrium rate constants (Figure 1.4) clearly shows a strong dependence of the rate constant on nonequilibrium solvation effects for the $\mathrm{Mu}$ isotope and a weak dependence for $H$ and $D$. Choosing a value of $\eta_{0}=6 \mathrm{au}$, we find that we are able to reproduce the experimental trends for all three isotopes. The dramatic suppression of the $\mathrm{Mu}$ rate when going from an equilibrium solvation description to a nonequilibrium description is required to reproduce the experimental trend.

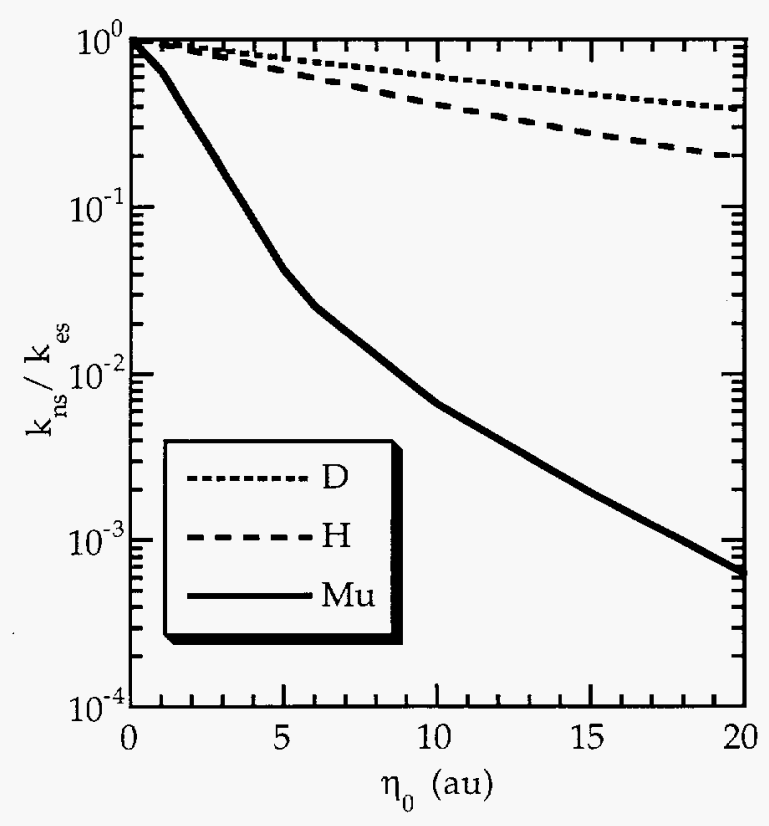

Figure 1.4. Variation of the ratio of nonequilibrium and equilibrium VTST rate constants with the magnitude of the solvent friction. 


\section{Accurate Calculation of Quantum Mechanical Reaction Rates at Low Temperatures (G. K. Schenter, M. Messina, ${ }^{(a)}$ and B. C. Garrett)}

(a) Department of Chemistry, University of California, San Diego

Recently we have spent much effort studying centroid density rate theories ${ }^{18-21}$ as they provide a tractable procedure for calculating reaction rate constants in multidimensional systems. In the current work, we investigate the low temperature behavior and the identification of an appropriate "dynamical bottleneck" in this regime. Within a transition state theory framework, the identification of the dynamical bottleneck is equivalent to the specification of a multidimensional dividing surface separating reactants from products. The most convenient bottle neck is that determined from classical dynamics. For a one-dimensional problem, the classical bottleneck coincides with the maximum of the potential, even for an asymmetric potential. In the study described here we found that this is not the case when we consider the deep tunneling regime.

The low temperature behavior of the reactive rate constant was investigated for tunneling through a one-dimensional asymmetric Eckart barrier and results are present in Table 1.3. In this case, the use of the classical location of the bottleneck at the top of the potential results in transmission coefficients that are too large by as much as five orders of magnitude. By using a variational procedure whereby the best transmission coefficient is found by locating the position of the centroid to find the minimum value, we recover good agreement with exact results. This method successfully interpolates between the low and high temperature limits and can be readily generalized to multidimensional systems.

Reversible Work Transition State Theory and Its Application to the Dissociative Adsorption of Hydrogen (G. K. Schenter, G. Mills, ${ }^{(a)}$ and H. Jonsson $\left.^{(a)}\right)$

(a) Department of Chemistry, University of Washington

A practical method of finding free energy barriers for reactive processes in high-dimensional quantum mechanical systems has been developed
Table 1.3. Exact quantum and approximate centroid density correction factors for tunneling of a particle with the mass of hydrogen through an asymmetric Eckart potential with intrinsic barrier height of $5.7 \mathrm{kcal} / \mathrm{mol}$, (imaginary) barrier frequency of $1045 \mathrm{~cm}^{-1}$, and exoergicity of -4.4 $\mathrm{kcal} / \mathrm{mol}$. The centroid density correction factor $\Gamma_{\mathrm{CD}}$ places the dividing surface at the barrier maximum and the variational centroid density correction factor $\Gamma_{V C D}$ locates the dividing surface to minimize the rate constant.

$\begin{array}{llll}\mathrm{T}(\mathrm{K}) & \Gamma_{\text {Exact }} & \Gamma_{\mathrm{CD}} & \Gamma_{\mathrm{VCD}} \\ 250 & 9.0 & 8.6 & 8.0 \\ 200 & 3.7(1)^{\mathrm{a}} & 4.0(1) & 3.3(1) \\ 150 & 9.0(2) & 1.8(3) & 8.9(2) \\ 125 & 1.9(4) & 7.7(4) & 2.1(4) \\ 100 & 2.8(6) & 4.9(7) & 3.9(6) \\ 75 & 1.9(10) & 5.8(12) & 5.6(10) \\ 50 & 1.9(18) & 4.4(23) & 4.5(19)\end{array}$

a Numbers in parentheses are powers of ten.

recently and was used to calculate the dissociative sticking probability of $\mathrm{H}_{2}$ on a metal surface. $^{30}$ The method ${ }^{31}$ consists of merging the concepts based on the evaluation of the reversible work of a process with recent developments of centroid density based quantum transition state theory. ${ }^{18,19,21,31-35}$ Centroid density methods have been applied to various test problems and have given reliable estimates for rate constants for systems where quantum zero-point and tunneling effects are significant. In the methods, quantum statistical averages in terms of Feynman path integration are evaluated using Monte Carlo techniques. No explicit quantum dynamics is involved, allowing the methods to be tractable for multidimensional systems. In addition, the inclusion of harmonic heat baths and the coupling of classical degrees of freedom is straightforward. The reversible work involved in moving a multidimensional hyperplane from a reactant region to a saddle point, considering both its translation and rotation, is calculated to provide an estimate of the rate constant.

The system studied consisted of thermalized hydrogen gas on a $\mathrm{Cu}(110)$ surface, corresponding 
to the experimental measurements of Campbell and coworkers. ${ }^{36,37}$ For this system it is necessary to consider the quantum mechanical nature of the hydrogen motion due to its light mass. In this case both tunneling and zero-point effects are significant. In the calculation, two hydrogen atoms and eight surface $\mathrm{Cu}$ atoms were included quantum mechanically with more than two hundred atoms in the $\mathrm{Cu}$ crystal included classically. An embedded-atom form for the $\mathrm{H}_{2}$ and $\mathrm{H}-\mathrm{Cu}$ interaction potential was chosen and fitted to data for the $\mathrm{H}_{2}$ dimer and chemisorbed hydrogen. The potential barrier for the reaction was scaled to be consistent with existing ab initio calculations. Thermal averages were carried out over both molecular and surface degrees of freedom. Effective activation energies for adsorption and desorption were determined as a function of temperature and were found to be significantly lowered by tunneling at low temperature (see Figure 1.5). For the model potential that was considered, the system crossed over from classical to quantum behavior at about $400 \mathrm{~K}$ for $\mathrm{H}_{2}$ and $300 \mathrm{~K}$ for $\mathrm{D}_{2}$. In the quantum mechanical regime, the activation energy is significantly reduced, the free energy barriers are found to be flattened as a function of configuration, and delo-

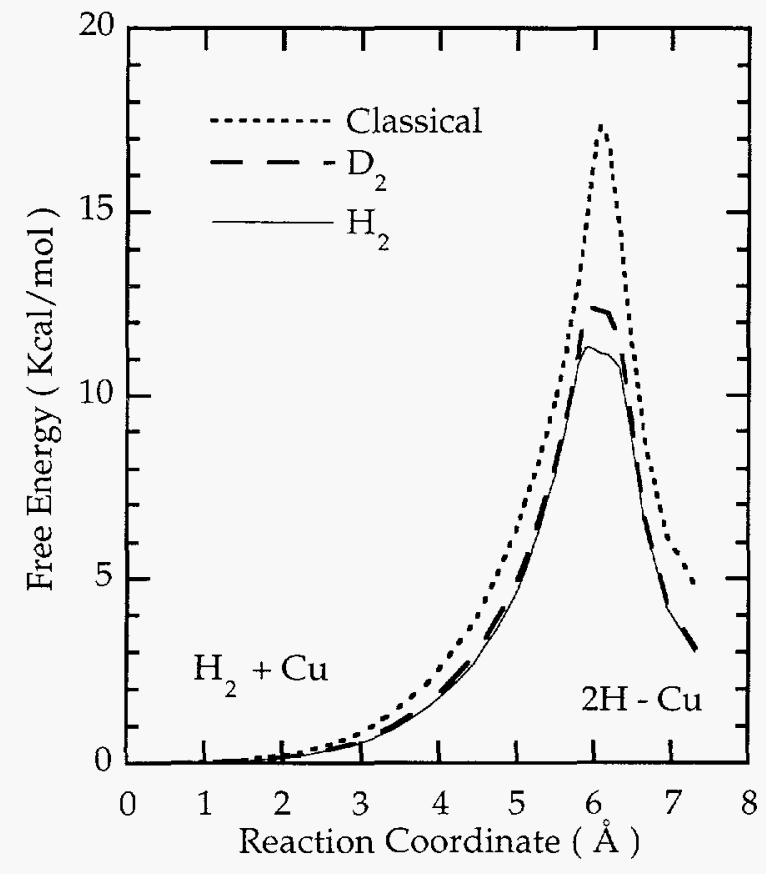

Figure 1.5. The reversible work free energy as a function of the location of the dividing surface along the MEP at $100 \mathrm{~K}$. calization is significantly greater at the barrier than in the reactant state, indicating that tunneling becomes the dominant transition mechanism. The resulting Arrhenius plots for the rate of $\mathrm{H}_{2}$ and $\mathrm{D}_{2}$ adsorption and desorption versus temperature were found to be consistent with available experimental measurements.

The Influence of Solvent on the Rate of Chemical Reactions in the Energy-Diffusion Regime (G. K. Schenter, S. K. Reese, ${ }^{(a)}$ and S. C. Tucker $^{(a)}$ )

(a) Department of Chemistry, University of California, Davis

Chemical reactions typically require passage over an energy barrier of activation, often limiting progress so that a reaction becomes a rare event. Sufficient energy must be concentrated in reactive motion for the event to occur. Often the necessary energy is supplied from a "thermal bath"made up of solvent molecules. In the case when the transfer of energy from the solvent to the solute reactive motion is sufficiently inefficient and slow compared to the time it takes to geometrically form a reaction complex, the energy transfer process will become rate limiting and the chemical reaction will be "energy diffusion" controlled, or the process will be in an "energy diffusion" regime. Some examples of processes where such energy transfer control has been observed include photo-induced geminate recombination of $\mathrm{I}_{2}$ in a variety of liquid solvents and unimolecular dissociation in high pressure gases.

In a recent study, ${ }^{38}$ the role that the solvent spectral profile plays in determining the rate of an energy-diffusion process was investigated. The solvent spectral profile, or the spectral density as probed by the solute, is a measure of both the strength of coupling of the solute reactive motion to the solvent and the time scale for which the coupling occurs. It was found that for the case when the time scale of the solvent response is sufficiently long, the resulting rate constant for the reaction can depend quite dramatically on the specific functional form of the friction kernel that characterizes the solvent response.

In this study we constructed a model system consisting of a one-dimensional dissociative solute reaction coordinate coupled to a solvent bath 
with dynamics described using a Generalized Langevin Equation (GLE). Two forms for the friction kernel in the GLE were compared, one having an exponential time dependence and the other having a Gaussian time dependence. In comparing the two, the integrated viscosity, a measure of the strength of coupling between the solute and the bath, and the characteristic time scale of the kernel were set equal to one another. When comparing the total overall rate constant for the two cases, a difference of over seventeen orders of magnitude was observed. This was due to the inefficiency of vibrational energy relaxation for the Gaussian kernel relative to the exponential kernel. Numerical solution of the GLE using a reactive flux formulation provided estimates for the rate of the process associated with relaxation off the barrier of the reaction. To estimate the rate associated with the process of gaining energy to be in the vicinity of the barrier top, a mean first passage time estimate was made.

In this study we transformed the GLE to a Hamiltonian form, allowing us to explicitly follow the energy gain of specific solvent collective modes and to determine which modes are important for each process. This also allowed us to test methods based on transition state theory. We found that the solute energy relaxation may be thought of as a two-step process, barrier energy relaxation (BER) and vibrational energy relaxation (VER) into the solvent well. Each of these processes were controlled by different regions of the solvent's spectral profile. BER was governed by the low frequency solvent modes, while VER was governed by the higher frequency solvent modes. These results provided guidelines for the use of a GLE to model solution phase reaction dynamics. If it is not possible to determine the friction kernel, either empirically or from simulation, a reasonable form must be chosen, depending on the magnitude and one characteristic time scale. If the BER rate is of interest, the results will not be critically dependent of the exact functional form chosen. However, if VER may be slow enough to play a role in the reactive dynamics, the overall rate will be sensitive to the high frequency region of the solvent's spectral profile and a more detailed description of the form of the friction kernel is required. Furthermore, for the case of a Gaussian friction kernel, methods based on the evaluation of reactive flux and transition state theory did not predict the correct overall rate. In contrast, for the exponential friction kernel case both reactive flux calculations and transition state theory calculations gave accurate estimates for the rate constant.

\section{Modeling Molecular Processes in Clusters}

Research in cluster chemistry is focused on the structure and properties of aqueous clusters and on the energetics and dynamics of molecular processes involving such clusters. Molecular clusters offer a unique opportunity to examine the transition from the gaseous phase to condensed phases and to enhance our understanding of the intermolecular interactions between solutes and water. In addition, to the extent that clusters model the solution phase, studies of molecular clusters provide an opportunity to ascertain the effects of solvation on chemical reactions: comparison of the pathways, energetics, and dynamics of the "solvated" reaction with the gas-phase reaction will lead to insights into the detailed role that the solvent plays in altering the mechanism and rates of reactions in solution.

The methodologies developed to model gasphase molecules and molecular processes are being applied to the study of the aqueous clusters. However, solution of the electronic and nuclear Schrödinger equation for aqueous clusters poses two challenges. First, the number of atoms involved in the calculations increases with the size of the cluster and accurate calculations rapidly become intractable. Second, the resulting potential energy surface possesses multiple minima that complicates the characterization and representation of the potential surface and subsequent dynamical studies. The primary focus of work in this area is the understanding of the structure and energetics of aqueous clusters and the development of improved interaction models for these systems.

Variation of the Three-Body Interaction Energy Term in Different Water Trimer Configurations (S. S. Xantheas)

We computed the magnitudes of the two- and three-body energy terms and their contribution 
to the interaction energy for different water trimer arrangements, shown in Figure 1.6. The calculations were performed at the second through fourth order many-body perturbation (MP2 and MP4) levels of theory using the augmented correlation-consistent polarized valence basis set of double zeta quality..$^{1-3}$ We considered the configurations in which the water molecules act as proton donor-acceptors $(d a)$, double acceptors $(a a)$ and double donors $(d d)$, respectively. The global minimum corresponds to a "cyclic" structure of $C_{1}$ symmetry in which all water molecules act as proton donors-acceptors $(d a)$ having one hydrogen bond per water molecule. ${ }^{39,40}$ We have optimized the geometry of the $(d d, a, a)$ configuration keeping $C_{2 v}$ symmetry. The internal coordinates of the double donor molecule are $0.969 \AA$ and $105.3^{\circ}$, whereas the ones of the single acceptors are 0.966

Water trimer configurations

(da,da,da)

2

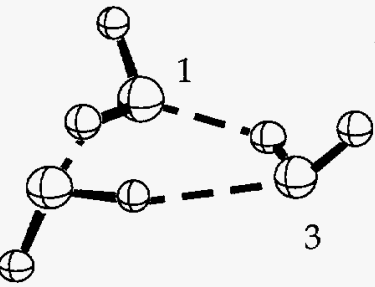

$(\mathrm{dd}, \mathrm{a}, \mathrm{a})$

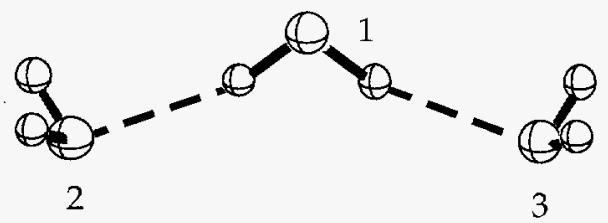

(aa,dd,da)

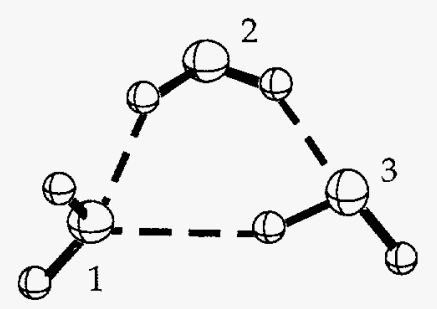

Figure 1.6. Geometries for the three water trimer minima considered in this study with labeling of the individual fragments: ( $\mathrm{da}, \mathrm{da}, \mathrm{da}$ ) each water is both a proton donor and a proton acceptor; (dd,a,a) double donor, acceptor, acceptor; (aa,dd,da) double acceptor, double donor, donor acceptor. and $104.1^{\circ}$, respectively. The $\mathrm{O}-\mathrm{O}$ separation is $2.945 \AA$ and the hydrogen bond angle $\delta(\mathrm{O} \cdots \mathrm{H}-\mathrm{O})$ $=163.8^{\circ}$. A vibrational analysis at the $\mathrm{C}_{2 \mathrm{v}}$ optimal geometry confirms that it is a minimum. We shall hereafter refer to it as the "open" minimum. It lies $4.2 \mathrm{kcal} / \mathrm{mol}$ above the "cyclic" minimum and the cyclic minimum is stable by $13.9 \mathrm{kcal} / \mathrm{mol}$. We have not been able to obtain a minimum energy structure of the $(a a, d d, d a)$ configuration denoted as structure " 10 " by Mó and Yáñez. ${ }^{41}$ We have found that this structure collapses to the global "cyclic" minimum. We have, therefore, chosen the geometry reported by Mó and Yáñez as representative of the $(a a, d d, d a)$ arrangement to perform the decomposition of the interaction energy.

We used the scheme of Hankins, Moskowitz, and Stillinger ${ }^{42}$ to perform the decomposition of the interaction energy. Basis set superposition error (BSSE) corrections were estimated by the function counterpoise method ${ }^{43}$ as outlined in a previous study. ${ }^{44}$ The results are shown in Table 1.4 for the three different trimer configurations of Figure 1.6. The energy difference between the "cyclic" and "open" minima has its origin in the difference between both the two- $(\sim 3.4 \mathrm{kcal} / \mathrm{mol})$ and threebody $(\sim 3 \mathrm{kcal} / \mathrm{mol})$ terms. The $(a a, d d, d a)$ structure has all three types of different water arrangements: a double acceptor 1), a double donor 2 ), and a donor-acceptor 3). The relaxation energy term is largely due to the fact that the fragment internal coordinates reported by Mó and Yáñez ${ }^{41}$ at the MP2/6-311+G(d,p) level of theory were used. The two-body interactions

Table 1.4. Decomposition of MP2 interaction energies (with BSSE correction) for the various water trimer configurations.

\begin{tabular}{|c|c|c|c|c|c|}
\hline \multicolumn{3}{|c|}{ Terms } & \multicolumn{3}{|c|}{ Interaction energies ( $\mathrm{kcal}, \mathrm{mol})$} \\
\hline$i$ & $j$ & $k$ & $(d a, d a, d a)$ & $(d d, a, a)$ & $(a a, d d, d a)$ \\
\hline 1 & 2 & & -4.07 & -4.45 & -3.66 \\
\hline 1 & 3 & & -3.70 & -4.45 & -3.22 \\
\hline 2 & 3 & & -4.04 & 0.53 & -1.39 \\
\hline 1 & 2 & 3 & -2.45 & 0.46 & 0.22 \\
\hline \multicolumn{3}{|c|}{ Relaxation } & 0.38 & 0.07 & 1.93 \\
\hline \multicolumn{3}{|c|}{ Total 2-body } & -11.80 & -8.37 & -8.27 \\
\hline \multicolumn{3}{|c|}{ 3-body } & -2.45 & 0.46 & 0.22 \\
\hline
\end{tabular}


(1-2) and (1-3) are large since they better resemble a water dimer interaction than the smaller (2-3) interaction. The three-body term is also repulsive for this arrangement, which lies $8.4 \mathrm{kcal} / \mathrm{mol}$ higher in energy than the global minimum. As regards the relative contributions of the various terms to the interaction energy, the three-body term is large and attractive $(\sim 18 \%)$ for the "cyclic" but smaller and repulsive for both the "open" ( $\sim \%)$ and $(a a, d d, d a)(\sim 4 \%)$ configurations, respectively. The MP2 and MP4 results were found to differ only slightly $(<2 \%)$.

Our results confirm the hypothesis of White and Davidson ${ }^{45}$ that donor-acceptor configurations lead to enhanced interactions, therefore producing clusters of increased stability. Water trimer configurations in which the water molecules act as either double donors, double acceptors, or mixed donor-acceptors have different three-body contributions which, for the structures considered here, were repulsive. It, therefore, is reasonable to conclude that the three-body contribution to the interaction energy for water trimers is important as it can change in sign and magnitude according to the local hydrogen bond arrangement and should, therefore, be included in any realistic potential modeling of these systems.

\section{Comparison of Density Functional with MP2 for Cyclic Water Clusters (S. S. Xantheas)}

The optimal structures, harmonic vibrational frequencies, and association energies of ring water clusters $\left(\mathrm{H}_{2} \mathrm{O}\right)_{n}, n=1-6$ were computed using density functional theory (DFT). Benchmark calculations on the water monomer and dimer were performed using the exchange functionals of Slater (S) and Becke (B), the correlation functionals of Lee-Yang-Parr (LYP), Vosko-Wilk-Nusair (VWN), and Perdew - local (PL) and gradientcorrected (P86) - as well as their combinations as implemented in the Gaussian-92/DFT (revision F.3) suite of programs. ${ }^{46}$ We used the augmented correlation-consistent polarized valence orbital basis set of double (aug-cc-pVDZ) zeta quality in comparisons of the DFT with earlier MP2 results. ${ }^{39,40}$ Better overall agreement with the MP2 results seems to be achieved by using the exchange-correlation combinations, although their relative differences are subtle. All combinations yield better results than HartreeFock for the structural and electric properties of water. The corresponding results for the structure and binding energy of the water dimer are shown in Table 1.5. For a definition of the internal coordinates reported in this table see earlier studies. ${ }^{39}$ Better overall agreement with the MP2 and experimental results for the water monomer and dimer was observed for the B-LYP and B-P86 functional combinations. We have chosen to use the B-LYP functional for the study of the larger $(n \geq 3)$ clusters since it produces a more accurate $\mathrm{O}-\mathrm{O}$ separation and a dipole moment for the water dimer than the B-P86 functional (cf. Table 1.5).

The structural trends in the inter- and intramolecular internal coordinates with cluster size have been studied with the B-LYP functional and compared with previous MP2 results. 39,40 The variation of the nearest neighbor $\mathrm{O}-\mathrm{O}$ separation with cluster size is plotted in Figure 1.7(a). We note a systematic contraction of the nearest-neighbor $\mathrm{O}-\mathrm{O}$ separation with cluster size, similar to the one previously observed at the Hartree-Fock and MP2 levels of theory. ${ }^{39}$ The solid (MP2) and broken (B-LYP) lines in Figure 1.7(a) correspond to a fit of the exponential

$$
\mathrm{R}_{e}^{(O-O)}(n)=\mathrm{A}+\mathrm{Be}^{\mathrm{Cn}},
$$

where $\mathrm{n}$ is the size of the cluster. The HartreeFock results (dotted line) are also included for comparison. For the trimer and pentamer, the average of the corresponding $\mathrm{O}-\mathrm{O}$ separations was used. The optimal O-O separations with the B-LYP functional follow closely the ones at the MP2 level for $n=2-4$. Furthermore, their variation with cluster size in the $2 \leq n \leq 6$ domain is exponential (the correlation coefficient for the fit is 0.9993 ). The hydrogen bond angle $\mathrm{O} \cdots \mathrm{H}-\mathrm{O}$ was also observed to become more linear with increasing cluster size, resulting in an almost linear hydrogen bond for the hexamer. The results with the B-LYP functional are close (within $<1^{\circ}$ ) to the corresponding MP2 results for the $n=2-4$ clusters. The same level of agreement between B-LYP and MP2 was also observed for the variation of the intramolecular water internal coordinates with cluster size.

In general, B-LYP underestimates, with respect to MP2, both intramolecular bending and stretching frequencies by $50 \mathrm{~cm}^{-1}$ and $150-270 \mathrm{~cm}^{-1}$, respectively. It reproduces the blue shift in the bending frequencies although by a somewhat lesser 
Table 1.5. Optimal geometries, dipole moments, and binding energies for the water dimer with the various exchange-correlation functional combinations. All calculations were performed with the aug-ccpVDZ set and the "fine" grid option for the numerical integration. The internal coordinates $\phi_{\mathrm{d}}$ and $\chi_{\mathrm{a}}$ are zero due to the $\mathrm{C}_{\mathrm{s}}$ plane of symmetry. (Number in parenthesis includes BSSE correction.)

\begin{tabular}{|c|c|c|c|c|c|c|}
\hline Method & $\begin{array}{c}R_{e}(\mathrm{O}-\mathrm{O}) \\
(\AA) \\
\end{array}$ & $\begin{array}{r}\theta_{\mathrm{a}} \\
\left({ }^{\circ}\right) \\
\end{array}$ & $\begin{array}{l}\theta_{\mathrm{d}} \\
\left({ }^{\circ}\right) \\
\end{array}$ & $\begin{array}{l}\chi_{\mathrm{d}} \\
\left(^{\circ}\right) \\
\end{array}$ & $\begin{array}{c}\mu \\
\text { (Debye) } \\
\end{array}$ & $\begin{array}{c}D_{e} \\
(\mathrm{kcal} / \mathrm{mol}) \\
\end{array}$ \\
\hline $\mathrm{S}$ & 2.790 & 67.4 & -59.6 & 7.1 & 2.57 & -7.7 \\
\hline B & 3.254 & 56.0 & -57.3 & 5.4 & 2.46 & -2.10 \\
\hline S-PL & 2.719 & 67.3 & -59.5 & 6.8 & 2.62 & -9.0 \\
\hline S-P86 & 2.631 & 77.0 & -62.0 & 9.3 & 2.38 & -12.2 \\
\hline S-VWN & 2.710 & 67.3 & -59.5 & 6.8 & 2.62 & -9.2 \\
\hline S-LYP & 2.663 & 70.1 & -60.8 & 7.9 & 2.58 & -11.6 \\
\hline B-PL & 3.092 & 57.5 & -57.4 & 5.3 & 2.50 & -2.8 \\
\hline B-P86 & 2.894 & 64.9 & -58.0 & 5.9 & 2.48 & -4.4 \\
\hline B-VWN & 3.070 & 58.1 & -57.5 & 5.3 & 2.50 & -2.9 \\
\hline B-LYP & 2.939 & 60.2 & -58.0 & 5.7 & 2.56 & $-4.3(-4.1)$ \\
\hline Hartree-Fock & 3.032 & 46.3 & -56.3 & 3.3 & 2.91 & $-3.9(-3.7)$ \\
\hline $\mathrm{MP} 2$ & 2.911 & 57.3 & -57.9 & 5.7 & 2.73 & $-5.3(-4.4)$ \\
\hline Exp. & $2.946^{a}$ & $57 \pm 10^{a}$ & $-51 \pm 10^{a}$ & $<30^{a}$ & $2.60^{b}$ & $\begin{array}{l}-5.4 \pm 0.7^{c} \\
-5.4 \pm 0.2^{d}\end{array}$ \\
\hline $\begin{array}{ll}a & \text { Reference } \\
b & \text { Reference } \\
& 48 \\
c & \text { Reference } \\
d 9 \\
d & \text { See reference }\end{array}$ & & & & & & \\
\hline
\end{tabular}

amount with respect to the MP2 results. The shifts for the "bridge" OH stretches, $\Delta \omega(\mathrm{O}-\mathrm{H})_{b}$, with respect to the average $\mathrm{OH}$ stretches of the monomer with cluster size are shown in Figure 1.7(b). The open circles denote the results with the B-LYP functional, whereas the filled squares correspond to the MP2 results. The lines trace the average shifts with cluster size at the B-LYP (broken line), MP2 (solid line), and Hartree-Fock (dotted line) levels of theory. B-LYP seems to overestimate $\Delta \omega(\mathrm{O}-\mathrm{H})_{\mathrm{b}}$ by $\sim 100 \mathrm{~cm}^{-1}$ with respect to MP2. It exhibits the same variation pattern observed at the Hartree-Fock level (i.e., levels off after $n=4$ to a value of $\sim 500 \mathrm{~cm}^{-1}$ ).

Finally, the agreement between B-LYP and MP2 for the total and incremental association energies is satisfactory with the aug-cc-pVDZ basis set, especially when BSSE corrections are included.
Investigation of Acetyl Chloride Photodissociation by Photofragment Imaging (S. Deshmukh, ${ }^{(a)}$ J. D. Myers, ${ }^{(b)}$ S. S. Xantheas, and W. P. Hess ${ }^{(c)}$ )

(a) AWU Postdoctoral Research Associate, Chemical Structure and Dynamics Program, EMSL

(b) Computing and Information Science Program, EMSL

(c) Chemical Structure and Dynamics Program, EMSL

Theoretical calculations were performed to assist in the interpretation of experimental results obtained by an ion imaging technique during photodissociation of acetyl chloride at $236 \mathrm{~nm} .{ }^{51}$ The recorded $\mathrm{Cl}$ images display anisotropic angular distributions, characteristic of a prompt, impulsive dissociation of the $\mathrm{C}-\mathrm{Cl}$ bond. Furthermore, the experimental data suggest that a fraction (about $28 \%$ ) of the $\mathrm{CH}_{3} \mathrm{CO}$, produced as a primary photoproduct, subsequently decomposes to form $\mathrm{CH}_{3}$ and $\mathrm{CO}$. The optimal structures of the 

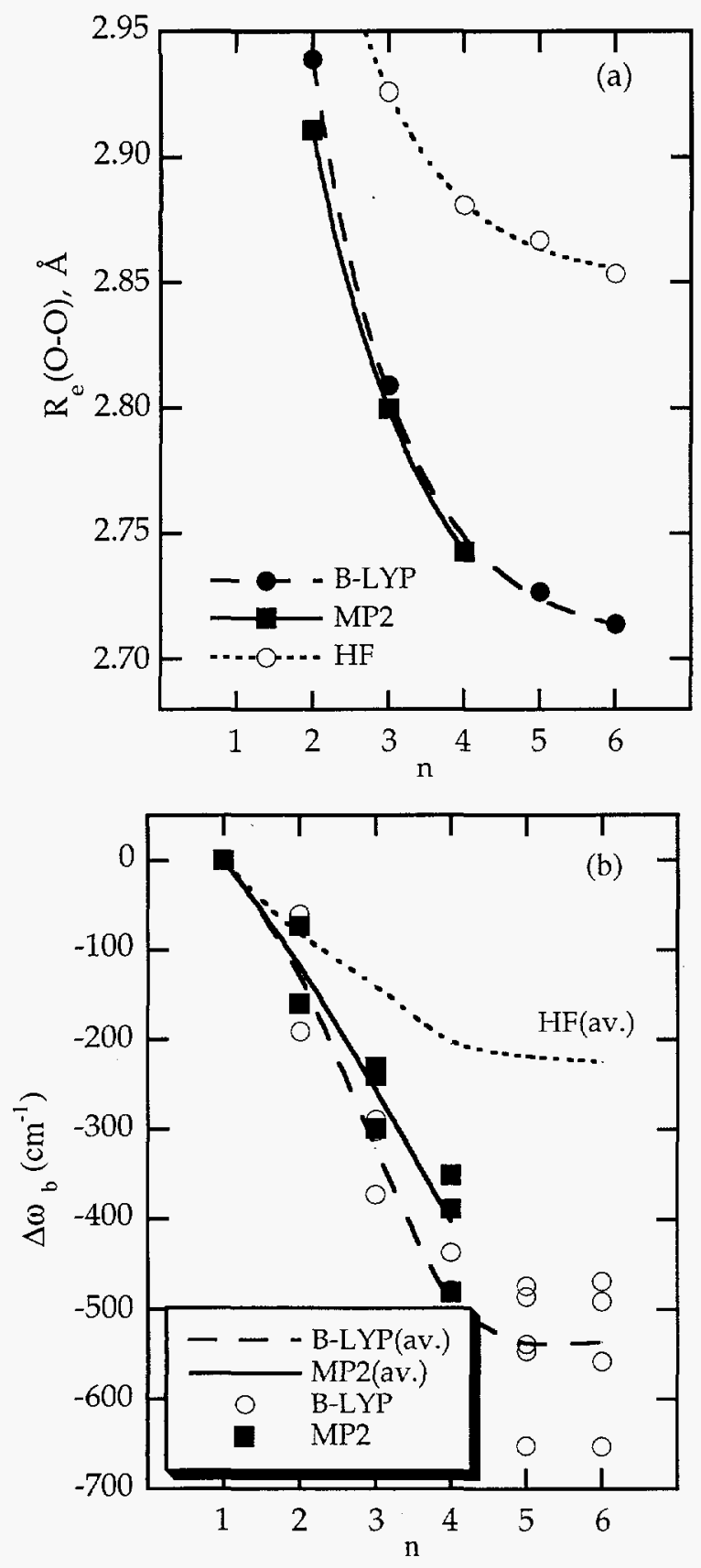

Figure 1.7. Variation of (a) the nearest-neighbor $\mathrm{O}-\mathrm{O}$ separations and (b) "bridge" $\mathrm{OH}$ frequency shifts of $\left(\mathrm{H}_{2} \mathrm{O}\right)_{n}$ with cluster size at the B-LYP (broken line), MP2 (solid line), and Hartree-Fock (dotted line) levels of theory.

minimum $\left({ }^{2} \mathrm{~A}^{\prime}\right)$ and transition state corresponding to the dissociation of the acetyl radical $\left(\mathrm{CH}_{3} \mathrm{CO}\right)$ and the optimal geometry of $\mathrm{CH}_{3} \mathrm{COCl}$ $\left({ }^{1} \mathrm{~A}^{\prime}\right)$ are shown in Figure 1.8(a) at the MP2/ccpVTZ level of theory. The energy profile for the
$\mathrm{CH}_{3} \mathrm{CO}$ dissociation along the $\mathrm{C}-\mathrm{C}$ reaction coordinate at the MP2/cc-pVTZ level of theory is shown in Figure 1.8(b). The values of the (C-C-O) and $(\mathrm{H}-\mathrm{C}-\mathrm{H})$ angles are indicated for values along the $\mathrm{C}-\mathrm{C}$ reaction coordinate. The barrier for $\mathrm{CH}_{3} \mathrm{CO}$ dissociation was experimentally estimated at $16.2 \pm 2 \mathrm{kcal} / \mathrm{mol}$ and theoretically predicted at $19.1 \mathrm{kcal} / \mathrm{mol}$ at the MP2 $/ \mathrm{cc}-\mathrm{pVTZ}$ level of theory. The dissociation proceeds toward a nearly $\mathrm{C}_{\mathrm{s}}$ pathway (i.e., the atoms $\mathrm{O}, \mathrm{C}, \mathrm{C}$, and $\mathrm{H}_{a}$ remain nearly on the same plane). The geometry of the transition state for the acetyl radical dissociation is a more open structure with a relaxed, nearly planar $\mathrm{CH}_{3}$ group, and a contracted (C-C$\mathrm{O})$ bond angle. As the $\mathrm{C}-\mathrm{C}$ bond lengthens, the pyramidal methyl group slowly relaxes to near the planar structure of free methyl. The gently relaxing $(\mathrm{H}-\mathrm{C}-\mathrm{H})$ angle allows the methyl radical to form without exciting the $v_{2}$ bending vibration, and since the methyl tripod does not cock off axis during dissociation, little rotation of the methyl radicals is produced. The $\mathrm{CO}$ product displays no observable vibrational excitation, however significant $\mathrm{CO}$ rotational excitation is observed. This is consistent with the calculated $\mathrm{C}=\mathrm{O}$ bond contraction to near that of the free $\mathrm{CO}$ along the reaction path. It appears that these motions occur gently, allowing the methyl and $\mathrm{CO}$ fragments to transform adiabatically into ground vibrational state products. The rotational state distribution is also qualitatively explained by examining the differences in the ground state acetyl chloride and transition state acetyl structures.

Structures and Energetics of $\mathrm{F}^{-}\left(\mathrm{H}_{2} \mathrm{O}\right)_{n}, \mathrm{n}=1-3$ Clusters (S. S. Xantheas and T. H. Dunning, Jr.)

We have computed the optimal structures and harmonic vibrational frequencies of the $\mathrm{F}^{-}\left(\mathrm{H}_{2} \mathrm{O}\right)_{n}$, $n=1-3$ clusters at the MP2 level of theory. ${ }^{52}$ For the $n=2$ and 3 cases, the minimum energy configurations correspond to asymmetric structures where the ion sits outside the water cluster. The optimal structures of the clusters are shown in Figure 1.9. The $n=1$ minimum has $\mathrm{C}_{\mathrm{s}}$ symmetry, whereas the $n=2$ minimum exhibits no symmetry elements. For the $n=3$ case we have found two minima. One has $C_{3}$ symmetry with all three water molecules acting both as proton donors (to the ion and to another water) and proton acceptors (from another water). This $\mathrm{C}_{3}$ structure resembles a "pyramidal" structure with the ion at the top and the three water molecules at the base 


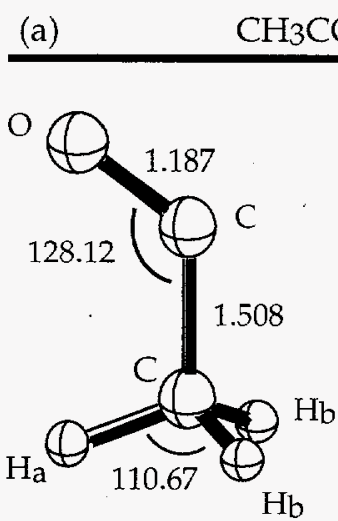

Minimum

\section{(2A' state)}
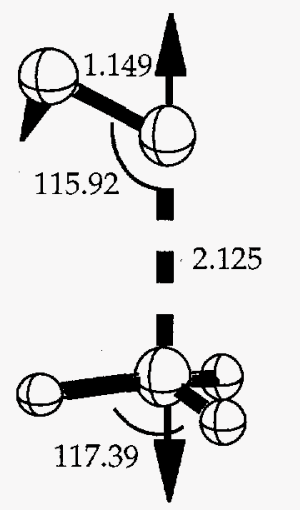

Transition State

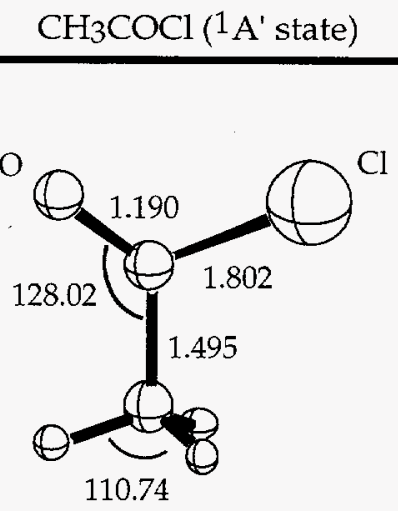

110.74

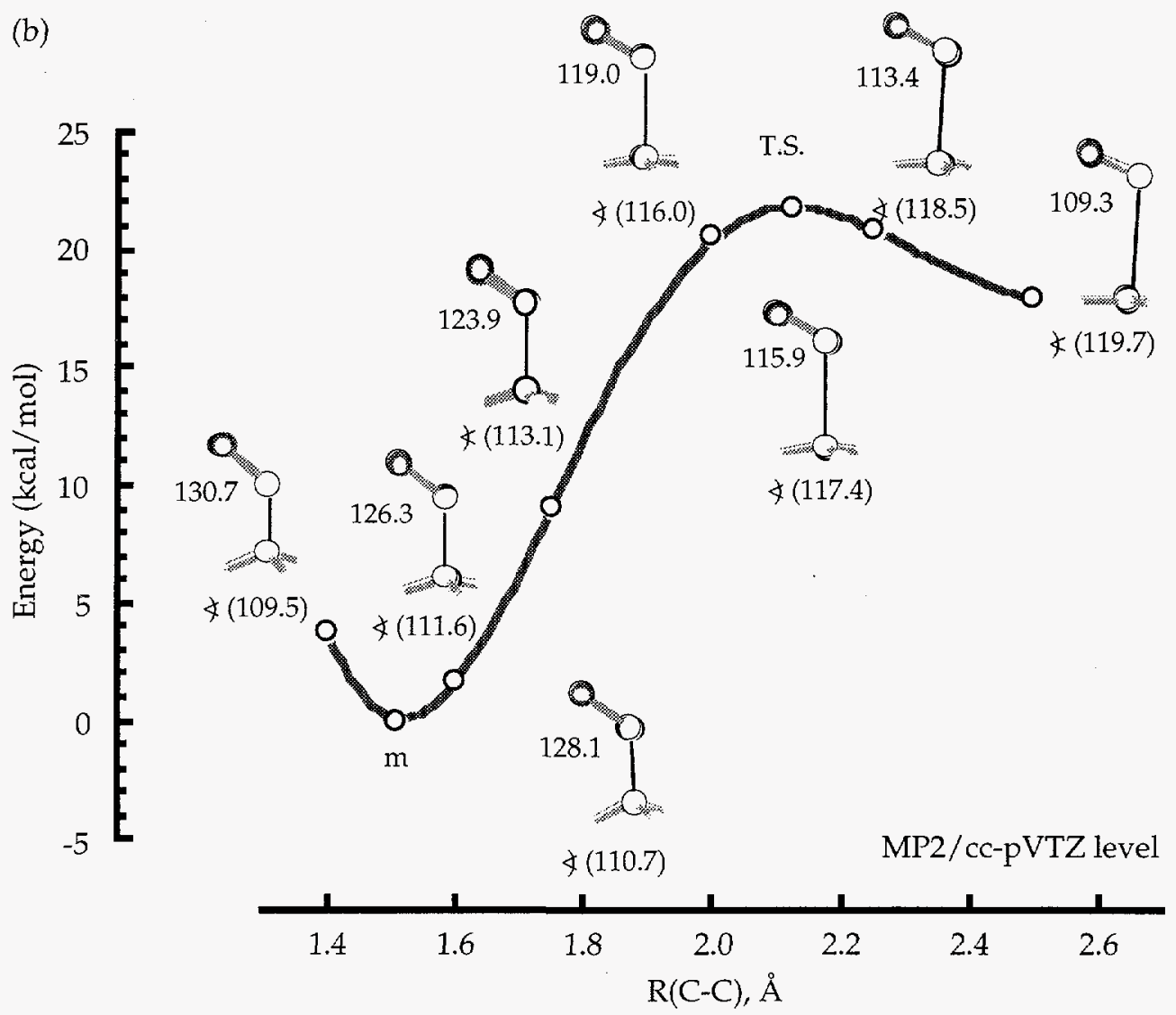

Figure 1.8. (a) Optimal structures of the minimum $\left({ }^{2} \mathrm{~A}^{\prime}\right)$ and transition state of acetyl radical and of $\mathrm{CH}_{3} \mathrm{COCl}\left({ }^{1} \mathrm{~A}^{\prime}\right)$ at the MP2/cc-pVTZ level of theory. The transition state is for dissociation of the acetyl radical and the arrows show the displacement of the atoms along the mode corresponding to the imaginary frequency at the transition state. Internal coordinates are indicated in degrees and Angstroms. (b) Energy profile for the $\mathrm{CH}_{3} \mathrm{CO}$ dissociation along the $\mathrm{C}-\mathrm{C}$ reaction coordinate at the MP2/cc-pVTZ level of theory. The values of the $(\mathrm{C}-\mathrm{C}-\mathrm{O})$ and $(\mathrm{H}-\mathrm{C}-\mathrm{H})$ angles are indicated for each value of the $\mathrm{C}-\mathrm{C}$ bond. $\mathrm{m}$ : minimum, T.S.: transition state. 
of the pyramid. The $C_{3}$ axis of symmetry passes through the center of the equilateral triangle formed by the three oxygen atoms and the top of the pyramid (fluoride ion). The base of the pyramid resembles the structure of the water trimer with the exception that all three otherwise "free" [in $\left(\mathrm{H}_{2} \mathrm{O}\right)_{3}$ ] hydrogens are pointing "up" toward the fluoride ion. The interoxygen separation in the pyramidal structure is $3.798 \AA$ (Hartree-Fock) and $3.247 \AA$ (MP2) respectively. Compared with the interoxygen separation in the water trimer ${ }^{39,40}$ we note an elongation of $0.9 \AA$ (Hartree-Fock) and $0.45 \AA$ (MP2). The second minimum for the $n=3$ cluster has $C_{s}$ symmetry and resembles a "ring" structure with two water molecules acting as both proton donors (to the ion) and proton acceptors and the third water molecule acting as a double donor to the first two. The symmetry plane passes through the ion and the oxygen of the double donor fragment $\left(\mathrm{O}_{\mathrm{c}}\right)$. The $\mathrm{C}_{\mathrm{s}}$ minimum is $2.1 \mathrm{kcal} / \mathrm{mol}$ above the $\mathrm{C}_{3}$ minimum.

The transition states corresponding to the isomerization between the $\mathrm{C}_{5}(n=1), \mathrm{C}_{1}(n=2)$, and $\mathrm{C}_{3}$ $(n=3)$ minima of the trimer have $\mathrm{C}_{2 \mathrm{v}}, \mathrm{C}_{2 \mathrm{~h}}$, and $\mathrm{C}_{3 \mathrm{~h}}$ symmetries, respectively. The electronic energy
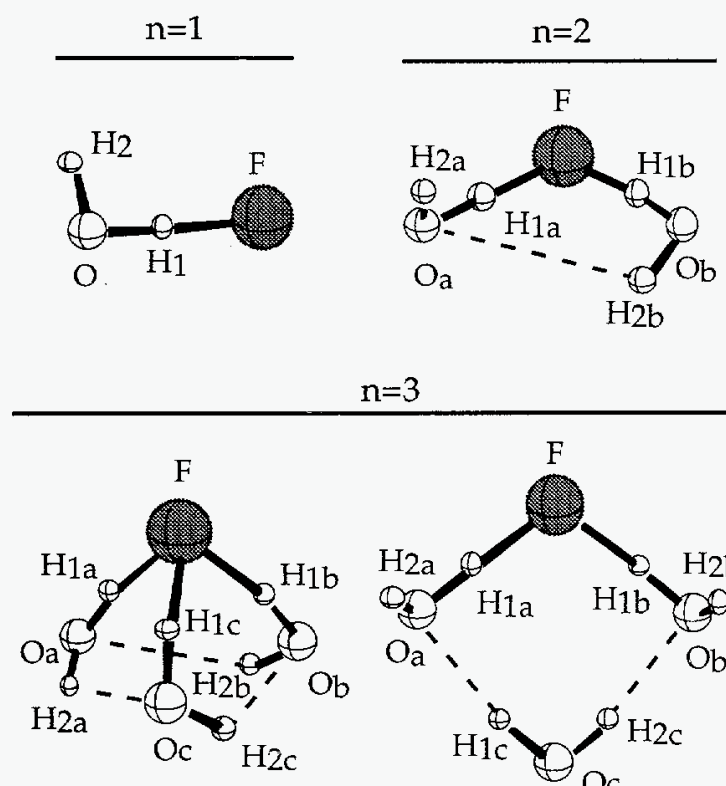

$\mathrm{n}=3$

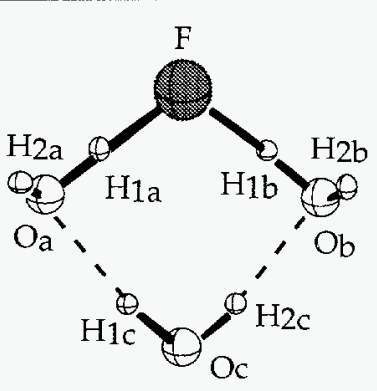

C3

Cs

Figure 1.9. Optimal geometries of the $\mathrm{F}^{-}\left(\mathrm{H}_{2} \mathrm{O}\right)_{n}$, $n=1-3$ clusters. difference between the minima and the transition states in which the ion is surrounded by water molecules is, however, small $(0.5-1.5 \mathrm{kcal} / \mathrm{mol})$ as seen from Table 1.6. When zero-point vibrational energy corrections are included, the energy difference becomes negligible and, in the $n=3$ case, it is reversed making the symmetric structure favorable by $0.1 \mathrm{kcal} / \mathrm{mol}$. It is therefore concluded that the dynamic rather than the static features of the potential energy surface determine the most probable orientation of the water molecules around the ion. This suggests that the concept of an "optimal geometry" has little meaning for these non-rigid systems. The location of the various minima on the $n=4$ potential energy surface would probably not provide much insight as the actual structures of these clusters at some finite temperature could be far away from the minimum energy configurations. The energy differences between the critical points were analyzed in terms of the relative magnitudes of the two- and higher-order interaction energy terms.

Table 1.6. Electronic $\left(\Delta \mathrm{E}_{\mathrm{e}}\right)$ energy difference between various critical points on the $n=1-3$ potential energy surfaces with the aug-cc-pVDZ set. $\Delta \mathrm{E}_{0}$ values include zero-point vibrational energy corrections. Values in parentheses are based on single point MP4 energy calculations at the corresponding optimal geometries at the MP2 level. T.S.= transition state, $\mathrm{Min}=$ minimum.

\begin{tabular}{|c|c|c|c|c|}
\hline \multirow[b]{2}{*}{$\mathrm{n}$} & & \multicolumn{3}{|c|}{ Energy $(\mathrm{kcal} / \mathrm{mol})$} \\
\hline & & $\mathrm{HF}^{a}$ & MP2 & MP4 \\
\hline \multirow[t]{2}{*}{1} & $\mathrm{C}_{2 \mathrm{v}}$ (T.S.) $-\mathrm{C}_{\mathrm{s}}(\mathrm{Min})$ & $5.6^{b}$ & 7.5 & $(7.0)$ \\
\hline & & $5.5^{c}$ & 7.7 & $(7.2)$ \\
\hline \multirow[t]{2}{*}{2} & $\mathrm{C}_{2 \mathrm{~h}}$ (T.S.) $-\mathrm{C}_{1}(\mathrm{Min})$ & -0.01 & 0.45 & $(0.57)$ \\
\hline & & -0.08 & 0.04 & $(0.16)$ \\
\hline \multirow[t]{4}{*}{3} & $\mathrm{C}_{3 \mathrm{~h}}(\mathrm{~T} . \mathrm{S})-.\mathrm{C}_{3}(\mathrm{Min})$ & 0.06 & 1.63 & $(2.03)$ \\
\hline & & -0.01 & -0.10 & $(0.30)$ \\
\hline & $\mathrm{C}_{\mathrm{s}}(\mathrm{Min})-\mathrm{C}_{3}(\mathrm{Min})$ & 2.5 & 1.4 & $(1.76)$ \\
\hline & & 2.9 & 1.1 & $(2.06)$ \\
\hline
\end{tabular}


$A b$ Initio Studies of $\mathrm{M}^{+}\left(\mathrm{H}_{2} \mathrm{O}\right)_{n}$ and $\mathrm{M}^{2+}\left(\mathrm{H}_{2} \mathrm{O}\right)_{n}$ Clusters (D. F. Feller, E. D. Glendening, ${ }^{(a)}$ K. A. Peterson, ${ }^{(b)}$ D. E. Woon, ${ }^{(a)}$ and R. A. Kendall $\left.{ }^{(c)}\right)$

(a) AWU Postdoctoral Research Associate

(b) Joint appointment with Department of Chemistry, Washington State University

(c) High Performance Computational Chemistry Group

Reliable theoretical data on the structure and energetics of small water clusters containing alkali or alkaline earth ions $\left(\mathrm{Li}^{+}\right.$through $\mathrm{Cs}^{+}$and $\mathrm{Mg}^{2+}$ through $\mathrm{Ra}^{2+)}$ are of interest due to the widespread appearance of such systems in nature and the difficulty of obtaining this information experimentally. Furthermore, the data is of interest because the observed binding preferences of crown ethers for particular cations hinge on a competition between water-ion and crown etherion interactions which is most easily determined theoretically.

The geometries, binding energies, and enthalpies of molecular clusters incorporating a single metal ion and up through six waters were determined by using basis sets and levels of theory comparable to those employed in a parallel study of ion/18-crown- 6 systems. The highest such level utilized the polarized $6-31+\mathrm{G}^{*}$ basis set and second order perturbation theory. To estimate the inherent accuracy of the MP2/6-31+G* level of theory, some of the same clusters were re-examined with more elaborate methods. This calibration procedure involved the use of the correlation consistent sequence of basis sets to estimate the complete basis set limit, coupled with higher order correlation methods (e.g., MP4 and CCSD(T)). Representative results for $\mathrm{Li}^{+}\left(\mathrm{H}_{2} \mathrm{O}\right)$ and $\mathrm{Na}^{+}\left(\mathrm{H}_{2} \mathrm{O}\right)$ are shown in Figure 1.10. Excellent agreement is observed between counterpoise (CP) corrected $6-31+G^{*}$ results and the best available theoretical values. Slightly poorer agreement is seen in the metal-oxygen distance because of the lack of a basis set superposition correction. In general the effects of correlation recovery on the basic cation-water distance and binding energy is small $(\sim 0.03 \AA$ and $\sim 2$ $\mathrm{kcal} / \mathrm{mol}$, respectively). However, as the clusters increase in size and the potential for multiple water-water interactions increases, the importance of correlation effects also increase.

Core-valence correlation effects were found to be significant for alkali atoms ( $\mathrm{Na}, \mathrm{K}$, etc.). Selected calculations in this study included relativistic effects for heavier elements via relativistic effective core potentials. The accuracy of the effective core potentials was checked with all electron calculations that included the two dominant relativistic contributions to the Breit-Pauli Hamiltonian through first order in perturbation theory.

Path Integral Monte Carlo Simulations of Ionic Clusters (H. Gai, ${ }^{(a)}$ L. X. Dang, G. K. Schenter, and B. C. Garrett)

(a) AWU Postdoctoral Research Associate

Examination of the chemical physics of ionic clusters provides a molecular level understanding of solvation phenomena. The experimental, computational, and theoretical studies of these systems allow us to investigate the specific size effects (i.e., the sequential buildup of the solvation shell in various size clusters) and extrapolate further details of the nature of ionic solvation in aqueous solutions. The motivation of our work is to systematically study the factor affecting the structure and energetics of aqueous ionic clusters. Earlier we reported a classical molecular dynamics simulation of the structural and energetic properties for the ionic clusters $\mathrm{Cl}^{-}\left(\mathrm{H}_{2} \mathrm{O}\right)_{n}, \mathrm{n}=1$ 6.53 In that study, we took many-body interactions into account and found that they play an important role in determining the properties of ionic clusters. Another important factor that we wish to include is the quantum mechanical description of nuclear motion that can affect the enthalpies of binding and the structural properties of ionic clusters. In this work, we investigate these quantum effects by carrying out a series of path integral Monte Carlo simulations ${ }^{54}$ on the ionic clusters $\mathrm{Cl}^{-}\left(\mathrm{H}_{2} \mathrm{O}\right)_{n}, \mathrm{n}=1-6$, using the same polarizable models as reported in our earlier work. 53,55

Figure 1.11 contains the calculated binding enthalpies at $300 \mathrm{~K}$ using classical Monte Carlo (MC), path-integral MC (PIMC, denoted $\mathrm{MC}$ (quantum) in the figure), and molecular dynamics (MD) along with the corresponding experimental measurements. We observed that the quantum effects on the energetic property are rather small but measurable. Further work will include the study of quantum effects on the structural property as well as simulations at a variety of temperature. 


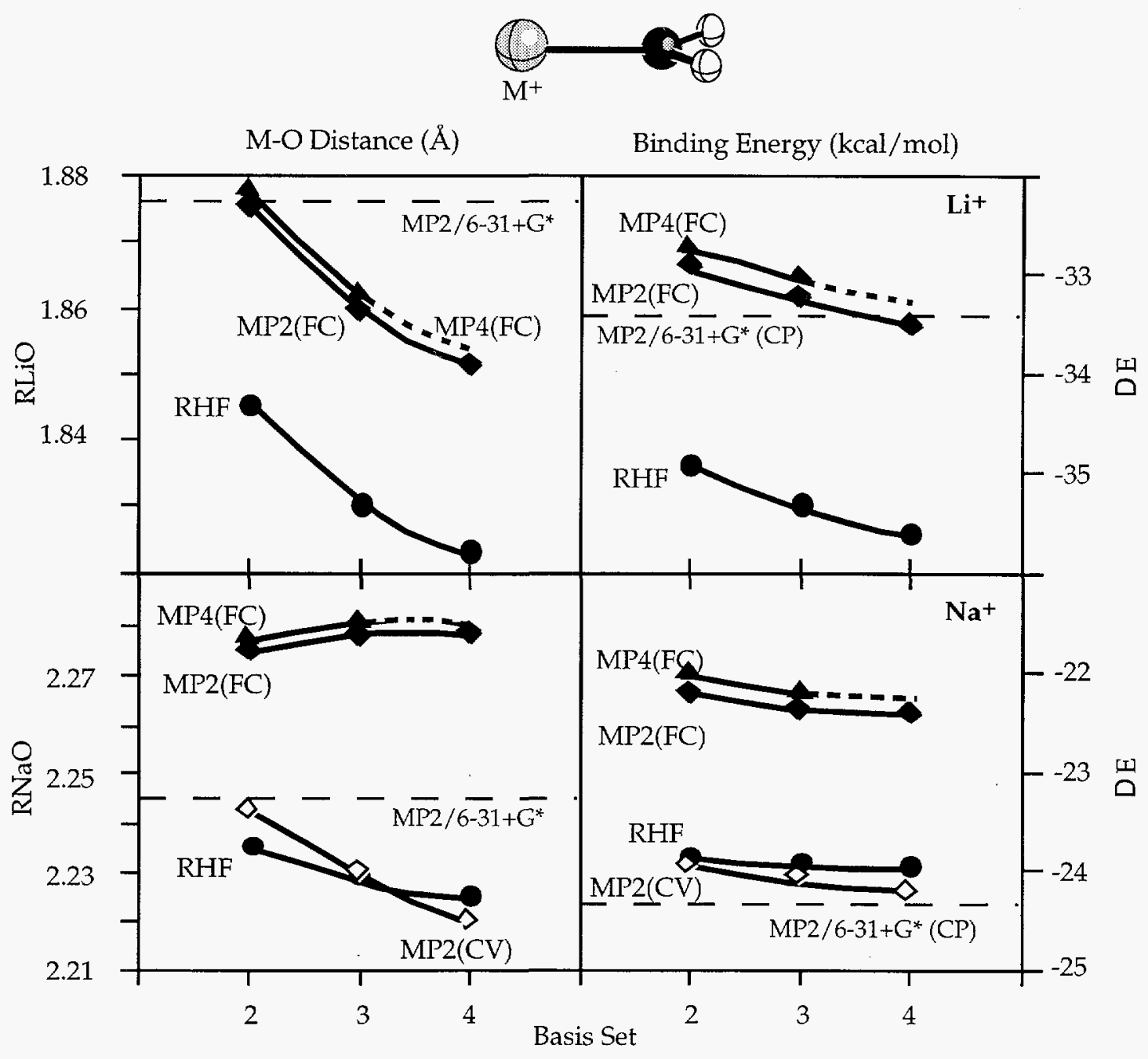

Figure 1.10. Convergence of the $\mathrm{Li}^{+}\left(\mathrm{H}_{2} \mathrm{O}\right)$ and $\mathrm{Na}^{+}\left(\mathrm{H}_{2} \mathrm{O}\right)$ metal-oxygen distance and binding energy as a function of the basis set completeness and degree of correlation recovery. Basis sets 2,3 , and 4 are ccpVDZ/cc-pVDZ, cc-pVTZ/cc-pCVTZ, and cc-pVQZ/cc-pCVQZ on the cation and aug-cc-pVDZ, aug-cc$\mathrm{pVTZ}$, and aug-cc-PVQZ on water.

The Hydrogen Bond Energy of the Water Dimer (D. Feller, M. W. Feyereisen, ${ }^{(a)}$ and D. A. Dixon $^{(b)}$ )

(a) Cray Research, Inc., Egan, Minnesota

(b) DuPont Central Research and Development, Wiliminton, Delaware

Hydrogen bonding plays a critical role in a wide range of chemical and biological phenomena. It is also important for describing the behavior of many synthetic materials. The prototypical hydrogen bond, and the one which has received the most theoretical attention, is that found between two water molecules. The small size of the water dimer and the difficulty in obtaining experimental results for this species make it an ideal candidate for $a b$ initio electronic structure studies. Large scale $a b$ initio molecular orbital calculations on the binding energy of the water dimer have been performed. These calculations extend the previous correlation consistent basis set work ${ }^{56}$ to include larger basis sets and core/valence correlation effects have now been included. The calculations with the large basis sets were done with the resolution of the identity MP2 (RI-MP2) method. ${ }^{57}$ The RI-MP2 method rapidly converges to the conventional MP2 technique as the size of a fitting basis which is used by the new 


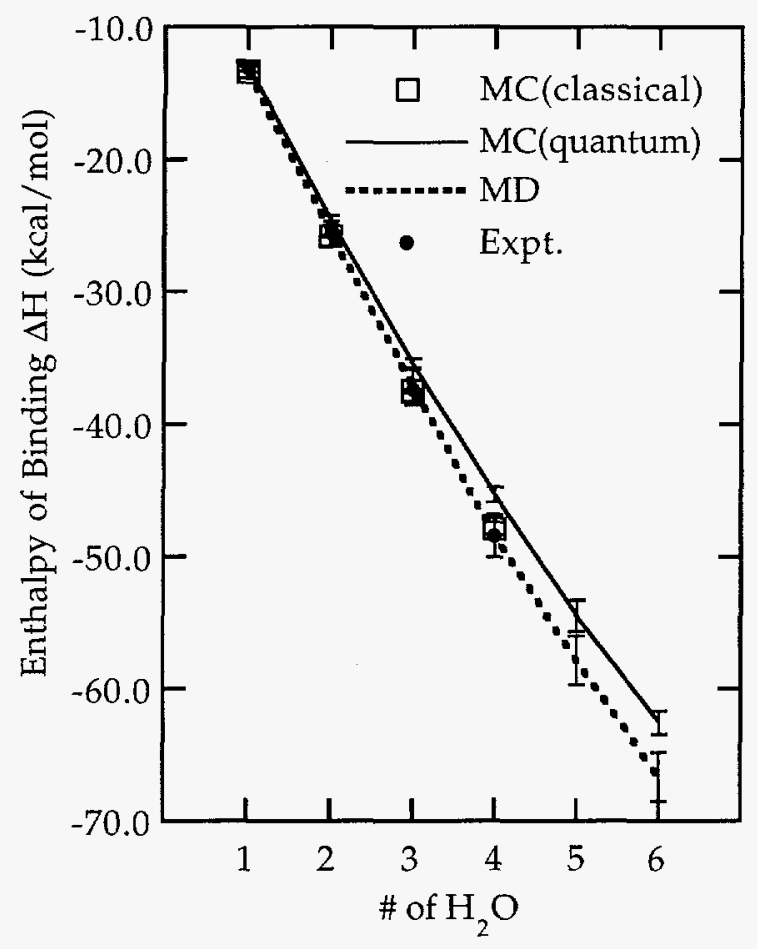

Figure 1.11. Binding enthalpies for the ionic clusters obtained using different techniques.

method, approaches completeness. This allows one to evaluate MP2-like energies with basis sets as large as the aug-cc-pV5Z basis set in an acceptable period of time. This basis set is a $[7 \mathrm{~s}, 6 \mathrm{p}, 5 \mathrm{~d}, 4 \mathrm{f}, 3 \mathrm{~g}, 2 \mathrm{~h} /-6 \mathrm{~s}, 5 \mathrm{p}, 4 \mathrm{~d}, 3 \mathrm{f}, 2 \mathrm{~g}]$ contraction totaling 574 functions for the water dimer. The results are summarized in Table 1.7. The present work confirms the earlier estimate of -4.9 $\mathrm{kcal} / \mathrm{mol}$ as the MP2(FC) basis set limit. Core/valence correlation effects are found to increase the binding energy by $\sim 0.05 \mathrm{kcal} / \mathrm{mol}$. The best estimate of the electronic binding energy of the water dimer is $-5.0 \pm 0.1 \mathrm{kcal} / \mathrm{mol}$. Correcting this value for zero-point and temperature effects yields a value for $\Delta \mathrm{H}(375)=-3.2 \pm 0.1$ $\mathrm{kcal} / \mathrm{mol}$. This value is within the error limits of the best experimental estimate ${ }^{49}$ of $-3.6 \pm 0.5$ $\mathrm{kcal} / \mathrm{mol}$ with the calculations favoring the lower end of the experimental energy range. It should be useful to adopt the present estimate in empirical and semiempirical model potentials.
Table 1.7. Interaction energies $(\mathrm{kcal} / \mathrm{mol})$ for the water dimer.

\begin{tabular}{clcc} 
Basis Set & Method & $\Delta \mathrm{E}$ & $\begin{array}{c}\Delta \mathrm{E} \\
\left(\mathrm{BSSE}^{a}\right)\end{array}$ \\
\hline cc-pVDZ & RI-MP2(FC) & -7.54 & -4.25 \\
& MP2(FC) & -7.29 & -3.94 \\
cc-pVTZ & RI-MP2(FC) & -6.08 & -4.45 \\
& MP2(FC) & -6.03 & -4.40 \\
cc-pVQZ & RI-MP2(FC) & -5.47 & -4.68 \\
& MP2(FC) & -5.45 & -4.67 \\
cc-pV5Z & SCF & -3.67 & -3.57 \\
& RI-MP2(FC) & -5.09 & -4.80 \\
& & & \\
aug-cc- & RI-MP2(FC) & -5.31 & -4.56 \\
pVDZ & MP2(FC) & -5.16 & -4.32 \\
aug-cc- & RI-MP2(FC) & -5.18 & -4.69 \\
pVTZ & MP2(FC) & -5.10 & -4.64 \\
aug-cc- & RI-MP2(FC) & -5.09 & -4.82 \\
pVQZ & MP2(FC) & -5.03 & $-4.79 b$ \\
aug-cc- & RI-MP2(FC) & -4.98 & \\
pV5Z & & & \\
aug-cc- & RI-MP2(FC) & -5.33 & -4.57 \\
pCVDZ & RI-MP2 & -5.42 & -4.58 \\
& MP2 & -5.25 & -4.34 \\
aug-cc- & MP4 & -5.30 & -4.29 \\
pCVTZ & RI-MP2(FC) & -5.04 & -4.69 \\
& RI-MP2 & -5.09 & -4.72 \\
\hline
\end{tabular}

a Basis set superposition error estimated using the $\mathrm{CP}$ correction.

$b$ Estimated from the CP correction for the augcc-pVQZ basis.

\section{Modeling Molecular Processes in Solution}

Solution-phase chemistry plays a central role in the Molecular Theory and Modeling program. The effort is focused on the structure, energetics, and dynamics of molecular processes in aqueous solutions. This work is the basis for an enhanced understanding of molecular processes occurring in contaminated and natural groundwater and in complex waste solutions. This core program in solution chemistry is designed to further the understanding of the fundamental molecular processes occurring in solution (e.g., solvation and reaction), in order to provide a basis for the development of accurate techniques for simulating the behavior of complex waste solutions. This work includes studies of ionic solvation, supercritical fluids, and solvent effects on chemical reactions. 
Ionic Association Thermodynamics (D. E. Smith $^{(a)}$ and L. X. Dang)

(a) AWU Postdoctoral Research Associate; present address is Department of Chemistry, New Mexico State University

The thermodynamics of ionic association contributes significantly to condensed-phase chemical reaction thermodynamics, and plays a role in the structure and function of a variety of biochemical systems. ${ }^{58}$ The association free energy, referred to as the potential of mean force (PMF), of a strontium-chloride ion pair in water has been calculated by using classical molecular dynamics. ${ }^{59}$ The calculated PMF is displayed in Figure 1.12 along with the PMF for calcium chloride. There are well defined contact ion pair (CIP) and solvent-separated ion pair (SSIP) minima for both systems. The CIP minimum is shifted out and stabilized for strontium chloride relative to calcium chloride, while the SSIP minimum is shifted out and destabilized. The barrier to CIP dissociation is also reduced for calcium chloride. These trends are consistent with both theoretical and experimental measurements on similar systems, ${ }^{60}$

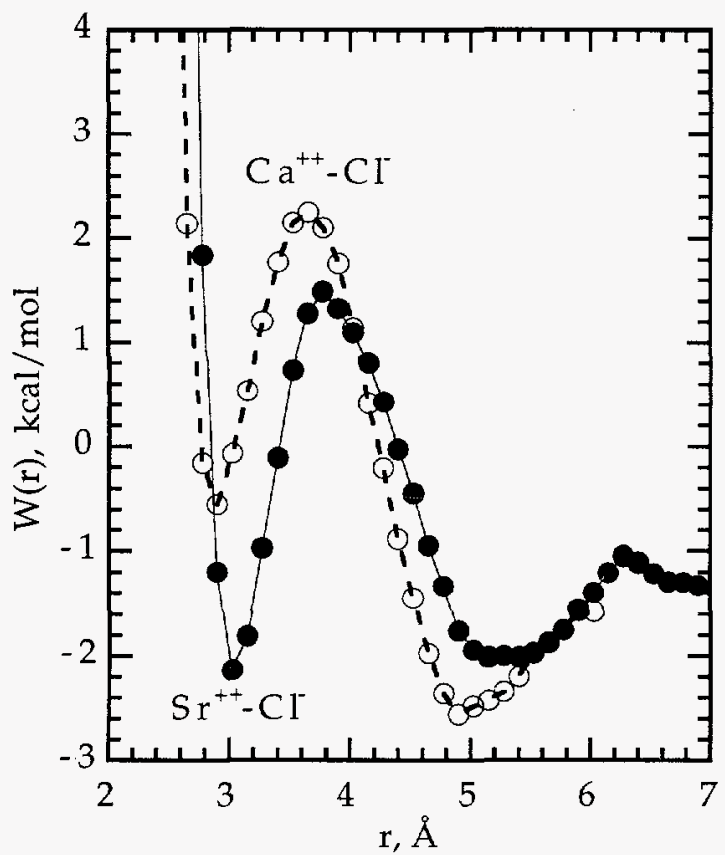

Figure 1.12. The calculated potential of mean force for the $\mathrm{Sr}^{++}-\mathrm{Cl}^{-}$and $\mathrm{Ca}^{++}-\mathrm{Cl}^{-}$ion pairs in water. and may be explained in terms of the larger size and weaker ion-water interaction of $\mathrm{Sr}^{+2}$ relative to $\mathrm{Ca}^{+2}$.

Solvent Effects on Metal Cation-Aromatic Interactions - A Molecular Dynamics Study (T.-M. Chang ${ }^{(a)}$ and L. X. Dang)

(a) AWU Postdoctoral Research Associate

Interactions between metal cations and aromatic residues are of fundamental importance in hostguest chemistry and in biological systems. We studied the solvent effect on the benzene- $\mathrm{K}^{+}$association in water using the molecular dynamics simulation technique. The potential parameters for $\mathrm{K}^{+}$are taken from previous work, and the potential parameters for benzene are taken from Jorgensen ${ }^{61}$ with slight modification to reproduce the experimental gas phase benzene- $\mathrm{K}^{+}$binding energy. Figure 1.13(a) displays the benzene- $\mathrm{K}^{+}$ gas phase interaction potential as a function of the distance between $\mathrm{K}^{+}$and the center of mass of benzene. The PMF for benzene- $\mathrm{K}^{+}$interaction in water is calculated using the free energy perturbation method ${ }^{62}$ and is depicted in Figure 1.13(b). The PMF results clearly show two minima at a benzene- $\mathrm{K}^{+}$separation of 3.2 and 6.8 $\AA$, corresponding to a contact pair and a solvent separated pair (SSP), respectively. The contact pair corresponds to a geometry in which the $\mathrm{K}^{+}$is sitting above the plane of the benzene ring. The SSP is $0.14 \mathrm{kcal} / \mathrm{mol}$ more stable than contact pair and the dissociation barrier from contact pair to SSP is $1.04 \mathrm{kcal} / \mathrm{mol}$. Figure 1.13 shows that the solvent plays an important role in benzene- $\mathrm{K}^{+}$ association in water.

\section{Modeling Molecular Processes in Separations Chemistry}

Research in separations chemistry concentrates on predicting the structure and energetics of ionligand complexes (such as crown ethers) and the dynamics of complex formation in aqueous solutions. The goal of this effort is to discover the factors that control the selectivity and efficiency of ligating species important in the pretreatment of nuclear and chemical wastes. 

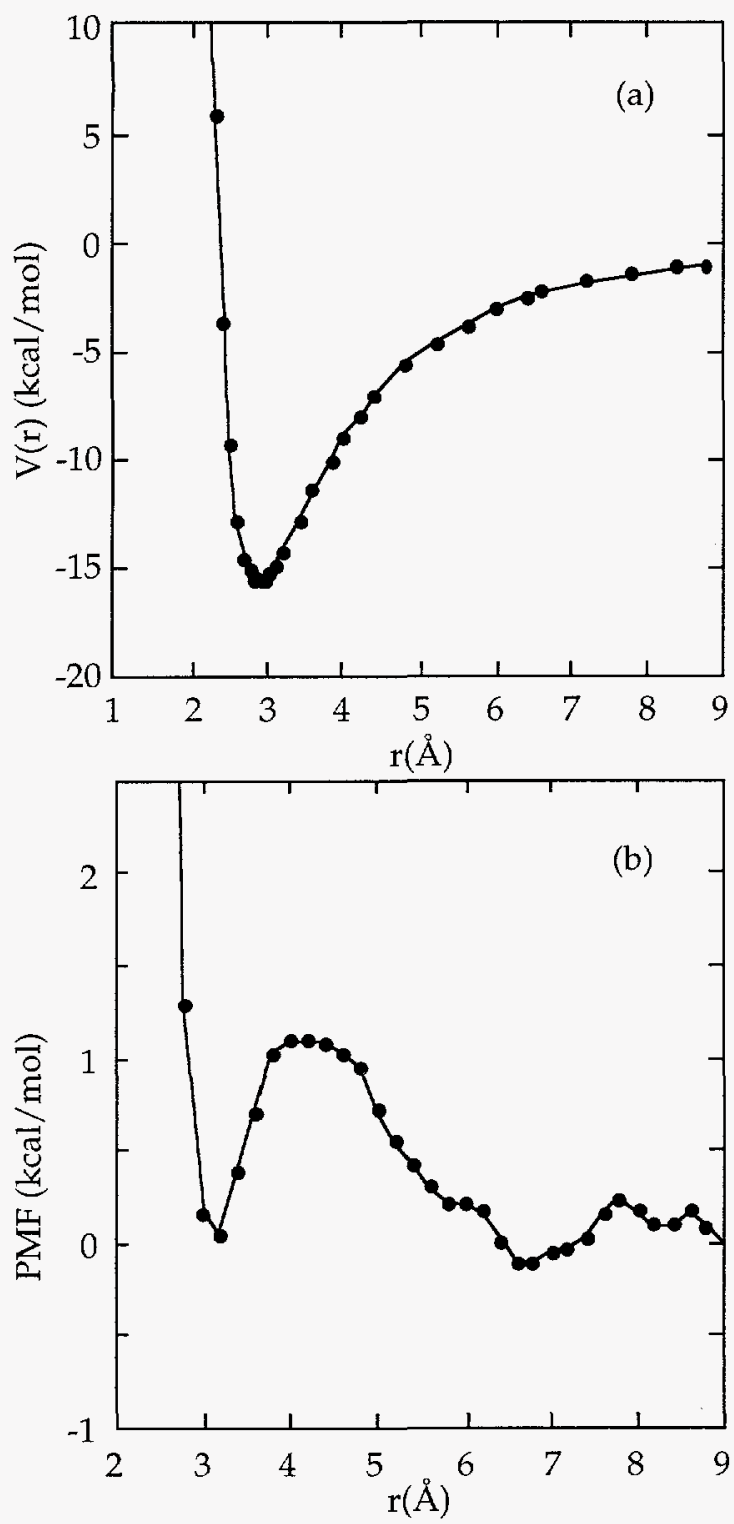

Figure 1.13. (a) Intermolecular potential of benzene and $\mathrm{K}^{+}$. (b) Potential of mean force for benzene and $\mathrm{K}^{+}$in water.

Crown ethers have drawn much experimental and theoretical interest since they were first described by Pederson in 1967.63 These cyclic polyethers can form remarkably stable complexes with alkali- and alkaline-earth metal cations. Furthermore, the selectivity of this binding is sensitive to macrocycle ring size, etherheteroatom, and macrocycle substituents. The ion specificity of crown-ethers makes them attractive as models for understanding enyzme chemistry as well as ligands useful to aid cleanup of mixed wastes that contain radionuclides such as cesium $\left(\mathrm{Cs}^{+}\right)$and strontium $\left(\mathrm{Sr}^{2+}\right)$, and transuranic ions.

An $A b$ Initio Investigation of the Selectivity of 18-crown-6 (S. E. Hill, ${ }^{(a)}$ E. D. Glendening, ${ }^{(b)}$ D. F. Feller, ${ }^{(c)}$ M. A. Thompson, and J. A. Nichols $^{(d)}$ )

(a) AWU Postdoctoral Research Associate

(b) Deptartment of Chemistry, Indiana State University

(c) Joint Appointment with the Computational Chemistry Environment Group

(d) High Performance Computational Chemistry Group

Clusters have been used to model the interaction of crown ethers with alkali and alkaline-earth cations using the following ab initio electronic structure methods: restricted Hartree-Fock (RHF) with 3-21G basis (denoted RHF/3-21G), RHF/6$31+G^{*}$ and $M P 2 / 6-31+G^{*}$. To judge the quality of our theoretical model, comparisons are made whenever possible with reliable experimental data. For example, 18-crown-6 is experimentally known to preferentially bind potassium over all other alkali metal cations in aqueous solution. On the other hand, our calculations on the simple gas phase complexation reactions,

$$
\mathrm{M}^{+}+18 \text {-crown-6 } \rightarrow \mathrm{M}^{+}(18-\text { crown-6), }
$$

on the other hand, predict that $\mathrm{Li}^{+}$is the most strongly bound ion and that the binding decreases monotonically from $\mathrm{Li}^{+}$to $\mathrm{Rb}^{+}$. The binding of the alkali ions to 18 -crown- 6 in aqueous solution involves a competition between the binding of the ion to water and to 18-crown-6. This process can be modeled by calculating the energetics of the exchange reactions

$$
\begin{gathered}
\mathrm{M}^{+}\left(\mathrm{H}_{2} \mathrm{O}\right)_{n}+\mathrm{M}^{\prime+}(18 \text {-crown-6) } \rightarrow \\
\mathrm{M}^{\prime}+\left(\mathrm{H}_{2} \mathrm{O}\right)_{n}+\mathrm{M}^{+}(18-\text { crown- } 6) \\
\mathrm{M}^{2+}\left(\mathrm{H}_{2} \mathrm{O}\right)_{n}+\mathrm{M}^{\prime 2+}(18-\text { crown-6) } \rightarrow \\
\mathrm{M}^{\prime 2+}\left(\mathrm{H}_{2} \mathrm{O}\right)_{n}+\mathrm{M}^{2+}(18 \text {-crown-6) }
\end{gathered}
$$

where $\mathrm{M}^{+}$and $\mathrm{M}^{\prime}$ are two different ions. The $a b$ initio trends are in good agreement with experiment, revealing a preference for potassium for +1 ions and barium for +2 ions. Figure 1.14 shows the effect of increasing the number of waters on the energetics. 

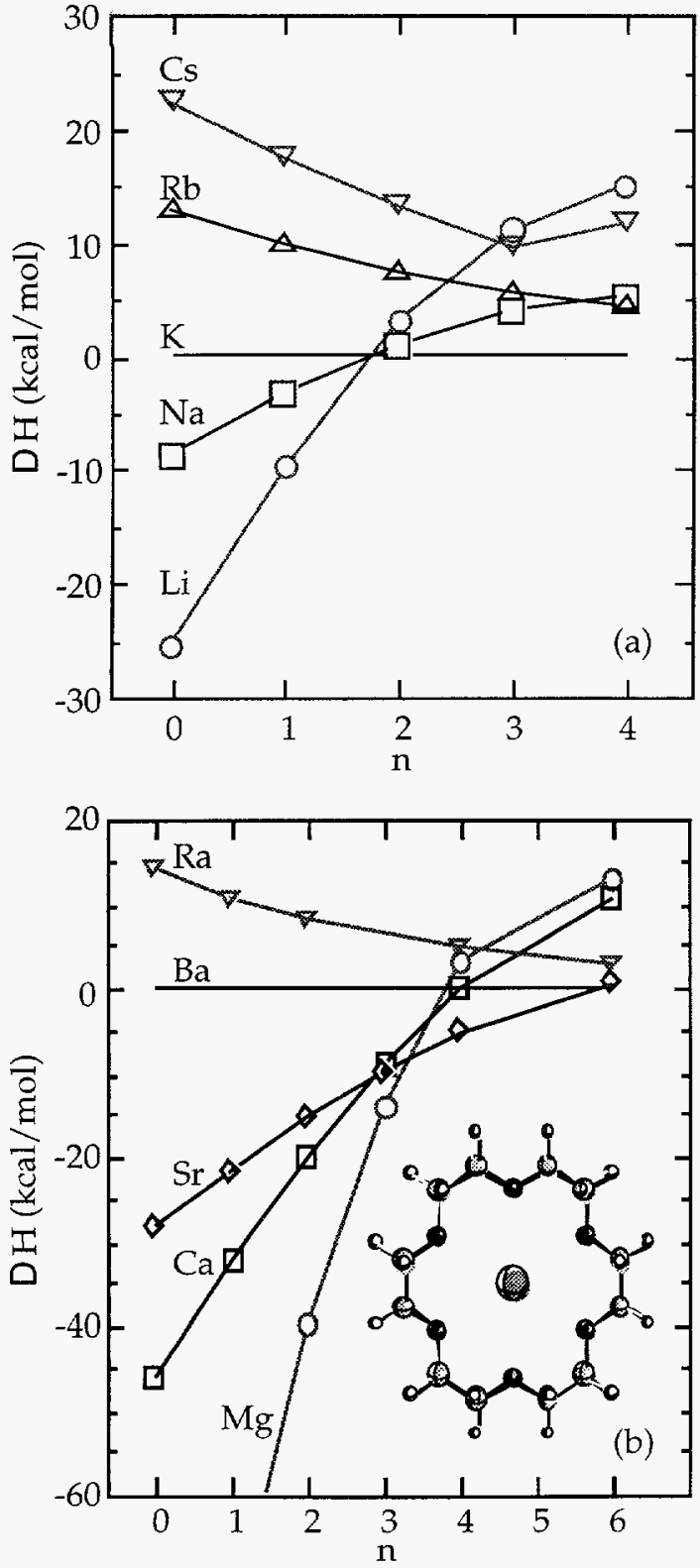

Figure 1.14. The effect of additional waters on the binding preference of 18-crown-6 at the $\mathrm{MP} 2 / 6-31+\mathrm{G}^{*}$ level. (a) Enthalpies for the reaction $\mathrm{K}^{+} / 18 \mathrm{c} 6+\mathrm{M}^{+}\left(\mathrm{H}_{2} \mathrm{O}\right)_{\mathrm{n}} \rightarrow \mathrm{K}^{+}\left(\mathrm{H}_{2} \mathrm{O}\right)_{\mathrm{n}}+$ $\mathrm{M}^{+} / 18 \mathrm{c}$. (b) Enthalpies for the reaction $\mathrm{B}^{2+} / 18 \mathrm{c} 6$ $+\mathrm{M}^{2+}\left(\mathrm{H}_{2} \mathrm{O}\right)_{\mathrm{n}} \rightarrow \mathrm{Ba}^{2+}\left(\mathrm{H}_{2} \mathrm{O}\right)_{\mathrm{n}}+\mathrm{M}^{2+} / 186$.

Due to the time-consuming nature of large basis set, correlated calculations, several computationally cheaper alternatives were investigated. Density functional theory with nonlocal gradient corrections was found to reproduce the conventional MP2 cation-ether geometries and binding energies with remarkable accuracy in a fraction of the time. The same was true of an approximation to MP2 known as resolution of the identify MP2. Thus, both of these methods offer promise for future work.

Combined $A b$ Initio and Experimental Studies of Small Ether/Cation Clusters (D. F. Feller ${ }^{(a)}$ E. D. Glendening, ${ }^{(b)}$ D. Ray, ${ }^{(c)}$ P. Armentrout ${ }^{(d)}$ and M. Birke $\left.{ }^{(d)}\right)$

(a) Joint Appointment with the Computational Chemistry Environment Group

(b) Department of Chemistry, Indiana State University

(c) Chemical Structure and Dynamics Program, EMSL

(d) Department of Chemistry, University of Utah

The only previous direct experimental measurement of the gas-phase binding energy of alkali metal cations and 18-crown-6, by A. Katritzky et al., ${ }^{64}$ found values considerably smaller than our $a b$ initio results. For example, their ion cyclotron resonance binding enthalpy for $\mathrm{K}^{+}: 18$-crown- 6 at $350 \mathrm{~K}$ was $-40 \pm 8 \mathrm{kcal} / \mathrm{mol}$, compared to an MP2 value of $-72 \mathrm{kcal} / \mathrm{mol}$. To shed light on underlying sources of error in the theoretical numbers, a joint experimental/theoretical investigation was begun on the binding energies of alkali cations with small ethers (e.g., dimethyl ether, dimethoxy ethane, and 12-crown-4). The experimental study employed collision induced dissociation (CID) techniques, while the theoretical approach employed the same basis sets and levels of theory which were used in the earlier crown ether study. As seen in Figure 1.15, the counterpoise corrected $\mathrm{MP} 2 / 6-31+\mathrm{G}^{*}$ binding enthalpies are in reasonably good agreement for the dimethyl ether complexes and for a single dimethoxyethane. Greater discrepancies are observed for the complex containing two dimethoxyethanes and 12crown-4.

Development of Hybrid Quantum Mechanical/Molecular Mechanical Force Fields for Crown-Ethers (M. A. Thompson, E. D. Glendening, ${ }^{(a)}$ and D. Feller)

(a) AWU Postdoctoral Research Associate

To date, most theoretical studies of ion/crownether interactions have employed molecular mechanics (MM) potentials. These models have 


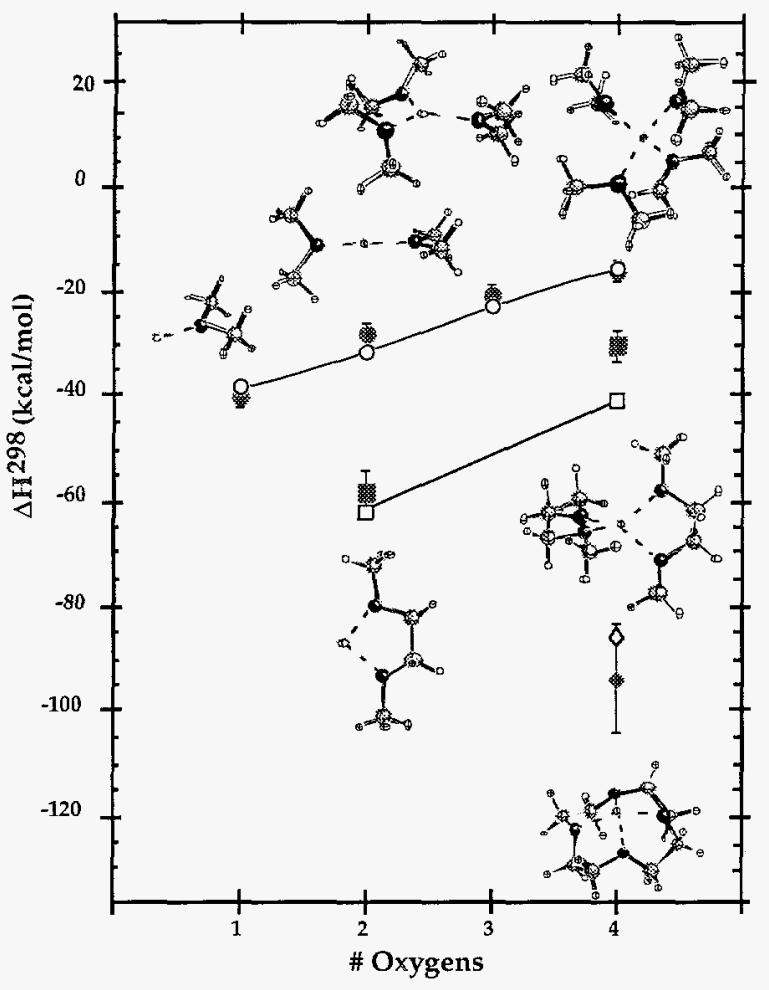

Figure 1.15. Comparison of CID and $a b$ initio MP2 theoretical predictions of the binding enthalpies of a variety of lithium/ether complexes.

generally been successful and have provided much insight. However, MM treatments are not generally capable of describing polarization and charge transfer effects. Furthermore, MM parameterization schemes often lack the generality for a wide range of chemical substituents that could be applied to the crown macrocycle as well as treatment of metal ions. Exploiting new theoretical and computational tools to better understand crown-ether chemistry in solution and suggest modifications to enhance affinity and specificity are the central goals of this research project. The crown-ether, 18-crown-6 $(18 \mathrm{c} 6)$, is treated as a quantum mechanical (QM) solute using both the AM1 and TAM1 (vide infra) model Hamiltonians. Water is treated with the MM SPC/e water model, $\mathrm{K}^{+}$is treated by the MM parameterization of Dang, ${ }^{55}$ and $\mathrm{CCl}_{4}$ is treated with the united atom potential of Rebertus et al. 65 The QM/MM coupling Hamiltonian, that allows for the $\mathrm{QM}$ and $\mathrm{MM}$ regions to interact, is treated in a fashion similar to Field et al. ${ }^{66}$ and Gao and Xia. ${ }^{67}$ The QM/MM method is implemented in the Argus computer program. 68 Molecular dynamics simulations are used to calculate average structural and energetic properties of $18 \mathrm{c} 6$, both free and complexed with $\mathrm{K}^{+}$, and in both aqueous and nonpolar solvents.

Initially, the QM/MM interaction Hamilttonian was parametrized to the binding energies and optimized geometries for $\mathrm{K}^{+} /$dimethylether (DME) using $a b$ initio Hartree-Fock and MP2/6$31+\mathrm{G}^{*}$ results. The resulting $\mathrm{QM} / \mathrm{MM}$ model describes the polarization response of both free DME and $\mathrm{K}^{+}$-complexed DME well. The $\mathrm{QM} / \mathrm{MM}$ model gives good agreement with the experimental and $a b$ initio structures for $\mathrm{K}^{+} / 18 \mathrm{c} 6$. Gas-phase $\mathrm{K}^{+} / 18 \mathrm{c} 6$ binding energies at the $\mathrm{QM} / \mathrm{MM}(70.2 \mathrm{kcal} / \mathrm{mol})$ and $\mathrm{MP} 2 / 6-31+\mathrm{G}^{*}(\mathrm{CP}$ corrected; $-72.0 \mathrm{kcal} / \mathrm{mol}$ ) levels agree well. Our simulation results for $\mathrm{K}^{+} / 18 \mathrm{c} 6$ in $\mathrm{H}_{2} \mathrm{O}$ show that the most probable $\mathrm{K}^{+} / 18 \mathrm{c} 6$ center of mass displacement is $0.25 \AA$, a result that is consistent with $\mathrm{K}^{+}$having an optimal "fit" for the cavity of $18 \mathrm{c} 6$. Still, we find that $\mathrm{K}^{+}$retains significant solvent accessibility coordinating two $\mathrm{H}_{2} \mathrm{O}$ molecules, on average, in the $\mathrm{K}^{+} / 18 \mathrm{c} 6$ simulation. The simulation average polarization energy for $18 \mathrm{c} 6$ interacting with both $\mathrm{K}^{+}$and the $\mathrm{H}_{2} \mathrm{O}$ solvent is $-14.9 \mathrm{kcal} / \mathrm{mol}$ which is $17 \%$ of the total electrostatic interaction energy. This result underscores the potential importance of quantum mechanics in describing the solution chemistry of ion-macrocycle interactions. ${ }^{69}$

The AM1 Hamiltonian generally does well in describing relative energies and geometries for selected rotamers of $18 \mathrm{c} 6$ (when compared to $a b$ initio Hartree-Fock/6-31+G* optimized geometries as well as $\mathrm{x}$-ray coordinates). However, rotational barriers for molecules like 1,2-dimethoxyethane (DME) (a model for a fragment of an oxygen-containing crown-ether) are usually underestimated by as much as $50 \%$ relative to experiment or high-level $a b$ initio calculations. ${ }^{70,71}$ To address this problem, we have developed the Torsion-modified AM1 (TAM1) Hamiltonian which employs MM-torsion terms to increase the accuracy of the OCCO and COCC rotamer energetics. The torsion terms are parameterized using the $a b$ initio calculations of Jaffe, Smith, and Yoon for 1,2-dimethoxyethane. ${ }^{70}$ We have used the TAM1 Hamiltonian in QM/MM studies of free $18 \mathrm{c} 6$ in both $\mathrm{H}_{2} \mathrm{O}$ and $\mathrm{CCl}_{4}$. The TAM1 method describes optimized geometries and relative energetics for $C_{i}$ and $D_{3 d}$ configurations of $18 \mathrm{c} 6$ slightly better than unmodified AM1. TAM1 simulation results of 
OCCO and COCC torsion angle distributions show $18 \mathrm{c} 6$ maintaining an oxygen-lined preorganized cavity in $\mathrm{H}_{2} \mathrm{O}$ in contrast to the simulation in $\mathrm{CCl}_{4}$. The radial distribution function $g(R)$ for the center of mass of $18 \mathrm{c} 6$ and the center of mass of water is shown in Figure 1.16. In addition the number of water molecules coordinating the crown ether, which is obtained by integration of $g(R)$, is shown. The coordination number for the first peak in $g(R)$ is approximately 2 , indicating that the crown-ether strongly binds two $\mathrm{H}_{2} \mathrm{O}$ molecules. These two waters are critical in maintaining the preorganized cavity of $18 \mathrm{c} 6$. Together, the two waters have a simulation average $18 \mathrm{c} 6 / \mathrm{H}_{2} \mathrm{O}$ stabilization energy of $-23.8 \mathrm{kcal} / \mathrm{mol}$, with each water maintaining two hydrogen bonds to oxygens of the crown-ether. These results are reported in more detail elsewhere. ${ }^{72}$

A Hybrid Quantum Mechanical/Molecular Mechanical Method that Includes MM Polarization: Application to Excited States of the Primary Donor of the Bacterial Photosynthetic Reaction Center (M. A. Thompson and G. K. Schenter)

We are interested in developing models of solvent effects useful for both ground and electronically excited states of quantum mechanical solutes in solution. To this end, a hybrid QM/MM model has been developed with polarizability in the $\mathrm{MM}$ region (QM/MMpol). A model system is defined in terms of QM particles consisting of the electrons and nuclei (or effective cores) and MM particles consisting of charged atomic centers with atom-centered point polarizabilities. Figure 1.17 shows the various interacting electrostatic components of the system. We write an effective Hamiltonian for this system

$$
\begin{aligned}
\hat{H}_{\text {sys }}= & \hat{H}_{\mathrm{QM}}^{0}+\hat{H}_{\mathrm{QMMM}}^{\mathrm{el}, \mathrm{stat}}+\hat{H}_{\mathrm{QMMM}}^{\mathrm{el}, \mathrm{pol}}+\hat{H}_{\mathrm{QMMM}}^{\mathrm{vdW}}+ \\
& \hat{H}_{\mathrm{MM}}^{\mathrm{el}, \mathrm{stat}}+\hat{H}_{\mathrm{MM}}^{\mathrm{el}, \mathrm{pol}}+\hat{H}_{\mathrm{MM}}^{\mathrm{bonded}}+\hat{H}_{\mathrm{MM}}^{\mathrm{vdW}}
\end{aligned}
$$

The above terms are, respectively: $\hat{H}_{\mathrm{QM}}^{0}$, the usual gas-phase time-independent, BornOppenheimer, non-relativistic QM Hamiltonian; $\vec{H}_{\text {OMMM }}^{\mathrm{el} \text {, stat }}$, the interaction between the QM system and the $\mathrm{MM}$ atom-centered charges; $\hat{H}_{\mathrm{QMMM}}^{\mathrm{el} \text {, }}$ the interaction between the $\mathrm{MM}$ atom-centered polarizable dipoles and the QM electrons and nuclei, respectively; $\widehat{H}_{\mathrm{MM}}^{\mathrm{el} \text {, stat }}$, the usual static MM

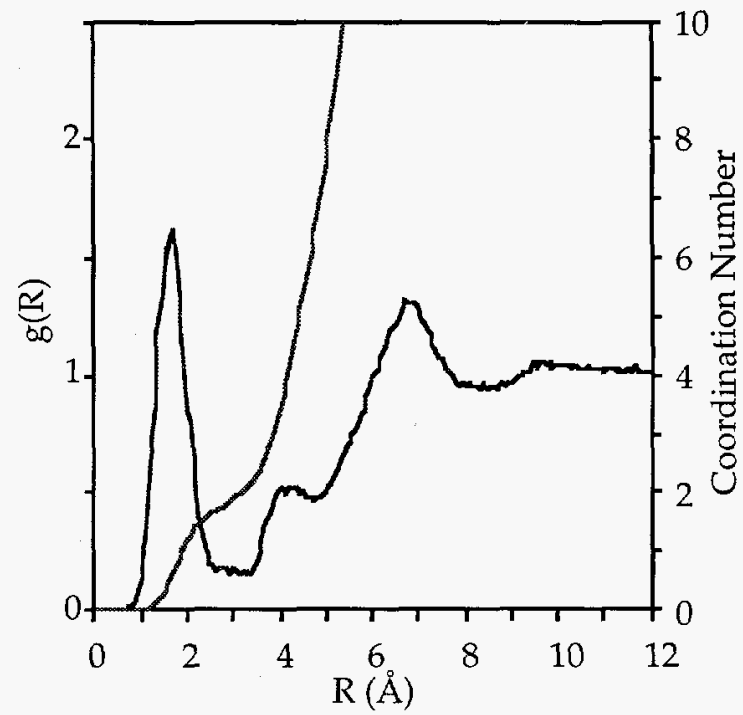

Figure 1.16. Radial distribution function for the separation of the $18 \mathrm{c} 6$ center-of-mass (COM) with $\mathrm{H}_{2} \mathrm{O}(\mathrm{COM})$ along with running coordination number. Results shown are for the TAM1 Hamiltonian. Analysis from configurations saved during a 300 ps simulation.

charge-charge interaction (coulomb) term; $\widehat{H}_{\mathrm{MM}}^{\mathrm{el}, \mathrm{pol}}$, the pure $\mathrm{MM}$ terms involving the MM polarizable dipoles including the point chargedipole interaction, the dipole-dipole interaction, and the self-energy for creating the dipoles; $\hat{H}_{\mathrm{MM}}^{\text {bonded }}$, the usual bonded terms for the MM interactions (i.e., bonds, bond angles, torsions, outof-plane, etc.); $\hat{H}_{\mathrm{OMMM}}^{\mathrm{vdW}}$ and $\hat{H}_{\mathrm{MM}}^{\mathrm{vdW}}$, the van der Waals interaction between $\mathrm{QM} / \mathrm{MM}$ and $\mathrm{MM} / \mathrm{MM}$ nonbonded atom pairs, respectively. Figure 1.17 clearly reveals, in pictorial form, the consistent treatment of the polarizable components in both the QM ( $\left.\Psi_{\text {sys }}\right)$ and the MM atomcentered polariable dipole $\{\mu\}$ regions, and suggests the role of the $\{\mu\}$ as a MM "wave function." Due to the operator terms $\bar{H}_{\mathrm{QM}}^{0}$, $\hat{H}_{\mathrm{OMMM}}^{\mathrm{el}, \mathrm{pol}}$, and $\hat{H}_{\mathrm{MM}}^{\mathrm{el}, \mathrm{pol}}$ that couple the QM and $M M$ regions, we solve a set of coupled SCF equations to obtain a mutually self-consistent $\Psi_{\text {sys }}$ and $\{\mu\}$ that together define a stationary state of our system. We have developed the formalism for both ground and electronic excited states.

The QM/MMpol method was applied to an analysis of the ground and electronic excited states of the bacteriochlorophyll $b$ dimer $(\mathrm{P})$ of the photo 


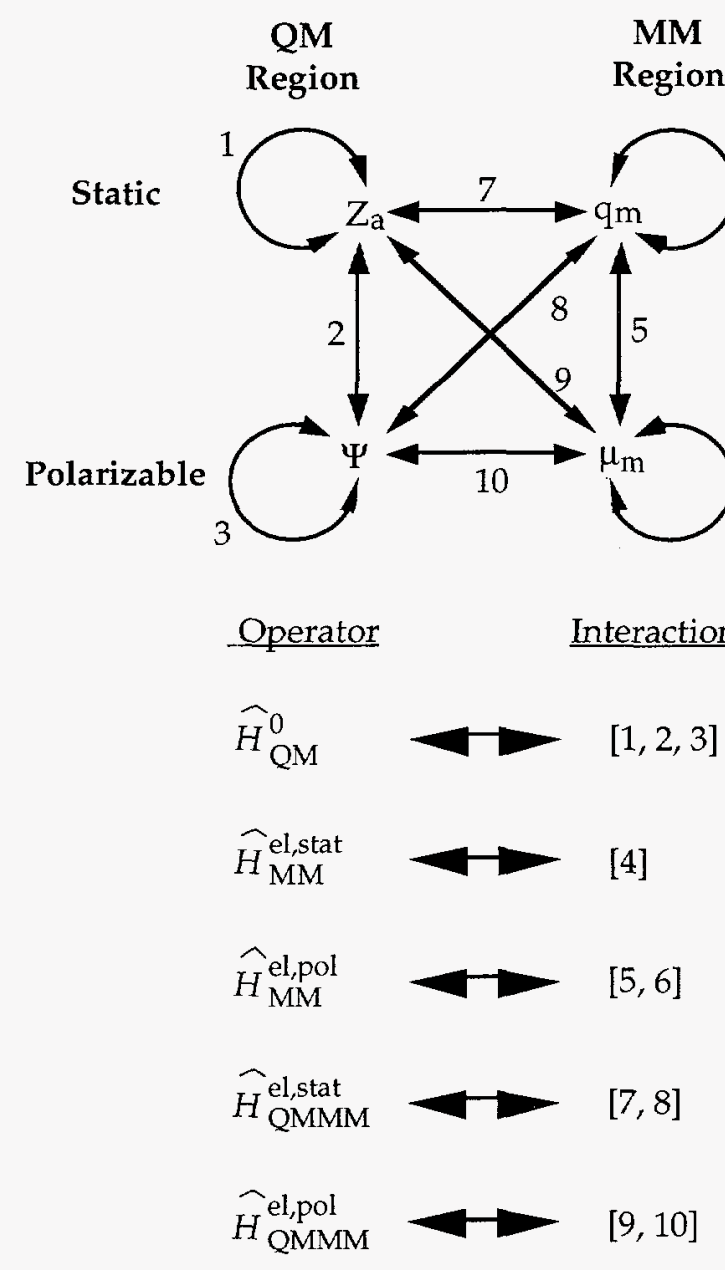

Figure 1.17. Schematic representation of the fundamental particles of our QM/MMpol system: $Z_{a}, Q M$ nuclei (or effective cores); $\Psi$, the $Q M$ wave function (electrons); $\mathrm{q}_{\mathrm{m}}, \mathrm{MM}$ atoms with charge $q$; and $\mu_{\mathrm{m}}$, the $\mathrm{MM}$ atomcentered polarizable dipoles. The interactions between the components are represented as numbered arrows which correspond to terms in our electrostatic system Hamiltonian (see text).

synthetic reaction center (RC) of Rhodopseudomonas viridis. This system was chosen for two reasons. The photosynthetic RC provides a good test of a large dye molecule in a complex environment. Also, this particular RC works at cryogenic temperature, thereby obviating the need to do molecular dynamics simulations. The coordinates of the heavy atoms for the $\mathrm{RC}$ are from the x-ray crystal structure reported by Deisenhofer and Michel. ${ }^{73}$ The QM region is treated with the semiempirical INDO/S method and the MM region is treated with fitted charges based upon the electrostatic potential from AMBER $^{74}$ and isotropic atomic polarizabilities from Applequist. ${ }^{75}$ The QM region consists of $P$ and the side chains of the five amino acids which interact directly with $P$. The MM region consists of the remainder of the protein, cofactors, and waters of crystallization. The resulting model of the $\mathrm{RC}$ consists of $325 \mathrm{QM}$ atoms embedded in the field of 20158 polarizable MM atoms. While dimer formation alone is enough to account for the majority of the monomer bacteriochlorophyll $b$ (BChlb) to $P$ redshift of the lowest electronic excited state of $P$ $\left(Q_{\mathrm{y} 1}\right), 76,77$ we demonstrate here that explicit treatment of the protein is required to properly interpret the experimental Stark data that describe the charge transfer asymmetry of $Q_{y 1}$. The static-charge potential from the MM model of the $R C$ alone causes $Q_{y 1}$ to have significantly better agreement with the Stark results than isolated $P$. However, consideration of the protein polarization potential is further required to obtain more complete agreement with Stark experiments. Thus we calculate: a $Q_{y 1}$ transition energy at $10826 \mathrm{~cm}^{-1}$ with a ground to excited state change in dipole moment of $4.8 \mathrm{D}$; an absorption Stark angle of $43^{\circ}$; a net shift of 0.15 electrons from the $\mathrm{L}$-subunit to the M-subunit of $\mathrm{P}$; and a linear dichroism angle (between the transition moment of $Q_{y 1}$ and the pseudo- $C_{2}$ axis of the $R C$ ) of $81^{\circ}$. These results are in good agreement with the experimental value of the Stark angle of $36.7 \pm 2^{\circ} .78$ 83 This is the first study, using a theoretical approach, to demonstrate the role of the protein in understanding the origins of the Stark effect of $P$.

Free Energy of Association of the $\mathrm{K}^{+}: 18-$ Crown6 Complex in Water: A Molecular Dynamics Study (L. X. Dang)

We report an extensive classical molecular dynamics simulation of the free energy profile for the complex $\mathrm{K}^{+}: 18-$ crown- 6 in water. The calculations included the counter ion $\mathrm{Cl}^{-}$and all longrange interactions were computed via the Ewald summation method. 84,85 The computed free energy profile in Figure 1.18 indicates that the minimum free energy is located at the crown ether center of mass and similar to the complex observed in the solid state or simulated in vacuo. In addition, the crown ether retains its $D_{3 \mathrm{~d}}$ geometry with small fluctuations throughout the simulation. The calculated binding free energy, and 


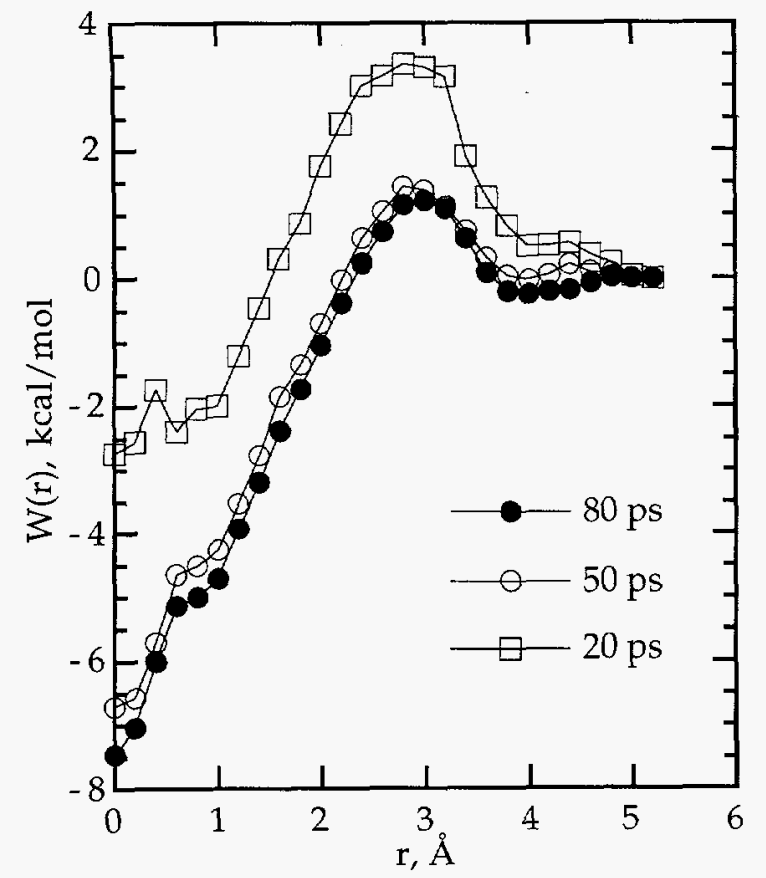

Figure 1.18. Free energies of association of the $\mathrm{K}^{+}$to 18 -crown-6 ether in water near $300 \mathrm{~K}$ as a function of the accumulation time.

the corresponding association constant, compare reasonably with experimental measurements made on the same system. Figure 1.18 also displayed the computed free energies as a function of the accumulation time. It is clear that the simulation with 50 ps or more of averaging for each ion-crown separation converges well. A snapshot of the complex $\mathrm{K}^{+}: 18$-crown-6 in water at the minimum of the free energy surface indicates two water molecules coordinating to the $\mathrm{K}^{+}$ ion from above and below the crown ether.

Mechanism and Thermodynamics of Ion Selectivity in Aqueous Solutions of 18-Crown-6 Ether: A Molecular Dynamics Study (L. X. Dang)

We have performed an extensive classical molecular dynamics simulation to examine the mechanism and thermodynamics for ion selectivity in aqueous solutions of 18-crown- 6 ether. 18crown-6 is one of the most extensively studied crown systems and the abundance of the experimental data for these systems provides a good prototype model to verify our approach. To the best of our knowledge, the present work is the first application of the potential of mean force approach to the evaluation of ion selectivity by crown ethers in aqueous solutions. Our simulations differ from previous work in that the free energies of complexation are computed as a function of the ion-crown separation, or PMF. This makes the current study valuable for several reasons. First, the computed PMF allows us to examine the minima positions of the complexes and they can be related directly to the x-ray data. The second valuable aspect is that we can compute the absolute binding energies by integrating over the PMFs, the resultants can be directly compared with the corresponding experimental measurements. The third advantage of our approach is that we can examine the role of solvent effects along the reaction coordinate (i.e., contact pair or solvent separated pair).

The free energy profiles or PMF for $\mathrm{M}^{+}: 18$-crown$6\left(\mathrm{M}^{+}=\mathrm{K}^{+}, \mathrm{Na}^{+}, \mathrm{Rb}^{+}, \mathrm{Cs}^{+}\right)$complexation are computed via the free energy perturbation method. Counterion effects are considered and all long-range interactions are computed via the Ewald summation method. 84,85 The resultant PMFs indicate that free-energy minima for the $\mathrm{K}^{+}$ and $\mathrm{Na}^{+}$ions are located at the crown ether center-of-mass as shown in Figure 1.19(a). Potassium is selected over sodium because of the greater free energy penalty associated with displacing water molecules from sodium as it approaches the crown ether. This leads to a substantial increase in the activation free energy and a decrease in the binding free energy for $\mathrm{Na}^{+}: 18-$ crown-6 complexation as compared to $\mathrm{K}^{+}: 18-$ crown-6. The selection of potassium over rubidium and cesium is due to the cation-size relative to the crown-ether cavity. The calculated PMFs for all the ions are shown in Figure 1.19(a). We computed binding free energies by integrating over the PMFs and the results are summarized in Figure 1.19(b). They are slightly underestimated; however, they follow the sequence $\mathrm{K}^{+}>\mathrm{Rb}^{+}>$ $\mathrm{Cs}^{+}>\mathrm{Na}^{+}$reported experimentally. ${ }^{86}$ The shortcoming in binding free energies can be overcome by improving the cation-crown ether interaction potential and/or taking in to account the nonadditive effects in these molecular systems. Studies on these and the related questions are in progress. 

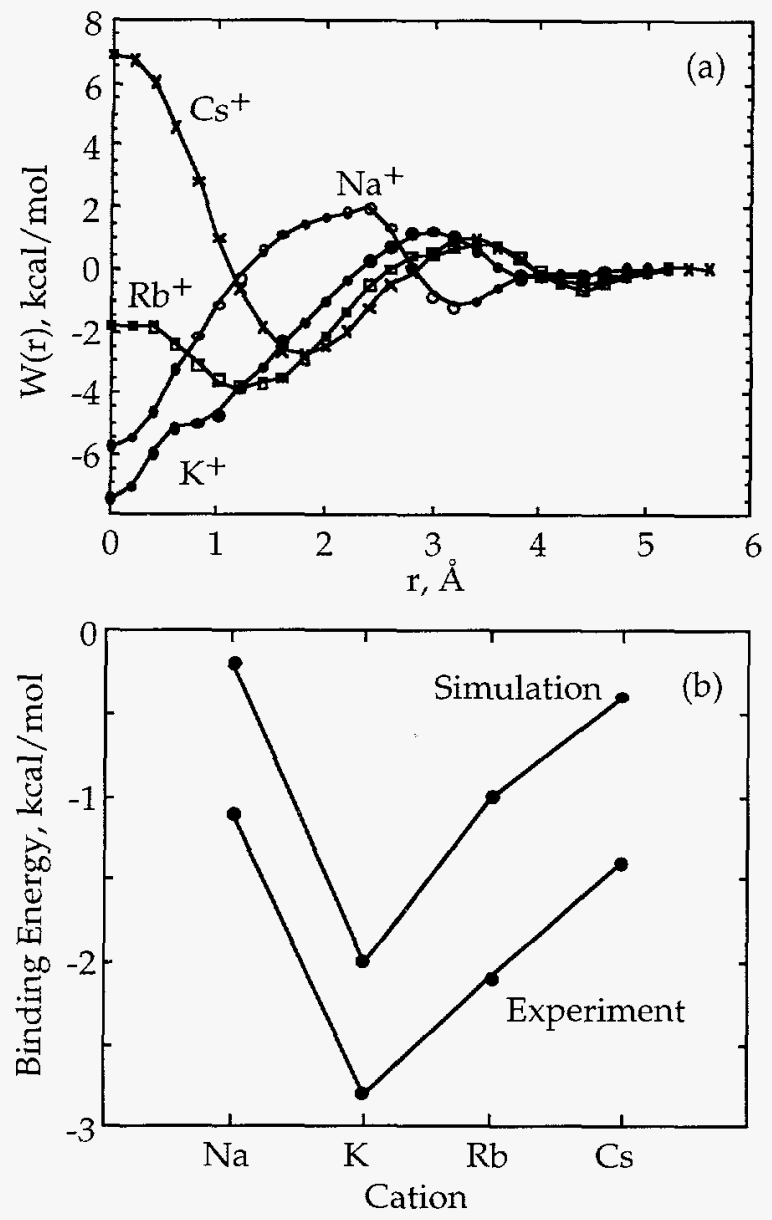

Figure 1.19. (a) The potential of mean force for the association of alkali cations to 18-crown- 6 ether in water near $300 \mathrm{~K}$. (b) Calculated binding energies of the cations to 18 -crown- 6 ether in water near $300 \mathrm{~K}$.

\section{Modeling Molecular Processes at Aqueous Interfaces}

The chemistry that occurs at interfaces controls many processes important to the transport of contaminants in the environment as well as to chemical aging in the tanks. Research on the chemistry at interfaces includes studies of aqueous interfaces with air (or vapor), other liquids (e.g., carbon tetrachloride), crystalline solids (e.g., mineral oxides, clays, found in soils), and glasses. The studies of air-vapor interfaces are important to understanding heterogeneous processes that occur in the atmosphere. The studies of liquidliquid interfaces are important to understanding the transport of molecules and ions between two immiscible liquids, which is relevant in a number of fields such as electrochemistry, separations, and the interaction of contaminated organic solvents with ground water. The studies of aqueous-mineral interfaces is a joint project with staff in the Solid State Theory and Modeling Group and is important to understanding the binding of contaminants to the soil. The studies of glass-water interfaces provides information on the dissolution and degradation of materials that are being proposed for long-term storage of nuclear wastes.

Investigation of the Liquid-Vapor Interface of Water via Molecular Dynamics (R. S. Taylor, ${ }^{(a)}$ L. X. Dang, and B. C. Garrett)

(a) AWU Postdoctoral Research Associate

Heterogeneous processes are important components of the earth's atmospheric system. Many of these processes involve reactions with water droplets and ices. Understanding how aerosols and small gaseous molecules such as $\mathrm{SO}_{2}$ interact with the interface of water may lead to a better overall understanding of processes such as the depletion/creation of ozone the formation of acid rain or the degradation of anthropogenic chemicals. In this work, we are primarily interested in those processes which occur at water's liquidvapor interface.

Molecular dynamics (MD) computer simulations can be used to gain insight into these processes. The accuracy of any MD simulation, however, is limited by the accuracy of the interaction potential functions being used. Potential functions are available which reproduce many of the properties of bulk water. ${ }^{87-89}$ To a large degree, the ability of these potentials to reproduce the properties of the water liquid-vapor interface remains as of yet undetermined. Although the SPC/E potential of Berendsen and coworkers ${ }^{87}$ does remarkably well at reproducing many of the bulk properties of water, at the interface between liquid water and its vapor, the environment of the individual water molecules is no longer bulk-like. As the interface is approached, each water molecule has fewer water molecules surrounding it. The effect that the environment has on the individual molecules can be seen by comparing the dipole moment of liquid water (2.6 Debye) where each water molecule is solvated on average by 
four other water molecules and that of a lone water molecule in the gas phase (1.8 Debye). Inasmuch as pair-wise potential functions such as the SPC/E model of water assume a constant dipole moment, it is not obvious that they will be able to predict the correct behavior of the water liquid/vapor interface. Thus, before embarking on modeling complex heterogeneous reactions involving the liquid-vapor interface of water, we must first determine to what extent we can reproduce the experimentally measurable properties of this interface.

The surface tension, or surface free energy, $(\gamma)$ is a measure of the degree of structure possessed by the interface or of the desire of the molecules to be solvated. Since the surface tension is in effect a two-dimensional free energy, monitoring the surface tension as a function of temperature also allows the surface entropy to be determined. 90 Using the theories of Kirkwood and Buff, 91,92 the surface tension of a SPC/E water can be determined in a MD simulation using the following equation:

$\gamma=\frac{1}{4 \mathrm{~A}}\left\langle\sum_{\mathrm{i}, \mathrm{j}} \sum_{\alpha, \beta}\left(\frac{\partial \mathrm{v}_{\alpha \beta}}{\partial \mathrm{r}_{\alpha \beta}}\right)\left(\frac{1}{\mathrm{r}_{\alpha \beta}}\right)\left(\overrightarrow{\mathrm{r}}_{\mathrm{ij}} \cdot \overrightarrow{\mathrm{r}}_{\alpha \beta}-3 \mathrm{z}_{\mathrm{ij}} \mathrm{z}_{\alpha \beta}\right)\right\rangle$

where $A$ is the area of the liquid-vapor interface, $V_{\alpha \beta}$ is the potential energy calculated between sites $\alpha$ and $\beta$ on molecules $i$ and $j, r_{\alpha \beta}$ and $z_{\alpha \beta}$ are the bond distance and the distance in the $z$ direction between sites $\alpha$ and $\beta$, and $r_{i j}$ and $z_{i j}$ are the corresponding quantities calculated between center of masses of molecules $i$ and $j$. The surface tension of SPC/E water (solid circles) calculated as a function of temperature is shown in Figure 1.20 along with the corresponding experimental data (open circles). The error bars are calculated from the standard deviation of at least five 50 ps trajectories. Given the simplicity with which the SPC/E potential treats the water molecule, the agreement between the simulations and the experimental data is quite good. The dashed line is a best linear fit to the simulation data. From the slope of this line, the surface entropy is found to be $0.230 \mathrm{dyn} / \mathrm{cmK}$ which is $39 \%$ larger than the value calculated for the experimental data of $0.165 \mathrm{dyn} / \mathrm{cmK}$. Additional work examining to what extent the SPC/E model can reproduce other properties such as the surface potential and orientation is presently under way.

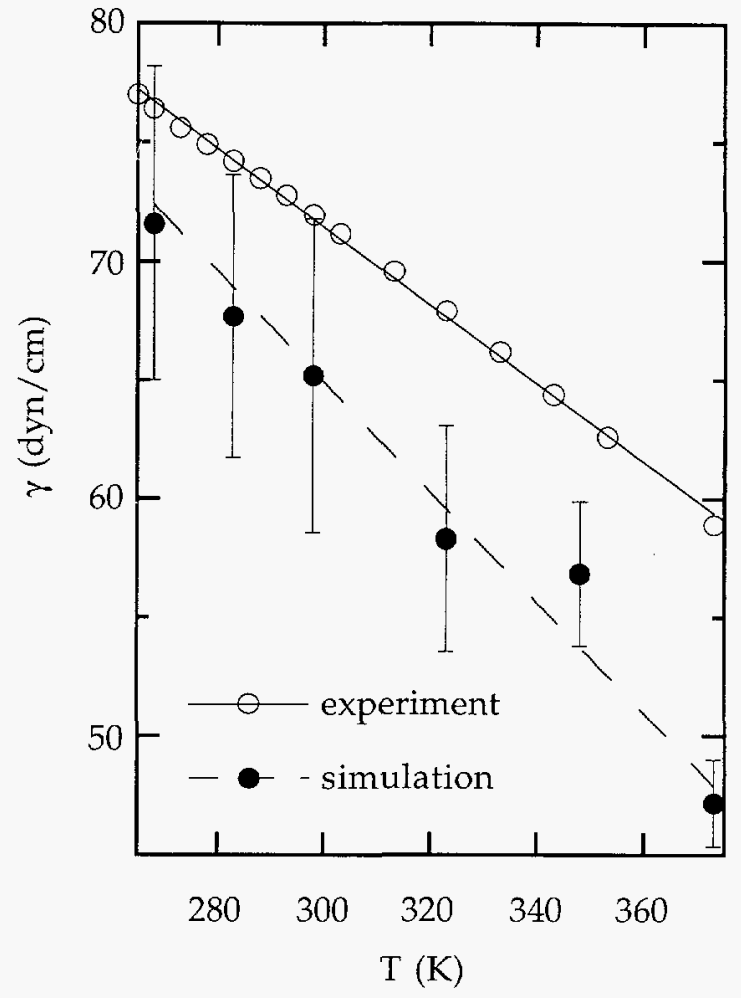

Figure 1.20. The surface tension of water plotted as a function of temperature. The solid line is a linear fit to the experimental data ${ }^{90}$ (open circles). The solid circles are obtained from MD simulations using the SPC/E potential model of water. The error bars on these points are obtained from the standard deviation of at least five 50 ps trajectories. The dashed line is the best linear fit to this data.

Solvation of the $\mathrm{Cs}^{+}$Ion at the Liquid-Vapor Interface of Water (L. X. Dang, R. S. Taylor, ${ }^{(a)}$ and B. C. Garrett)

(a) AWU Postdoctoral Research Associate

As part of our effort to achieve a theoretical understanding of interface phenomena at the molecular level and to develop suitable waterwater and ion-water potential models, we have initiated studies of the solvation of ions at the water-vapor interface. We began the study by computing the density profiles of the $\mathrm{Cs}^{+}$ion and of water molecules at the interface using polarizable potential models. ${ }^{55}$ We found the $\mathrm{Cs}^{+}$ion remained localized close to the middle of the interface (Figure 1.21) and its density profile is approximately symmetric with respect to the 
midplane of the interface. Figure 1.21 also illustrates the density profiles for the oxygen and hydrogen atoms. The densities for oxygen and hydrogen are similar and indicate no atomic layering. We have also begun to calculate the free energy profile to move the $\mathrm{Cs}^{+}$ion through the liquid-vapor interface. The research will continue to study the solvation at interface of ions that are important in the environment.

Molecular Dynamics Studies of Processes at Liquid-Liquid Interfaces (T.-M. Chang ${ }^{(a)}$ and L. $X$. Dang)

(a) AWU Postdoctoral Research Associate

We began our effort in this area by constructing interaction potential models for organic solvents such as $\mathrm{CCl}_{4}$ and $\mathrm{CHCl}_{3}$. Using molecular dynamics techniques, a rigid five-site polarizable $\mathrm{CCl}_{4}$ model has been developed. This model accurately reproduces thermodynamic and structural properties of liquid $\mathrm{CCl}_{4}$. In Figure 1.22, we show the atomic radial distribution functions for $\mathrm{C}-\mathrm{C}, \mathrm{C}-\mathrm{Cl}$, and $\mathrm{Cl}-\mathrm{Cl}$ with peak positions in good agreement with experiment. ${ }^{93}$ Future work will focus on the characterizations of the $\mathrm{CCl}_{4}$-water interface by examining its density profiles, hy-

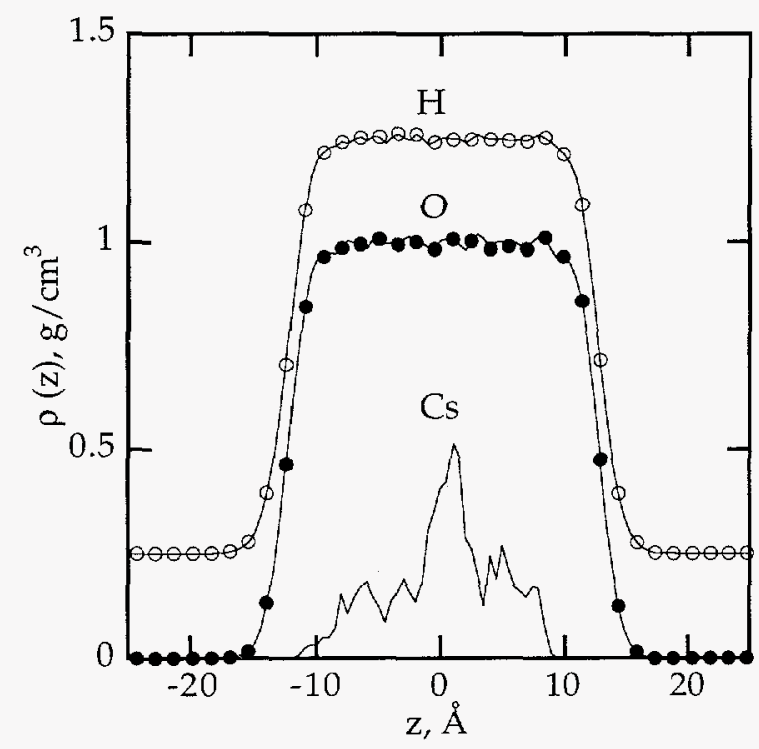

Figure 1.21. Average atomic density profiles obtained from molecular dynamics simulations at room temperature. Note that the hydrogen densities are shifted up. drogen bondings, and the molecular orientations, and transport mechanisms of ions and organic solute across the interface.

Physics and Chemistry of Structurally Simple Oxides (M. I. McCarthy, A. C. Hess, ${ }^{(a)}$ J. B. Nicholas, ${ }^{(b)}$ C. A. Scamehorn, ${ }^{(c)}$ M. R. ChaconTaylor, $^{(c)}$ M. Leslie, ${ }^{(d)}$ N. Harrison, ${ }^{(d)}$ and M. Towler $\left.^{(e)}\right)$

(a) Solid State Theory and Modeling Group

(b) High Performance Computational Chemistry Group

(c) AWU Postdoctoral Research Associate

(d) SERC Daresbury Laboratory, Daresbury, England

(e) Bristol University, England

The chemistry of naturally occurring and/or industrially important interfaces often involves dynamical processes of structurally and electronically complex materials. In particular, it is necessary to determine the properties of realistic surface sites (containing point defects, steps, kinks) to quantitatively predict surface chemistry. Magnesium oxide $(\mathrm{MgO})$ is a structurally simple cubic oxide material whose

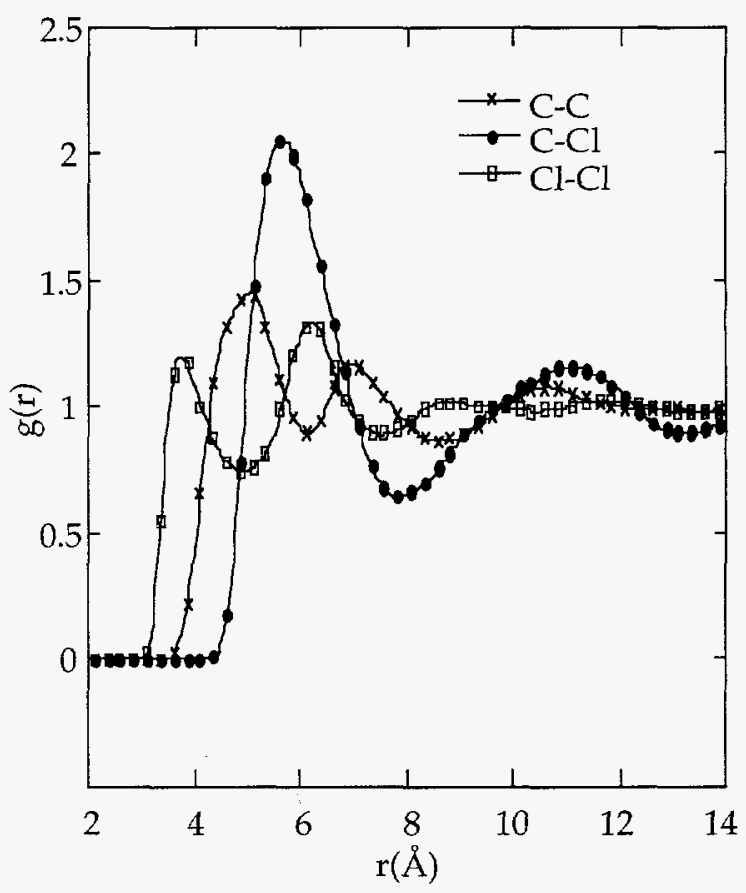

Figure 1.22. The atomic radial distribution functions of carbon-carbon, carbon-chloride, chloridechloride for liquid $\mathrm{CCl}_{4}$ at $298 \mathrm{~K}$. 
interfaces play an important role in subsurface geochemistry, catalysis and materials design. The bulk, surface, and interface properties of $\mathrm{MgO}$ are being studied to understand the behavior of $\mathrm{MgO}$ and to derive models that can be used to model processes involving more complex oxides. The composition (number of electrons) and structure (high symmetry) make this a 'computationally practical' system that can be studied in detail using $a b$ initio electronic structure theory. There is also a wealth of experimental data against which to benchmark our computational methods.

During FY 1994, research in this area probed several different problems. The electronic structure investigations of the $\mathrm{MgO} /$ water interface were extended to examine the effect of defect sites on the surface chemistry. ${ }^{94,95}$ Water is ever-present in the environment and can have a variety of interactions with exposed oxide surfaces, each of which will have a different effect on soil chemistry and on the transport of other chemicals through soils. Therefore, understanding the processes of water adsorption and chemical dissociation on oxide surfaces is fundamental to modeling subsurface transport phenomena. $A b$ initio periodic Hartree-Fock (PHF) theory (including correlation corrections) $)^{96-98}$ was used to study the chemistry of water on clean, defected, and strained $\mathrm{MgO}$ (001).

Structural defects were constructed containing both corner (3-coordinated) and edge (4-coordinated) sites. Isotropic distortions of the lattice were used to probe the properties of strained $\mathrm{MgO}$. The $a b$ initio energetics revealed that molecular water binds weakly $(\sim 10-15 \mathrm{kcal} / \mathrm{mol})$ to flat $\mathrm{MgO}(100)$ and more strongly (17-25 $\mathrm{kcal} / \mathrm{mol}$ ) to the defected sites. ${ }^{95}$ Mulliken populations show that a fundamental change occurs in the surface oxygen sites as the coordination number of these sites decreases while the magnesium sites remain relatively unaffected by changes in their local environment. The reduction of the Madelung field surrounding the oxygen sites results in the migration of small amounts of charge from the surface oxygens to oxygens residing in the bulk material leaving the oxygen defect sites capable of forming more strongly covalent bonds than sites on the perfect surface. Chemidissociation - resulting in surface hydroxylation - is found only to occur on surface defect sites and not on the equilibrium structure flat surface. However, chemidissociation becomes more energetically favorable as the flat lattice is isotropically distorted (becoming exothermic with $\sim 5-8 \%$ dilation of the lattice constant). This data indicates that the surface chemistry of $\mathrm{MgO}$ could be adjusted if stable thin films of the material could be prepared under strained conditions. The dramatically different chemistry on defected, clean, and strained surfaces illustrates, quantitatively, the importance of surface structure in regulating interfacial phenomena. Analytic expressions for the water/MgO (001) interaction potential were derived from the quantum mechanical data and are currently being used in classical simulations to examine the dynamical properties of the interface.

A new methodology was also developed to derive electrostatic models of oxide interfaces. These models are constructed from the energetics of an adsorbate - described by a multipolar expansion of the charge density - interacting with the quantum mechanical electric fields generated by the surface. The classical interaction energy between two systems (e.g., an adsorbate and a surface) can be described as multipolar expansion separated by the distance $(r)$ :

$$
\begin{array}{r}
\mathrm{V}_{\mathrm{ME}}(\overrightarrow{\mathrm{r}})=\mathrm{q}^{\mathrm{mol}} \phi^{\operatorname{surf}}(\overrightarrow{\mathrm{r}})-\overrightarrow{\mathrm{p}}^{\mathrm{mol}} \cdot \overrightarrow{\mathrm{E}^{\text {surf }}(\overrightarrow{\mathrm{r}})} \\
-\frac{1}{6} \mathrm{Q}_{\mathrm{ij}}^{\mathrm{mol}} \frac{\partial \mathrm{E}_{\mathrm{j}}^{\text {surf }}}{\partial \mathrm{r}_{\mathrm{i}}}(\overrightarrow{\mathrm{r}})+\cdots
\end{array}
$$

where $\mathrm{q}^{\mathrm{mol}}, \overrightarrow{\mathrm{p}}^{\mathrm{mol}}$ and $\mathrm{Q}_{\mathrm{ij}}^{\mathrm{mol}}$ are the charge, dipole moment, and quadrapole moment of the molecule, respectively, and $\phi^{\text {surf }}$ and $\vec{E}^{\text {surf }}$ are the electric potential and electric field generated by the surface at the center of mass of the molecule. The terms on the right-hand side of this expression represent the interaction of a charged species with the surface potential, a permanent dipole with the field, and a molecular quadrapole with the field gradient. The field generated by the surface and the moments of the adsorbate are computed quantum mechanically and the adsorbate-surface interaction is deduced from the classical electrostatic interaction (see equation above). This electrostatic model produces a reliable computationally 'trivial' method for examining the properties of a range of 
interfaces. The results have been validated by comparing the classical data to quantum mechanical calculations on several systems. Excellent agreement was found between the computed electrostatic energetics and the quantum mechanical values for $\mathrm{HCl}, \mathrm{H}_{2} \mathrm{O}$, and $\mathrm{NH}_{3}$ on $\mathrm{MgO}$ (see Figure 1.23). This approach provides us with a quantitative interpretation of the interfacial bonding interactions that allow us to predict the properties of more complex, low symmetry materials.

Quantum mechanical methods were also used to examine the bulk properties of $\mathrm{MgO}$ and mixed $\mathrm{MgO} / \mathrm{NiO}$ solids. Ab initio PHF theory was used to determine the elastic constants and selected phonon frequencies of bulk MgO. ${ }^{99}$ These were used to assess the accuracy of a posteriori correlation corrections to the PHF calculations and as a

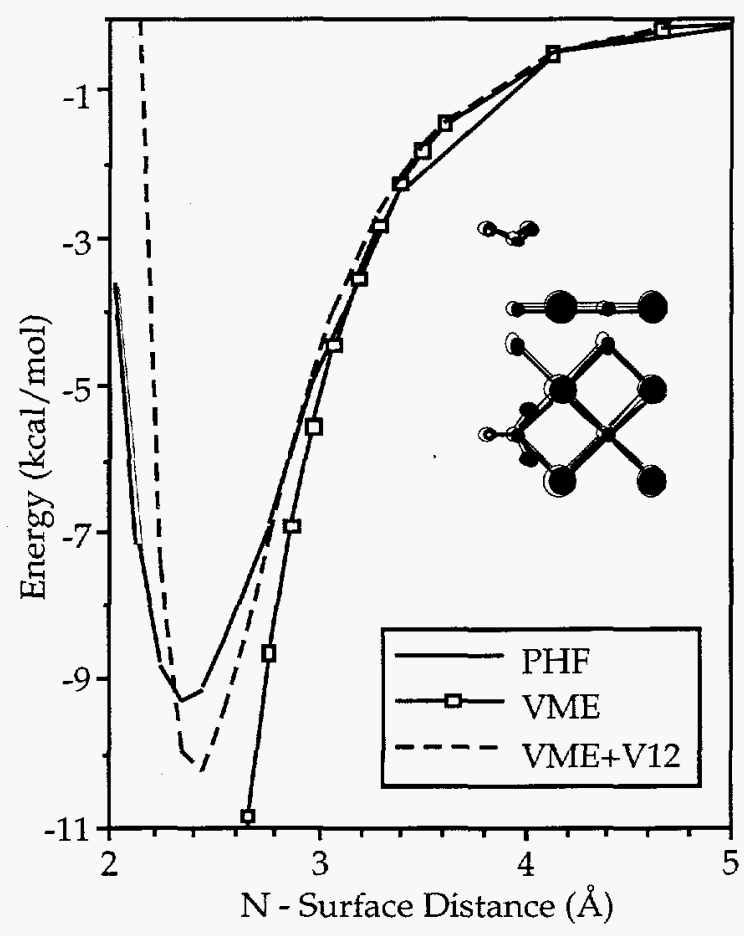

Figure 1.23. Comparison of $a b$ initio and electrostatic interaction energies for $\mathrm{NH}_{3}$ on $\mathrm{MgO}(001)$. $\mathrm{NH}_{3}$ is bound over the magnesium surface site with the nitrogen end pointing toward the surface. The repulsive potential (V12) is added to the purely electrostatic interaction potential (VME) to describe the classical electrostatic binding energetics. The surface oxygens are represented by bigger radii than the surface magnesiums. basis for parameterizing electrostatic shell models for the bulk material. Mixed MgO/NiO layered thin films possess unique structural and electronic properties that contribute to an enhanced chemical reactivity of the surfaces. These materials were examined theoretically to determine the electronic and geometrical properties of the composite-oxide thin films and to interpret anomalous $x$-ray photoelectron spectra reported for these materials. 100

$\mathrm{TiO}_{2}$ Surface Chemistry and Physics (M. I. McCarthy, M. A. Henderson, ${ }^{(a)}$ K. Briggman ${ }^{(b)}$ )

(a) Materials and Interfaces Program, EMSL

(b) Department of Chemistry, Northwestern University

Recent experimental work at PNNL, as part of the environmental restoration mission of the Hanford Site, has indicated that titanium dioxide $\left(\mathrm{TiO}_{2}\right.$, rutile) functions efficiently as an ion adsorber (e.g., in the extraction of $\mathrm{Sr}$ from waste streams) and as a thermal- and/or photo-catalyst in the destruction of organic and halogenated waste forms. A major effort is currently under way to determine which properties of the material control its interfacial chemistry. This work includes studies of the structural and electronic properties of the bulk and surfaces, the energetics of solution/ $/ \mathrm{TiO}_{2}$ interfaces and the mechanisms for electron-induced catalysis of organics.

The examination of the chemistry of water on $\mathrm{TiO}_{2}$ surfaces is being studied as part of a collaborative theoretical/experimental effort. Secondary ion mass spectroscopy (SIMS), temperature programmed desorption (TPD), electron energy loss spectroscopy, and low energy electron diffraction spectroscopy are being used to quantitatively determine the structure of clean and defected (110) and (100) surfaces, and the energetics and structure of the water $/ \mathrm{TiO}_{2}$ interface. Interpretation of these data are aided by comparison to $a b$ initio PHF calculations of several adsorbate/surface configurations. The initial aim of this study is to determine which surface sites (if any) result in the chemical decomposition of water and what are the resulting energetics of hydrated $\mathrm{TiO}_{2}$. The longterm goal of this work is to understand the behavior of solvated ions at $\mathrm{TiO}_{2}$ interfaces.

The surface of clean $\mathrm{TiO}_{2}(110)$ consists of alter 
nating troughs of titanium and oxygen atoms and ridges of oxygens. This surface is readily defected by the removal of selected bridging oxygens - exposing $\mathrm{Ti}^{3+}$ surface sites - when exposed to lowdensity electron beams. ${ }^{101}$ The chemistry of

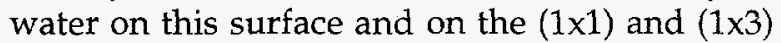
$\mathrm{TiO}_{2}$ (100) surfaces was probed by SIMS and TPD. ${ }^{102-105}$ The $(1 \times 3)(100)$ surface contains $\{11\}$ microfacets (Figure 1.24). Water readily binds to both of these faces (with unity sticking probability) and the desorption profiles are sensitive to the surface structure.

The $a b$ initio calculations of water on $\mathrm{TiO}_{2}(110)$ confirm that water is strongly bound (chemisorbed) with the water oxygen $\mathrm{O}_{\mathrm{W}}$ down over the trough titanium (binding energy 44 $\mathrm{kcal} / \mathrm{mol}$ ). Mulliken bond overlap analyses show that the formation of a positive bond overlap between $\mathrm{Ti}$ and $\mathrm{O}_{\mathrm{W}}$ is accompanied by a reduction in bond strength between the titanium and neighboring in-plane oxygens. This is indicative of the formation of a $\mathrm{Ti}-\mathrm{O}_{\mathrm{W}}$ chemical bond. An analogous configuration with the hydrogens approaching the surface $\left(\mathrm{O}_{\mathrm{W}}\right.$ "up" over $\mathrm{Ti}$ ) results in a purely repulsive adsorbate/surface interaction. The formation of a hydroxylated surface is computed to be the most energetically favorable interaction for water on $\mathrm{TiO}_{2}(110)$, with a binding energy of $73 \mathrm{kcal} / \mathrm{mol}$. The experimental data have not unambiguously determined if water is dissociated on the $\mathrm{TiO}_{2}$ (110) surfaces (or on the $\{110\}$ microfacets). The computed energetics indicate that the dissociative state is the most energetically favorable, but these data do not describe the potential energy surface along the dissociation pathway. Current studies are examining the energetics of the dissociation channel on this surface using both $a b$ initio quantum mechanics and electrostatic models.

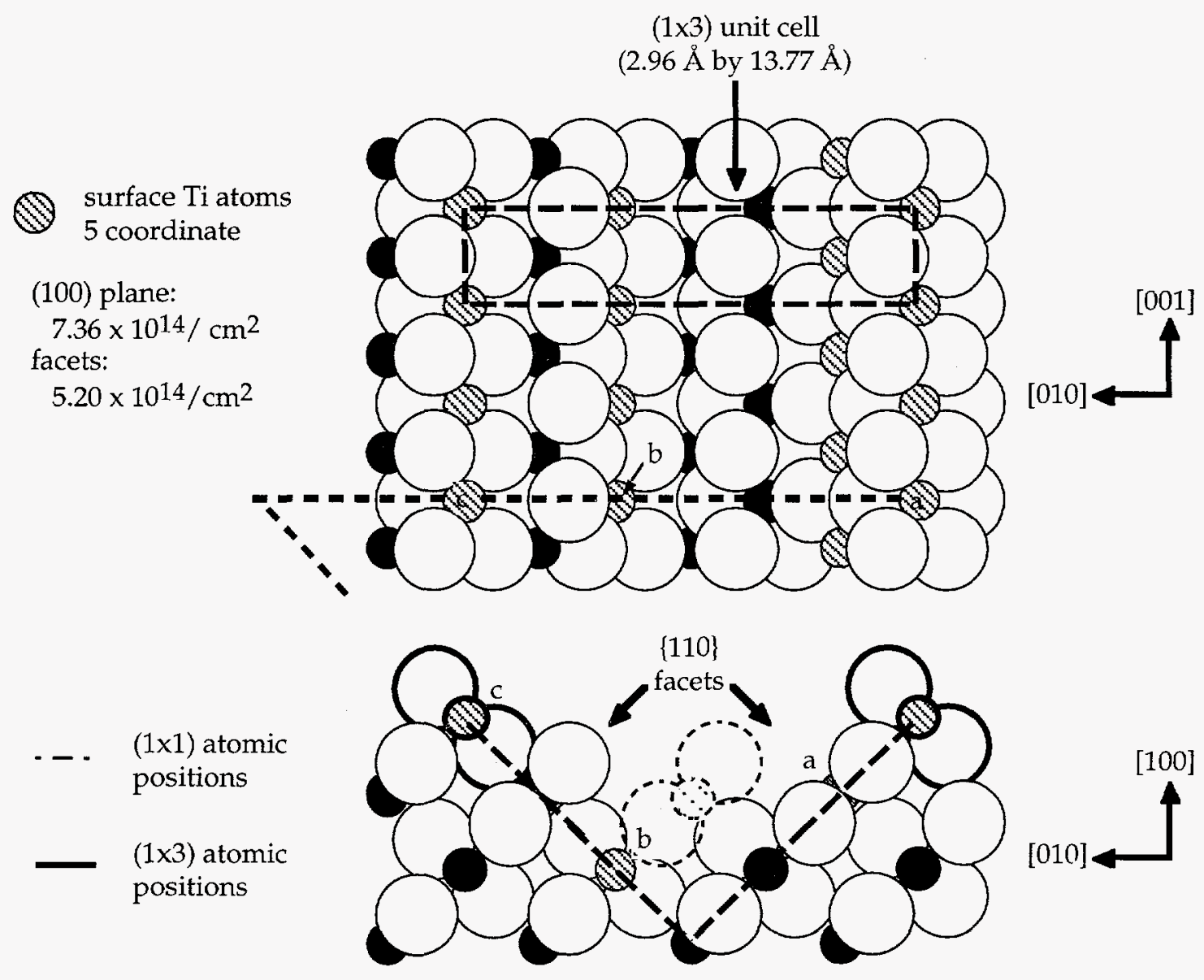

Figure 1.24. The unrelaxed microfaceted surface of TiO2 (100) (1x3). 
Laser Ablation of Molecular-Ionic Crystals (M. I. McCarthy, K. A. Peterson, W. P. Hess, ${ }^{(a)}$ T. M. Orlando, ${ }^{(a)}$ and R. A. Bradley ${ }^{(b)}$ )

(a) Chemical Structure and Dynamics Program, EMSL

(b) AWU Postdoctoral Research Associate in Chemical Structure and Dynamics Program, EMSL

Laser ablation combined with mass spectrometry (LAMS) is being developed as a diagnostic for analysis of atomic and molecular species in mixed hazardous wastes. ${ }^{106}$ Using this approach, analysis of complex, multicomponent mixtures can be performed rapidly using very little sample. Reduced sample size is highly desirable for the analysis of many hazardous wastes because it minimizes the secondary waste generated from the analytic procedures. Specifically, the LAMS technique is being applied to analyze mixed wastes extracted from the underground storage tanks at the Hanford Site. A major component of these tanks is sodium nitrate $\left(\mathrm{NaNO}_{3}\right)$, hence understanding the ablation mechanisms of this material is essential to analyzing these wastes. ${ }^{107}$ A joint theoretical/experimental effort at PNNL is studying the mechanisms involved in the laser ablation of $\mathrm{NaNO}_{3}$ to deduce the optimal conditions for applying laser ablation as a "chemical," analytical diagnostic. Knowledge of ablation mechanisms is needed to determine the correlation between the ablation products and the original sample composition. Under certain conditions (e.g., high power density) the final products may be heavily influenced by the chemistry occurring in the laser induced ablation plasma near the surface. A detailed description of the photochemical mechanisms is less crucial when LAMS is used solely as a tool for elemental analysis where only the percent composition, and not the molecular speciation, of a material is sought. However, it is critically important to understand the chemistry of the ablation process if LAMS is to be used as a diagnostic tool for determining the chemical composition and structure of the original material.

Sodium nitrate is a wide-band-gap, insulating material that forms a molecular ionic crystal with a hexagonal unit cell of $D_{3 d}$ symmetry. Molecular-ionic crystals are unique materials because they possess strong 'molecular' absorption in ultraviolet regions that are readily accessed by laboratory experiments. The photochemistry of the molecular ion chromophore, in turn, dictates the final product distributions. The laser/solid interactions following resonant excitation of single-crystal sodium nitrate are probed experimentally by measuring the translational, rotational, and vibrational energy distributions of desorbed NO. ${ }^{108}$ The $\mathrm{NaNO}_{3}$ crystals are excited using 5-ns pulses of $213 \mathrm{~nm}$ and $266 \mathrm{~nm}$ light incident on the sample at $40^{\circ}$ to the crystal face. Neutral NO fragments are probed using $1+1$ multiphoton ionization (MPI) following ablation. The positive ions produced via MPI are detected using a time-of-flight (TOF) mass spectrometer. The neutral NO desorption yield is quantitatively different for resonant excitation of the $\pi^{*} \leftarrow \pi_{2}$ band at $213 \mathrm{~nm}$ as compared to nonresonant excitation at $266 \mathrm{~nm}$. For freshly cleaved samples, the neutral NO yield for 213-nm excitation is approximately 1000 times greater than for 266$\mathrm{nm}$ excitation, hence it is clear that resonant excitation leads to a significant enhancement of the NO yield due to a photochemical reaction. Examination of the power dependence of the ablation process indicates that all NO production is a result of single photon absorption at $213 \mathrm{~nm}$.

Photoexcitation of the $\pi^{*} \leftarrow \pi_{2}$ band in $\mathrm{NaNO}_{3}$ creates excited-state nitrate anion $\mathrm{NO}_{3}^{-*}$ (a Frenkel exciton) both at the surface and in the bulk crystal. Excitons formed in the bulk material can hop to sites at or near the surface. The surface dynamics of photoexcited $\mathrm{NaNO}_{3}$ results from the dissociation of the $\mathrm{NO}_{3}^{-*}$ exciton at or near the surface. Since this exciton presumably self traps at the vacuum surface interface, the subsequent dissociation mechanisms can be described in terms of the decay of a surface-bound negative ion excited state. Hence, the ablation process is actually probing the photochemistry of the $\mathrm{NaNO}_{3}$ gas-solid interface. At or near the surface layer, direct photodissociation can occur, leading to desorption of NO, by the proposed mechanism:

$$
\begin{aligned}
\mathrm{NO}_{3(\mathrm{~s})}^{-}+\mathrm{hv} & \rightarrow \mathrm{NO}_{3}^{-*}(\mathrm{~s}) \\
& \rightarrow \mathrm{NO}_{(\mathrm{g})}+\mathrm{O}_{2(\mathrm{~s})}^{-}
\end{aligned}
$$

Here (s) indicates surface or near surface sites, and $(\mathrm{g})$ indicates escaped gas phase species. According to this mechanism, the $\mathrm{O}_{2}^{-}$product remains trapped on the surface in the defect site created by the decomposition of the nitrate ion.

The ablation mechanisms have been probed using $a b$ initio quantum mechanical methods that examine the effects of the crystalline environment 
on the spectroscopy and chemistry of the ions. A mechanism for the ablation process has been proposed based on theoretical investigations of the ion excitation spectra and the electronic properties of the ground state of pure and defected sodium nitrate crystals. In the gas phase, the excited $\mathrm{NO}_{3}^{-*}$ anion autodetaches to form the neutral excited state which dissociates to form neutral products. Stabilization of the anion states in the crystal (below the neutral channels) removes the neutral channels in the solid. This implies that photodissociation in the crystal proceeds directly from the excited nitrate ion to the formation of neutral gas-phase products and surface trapped negative ion photoproducts. The solidstate mechanism is qualitatively different from the gas phase mechanisms that proceed through the neutral $\mathrm{NO}_{3}$ intermediate to form only neutral products (see Figure 1.25). Periodic HartreeFock calculations of the bulk material revealed that there are negligible shifts in the ground state electronic structure when the crystal is cleaved along the 1014 plane. However, significant changes in the character of the ground state does

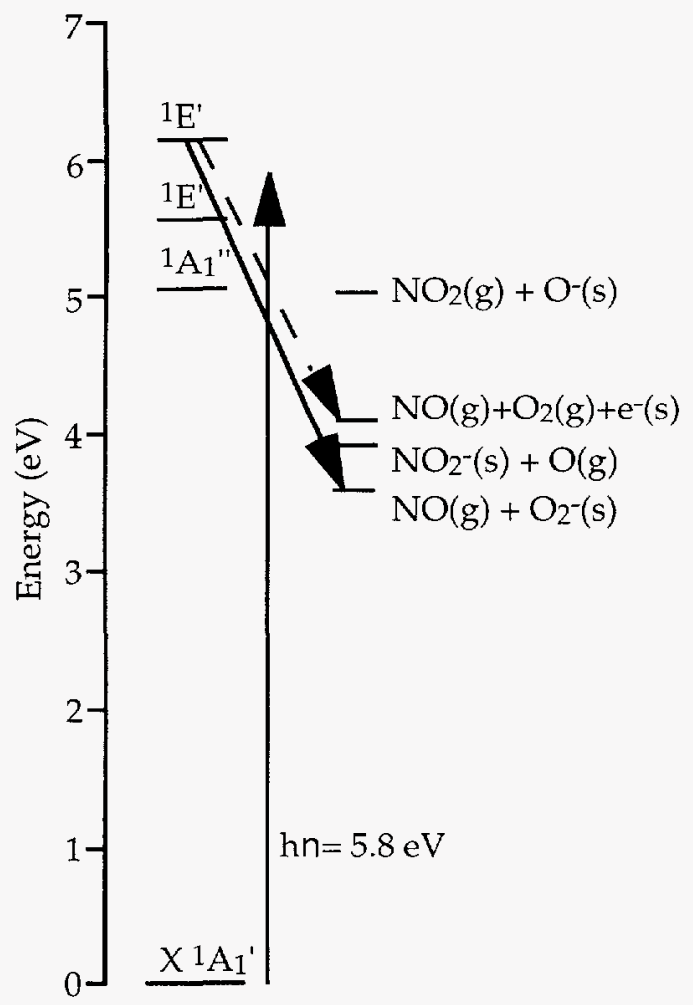

Figure 1.25. Surface ablation mechanism for $\mathrm{NaNO}_{3}$. occur when defects are present on the surface (e.g., outer oxygens are removed to produce surface nitrite groups). Ongoing theoretical and experimental studies are probing the sensitivity of the ablation product yield and distributions to surface and bulk defects.

Heterogeneous Interface Chemistry of Ices (M. I. McCarthy, R. D. Poshusta, ${ }^{(a)}$ and S. George ${ }^{(b)}$ )

(a) Department of Chemistry, Washington State University

(b) Department of Chemistry, University of Colorado

Water and mixed ices play key roles in regulating the chemistry of the atmosphere. Studies of hydrogen bonding, proton transfer, and interface structure and chemistry in ices can also be used to understand similar processes in more complex environments (e.g., Cryocell in situ barrier design, mixed hazardous wastes, biological systems). $A b$ initio PHF calculations on the bulk and surfaces of nitric acid mono- and tri-hydrates (the major constituent of type II polar stratospheric clouds) revealed which sites on these surfaces are expected to be chemically accessible and reactive to adsorbate molecules. 109 The nature (structure) of ice and mixed ice interfaces is presently a topic of considerable debate among experimentalists, atmospheric modelers, and theoretical chemists. If the ice-water interface is less structured (e.g., more liquid-like), ion solvation effects are expected to dominate the chemistry of adsorbed species. More crystalline surfaces, by contrast, would be governed by the gas-solid interfacial properties.

The inherent disordering of the protons in ice makes this a difficult system to characterize both experimentally and theoretically. Ice Ih is the most common phase of ice at low pressures and is characterized by a disordering of the protons in an orthorhombic lattice. The oxygen atom positions, measured by $x$-ray and neutron scattering, are tetrahedrally coordinated and the protons are statistically distributed between oxygens according to the Bernal-Fowler-Pauling (BFP) rules. ${ }^{110,111}$ Each oxygen is covalently bonded to two protons $(\mathrm{ROH}=1.01 \AA$ ) and hydrogen bonded to two others ( $\mathrm{RO}-\mathrm{H}=1.75 \AA$ ). The $\mathrm{OH}$ bond distance in ice is substantially longer than the corresponding gas phase value $(0.95 \AA)$ and the $\mathrm{HOH}$ angle is larger $\left(109^{\circ}\right.$ in ice and $104^{\circ}$ in gas phase water). A partially ordered structure 
has been prepared by freezing a dilute $(\sim 0.1 \mathrm{M})$ aqueous $\mathrm{KOH}$ solution. 112 The doped ice contains ordered domains $(\sim 70 \%)$ and disordered regions $(\sim 30 \%)$. Neutron diffraction measurements on the doped ice indicate that the ordered domains are composed of a ferroelectric structure. ${ }^{113}$ An earlier theoretical study computed the relative stabilities of a range of ordered ice structures to determine the energetically favorable geometries. ${ }^{114}$

At absolute zero the protons in ice exist in the lowest energy ordered structure; at higher temperatures the distribution among locally ordered structures is dictated by statistical mechanics. The relative energetics of the ordered proton structures is therefore important for determining the bulk properties of ice. A systematic method for deriving the crystallographically allowed ordered ice structures that are consistent with the disordered proton model was devised theoretically. The aim of this work was to systematically classify the possible ordered structures that can be constructed from 4- and 8-water disordered lattices. $A b$ initio PHF calculations on the fourwater structures indicated that these structures are essentially energetically identical (within 1 $\mathrm{kcal} / \mathrm{mol}$ ). The calculated cohesive binding energy, using correlation-corrected PHF theory, is $\sim 13 \mathrm{kcal} / \mathrm{mol}$. This is in excellent agreement with the measured heat of vaporization 13.4 $\mathrm{kcal} / \mathrm{mol}$.

A model of the surface of crystalline hexagonal ice can be constructed based on the 4-water models described above. The structure and reactivity of crystalline ice surfaces are currently being investigated using PHF theory in an effort to understand the interfacial chemistry in hydrogenbonded systems. The initial investigations are probing the energetics of adsorption of $\mathrm{HCl}$ onto ice $(1 \mathrm{~h})$. The goal of this work is to characterize the nature of the $\mathrm{HCl}$ /ice interface and to determine the mechanisms for subsequent surface reactions.
Physics of Polymer Interfaces (M. I. McCarthy, R. A. Kendall, ${ }^{(a)}$ K. deDing, ${ }^{(b)}$ D. A. Dixon, ${ }^{(c)}$ and J. Glas $(c)$

(a) High Performance Computational Chemistry Group

(b) AWU Postdoctoral Research Associate, Department of Chemistry, University of Washington

(c) DuPont Central Research and Development, Wilmington, Delaware

A joint project (Cooperative Research and Development Agreement, CRADA) with DuPont has been established to understand the bulk, fluid, and interface properties of fluorinated polymers (neat and substituted tetrafluoroethylenes). The goal of the project is to predict the optimal chemical composition that would allow for the production of thin polymer films on oxide surfaces. Three aspects of this problem are being addressed. First, the dependence of the solid properties (e.g., stress/strain, electrical insulation) on chemical composition is being studied using molecular and periodic quantum mechanical methods. Second, the structural (radius of gyration, end-to-end distance) characteristics of the corresponding polymer melts are probed using statistical mechanical methods. Understanding the melt is essential because the industrial manufacture of thin film coatings requires processing the polymer in a liquidlike'phase. The third component of this work will examine the properties of polymer/oxide interfaces.

A fundamental area of research in polymer physics involves determining the correlation between atomic properties of a monomeric unit and the macroscopic observables of the corresponding polymer. In many cases small perturbations in the composition of a polymer (e.g., low concentration site-defects) can have an extensive impact on the properties of the material. This CRADA project is investigating these phenomena by probing the effects of chemical composition on electronic, mechanical, and melt properties of polytetrafluoroethylene (PTFE) and substituted PTFE compounds (see Figure 1.26). PTFE is a high molecular weight ( $\sim 30-50$ million) material with unique electrical and mechanical properties. ${ }^{115-}$ 118 In its technologically important phases, the PTFE chains are helical. The corresponding hydrogenated material, polyethylene (PE), does not exhibit a helical structure and has vastly different physical traits. An integrated theoretical 


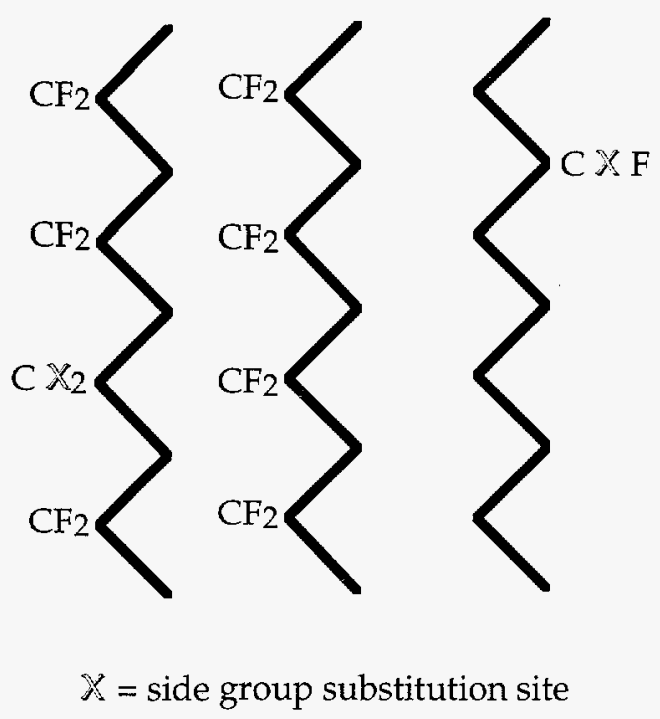

Figure 1.26. Substituted polytetrafluoroethylene.

approach based on quantum mechanical electronic structure theory and statistical mechanics is being used to investigate the connection between chemical composition and the bulk properties of these materials.

Ab initio electronic structure theory, based on periodic and correlated Hartree-Fock methods and density functional theory, are being used to map out the potential energy surface for PE, PTFE, and substituted PTFE; these methods are implemented in the codes NWChem ${ }^{119}$ and CRYSTAL. 96,97 These calculations are probing the nature of the helical structure in PTFE; the bulk, young, and shear moduli of the crystalline polymers; the effects of long-range order on the stress-strain properties of PTFE as a function of dimensionality (one-dimensional single strand, two-dimensional thin film, three-dimensional crystalline solid); and the mechanisms for uncoiling the PTFE helix. Complementary studies using statistical mechanics methods ${ }^{120,121}$ are studying the properties of the melt phases of these materials; these include determining the structure factors, melt viscosity, glass transition temperature $\left(\mathrm{T}_{\mathrm{g}}\right)$, melt temperature $\left(\mathrm{T}_{\mathrm{m}}\right)$, and crystallization mechanisms. Models are being developed that allow us to correlate features in the computed potential energy surfaces with specific melt or bulk properties.
A Semiempirical Molecular Dynamics Methodology for Simulating Covalently Bonded Materials: $\mathrm{Si}, \mathrm{C}, \mathrm{SiC}$, and Se (L. R. Corrales, A. Chen, ${ }^{(a)}$ and S. M. Slate ${ }^{(b)}$ )

(a) AWU Postdoctoral Research Associate

(b) AWU Undergraduate Research Associate

Electronic degrees of freedom in simulation model potentials play an important role when simulating the surface, melt, cluster, and amorphous solid states of covalently bonded materials. The influence of the electronic coordinates on the underlying geometric structure can be appreciated by the following examples. When silicon or selenium melt from their crystalline or amorphous solid states, large fluctuations in the local structure lead to a localized/delocalized electronic state transition. In surfaces and clusters the electronic configuration invokes a structural reconstruction to compensate for dangling bonds and bond strain. Topological disordering occurs in the melt during diffusion due to bond forming and bond breaking events, where the disorder persists upon rapid cooling which leads directly to a glass state. The localized/delocalized transition, the reconstruction of local structure, and the changes in bond connectivity are all associated with a change in both the electronic and ionic structure. Therefore, modeling these materials with a single model potential requires a method in which the electronic coordinates are coupled with the ionic interactions.

In general, first principle calculations can provide a proper description capable of capturing the coupling between electronic states and the geometry. Where as simulation model potentials based on DFT, Hartree-Fock theory, and tightbinding methods provide excellent descriptions of the electronic states, they remain too computationally intensive for use in large-scale MD simulations. Classical methods that use empirical potentials without an adequate description of the electronic states insufficiently describe structural changes associated with the electronic fluctuations. We are interested in using a model capable of large-scale simulations to examine dynamical and geometric structural properties of surfaces, interfaces, and bulk liquid and amorphous states of covalent materials. Thus we look toward a classical simulation approach that incorporates a first principles, albeit approximate, description of the electronic states. In this work, 
we use a recently introduced semi-empirical methodology and modify it to model and simulate silicon, carbon, silicon-carbide, and selenium.

The electronic coordinates of this model potential are the hybridization and rotational states of the valence orbitals on each atom. The semiempirical and classical parameters are fit to the gas phase dimer, the lowest energy bulk crystal lattice constant and cohesive energy. The relationship between specific bonding and nonbonding interactions with the local and global fluctuations can be obtained with this approach. The model can be used to provide insight into the intimate relationship between the electronic states and the geometric structure of bulk melts, amorphous solids, and surface states.

We have used optimization techniques to obtain properties of the bulk diamond crystal and the (100) reconstructed surfaces of $\mathrm{Si}, \mathrm{C}$, and $\mathrm{SiC}$. In particular, the Newton-Raphson method is used when the analytic second derivatives are easily calculated and otherwise the zero velocity Verlet algorithm is used. The energy derivatives can be determined analytically for the simpler case of $\sigma$ bonding, for which we obtain the higher order derivatives easily. We make use of the HellmanFeynman theorem to determine the first derivatives when $\pi$ bonds are present.

This potential satisfactorily reproduces the low temperature properties of the diamond phases of silicon and carbon. Single defect energies of the diamond crystal were also computed and are in agreement with other calculational methods. We have also carried out a detailed examination of the $\mathrm{Si}(100) 2 \times 1$ reconstructed surface and the Si adatom binding energetics ${ }^{122}$ on this surface. We find that the structure of the reconstructed surface as well as the structure and energetics of the adatom binding sites are strongly dependent on the presence of $\pi$ bonds.

This method accounts for near neighbor charge transfer effects in mixtures, like $\mathrm{SiC}$, that further stabilize the covalent bond. We have determined the net effect of including and excluding charge transfer in silicon carbide, and compared our results with those from first principle and classical potential calculations which do not include charge transfer. Our results are in agreement with the respective approaches and show the importance of including charge transfer.

Unlike empirical calculations, no additional parameters are required since we are able to use the parameterization of carbon and silicon to model a silicon carbide system without changes. We must, however, take into consideration charge transfer effects when using the model in MD simulations where bond exchange occurs as in melts and in the reconstruction of surfaces.

Distinct trends are found between classical and $a b$ initio calculations that are similar to the trends found when we exclude and include charge transfer in this work. The distinct differences in the energies, lattice constants, and bulk modulus show that the polarization is important in the bonding interactions. Similar trends are also observed in the defect energies where higher defect energies are observed in the polarized potentials.

We have cast the methodology into a molecular dynamics simulation method and used it to obtain properties of the low temperature crystals, the melt, and amorphous solid states of $\mathrm{Si}$ and $\mathrm{Se}$. We have obtained the pair distribution function, , distribution of bond angles and hybridization, and the self diffusion as a function of temperature for all states. The overall results are encouraging and validate the use of this potential to simulate these types of systems.

We have shown that a semiempirical potential cast in a classical simulation methodology is capable of grasping the essential qualitative and quantitative features of the influence of electronic coordinates on the geometric structure. An additional advantage of expressing the semiempirical contribution in terms of a pairwise potential is that the forces on a given atom are quickly and easily calculated, making this an extremely useful method for large-scale and long-time simulation studies.

\section{References}

T. H. Dunning, Jr., J. Chem. Phys. 90, 1007 (1989).
2 R. A. Kendall, T. H. Dunning Jr., and R. J. Harrison, J.
Chem. Phys. 96, 6796 (1992).
3 D. E. Woon and T. H. Dunning Jr., J. Chem. Phys. 98,
1358 (1993).
D. E. Woon and T. H. Dunning Jr., J. Chem. Phys. 99,
1914 (1993).


5 K. A. Peterson, R. A. Kendall, and T. H. Dunning Jr., J. Chem. Phys. 99, 1930 (1993).

6 K. A. Peterson, R. A. Kendall, and T. H. Dunning Jr., J. Chem. Phys. 99, 9790 (1993).

7 D. E. Woon and T. H. Dunning Jr., J. Chem. Phys. 101, 8877 (1994).

8 K. P. Huber and G. Herzberg, Molecular Spectra and Molecular Structure IV. Constants of Diatomic Molecules (Van Nostrand, Princeton, 1979).

9 C. W. Bauschlicher and S. R. Langhoff, J. Chem. Phys. 87, 2919 (1987).

10 R. S. Urdahl, Y. Bao, and W. M. Jackson, Chem. Phys. Lett. 178, 425 (1991).

11 A. M. Smith, S. L. Coy, and W. Klemperer, J. Mol. Spectrosc. 134, 134 (1989).

12 S. Glasstone, K. J. Laidler, and $\mathrm{H}$. Eyring, The Theory of Rate Processes (McGraw-Hill, New York, 1941).

13 D. G. Truhlar and B. C. Garrett, Acc. Chem. Res. 13, 440 (1980).

14 D. G. Truhlar and B. C. Garrett, Ann. Rev. Phys. Chem. 35, 159 (1984).

15 D. G. Truhlar, A. D. Isaacson, and B. C. Garrett, in Theory of Chemical Reaction Dynamics, Vol. IV, edited by M. Baer (CRC Press, Boca Raton, Florida, 1985), pp. 65.

16 A. D. Isaacson, D. G. Truhlar, S. N. Rai, R. Steckler, G. C. Hancock, B. C. Garrett, and M. J. Redmon, Comp. Phys. Comm. 47, 91 (1987).

17 D. H. Lu, T. N. Truong, V. S. Melissas, G. C. Lynch, Y. P. Liu, B. C. Garrett, R. Steckler, A. D. Isaacson, S. N. Rai, G. C. Hancock, I. G. Lauderdale, T. Joseph, and D. G. Truhlar, Comp. Phys. Comm. 71, 235 (1992).

G. A. Voth, D. Chandler, and W. H. Miller, J. Chem. Phys. 91, 7749 (1989).

19 M. Messina, G. K. Schenter, and B. C. Garrett, J. Chem. Phys. 98, 8525 (1993).

20 G. K. Schenter, M. Messina, and B. C. Garrett, J. Chem. Phys. 99, 1674 (1993).

21 G. A. Voth, J. Phys. Chem. 97, 8365 (1993).

22 B. C. Garrett and G. K. Schenter, Int. Rev. Phys. Chem. 13, 263 (1994).

23 R. P. Feynman and A. R. Hibbs, Quantum Mechanics and Path Integrals (McGraw-Hill, New York, 1965).

24 R. P. Feynman, Statistical Mechanics (Addison-Wesley, Reading, Massachuttes, 1972).

25 D. G. Truhlar, Y.-P. Liu, G. K. Schenter, and B. C. Garrett, J. Chem. Phys. 98, 8396 (1994).

26 B. C. Garrett and G. K. Schenter, in Structure and Reactivity in Aqueous Solution, Vol. 568, edited by C. J. Cramer and D. G. Truhlar (American Chemical Society, Washington, D.C., 1994), pp. 122.

27 E. Roduner and D. M. Bartels, Ber. Bunsenges. Phys. Chem. 96, 1037 (1992).

28 E. Roduner, P. W. F. Louwrier, G. A. Brinkman, D. M. Garner, I. D. Reid, D. J. Arseneau, M. Senba, and D. G. Gleming, Ber. Bunsenges. Phys. Chem. 94, 1224 (1990).

29 J. M. Nicovich and A. R. Ravishankara, J. Phys. Chem. 88, 2534 (1984).

30 G. Mills, H. Jonsson, and G. K. Schenter, Surf. Sci. 324, 305 (1995).
G. K. Schenter, G. Mills, and H. Jonsson, J. Chem. Phys. 101, 8964 (1994).

S. K. Reese, S. C. Tucker, and G. K. Schenter, J. Chem. Phys. 102, 104 (1995).

39 S. S. Xantheas and T. H. Dunning Jr., I. Chem. Phys. 98, 8037 (1993).

40 S. S. Xantheas and T. H. Dunning Jr., J. Chem. Phys. 99, 8774 (1993).

41 O. Mó and M. Yáñez, J. Chem. Phys. 97, 6628 (1992).

42 D. Hankins, J. W. Moskowitz, and F. H. Stillinger, J. Chem. Phys. 53, 4544 (1970).

43 S. F. Boys and F. Bernardi, Mol. Phys. 19, 553 (1970).

44 S. S. Xantheas, J. Chem. Phys. 100, 7523 (1994).

45 J. C. White and E. R. Davidson, J. Chem. Phys. 93, 8029 (1990).

46 M. J. Frisch, G. W. Trucks, M. Head-Gordon, P. M. W. Gill, M. W. Wong, J. B. Foresman, B. G. Johnson, H. B. Schlegel, M. A. Robb, E. S. Replogle, R. Gomperts, J. L. Andres, K. Raghavachari, J. S. Binkley, C. Gonzalez, R. L. Martin, D. J. Fox, D. J. Defrees, J. Baker, J. J. P. Steward, and J. A. Pople, , Revision C ed. (Gaussian, Inc., Pittsburgh, Pennsylvania, 1992).

47 J. A. Odutola and T. R. Dyke, J. Chem. Phys. 72, 5062 (1980).

48 T. R. Dyke, K. M. Mack, and J. S. Muetner, J. Chem. Phys. 66, 498 (1977).

49 L. A. Curtiss, D. J. Frurip, and M. Blander, J. Chem. Phys. 71, 2703 (1979).

50 J. Reimers, R. Watts, and M. Klein, Chem. Phys. 64, 95 (1982).

51 S. Deshmukh, J. D. Myers, S. S. Xantheas, and W. P. Hess, J. Phys. Chem. 98, 12535 (1994).

52 S. S. Xantheas and T. H. Dunning Jr., J. Phys. Chem. 98, 13489 (1994).

53 L. X. Dang and D. E. Smith, J. Chem. Phys. 99, 6950 (1992).

54 B. J. Berne and D. Thirumalai, Ann. Rev. Phys. Chem. 37, 401 (1986).

55 L. X. Dang, J. Chem. Phys. 97, 1919 (1992).

56

57

D. Feller, J. Chem. Phys. 96, 104 (1992).

M. Feyereisen, G. Fitzgerald, and A. Komornicki, Chem. Phsy. Lett. 208, 359 (1993).

58 J. P. Richard, Adv. Carbocation Chem. 1, 121 (1989).

59 D. E. Smith and L. X. Dang, Chem. Phys. 221, 209 (1994).

60 G. Fleissner, A. Hallbrucker, and E. Mayer, J. Phys. Chem. 97, 4806 (1993).

61 W. L. Jorgensen and D. L. Severance, J. Am. Chem. Soc. 112,4768 (1990). 
D. J. Tobins and I. C. L. Brooks, Chem. Phys. Lett. 142, 472 (1987).

63 C. J. Pederson, J. Am. Chem. Soc. 89, 7017 (1967).

64 A. R. Katritzky, N. Malhotra, R. Ramanathan, J. R. C Kemerait, J. A. Zimmerman, and J. R. Eyler, Rapid Com. Mass. Spect. 6, 25 (1992).

D. W. Rebertus, B. J. Berne, and D. Chandler, J. Chem. Phys. 70, 3395 (1979). 11, $700(1990)$. . Gao and X. Xia, Science 258, 631 (1992).

M. A. Thompson, Argus 3.0, (Pacific Northwest National Laboratory, Richland, Washington, 1994).

M. A. Thompson, E. D. Glendening, and D. Feller, J. Phys. Chem. 98, 10465 (1994).

70 R. L. Jaffe, G. D. Smith, and D. Y. Yoon, J. Phys. Chem. 97, 12745 (1993).

71 G. D. Smith, R. L. Jaffee, and D. Y. Yoon, J. Phys. Chem. 97, 12752 (1993). J. Deisenhofer and H. Michel, Science 245, 1463 (1989). S. J. Weiner, P. A. Kollman, D. A. Case, U. C. Singh, C. Ghio, G. Alagona, S. Profeta, and P. J. Weiner, J. Am. Chem. Soc. 106, 766 (1984). J. Applequist, J. Am. Chem. Soc. 94, 2952 (1972).

M. A. Thompson, M. C. Zerner, and J. Fajer, J. Phys. Chem. 94, 3820 (1990).

M. A. Thompson, M. C. Zerner, and J. Fajer, J. Phys. Chem. 95, 5693 (1991).

78 S. G. Boxer, Ann. Rev. Biophys. Chem. 19, 267 (1990).

79 J. Breton, Biochimica et Biophysica Acta 810, 235 (1985).

80 D. J. Lockhart and S. G. Boxer, Proc. Natl. Acad. Sci. 85, 107 (1988).

81 T. R. Middendorf, L. T. Mazzola, K. Lao, M. A. Steffen and S. G. Boxer, Biochimica et Biophysica Acta 1143, 223 (1993).

82 A. Vermeglio and G. Paillotin, Biochimica et Biophysica Acta 681, 32 (1982).

83 N. Raja, R. Stephen, S. V. Kolaczkowski, and G. J. Small, Science 260, 68 (1993).

84 S. W. DeLeeuw, J. W. Perram, and E. R. Smith, Proc. Roy. Soc. A 373, 27 (1980).

M. P. Allen and D. J. Tildesley, Computer Simulation of Liquids (Oxford University Press, New York, 1987).

86 G. Bonas, C. Sosso, and M. R. Vignon, Rapid Commun. Mass. Spectrom. 2, 88 (1988).

87 H. J. C. Berendsen, J. R. Grigera, and T. P. Straatsma, J. Phys. Chem. 91, 6269 (1987).

88 K. Watanabe and M. L. Klein, Chem. Phys. 131, 157 (1989).

89 L. X. Dang, J. Chem. Phys. 97, 2659 (1992).

90 F. MacRitchie, Chemistry at Interfaces (Academic Press, Inc., San Diego, California, 1990).

91 J. G. Kirkwood and F. P. Buff, J. Chem. Phys. 17, 338 (1949).

92 E. Salomons and M. Mareschal, J. Phys.: Condens. Matter 3, 3645 (1991).

93 A. H. Narten, J. Chem. Phys. 65, 573 (1976).
C. Pisani, R. Dovesi, and C. Roetti, Hartree-Fock Ab Initio Treatment of Crystalline Systems (Springer-Verlag, New York, 1988).

97 R. Dovesi, V. R. Saunders, and C. Roetti, Crystal92 User's Manual (Università di Torino, Torino, Italy, 1992).

98 M. Causà, R. Dovesi, C. Pisani, R. Colle, and A. Fortunelli, Phys. Rev. B 36, 891 (1987).

99 M. I. McCarthy and N. M. Harrison, Phys. Rev. B 49, 8574 (1994).

100 M. D. Towler, N. M. Harrison, and M. I. McCarthy, Phys. Rev. B 52, 5375 (1995).

101 L.-Q. Wang, D. R. Baer, and M. H. Engelhard, Surf. Sci. (1994).

102 M. A. Henderson, Surf. Sci 319, 315 (1994).

103 M. B. Hugenschmidt, L. Gamble, and C. T. Campbell, Surf. Sci. 302, 329 (1994)

104 C. A. Muryn, G. Tivengadum, J. J. Crouch, D. R. Warburton, G. N. Raiker, G. Thornton, and D. S.-L. Law, J. Phys.: Cond. Mat. 1, SB127 (1989).

105 C. A. Muryn, P. J. Hardman, J. J. Crouch, G. N. Raiker, G. Thornton, and D. S.-L. Law, Surf. Sci. 251/252, 747 (1991).

106 Laser Ablation: Mechanisms and Applications. II, Vol. 288, edited by J. C. Miller and J. R. F. Haglund (AIP Press, New York, 1994).

107 A Survey of Available Information on Gas Generation in Tank 241-101-SY, PNNL Report PN7520/AD-940, (Pacific Northwest National Laboratory, Richland, Washington, 1991).

108 R. A. Bradley, Jr., E. Lanzendorf, M. I. McCarthy, T. M. Orlando, and W. P. Hess, J. Phys. Chem. 99, 11715 (1995).

109 R. D. Poshusta, D. C. Tseng, A. C. Hess, and M. I. McCarthy, J. Phys. Chem. 97, 7295 (1993).

110 S. W. Peterson and H. A. Levy, Acta. Cryst. 10, 70 (1957).

111 Physics and Chemistry of Ice, Vol. , edited by E. Whalley, S. J. Jones, and L. W. Gold (Royal Society of Canada, Ottawa, 1973).

112 Y. Tajima, T. Matsuo, and H. Suga, Nature 299, 810 (1982).

113 A. J. Leadbetter, R. C. Ward, J. W. Clark, P. A. Tucker, and T. Maturo, J. Chem. Phys. 82, 424 (1985).

114 B. J. Yoon, K. Morokuma, and E. R. Davidson, J. Chem. Phys. 83, 1223 (1985).

115 D. A. Dixon and F. A. van Catledge, Int. J. Supercomputer Appl. 2, 62 (1988).

116 D. A. Dixon, J. Phys. Chem. 96, 3698 (1992).

117 W. H. Tuminello, T. A. Treat, and A. D. English, Macromol. 21, 2606 (1988).

118 Fluoropolymers, Vol., edited by L. A. Wall (John Wiley, New York, 1971).

119 M. F. Guest, E. Apra, D. E. Bernholdt, H. A. Fruechtl, R. J. Harrison, R. A. Kendall, R. A. Kutteh, X. Long, J. B. Nicholas, J. A. Nichols, H. L. Taylor, A. T. Wong, G. I. Fann, R. J. Littlefield, and J. Nieplocha, in High Performance Computing: Issues, Methods and Applications, 
edited by L. G. J. Dongarra, G. Joubert, and J. Kowalik (Elsevier Science, Amsterdam, 1995).

120 P. J. Flory, Statistical Mechanics of Chain Molecules (John Wiley, New York, 1969).

121 D. Chandler, in The Liquid State of Matter: Fluids, Simple and Complex, edited by E. W. Montroll and J. L. Lebowitz (North Holland, Amsterdam, 1982).

122 A. P. Smith, H. Yan, J. K. Wiggs, H. Jonsson, L. R. Corrales, P. Nachtigall, and K. D. Jordan, J. Chem. Phys. 102, 1044 (1995). 
Section 2

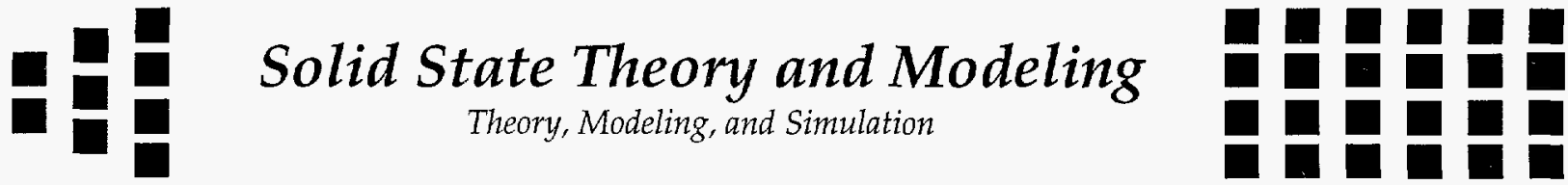

Many environmentally and industrially important systems are composed of one or more solid phases. To understand the behavior of these systems, it is necessary to understand the properties of the solid materials present and the chemistry and physics occurring at the solid/gas, solid/liquid, and solid/solid interfaces. The research focus in the Solid State Theory group is upon the development of accurate, predictive theoretical methods and their application to complex heterogeneous condensed phase systems. Application work in the program is directed toward the fields of geochemistry, geophysics, materials science, separation science, and heterogeneous catalysis. This embraces a diverse set of studies ranging from the determination of subsurface geochemical reactions and their molecular-level mechanisms to fundamental, atomic level investigations of catalytic processes at work in industrial, subsurface, and atmospheric environments.

\section{Development of Methodology for Modeling Solid State Systems}

There is a strong component of research in the Solid State Theory and Modeling program focused on understanding the properties of materials using techniques from $a b$ initio solid-state quantum chemistry and physics. Much of this work is motivated by the need for accurate and predictive theoretical techniques that can function reliably in the absence of empirical data. The scope of the effort includes the development and implementation of periodic Gaussian basis density functional theory to study chemical processes on the internal and external surfaces of catalytically and geochemically important materials. In the initial phases of this project a prototype nonperiodic Gaussian basis code was developed. That code was subsequently substantially modified and reworked to operate efficiently on massively parallel computer architectures by our development partners in the High Performance
Computational Chemistry Group (for a report of the status of the molecular code see Section 4 of this report). During the past two years we have concentrated on the final mathematical details of the calculation of periodic energies and first derivatives of the total energy with respect to nuclear coordinates for three, two, and one periodic dimension(s). In addition, we have generated the prototype three-dimensionally periodic total energy code. In what follows, a more detailed report is presented along with a statement of our near-term goals on this project.

Development Status of the Periodic Gaussian Basis Density Functional Methods (A. C. Hess, D. G. Clerc, ${ }^{(a)}$ Z. Lin, ${ }^{(b)}$ J. E. Jaffe, ${ }^{(c)}$ H. L. Taylor $^{(d)}$, and J. A. Nichols $\left.{ }^{(e)}\right)$

(a) AWU Graduate Research Associate

(b) AWU Postdoctoral Research Associate

(c) AWU Visiting Scientist

(d) AWU Postdoctoral Research Associate, High Performance Computational Chemistry Group

(e) High Performance Computational Chemistry Group

The past two years have seen considerable progress in the development of the Gaussian Basis Density functional modules, NWdft which is a portion of the NWChem code. This section of the report discusses some of the milestones met that are specific to the solid state capabilities of this program. Our overall design goals for this program include the ability to treat systems characterized by $0-, 1-, 2-$, or 3-dimensional periodicity. Our strategy in developing this new

The work reported in this section was supported by the Division of Geosciences, Office of Basic Energy Sciences and the Advanced Industrial Concepts, Division of Energy and Efficiency in the $U$. S. Department of Energy, and by Laboratory Directed Research and Development funds. Computational grants were provided at the National Energy Research Supercomputing Center by the Office of Scientific Computing in the U. S. Department of Energy. 
package has always been to reduce the formal order of the method during the mathematical formulation stage as much as possible and to implement the resulting theory on massively parallel computer architectures. In this way the next generation technology needed to treat many of the complex environmental and industrial systems of interest can be obtained. The final versions of this program will provide fully selfconsistent solutions to the Kohn-Sham equations and first derivatives of the resulting total energy with respect to nuclear coordinates. A host of properties (observables) which can be derived from these quantities are either planned or implemented.

Our principal goals for the solid-state modules during the past two years were mathematical and software development. We have now completed the mathematical formulation for the total energy to treat $0-3$ periodic dimensions. Periodic schemes for fitting exchange-correlation potentials and densities with auxiliary basis functions were refined, and a complete two-dimensional version of the periodic formalism was worked out. The equations for the two-dimensionally periodic case have the same form as those for three dimensions provided that lattice and reciprocal vectors are interpreted as two-dimensional vectors (in the plane of a surface or slab) while atomic position vectors within a unit cell and real-space integrals remain three dimensional. Also, a different form of the Ewald potential must be used. ${ }^{1}$ In addition, we have completed the formulation of the atomic forces (first derivatives of the energy with respect to nuclear coordinates) for all periodic dimensions. The software development activities during the past two years have both focused on implementing these equations in first generation code as well as developing and refining software to fully exploit any space group symmetry the object may have. This includes Brillouin zone integration, screening, and selection algorithms and a numerical integration schemes suited to periodic systems. As the testing phase of the sequential three-dimensional code comes to completion, effort will be devoted to the development of fully distributed massively parallel processor (MPP) versions of the algorithms. Our principal goals for the upcoming year include the implementation of algorithms capable of calculating atomic forces for three-dimensionally periodic objects and the implementation of the analogous quantities (energy and first derivatives) for two dimensionally periodic systems (surfaces).

The standard density functional theory for analytical gradients (forces) in molecular systems was also extended formally to periodic systems. Denoting a general nuclear coordinate as $y$, the derivative of the total energy per unit cell can be written as

$$
\begin{aligned}
\frac{d E}{d y} & =\sum_{i j R} P_{i j}(R)\left\{\frac{d H_{i j}^{k i n}(R)}{d y}+\frac{d C_{i j p}(R)}{d y}\right. \\
& \left.+\int V_{X C}[\rho] \frac{d}{d y}\left[\chi_{i}(r) \chi_{j}(r-R)\right] d^{3} r\right\} \\
& +\sum_{p} a_{p} \frac{d T_{p}}{d y}-\frac{1}{2} \sum_{p p^{\prime}} a_{p} \frac{d V_{p p^{\prime}}}{d y} a_{p^{\prime}} \\
& -\sum_{i j} P_{i j}^{\varepsilon}(R) \frac{d S_{i j}(R)}{d y}
\end{aligned}
$$

where $\mathrm{V}_{\mathrm{XC}}$ is the exchange-correlation potential, $\mathrm{T}_{\mathrm{p}}$ and $\mathrm{V}_{\mathrm{pp}}$ involve one and two center Coulomb interactions of density fitting functions, $C_{i j p}$ involves repulsion integrals between orbital and fitted density functions, $a_{p}$ is a density fitting coefficient, $\mathbf{R}$ is a direct lattice vector and the $\chi$ denotes an orbital basis function. Also $P_{i j}$ is the density matrix, $S_{\mathrm{ij}}$ is the orbital basis overlap, and the energy-weighted density matrix is given by

$$
P_{\mathrm{ij}}^{\varepsilon}(R)=\sum_{\mathrm{k}} w_{k} \sum_{\mathrm{n}}^{\mathrm{occ}} \varepsilon_{\mathrm{kn}} e^{-\mathrm{ik} \cdot R} \mathrm{c}_{\mathrm{kni}}^{*} c_{\mathrm{knj}}
$$

where the sum is over a mesh of reciprocal space points in the first Brillouin zone, $w_{k}$ is a weight factor, $\varepsilon_{\mathrm{kn}}$ is the band energy, and the c's are the orbital coefficients. The software implementation of these equations is well under way.

A Multicenter Integration Scheme for Electronic Structure Calculations of Periodic and NonPeriodic Polyatomic Systems (Z. Lin ${ }^{(a)}$ and A. C. Hess)

(a) AWU Postdoctoral Research Associate

Numerical integration is a necessity in calculations using density functional theory (DFT). The most widely implemented numerical integration 
method requires a CPU time proportional to the cube of the number of atoms in the molecule, accompanied with a large prefactor, and must be modified before applied to extended systems. An efficient and flexible numerical integration scheme would be very beneficial for computations on large molecules and periodic systems. Our effort in developing a solid-state DFT code, which is aimed at applications on large systems, motivated this work.

The most CPU-intensive part in numerical integration is the determination of matrix element, $\mathrm{A}=\int \psi_{\mathrm{i}} \mathrm{V} \psi_{\mathrm{j}} \mathrm{d} \overrightarrow{\mathrm{x}}$. Here $\psi_{\mathrm{i}}$ and $\psi_{\mathrm{j}}$ are atomic orbitals centered at nuclei $i$ and $j$, respectively. $V$ is the exchange-correlation potential or the KohnSham effective potential. Characterized with density cusps and/or Coulomb singularities at nuclei, the potential, $V$, exhibits centralized features at regions near all atomic centers and these regions dominate the value of integral, A. However, for atoms that are far away from $\mathrm{i}$ and/or $j$, the multi-center feature of $V$ is suppressed by the smallness of $\psi_{\mathrm{i}} \psi_{\mathrm{j}}$, and its contribution to $\mathrm{A}$ is small. To take advantage of this feature, we first partition the potential into a sum over atomic contributions. The sum is then grouped with contributions from atoms, denoted as $\mathrm{Ks}$, neighboring to both nuclei $i$ and $j$ and the rest. The integral is thus transformed into a practical two-center integral and a sum over some three-center integrals. Two-center partition is used to decompose each of these three-center integrals to two two-center integrals. Each twocenter integral is further decomposed into two one-center integrals. After grouping all these resulting one-center integrals, with some algebra, we have,

$$
\begin{aligned}
A= & \int \psi_{\mathrm{i}} \mathrm{V} \psi_{\mathrm{j}} \mathrm{g}_{\mathrm{ij}}^{\mathrm{I}} \mathrm{d} \overrightarrow{\mathrm{x}}_{\mathrm{I}}+\int \psi_{\mathrm{i}} \mathrm{V} \psi_{\mathrm{j}} \mathrm{g}_{\mathrm{ij}}^{\mathrm{J}} \mathrm{d} \overrightarrow{\mathrm{x}}_{\mathrm{J}} \\
& +\sum_{\mathrm{K}} \int \psi_{\mathrm{i}} \mathrm{V} \psi_{\mathrm{j}} \mathrm{g}_{\mathrm{ij}}^{\mathrm{K}} \mathrm{d} \vec{x}_{\mathrm{K}}
\end{aligned}
$$

where,

and

$$
\mathrm{g}_{\mathrm{ij}}^{\mathrm{I}}=\mathrm{S}_{\mathrm{IJ}}\left\{1+\sum_{\mathrm{K}}\left(\mathrm{S}_{\mathrm{IJ}}-1\right) \omega_{\mathrm{K}}\right\}
$$

$$
\mathrm{g}_{\mathrm{ij}}^{\mathrm{K}}=\omega_{\mathrm{K}}\left\{\mathrm{S}_{\mathrm{IJ}} \mathrm{S}_{\mathrm{KI}}+\mathrm{S}_{\mathrm{JI}} \mathrm{S}_{\mathrm{KJ}}\right\} .
$$

In other words, the multi-center integral $\int \psi_{\mathrm{i}} V \psi_{\mathrm{j}} \mathrm{d} \overrightarrow{\mathrm{x}}$ is decomposed into a sum of onecenter integrals at site $i$ and $j$ and Ks which are within certain range of both $i$ and $j$. The cost for each integral will then not increase for systems exceeding some certain size. In fact, for $\int \psi_{\mathrm{i}} V \psi_{\mathrm{j}} \mathrm{d} \overline{\mathrm{x}}$ with increasing $\mathrm{i}-\mathrm{j}$ distance, the number of common neighbors decreases and the average cost per integral decreases with increasing system sizes compared to systems of medium size. For very large systems, the number of nonnegligible matrix elements increases linearly with the number of atoms in the systems. In such cases, the numerical integration costs in our scheme increase linearly and scale very well for large systems. Moreover, our scheme can readily be applied to periodic systems.

In addition to the improvement of the general integration scheme, one-center meshes were critically examined and efficient grids were designed. This included refinement of radial meshes, combination of radial-angular meshes and automatic mesh generators. Combined with the above method, substantial reductions in number of mesh points were achieved. Table 2.1 gives some comparison of our scheme and that of Perez-Jorda, Becke, and San-Fabian, ${ }^{2}$ the one currently in widest use. The integrands tested are total charge densities. This is in fact an unfavorable quantity for our method with large systems as no reduction in number of atoms centers, like that in matrix element evaluations, is possible here. (The numbers of mesh points needed for matrix element determinations and other quantities in the scheme of Perez-Jorda et al. are identical.). Nevertheless, Table 2.1 shows clearly that our method is advantageous. The significant improvement arises mainly from two factors: improved one-center meshes as mentioned above, and, equally important, the improved angular behavior of the resulting one-center integrands. The latter is not obvious in the formalism but is evident by plotting out the 1center integrands. Table 2.2 shows the maximum numbers of grid points needed with our method in matrix element evaluations for $\mathrm{C}_{60}, \mathrm{C}_{240}$, and the $\mathrm{MgO}$ crystal. The boundary between "near" and "distant" neighbors in our scheme is drawn at a radius of 10 a.u. For $\mathrm{C}_{60}$, a reduction in mesh points of about a factor of 20 is achieved, compared to the method of Perez-Jorda et al. (see Table 2.1). Even bigger savings are realized for $\mathrm{C}_{240}$ as at least a factor of four times as many mesh points as that of $C_{60}$ are needed in the scheme of Perez-Jorda et al. In fact, no apparent increase in mesh points for matrix element evaluations can be seen on going from medium 
size systems (Table 2.1) to large ones as the latter benefited from our scheme much more than the former. Moreover, our method applies to periodic systems naturally whereas modification must be made to the scheme of Perez-Jorda et al. before application to periodic systems.

The cutoff radius for "near" neighbors is adjustable. Reducing the cutoff radius in general would enlarge the number of one-center meshes needed but reduce the number of atom centers in integrations. The tradeoff may provide an overall reduction of total cost for accuracies needed in practice, say, up to an absolute accuracy of $10^{-5}$ a.u. For comparison, the numbers in parentheses in Table 2.2 are the numbers of grid points needed corresponding to a cutoff radius of 7 a.u.

In summary, we have found a multi-center integration scheme which greatly reduces the CPU requirement for large systems.

Table 2.1. Comparison of integration efficiency. Shown are the total numbers of grid points needed for integration of densities at five absolute tolerances (no symmetry is used).

\begin{tabular}{|c|c|c|c|c|c|}
\hline & \multicolumn{5}{|c|}{ tolerance } \\
\hline & $10^{-3}$ & $10^{-4}$ & $10^{-5}$ & $10^{-6}$ & $10^{-7}$ \\
\hline \multicolumn{6}{|l|}{$\mathrm{H}_{2} \mathrm{O}$} \\
\hline Perez-Jorda et al. ${ }^{2}$ & 5202 & 15714 & 30726 & 81042 & 151206 \\
\hline new scheme & 1065 & 2695 & 4948 & 7825 & 11436 \\
\hline \multicolumn{6}{|l|}{$\mathrm{C}_{6} \mathrm{H}_{6}:$} \\
\hline Perez-Jorda et al. ${ }^{2}$ & 37920 & 63336 & 219000 & 746760 & 1127064 \\
\hline new scheme & 5936 & 12121 & 22349 & 37738 & 72509 \\
\hline \multicolumn{6}{|l|}{$\mathrm{ZnCl}_{3} \mathrm{C}_{5} \mathrm{H}_{5} \mathrm{~N}_{5}:$} \\
\hline Perez-Jorda et al. ${ }^{2}$ & 43022 & 177614 & 651188 & 1206932 & 2516828 \\
\hline new scheme & 6225 & 11834 & 22699 & 67840 & 109070 \\
\hline \multicolumn{6}{|l|}{$\mathrm{C}_{15} \mathrm{H}_{12} \mathrm{~N}_{2} \mathrm{O}_{2}:$} \\
\hline Perez-Jorda et al. ${ }^{2}$ & 96058 & 293812 & 1058548 & 2138980 & 5383486 \\
\hline $\begin{array}{l}\text { new scheme } \\
\mathrm{C}_{17} \mathrm{H}_{21} \mathrm{NO}_{4}:\end{array}$ & 12584 & 26387 & 44360 & 79836 & 180675 \\
\hline Perez-Jorda et al. ${ }^{2}$ & 150406 & 546598 & 1514914 & 3865324 & 7799812 \\
\hline new scheme & 9573 & 21008 & 38657 & 77423 & 165136 \\
\hline \multicolumn{6}{|l|}{$\mathrm{C}_{60}:$} \\
\hline Perez-Jorda et al. ${ }^{2}$ & 243240 & 5802900 & 802320 & 5814600 & 16872000 \\
\hline new scheme & 28098 & 79296 & 133898 & 199916 & 332337 \\
\hline
\end{tabular}

Table 2.2. Largest number of grid points needed for a matrix element evaluation. Cutoff radius used is 10 a.u. Numbers inside parentheses are those for cutoff radius of 7 a.u. No symmetry is used.

\begin{tabular}{cccccc} 
& \multicolumn{5}{c}{ tolerance } \\
\cline { 2 - 6 } & $10^{-3}$ & $10^{-4}$ & $10^{-5}$ & $10^{-6}$ & $10^{-7}$ \\
\hline $\mathrm{C}_{60}$ & 12079 & 26582 & 57904 & 148052 & 505333 \\
& $(6997)$ & $(15616)$ & $(53007)$ & $(162458)$ & \\
$\mathrm{C}_{240}$ & 11386 & 38291 & 57793 & 165434 & 495464 \\
& $(4321)$ & $(14343)$ & $(81522)$ & $(226418)$ & \\
$\mathrm{MgO}$ & 21861 & 53931 & 143366 & 346593 & 906086 \\
(crystal) & $(7455)$ & $(18898)$ & $(53185)$ & $(309124)$ & $(557334)$
\end{tabular}




\section{Chemistry and Physics of Materials}

CO Adsorption on the Nonpolar (1010) Surface of ZnO (J. E. Jaffe ${ }^{(a)}$ and A. C. Hess)

(a) AWU Visiting Scientist

Periodic Hartree-Fock (PHF) total energy calculations on two-dimensionally periodic slabs have been used to predict the equilibrium geometry of a monolayer of $\mathrm{CO}$ molecules adsorbed on the nonpolar (10 $\overline{10})$ surface of $\mathrm{ZnO}$. Large contracted Gaussian all-electron basis sets were used for both the slab and the molecules, and the initial geometry was taken to consist of a $C O$ vertically oriented over one of the surface atoms in each $\mathrm{ZnO}$ dimer, with the positions of the latter relaxed as in the corresponding clean surface. ${ }^{3}$ The resulting geometry of both the $\mathrm{ZnO}$ surface and the $\mathrm{CO}$ molecule were then fully optimized. During the course of the unconstrained optimization processes, the $\mathrm{ZnO}$ surface was not observed to undergo significant further relaxation in the presence of the $\mathrm{CO}$ molecules. Of principal interest is the angle that the $\mathrm{CO}$ molecule makes with respect to a direction perpendicular to the surface. Good agreement is obtained between the experimental $\left(\phi=30^{\circ}\right)$ and theoretical $\left(\phi=39^{\circ}\right)$ values. The value of the angle $\theta$ is of little comparative use as it was assumed (not determined) by the experimental study to be $30^{\circ}$ (thus our value of $\theta=32^{\circ}$ constitutes a new prediction). In addition to predicting the angle $\theta$, we also predict that the carbon to zinc distance is approximately $2.7 \AA$. The two sets of vibrational frequencies reported constitute $\mathrm{CO}$ motion relative to the $\mathrm{ZnO}$ surface, $v_{1}$ and internal $\mathrm{CO}$ motion, $v_{2}$. Although there is reasonable agreement in absolute magnitude, the relative change in the internal $\mathrm{CO}$ vibrational frequency from its gas phase value is of particular interest. Experimental results indicate an increase in this frequency by approximately $5.8 \% .{ }^{4}$ Our theoretical results predict an increase in the frequency of motion by approximately $3.5 \%$. Not surprisingly experiment shows that the bond length upon adsorption of the $\mathrm{CO}$ molecule decreases by 0.03 $\AA$ and the theoretical shortening is $0.01 \AA$. The bond length contraction and subsequent frequency increase are interpreted to result from a very small charge transfer from anti-bonding molecular orbitals in the $\mathrm{CO}$ to the $\mathrm{Zn}$ atoms. This strengthening of the intramolecular bond is unusual for surface-bound species and has important implications for the catalytic properties of the surface. For example, upon hydrogenation one obtains methanol on $\mathrm{ZnO}$ versus methane and water as found on most metal surfaces since the strengthened bond on $\mathrm{ZnO}$ is not cleaved.

From several experimental measurements and a rather involved set of arguments Solomon et al. 4 concluded that the $\mathrm{CO}$ molecule prefers to bind "carbon end down" to the $\mathrm{Zn}$ atom on the surface. In order to evaluate this prediction we also performed detailed studies in which the $\mathrm{CO}$ molecule approached the surface with the "oxygen end down." The results of our study ${ }^{3}$ indicate that both configurations show a net binding to the surface. However, the minimum energy configuration corresponding with the carbon atom closest to the surface results in a 10 $\mathrm{kcal} / \mathrm{mol}$ adsorption energy in the low concentration limit whereas an oxygen down configuration results in an adsorption energy of 9 $\mathrm{kcal} / \mathrm{mol}$. The experimental heat of adsorption is reported to be $12 \mathrm{kcal} / \mathrm{mol}$ and we therefore agree that there is a slight preference for the $\mathrm{CO}$ molecule to adsorb carbon end down to the surface. In summary, there is very good agreement between experiment and theory on the four observable quantities just discussed.

Periodic Hartree-Fock Study of $\mathrm{Li}_{x} \mathrm{TiS}_{2}, 0 \leq x \leq 1$ (D. G. Clerc, ${ }^{(a)}$ R. D. Poshusta, ${ }^{(b)}$ and A. C. Hess)

(a) AWU Graduate Research Associate

(b) AWU Faculty Research Associate

$\mathrm{Li}_{\mathrm{x}} \mathrm{TiS}_{2}$ belongs to the family of alkali-intercalated transition metal dichalcogenides $\mathrm{A}_{\mathrm{x}} \mathrm{MCh}_{2}$ $(\mathrm{A}=$ alkali metal; $\mathrm{M}=$ transition metal; $\mathrm{Ch}=\mathrm{S}$, $\mathrm{Se}$, or $\mathrm{Te}$ ). Several of these compounds have been extensively studied because of their potential use as cathode materials in ambient-temperature highenergy density batteries..$^{5-7}$ Of these compounds, $\mathrm{Li}_{\mathrm{x}} \mathrm{TiS}_{2}$ is the most promising because of its nearly ideal electrochemical properties. ${ }^{8}$

The unusual affinity of $\mathrm{MCh}_{2}$ for intercalation is due to their characteristic bonding anisotropy. For example, in $\mathrm{TiS}_{2}$ the $\mathrm{Ti}$ atoms lie in planes, each of which is sandwiched between two parallel planes of hexagonally packed $\mathrm{S}$ atoms. The bonding within these S-Ti-S lamellae or layers is strongly covalent. In contrast, the coupling of 
strongly covalent. In contrast, the coupling of adjacent layers is relatively weak. Consequently, the intercalated species is inserted into the interlayer space - commonly called the van der Waals gap - where the bonding is weaker.

The structural and electronic properties of $\mathrm{Li}_{\mathrm{x}} \mathrm{TiS}_{2}, \mathrm{x}=0,0.25,0.75$ and 1.00 , were investigated using CRYSTAL92. 9,10 All-electron PHF (self-consistent-field linear combination of atomic orbitals) calculations were performed and electron correlation effects were added by $a$ posteriori density functional correlation corrections to the PHF total energies. These studies include both the semiconductor-to-semi-metal phase transition in neat-TiS 2 and the effects of lithium intercalation on the lattice parameters, bulk moduli, linear moduli, cohesive energy, elastic constants, band structure, density of states, and Mulliken populations.

The pressure induced semiconductor-to-semimetal phase transition in neat- $\mathrm{TiS}_{2}$ is found to result from an indirect band overlap between a sulfur $3 p_{z}$-based valence band and a titanium $3 d$ based conduction band. The pressure shift of this overlap in the metallic phase is predicted to be 30 $\mathrm{meV} / \mathrm{GPa}$, which is similar to the experimental value of $45 \pm 15 \mathrm{meV} / \mathrm{GPa}$. However, the phase transition is predicted to occur at a pressure of 84 $\mathrm{GPa}$ - far greater than the experimental value of $4.0 \pm 0.5 \mathrm{GPa}$. This sizable error is due to the large overestimation of the zero-pressure optical band gap in the Hartree-Fock approximation. Correcting this energy gap by using empirical rigid-band shifts results in a predicted transition pressure between 1.5 and $4.0 \mathrm{GPa}$, which is consistent with experiment. Lattice parameters a, $c$, and $\mathrm{z}$ at the phase transition are predicted to be $5.42(3) \AA, 3.284(5) \AA$, and $0.2567(16)$, respectively.

The $\mathrm{Li}_{\mathrm{x}} \mathrm{TiS}_{2}$ calculations indicate the following effects of intercalation: 1) a large uniaxial expansion in the direction perpendicular to the layers, 2) a negligible (large) layer expansion at $x=0.25$ $(x=1), 3)$ negligible changes in the elastic properties at $x=0.25$ and significant strengthening of the interlayer bonding at $x=1,4)$ charge transfer from lithium to the $\mathrm{S}(3 \mathrm{p})$ and $\mathrm{Ti}(3 \mathrm{~d})$ orbitals - with at least $75 \%$ being distributed on the sulfur atoms, $5)$ a decrease in the $\operatorname{Ti}(3 \mathrm{~d})$ contribution to the valence bands, 6) charge transfer to a $\mathrm{Ti}(3 \mathrm{~d})$ based conduction band which results in metallic behavior, and 7$)$ the donation of charge to the $\mathrm{Ti}\left(3 \mathrm{~d}_{z^{2}}\right)$ orbital at small $x(x<0.25)$.

Periodic Hartree-Fock Pseudopotential Study of $\mathrm{Li}_{\mathrm{x}} \mathrm{Cd}_{2} \mathrm{P}_{2} \mathrm{~S}_{6}, \mathrm{x}=\mathbf{0}, 1,2$, and 3 (D. G. Clerc, ${ }^{(a)} \mathrm{R}$. D. Poshusta, ${ }^{(b)}$ and A. C. Hess)

(a) AWU Graduate Research Associate

(b) AWU Faculty Research Associate

Cadmium phosphorous sulfide, $\mathrm{Cd}_{2} \mathrm{P}_{2} \mathrm{~S}_{6}$ crystallizes in a layered structure. Each layer consists of $\mathrm{Cd}$ atoms and $\mathrm{P}_{2}$ dimers which occupy the octahedral sites between two close-packed planes of $S$ atoms. The layers are stacked such that the sulfur atoms form a cubic close-packed array. ${ }^{11}$ The weak bonding across the interlayer space allows the intercalation of various molecules and metal atoms into the crystal.

The effects of charge-transfer intercalation upon the structural and bulk electronic properties of neat- $\mathrm{Cd}_{2} \mathrm{P}_{2} \mathrm{~S}_{6}$ were investigated using pseudopotential PHF theory. These calculations were performed using CRYSTAL92, 9,10 - a welldocumented PHF self-consistent field linearcombination of atomic orbitals program. This program can operate using effective core potentials (ECP) as well as the all-electron approach. In this study, the ECP approach was used and $a$ posteriori density functional correlation corrections were applied to the PHF total energies to correct for electron correlation.

Lattice parameters, bond lengths, Mulliken populations, band structures, and densities of states were calculated for the intercalation compound $\mathrm{Li}_{\mathrm{x}} \mathrm{Cd}_{2} \mathrm{P}_{2} \mathrm{~S}_{6}, \mathrm{x}=0,1,2$, and 3. The $\mathrm{Cd}_{2} \mathrm{P}_{2} \mathrm{~S}_{6}$ acceptor level (the crystal orbital which contains the donated electron) at $\mathrm{x}=1$ was also calculated. The identity of the acceptor level is important in studies of the charge transfer mechanism of intercalation in $\mathrm{Cd}_{2} \mathrm{P}_{2} \mathrm{~S}_{6}$ and related compounds.

The principal effects of intercalation can be summarized as follows. 1) The intralayer lattice parameters increase with increasing $x$. 2) The perpendicular distance between layers increases at $x=1$, remains the same at $x=2$, and decreases at $x=3$. 3) The layers shear by $e_{13}=1 / 6$ at $x=2$. 4) Marked distortion of the $\mathrm{PS}_{3}$ tetrahedra occurs at $x=2,3.5)$ In each $P_{3}$ tetrahedra, the character of one P-S bond changes from strongly bonding at 
bonding at $\mathrm{x}=0,1,2$, and 3. 6) $\mathrm{Cd}$ is oxidized and $S$ is chemically reduced thus inducing increased layer ionicity. 7) The acceptor level is derived from $P(3 s)$ orbitals and the nearest-neighbor $S\left(3 p_{x}\right)$ and $S\left(3 p_{y}\right)$ orbitals oriented toward the $P(3 s)$ orbital.

\section{Chemistry and Physics of Catalysts}

Microporous aluminosilicate zeolites are important catalysts used in petroleum refining and fine chemical synthesis. The catalytic function of zeolite is largely attributed to the presence of Brønsted acid sites located in the zeolite channels and cages. However, the zeolite has other notable features that influence reactivity. For example, the presence of extra framework aluminum and metal cations in the zeolite can provide Lewis acidity. In addition, the steric constraints imposed by the zeolite channels can affect reactions by slowing the motion of reactants and products, giving high shape selectivity. The zeolite may also influence product mixtures by preventing the formation of bulky transition states. The well-defined geometries of the zeolite crystals provide an excellent starting point for high level calculations of zeolite structure and function. In addition, a variety of other experiment techniques can provide valuable information to test and verify theoretical work. Our recent work in zeolite catalysis is presented below.

Local Density Functional Studies of [T]-ZSM-5, $\mathrm{T}=\mathrm{Si}, \mathrm{B}, \mathrm{Al}$, Ga, and Fe. (M. S. Stave ${ }^{(a)}$ and J. B. Nicholas)

(a) Cray Research Inc., Eagan, Minnesota

We have done a series of studies involving the application of DFT to zeolite chemistry. A considerable body of literature indicates that DFT gives results similar to post Hartree-Fock methods (second-order Møller-Plesset perturbation theory, MP2) at considerably less computational cost; correlated $a b$ initio methods scale formally as $\mathrm{N}^{5}$ or worse while DFT scales as $N^{3}$. Efficient algorithms lower the effective cost of either method, but DFT always retains its considerable computational advantage. Considering their large size (units cells contain- ing 300 atoms or more) DFT could provide the method of choice for obtaining accurate information about zeolites. Our earlier work showed that results from DFT were in good agreement with MP2 calculations using similar basis sets. ${ }^{12,13}$ Here we explore the structure and acidity of a series of substituted ZSM-5 models.

The replacement of $\mathrm{Si}$ in the siliceous zeolite framework by various trivalent atoms gives zeolites their Bronsted acidic function. The use of different substituent atoms, such as $\mathrm{Al}, \mathrm{B}, \mathrm{Ga}$, and $\mathrm{Fe}$, varies the acidity of the framework, allowing the catalytic function of the zeolite to be tuned for different applications. We calculated the deprotonation energy $\left(\Delta E_{0}\right)$ for a series of substituted ZSM-5 models and compared it to the acidity trend experimentally determined by $\mathrm{Chu}$ and Chang. ${ }^{14}$ Calculations were done on $\left(\mathrm{OH}_{3} \mathrm{Si}\right.$ $\mathrm{O}_{3}-\mathrm{T}-\mathrm{O}(\mathrm{H})-\mathrm{Si}-\left(\mathrm{O}-\mathrm{Si}(\mathrm{OH})_{3}\right)_{3}$ models, $\mathrm{T}=\mathrm{Al}, \mathrm{Ga}$, and $\mathrm{Fe}$, using the program $\mathrm{Dmol}^{15}$ and a DNP+ basis set. ${ }^{16}$ The central Brønsted acid site and the next neighbor shell of oxygens were optimized while the remaining atoms were held fixed in the crystal positions. In Table 2.3 we show the calculated $\Delta \mathrm{E}_{0}$ and charge on the acidic proton compared to the experimental IR frequencies. As the table shows, both theoretical measures of zeolite acidity are in excellent agreement with the experimental trend, strongly supporting the proposal that acidity decreases in the order $\mathrm{Al}>$ $\mathrm{Ga}>\mathrm{Fe}>>\mathrm{B}$. Our DFT calculations of the siliceous zeolite give geometries that agree well with the x-ray crystal structure. This work is further proof of the usefulness of DFT methods in the study of zeolites.

Table 2.3. Theoretical and Experimental Measures of Zeolite Acidity.

\begin{tabular}{lcccc} 
& $\mathrm{Al}$ & $\mathrm{Ga}$ & $\mathrm{Fe}$ & $\mathrm{B}$ \\
\hline$\Delta \mathrm{E}_{0}(\mathrm{kcal} / \mathrm{mol})$ & 319.3 & 322.3 & 325.2 & 337.3 \\
$\mathrm{q}(\mathrm{H})(\mathrm{lel})$ & 0.254 & 0.242 & 0.237 & 0.194 \\
$v(\mathrm{O}-\mathrm{H})\left(\mathrm{cm}^{-1}\right)$ & 3610 & 3620 & 3630 & 3745
\end{tabular}


Comparison of Density Functional and $A b$ Initio Methods for the Determination of Transition State Geometries and Energetics ( $Q$. Zhao $^{(a)}$ and J. B. Nicholas)

(a) AWU Postdoctoral Research Associate

Density functional methods use a mathematical representation of electron exchange and correlation. In the simplest case, so-called local density functional methods use exchange-correlation functionals based on the electron density. Better results are usually obtained with functionals that include the electron density gradient (nonlocal or gradient-corrected functionals). However, recent work has shown that even the nonlocal functionals can be improved upon by including the exact Hartree-Fock exchange in some parameterized fashion. In this work we study the geometries and energetics of the gas phase addition of $\mathrm{HF}$ to trans-2-butene using a variety of exchange-correlation functionals and compare to post Hartree-Fock results. We are particularly interested in the geometry of the transition state and the reaction barrier. The states along the reaction, and representative values of the energy differences, are shown in Figure 2.1.

In Table 2.4 we show the energies of the van der Waals complex, transition state, and reaction product relative to the energies of the reactants at

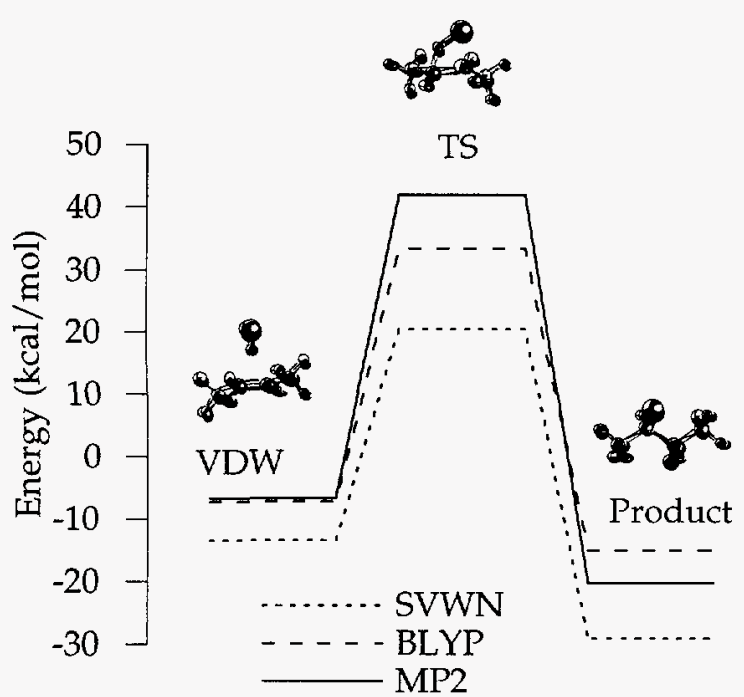

Figure 2.1. The reactants, van der Waals complex, transition state, and products for the addition of HF to trans-2-butene. infinite separation. All transition states were verified by frequency calculations. As seen in the table, the energies are very sensitive to the representation of exchange and correlation. For example, the local functionals (SVWN, HL-JMW) give much stronger interaction energies for the van der Waals state than any of the other calculations, and predict reaction barriers of approximately 34 $\mathrm{kcal} / \mathrm{mol}$. The inclusion of nonlocal corrections (BLYP) gives a much less tightly bound van der Waals state and increases the reaction barrier to $\approx 40 \mathrm{kcal} / \mathrm{mol}$. The result closest to those obtained at MP2 and MP4 is given by the B3LYP functional, which includes some percentage of the exact exchange. Similar results are obtained for the geometries of the various states along the reaction. Although a more accurate post HartreeFock result would provide a better benchmark,

Table 2.4. Theoretical energies $(\mathrm{kcal} / \mathrm{mol})$ of the states along the reaction path relative to the isolated reactants.

\begin{tabular}{|c|c|c|c|c|}
\hline Method & VDW & TS & Product & $\begin{array}{l}\text { Energy } \\
\text { barrier }\end{array}$ \\
\hline $\begin{array}{l}\text { SVWN }^{a} \\
\text { /DNP }^{b}\end{array}$ & -13.5 & 20.5 & -29.2 & 34.0 \\
\hline $\begin{array}{l}\text { HL-JMW } \\
/ \mathrm{DNP}^{b}\end{array}$ & -13.3 & 20.4 & -29.1 & 33.7 \\
\hline $\begin{array}{l}\text { BLYP } \\
/ \mathrm{DNP}^{b}\end{array}$ & -7.2 & 33.4 & -15.1 & 40.6 \\
\hline $\begin{array}{l}\text { BLYPc } \\
/ 6-31+G^{*}\end{array}$ & -5.8 & 33.2 & -17.4 & 39.0 \\
\hline $\begin{array}{l}\text { Beck3LYPd } \\
/ 6-31+\mathrm{G}^{*}\end{array}$ & -6.1 & 36.8 & -20.4 & 42.9 \\
\hline $\begin{array}{l}\mathrm{MP2} 2^{e} \\
/ 6-31+\mathrm{G}^{*}\end{array}$ & -6.7 & 42.0 & -20.3 & 48.7 \\
\hline $\begin{array}{l}\mathrm{MP} 4^{f} \\
/ 6-31+\mathrm{G}^{*}\end{array}$ & -6.6 & 39.3 & -21.4 & 45.9 \\
\hline
\end{tabular}

a Slater exchange and Vosko-Wilk-Nossair correlation functional. ${ }^{17}$

$b \quad$ Double zeta polarized basis set. ${ }^{16}$

c Becke exchange and Lee-Yang-Parr correlation. ${ }^{18,19}$

d Hybrid functional. ${ }^{20}$

$e \quad$ Second-order Møller-Plessett perturbation theory. 21

$f \quad$ Fourth-order Møller-Plessett perturbation theory. ${ }^{21}$ 
our results indicate that the hybrid functionals give an increase in accuracy over conventional local and nonlocal functionals.

Unified NMR and DFT Study of Hammett Bases in Acidic Zeolites (J. F. Haw, ${ }^{(a)}$ J. B. Nicholas, L. W. Beck, ${ }^{(a)}$ T. R. Krawietz, ${ }^{(a)}$ and D. B. Ferguson ${ }^{(a)}$ )

(a) Laboratory for Magnetic Resonance and Molecular Science, Department of Chemistry, Texas A\&M University

Until recently, the mechanism of action of acidic zeolites was not disputed. Because they catalyze the cracking of hexane and other profoundly weak bases at elevated temperatures, zeolites are widely regarded as Brønsted superacids. In this work we report nuclear magnetic resource (NMR) and DFT studies of Hammett indicator bases in acidic zeolites. These results provide clear proof that the acid strength of zeolites is well below that of superacids.

The acid strength of zeolites is determined by the protonation state of adsorbed Hammett indicators. Superacids are defined as those that can protonate Hammett bases with $\mathrm{H}_{\mathrm{BH}^{+}}$values of -12 (the same as $100 \% \mathrm{H}_{2} \mathrm{SO}_{4}$ ) or lower. Figure 2.2 shows the optimized geometry for $p$-fluoronitrobenzene (PFNB) in contact with a zeolite acid site. The $\mathrm{H}_{\mathrm{BH}}{ }^{+}$value of PFNB is -12.4 , just over the threshold of superacidity. As the figure shows, PFNB is not protonated by the zeolite, in agreement with the results of ${ }^{19} \mathrm{~F}$ NMR experiments. Both DFT and NMR also indicate that the slightly stronger base, p-nitrotoulene $\left(\mathrm{H}_{\mathrm{BH}^{+}}=\right.$ -11.4) is also not protonated in highly acidic zeolites. NMR chemical shift calculations on the PFNB complex give a ${ }^{19} \mathrm{~F}$ shift that is only 2.6 ppm from that of the neutral PFNB molecule, consistent with the lack of protonation and establishing quantitative agreement between experiment and theory. Although we cannot rule out the effects on reactivity provided by Lewis sites, the results are unequivocal proof that zeolites do not possess superacidity in the simple Brønsted sense. Preliminary DFT calculations of the PFNB complex interacting with a zeolite model with fixed Si-OH-Al angles cast doubt on the possibility of any aluminosilicate zeolites with simple Brønsted sites of superacid strength; even for an $\mathrm{Si}-\mathrm{OH}-\mathrm{Al}$ angle of $180^{\circ}$ the zeolite does not protonate PFNB.

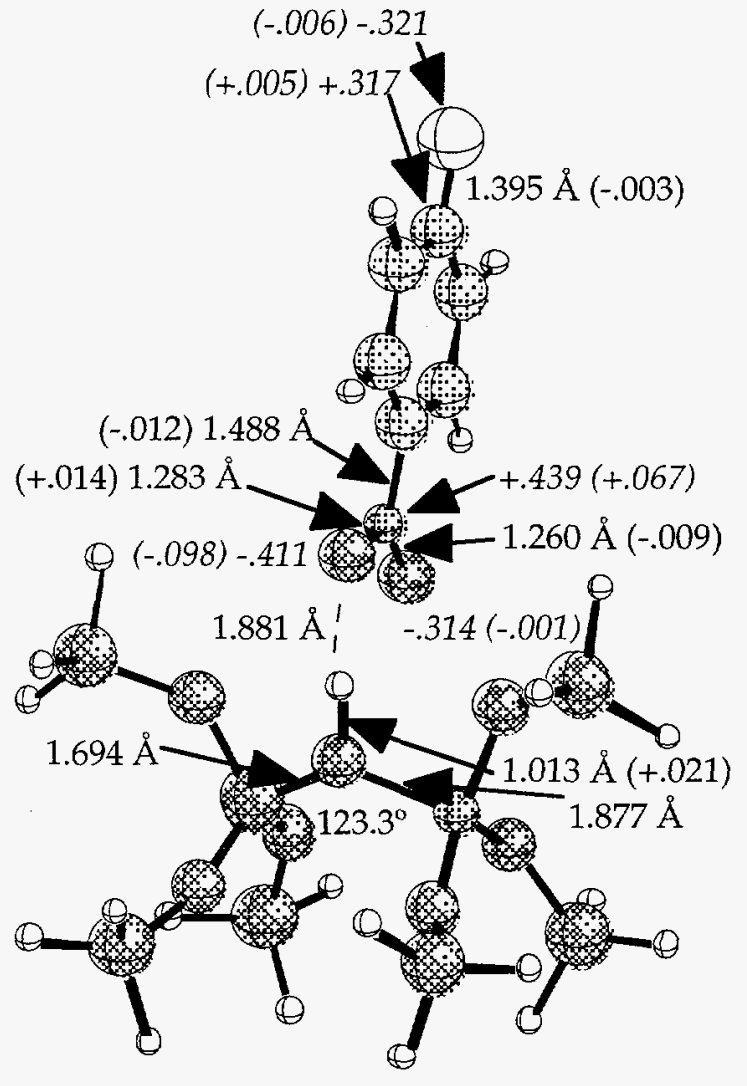

Figure 2.2. Optimized geometry for the p-fluoronitrobenzene-zeolite complex optimized at the BLYP/DNP level of theory. Partial charges (italics) and internal coordinates for the optimized complex are listed on the figure. Values in parentheses are the difference between the optimized values in the complex and those of the isolated neutral adsorbate and the isolated neutral zeolite. The energy of adsorption is $7.7 \mathrm{kcal} / \mathrm{mol}$ for this model.

DFT Study of the Protonation of Alkenes by Zeolites (Q. Zhao ${ }^{(\alpha)}$ and J. B. Nicholas)

(a) AWU Postdoctoral Research Associate

The protonation of alkenes in zeolites is the first step in many hydrocarbon reactions. In strong liquid acids, this reaction involves the creation of carbenium ion intermediates, which are stabilized by the large dielectric effects of the liquid. There is an increasing amount of evidence which indicates that zeolites are not capable of stabilizing most carbenium ions within the zeolite channels. Rather, the zeolite catalyzes proton addition 
by a mechanism that involves a carbenium-like transition state, avoiding carbenium intermediates. The lack of carbenium ion intermediates has profound implications regarding the strength of zeolite acidity and the nature of zeolite reactions. This is particularly important when we consider that much of what has been written about zeolite reaction mechanisms assumes carbenium intermediates, despite the fact that there is no experimental evidence for the existence of such species.

In Figure 2.3 we show the transition state for zeolite proton addition to ethylene, propylene, and isobutene. In each case the breaking of the zeolite-proton bond is accompanied by the creation of a bond between the alkene carbon and the zeolite oxygen, resulting in a surface alkoxide species. The extent of proton transfer, as measured by the lengths of the O-H-C bonds, parallels the expected stability of the carbenium-

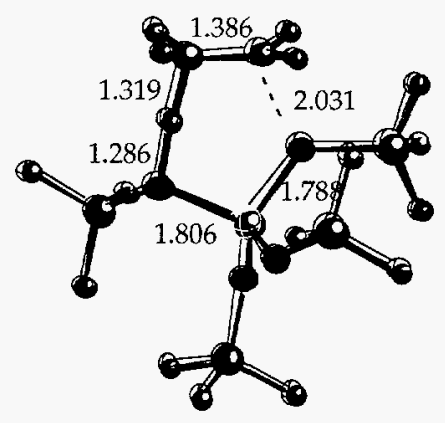

Ethylene

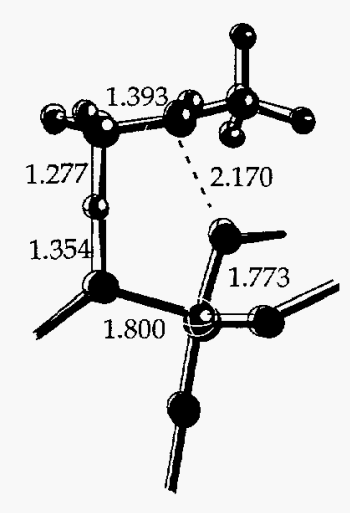

Propylene

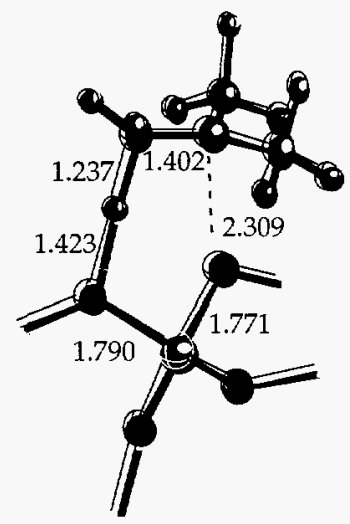

Isobutene
Figure 2.3. Optimized transition states for protonation of ethylene, propylene, and isobutene by a zeolite. Calculations at the SVWN/DNP level of theory. like transition state (ethylene is primary, propylene is secondary, and isobutene is tertiary). No carbenium intermediates are theoretically predicted in this reaction, agreeing with their lack of detection by experimental methods.

NMR and DFT Study of H-D Exchange in Benzene on Acidic Zeolites (L.W. Beck, ${ }^{(a)}$ J. B. Nicholas, and J. F. Haw ${ }^{(a)}$ )

(a) Laboratory for Magnetic Resonance and Molecular Science, Department of Chemistry, Texas A\&M University

The exchange between protons of acidic zeolites and adsorbed hydrocarbons is a facile process that provides fundamental information on zeolite acidity and mechanism. This exchange can be measured experimentally using deuterium labeled adsorbates and is also ideal for theoretical study. However, whereas both theory and experiment can provide information about the rate of proton $\mathrm{H}-\mathrm{D}$ exchange, only theory at present can determine the transition state for the reaction. The accepted mechanism for $\mathrm{H}-\mathrm{D}$ exchange in liquid acid involves the formation of a stable $\sigma$-complex carbenium intermediate. As we have discussed previously, the zeolite lacks the ability to stabilize such ionic intermediates, and the reaction proceeds by alternate pathways.

In Figure 2.4 we show the transition state for $\mathrm{H}-\mathrm{D}$ exchange of benzene, calculated at the BLYP/DNP level of theory. Note that the transition state is a carbenium like $\sigma$-complex, but no stable carbenium intermediate need be involved. Thus, the zeolite reaction mechanism is closely related to that for the protonation of alkenes presented earlier, and does not follow the pathway expected for liquid acids. The theoretical benzene H-D transition state is analogous to the carbonium-like state presented by van Santen and coworkers for methane H-D exchange on zeolites. ${ }^{22}$

\section{References}

D. E. Perry, Surf. Sci. 49, 433 (1975).

2 J. Perez-Jorda, A. Becke, and E. San-Fabian, J. Chem. Phys. 100 (1994).

3 J. E. Jaffe, N. M. Harrison, and A. C. Hess, Phys. Rev. B 49, 11153 (1994). 


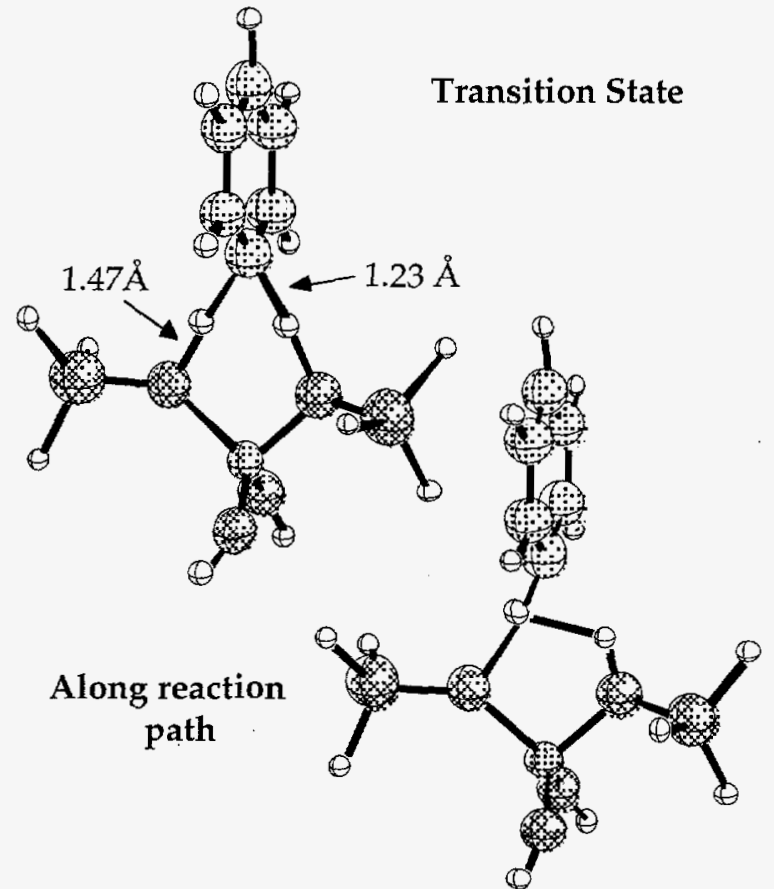

Figure 2.4. The transition state for $H-D$ exchange in the benzene/zeolite complex, optimized at the BLYP/DNP level of theory. Also shown is a step along the reaction path between the reactants and the transition state.

E. I. Solomon, P. M. Jones, and J. A. May, Chem. Rev. 93, 2623 (1993).

Imanishi, Nobuyuki, Toyoda, Mie, Takeda, Yasuo, Yamamoto, and Osamu, Solid State Ionics 58, 333 (1992),

6 C. Julien, T. Sekine, and M. Balkanshi, Solid State Ionics 48, 225 (1991).

7 M. J. McKelvy and W. S. Glaunsinger, J. Sol. State Chem. 67, 142 (1987).

Fast Ion Transport in Solids, Vol. 250, edited by B. Scrosati, A. Magistris, C. M. Mari, and Mariotto (1993).

9 R. Dovesi, V. R. Saunders, and C. Roetti, (Universit à di Torino, Torino, Italy, 1992).

10 C. Pisani, R. Dovesi, and C. Roetti, Hartree-Fock Ab Initio Treatment of Crystalline Systems (Springer-Verlag, New York, 1988).

11 C. N. R. Rao and Gopalakrishnan, New Directions in Solid State Chemistry: Structure, Synthesis, Properties, Reactivity and Materials Design (University Press, Cambridge, New York, 1986).

12 M. S. Stave and J. B. Nicholas, J. Phys. Chem. 97, 630 (1993).

13 M. S. Stave and J. B. Nicholas, in Theoretical and Computational Approaches to Interface Phenomena, edited by H. Sellers and J. R. Golab (Plenum, New York, 1994), pp. 219.

14 C. T.-W. Chu and C. D. Chang, J. Phys. Chem. 89, 1569 (1985).
B. Delley, J. Chem. Phys. 92, 508 (1990)

B. Delley and D. E. Ellis, J. Chem. Phys. 76, 1949 (1982).

S. H. Vosko, L. Wilk, and M. Nusair, Can. J. Phys. 58, 1200 (1980).

A. D. Becke and R. M. Dickson, J. Chem. Phys. 89, 2993 (1988).

C. Lee, W. Yang, and R. G. Parr, Phys. Rev. B 37, 785 (1988).

A. D. Becke, J. Chem. Phys. 98, 5648 (1993).

C. Møller and M. S. Plesset, Phys. Rev. 46, 618 (1934).

G. J. Kramer, R. A. van Santen, C. A. Emeis, and A. K. Nowak, Nature 363, 529 (1993). 



\section{Biomolecular Modeling and Simulation}

Theory, Modeling, and Simulation

In 1994-95, Biomolecular Modeling and Simulation activities focused on applying existing modeling/simulation methods and developing improved computational methods to coupled theory-experimental studies, in order to uncover structure-function-dynamic relations of proteins/enzymes with an emphasis on biodegradative enzymes. The primary focus is to design new and improved catalytic functionality and/or alternative molecular properties of enzymes with an emphasis on developing near-, mid- and longterm strategies specific for biodehalogenation of contaminated deep subsurface DOE-sites. Two additional protein/enzyme areas of research for which work began within the last year are the areas of 'extremozymes' and protein-DNA complexes.

In a 1992 report of contaminants measured at 91 waste sites at $18 \mathrm{U}$. S. Department of Energy (DOE) facilities within the weapons complex, halogenated hydrocarbon solvents were found to be one of the most prevalent contaminant classes. Multi-halogenated ethylenes and ethanes were among the most prevalent and highest concentrated contaminants of this class occurring in ground water and soils/sediments. ${ }^{1}$ Compounds in this class have been observed in ground water and/or sediment to reach concentrations in excess of two million times U. S. Environmental Protection Agency guidelines. In addition to problems at DOE sites, many other government facilities as well as industrial sites have similar serious problems with halogenated solvents. The nature of deep contamination (commonly dilute, highly dispersed, and inaccessible) limits or precludes the application of conventional engineering strategies that are currently being applied to remediate surface and near-surface contamination. Cost-effective remediation of heavily halogenated hydrocarbons in deep subsurface environments is for the most part limited to novel bioremediation alternatives. ${ }^{2}$ Although microorganisms have tremendous capacity to evolve to meet new selection pressures, the time scale required to do so, depending on the complexity of the required underlying molecular modifications, may be unacceptably long as measured by human time scales when hazardous, synthetic, recalcitrant substances are involved. 3,4

Rational enzyme redesign has the unique ability to foster discovery of new enzymatic forms that would otherwise have an extremely low probability of evolving biologically under either laboratory-controlled or natural conditions (which is to be expected for an enzyme requiring several simultaneous and specific amino acid changes in order to achieve the new functionality). To have a reasonable chance to successfully carry out rational enzyme redesign to alter specificity, improve efficiency, or change the optimum range of functionality, certain enzyme prerequisites are essential. For instance, it is necessary to have adequate enzyme structural data, an understanding of the mechanism(s) of action, and a cloned gene. Such information is available for the P450cam variant of the ubiquitous biodegrading and detoxifying superfamily of enzymes known as cytochromes $\mathrm{P} 450$. In addition, such information is rapidly growing for other cytochromes P450, such as P450BM3, and dehalogenases.

Although experimentally determined enzyme structures are invaluable for understanding structure-function relations, the structural image obtained is an average in time and space. Nonaverage structural conformers, not resolvable by experimental methods, are also important since bioactivity is not necessarily explainable from a consideration of an average structure. Many

The work reported in this section was supported by the Health Effects and Life Sciences Research Division, Office of Health and Environmental Research in the $U$. S. Department of Energy and by Laboratory Directed Research and Development funds. Grants of computer time were provided at the National Energy Research Supercomputing Center by the Office of Scientific Computing in the U. S. Department of Energy. 
enzymes are thought to only transiently adopt functionally critical conformations; these transient conformations are not normally expected to be "visible" by high-resolution experimental methods. Computer simulations, starting from a high-resolution experimental structure, are capable of uncovering such structures. Experiments can then be designed to refute or confirm the significance of each transient population, in order to establish its role in bioactivity. Such collaborative coupled experiment-theory studies on cytochrome P450cam are under way in the laboratory of Professor Steve G. Sligar at the University of Illinois/Urbana and Professor Lawrence P. Wackett at the University of Minnesota/St. Paul. Similar coupled studies on haloalkane dehalogenase ${ }^{5}$ are under way with Professor Richard B. Janssen at the University of Groningen/Holland and with collaborators at PNNL for a newly isolated dehalogenase. In addition, simulations are being used to examine the extent of structural and dynamic relaxation within an enzyme when crystal packing or aggregation (in concentrated solution) interactions are no longer present; such relaxation perturbations may be essential to understanding function and for rational redesign.

In 1994 we initiated a related project: modeling and simulation of extremozymes. Some organisms are adapted to grow under extreme physical (temperature, pressure) and/or chemical (salt concentration, $\mathrm{pH}$, concentration of nonaqueous solvent) conditions. The accumulation of information such as sequence and structure for proteins and enzymes that function under extreme conditions is in the early stages. Understanding the basis for the sequence and structural changes will have many practical biotechnological implications because of the added experimental range of usefulness of these enzymes. The primary purpose of this task is to study the underlying structure-function-dynamic relations of proteins that function under normal physiological conditions and those that function under extreme conditions. Once a fundamental understanding is achieved, we shall be positioned to rationally redesign enzymes with a desired catalytic function, and in one or more extreme conditions, for instance, an extracellular dehalogenase that could function in concentrated halocarbon solvent. We are also interested in preserving enzyme functionality at unusual $\mathrm{pH}$, temperature, salt conditions, and combinations of the above.
In late 1994, we began coupled experimenttheory studies to understand the role of dynamics in protein-DNA recognition and complexation. with Professor Steve K. Burley of Rockefeller University in New York City. High quality experimental three-dimensional structures of protein-DNA complexes were used as the starting point for molecular dynamics (MD) simulations under in vivo solution conditions. Simulations were performed and analyzed on the complex and individual components of the complex, in order to understand the nature of recognition interactions. For instance, what is the role of 'induced-fit', 'lock-and-key', or a combination of such recognition processes? A fundamental understanding of protein-DNA complexes impacts 1) molecular health risk assessment based on a fundamental understanding of DNA repair enzymes, and 2) biotechnological applications associated with transcriptional regulation and improved precision in identifying and isolating unique sequences in the human genome. This latter area of research will not be further discussed in this report.

\section{Understanding Enzyme Structure-Function-Dynamics Relations}

Domain-Domain Dynamics: T4 Lysozyme (G. E. Arnold, J. I. Manchester, ${ }^{(a)}$ B. D. Townsend, ${ }^{(b)}$ and R. L. Ornstein)

(a) AWU Graduate Research Associate

(b) Department of Biophysics, The Johns Hopkins University, Baltimore

Domain-domain communication may play a key role in the functional energetics of enzymes. Such interactions can result in hinge-bending like motions, where relative changes in domain orientation allow for a continuum of protein conformational states, with only minor structural changes in the domains themselves. These types of dynamic conformational changes are a part of the enzyme's fundamental fluctuations and play an important role in substrate recognition and catalysis. Computer simulation of atomic motions provides an ideal method for studying atomic fluctuations which lead to concerted internal motions in proteins. Phage T4 lysozyme, a monomeric enzyme composed of 164 amino acids with an active site cleft situated between two 
globular domains, is an ideal system for studying such processes because of its structure.

Analyses of protein time-correlated atomic motions is one approach to elucidating such processes. In this method, protein time-correlated atomic motions were analyzed using a dynamical cross-correlation map (DCCM), which was calculated using the expression:

$$
C_{i j}=\left\langle\Delta r_{i} \bullet \Delta r_{j}\right\rangle /\left(\left\langle\Delta r_{i}^{2}\right\rangle\left\langle\Delta r_{j}^{2}\right\rangle\right)^{1 / 2}
$$

where $\Delta r_{i}$ is the displacement from the mean position of the $i$ th atom. The $\mathrm{C}_{\mathrm{ij}}$ elements of the matrix are symmetric about the diagonal; however, for clarity, only negative correlation values are displayed in the upper triangle and only positive correlation values are displayed in the lower triangle. Positive and negative $C_{i j}$ values result from atomic motions in the same and opposite direction, respectively, along a given spatial coordinate. Prominent in Figure 3.1 are two large blocks of positive correlation corresponding to the N-terminal lobe (12-59) and C-terminal lobe (81-160). These data demonstrate that atomic motions in these regions are cooperative and that these areas move as single units. Significant positive correlations are also found between residues
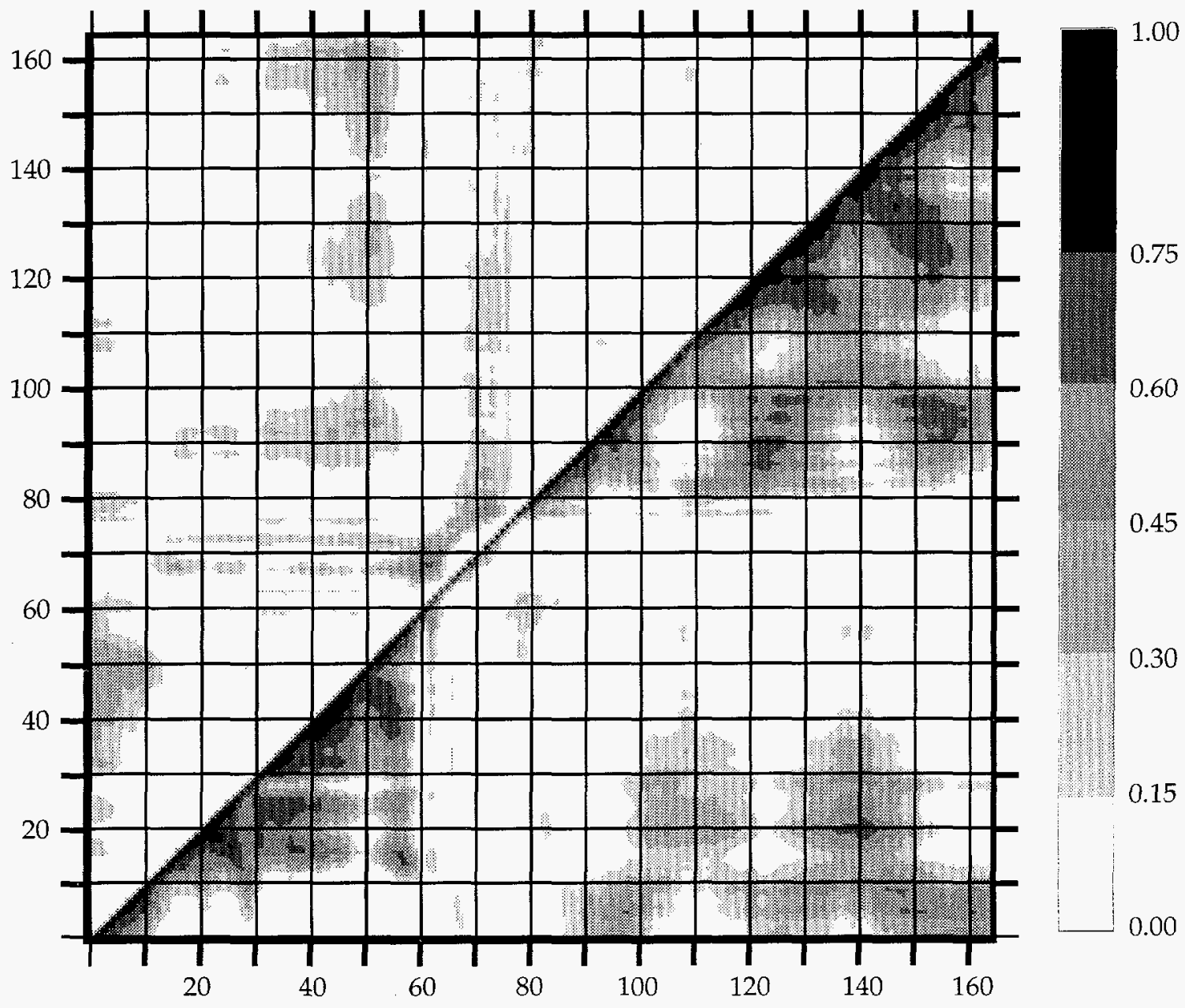

\section{RESIDUE NUMBER}

Figure 3.1. DCCM matrix calculated from the lysozyme trajectory. The spatial position of an individual amino acid was determined by calculating the geometric mean of its respective $N, C_{\alpha^{\prime}}$ and $C$ backbone atom positions. Due to the scalar product in the numerator of the DCCM equation, pair wise atomic motions whose directions are orthogonal with respect to each other will result in $C_{i j}=0$. 
2-11 and 85-160, indicating that residues in the A helix move in concert with those of the rest of the C-terminal domain. Also evident are off diagonal positive correlations (but to a lesser degree than those found in the two domains) between residues $\sim 17-35$ and $\sim 101-111$, and $\sim 17-35$ and $\sim 135-145$. The large positive correlated atomic motions found between residues 19-22 and 142 is especially noteworthy. These residues are located at the mouth of the active site and also around the active site proper, indicating some degree of communication between residues surrounding the catalytic cleft. Minor negative cross peaks are also detected in the upper left quadrant of the map between residues $\{32-59$ and $90-105\}$, \{32-59 and 122-137\}, and $\{32-59$ and $150-164\}$ diagnostic of a hinge-like motion. ${ }^{6}$

Crystal Packing Forces and Their Effects on Protein Conformation and Dynamics: T4 Lysozyme (G. E. Arnold and R. L. Ornstein)

In terms of protein structure, T4 lysozyme is one of the best studied enzymes with many dozens of crystal structures of both the native and mutant forms of the protein available in the Brookhaven Protein Data Base (determined by Professor Brian Matthews, University of Oregon, Eugene). The crystal structure of the native protein reveals that the enzyme active site is occluded, preventing substrate access to the binding pocket. In order for the enzyme to facilitate substrate binding, some sort of conformational flexibility must exist between the two enzyme domains. A T4 lysozyme mutant, M6I, has been crystallized in two different forms. ${ }^{7}$ The form I crystal is nearly isomorphous with the wild type crystal and has an active site mouth separation of $\sim 17.4 \AA$, as determined by the alpha-carbons distance between residues 35 and 141 . In the form II crystal, four distinct protein conformations are observed in the unit cell, and each protein structure has unique active site mouth separations ranging from $18.4 \AA$ to $25.2 \AA$. This wide range of active site separations raises an interesting question; are the displacements in the M6I crystal indicative of the range of motion for the enzyme in solution, or are the separations a result of crystal packing stabilization? To address this question we have performed three different 500 picoseconds ( $\mathrm{ps}=$ $10^{-12} \mathrm{~s}$ ) MD simulations that include the native enzyme and M6I mutant structures with active site displacements of 18.4 and $25.2 \AA .{ }^{8}$ Each simulation was performed with a $10 \AA$ explicit shell of water, all hydrogens, and use of dual non-bonded cutoff distances of 11.5 and $15.0 \AA$.

The simulations of native $\mathrm{T} 4$ and form I of the M6I mutant showed relatively minor conformational perturbations with respect to the incipient crystal structure. On average, the range of observed active site displacements for the two simulated proteins was $\sim 18 \AA$ with a fluctuation of $3 \AA$, as shown in Figure 3.2 (on the left). However, the trajectory of the more "open" mutant (form II) indicated that significant changes in relative domain orientation had occurred. We observed a gradual change in the active site displacement of this structure over the period of the trajectory, with the active site mouth separation decreasing from its initial value of $\sim 25 \AA$ to a final value of $\sim 17 \AA$, as shown in Figure 3.2 (on the right). The $\mathrm{MD}$ trajectories indicate that form II of the mutant closes and has a time-averaged structure (calculated over the final 50 ps of the trajectory) more similar to the wild type crystal structure than to its own starting crystal structure (as measured by backbone atom RMSD). These results suggest that crystal packing forces stabilize the mutant in its most "open" conformation, and that this "open" configuration is a relatively high energy enzyme conformation. The data also suggest that the range of motion for the enzyme in solution is on the order of $18 \pm 3 \AA$. Discussions are under way with the Matthews group to combine experiment and simulations, in order to better understand the relationship between the observed range of active site displacements observed in the crystal compared to in solution and how these relate to function.

Unexpectedly Large Dynamic Motions Across the 'Mouth' of the Active Site of Cytochrome P450BM3 (M. D. Paulsen, T. B. Nguyen, ${ }^{(a)}$ and R. L. Ornstein)

(a) AWU Postdoctoral Research Associate

While for some proteins, such as cytochrome P450cam, substrates and inhibitors bind to what appears to be an essentially rigid active site, for many other enzymes, binding of substrates or inhibitors induces significant conformational changes. In some cases, the relevant motion is a 

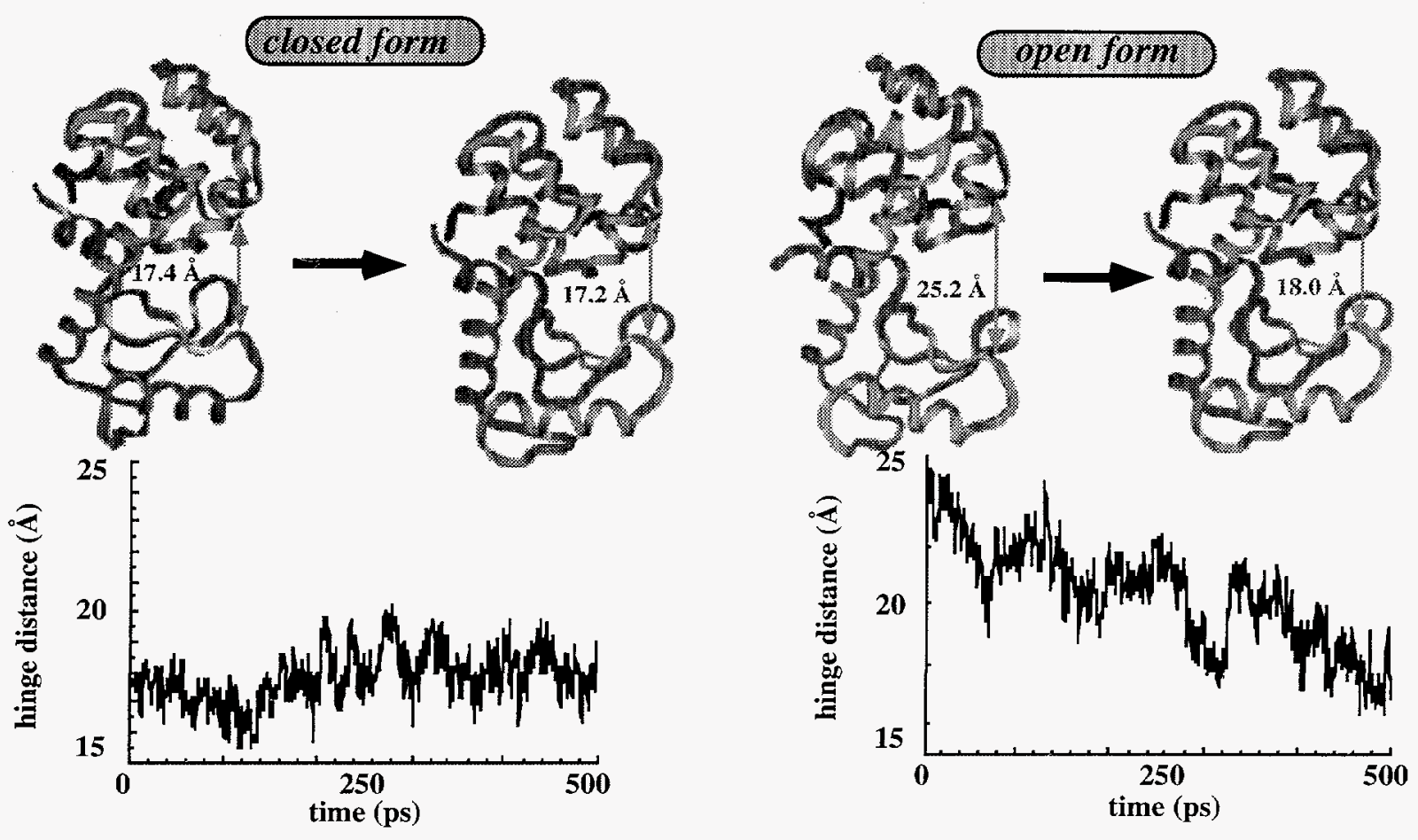

Figure 3.2. Displacements measured across the active site mouth between the $\alpha$-carbons of residues 35 and 141 as a function of time during the simulation. The active site displacement of the "open" form mutant approaches that of the native enzyme toward the end of the simulation.

hinge-bending which leads to large-scale rigid body displacement of entire domains. Cytochrome P450BM3 from Bacillus megaterium catalyzes the hydroxylation of 14 - to 20 -carbon saturated and unsaturated fatty acids; the 471 amino acid heme domain has been crystallized and a $2.0 \AA$ resolution structure reported. ${ }^{9}$ Interestingly, the asymmetric unit cell contains two enzyme molecules which differ in their conformation around the substrate binding pocket. Molecule 2 in the asymmetric unit has a more open and accessible substrate binding pocket than molecule 1. The difference in conformation is described as rigid body rotations of the $\mathrm{N}$ terminal domain and the F-G domain relative to the main body of the enzyme. In this sense the crystal structure of $\mathrm{P} 450 \mathrm{BM} 3$ is reminiscent of the recently reported structures of the M6I mutant of T4 lysozyme which had five different allomorphs in the crystal related to each other by rigid body displacements of the $\mathrm{N}$ - and C-terminal domains. ${ }^{7}$ Both the more open conformation of molecule 2 of the P450BM3 crystal and the more closed active site of molecule 1 appear to be stabilized by a number of crystal contacts. Thus, it is unclear whether the two conformations reported by the crystallographers would be highly populated in a noncrystalline environment. In the absence of these crystal contacts, this functionally important region of P450BM3 could possibly adopt still other conformations than the ones observed in the crystal structure.

Molecular dynamics trajectories were calculated separately for each of the two molecules in the asymmetric unit of the crystal structure of the hemoprotein domain of cytochrome P450BM3. ${ }^{10}$ Each simulation was 200 ps in length and included a $10 \AA$ layer of explicit solvent. The simulated time-average structure of each P450BM3 molecule is closer to its crystal structure than the two molecular dynamics time-averaged structures are to each other. In the crystal structure, molecule 2 has a more accessible substrate binding pocket than molecule 1 , and this difference is maintained throughout the simulations presented here. In particular, the substrate docking region (i.e., the 'mouth' of the active site) of molecule 1 and molecule 2 diverge in the solution state simulations. The mouth of the substrate binding 
pocket, defined between the C-alpha carbons of Pro-45 and Ala-191, is significantly more mobile in the simulation of molecule 2 than in the simulation of molecule 1 (Figure 3.3). For molecule 1, the width of the mouth is only slightly larger than its $x$-ray value of $8.7 \AA$ and undergoes fluctuations of about $1 \AA$. However, in molecule 2 , the mouth of the substrate binding pocket is dramatically more open in the time-average molecular dynamics structure $(14.7 \AA)$ than in the $x$-ray structure $(10.9 \AA)$. Furthermore, this region of the protein undergoes large amplitude motions during the trajectory that are not seen in the trajectory of molecule 1 ; repeatedly opening and closing up to $7 \AA$. Presumably, the binding of different substrates will induce the mouth region to adopt different conformations from within the wide range of structures that are accessible. Additional studies are in progress testing the sensitivity of the large observed mouth opening to variations in the simulation protocol used.

\section{Understanding and Improving Enzyme Dehalogenation}

Dehalogenation of Pentachloroethane by Cytochrome P450cam: Why F87W Is Faster than Wild-Type (J. I. Manchester ${ }^{(a)}$ and R. L. Ornstein)

(a) AWU Graduate Research Associate

Cytochrome P450cam catalyzes the reductive dehalogenation of several halogenated hydrocarbons at rates approximately two orders of magnitude more slowly than it hydroxylates camphor. ${ }^{11}$ Recently, Loida ${ }^{12}$ observed that a single active site mutation, substituting tryptophan for phenylalanine at position 87 (F87W), increased the rate of pentachloroethane (PCA) dehalogenation threefold. To gain insight into the effects of tryptophan substitution on a molecular level, we performed 200 ps MD simulations on both wild-type and mutant P450cam enzymes with PCA bound in the active site pocket. We found that tryptophan in the

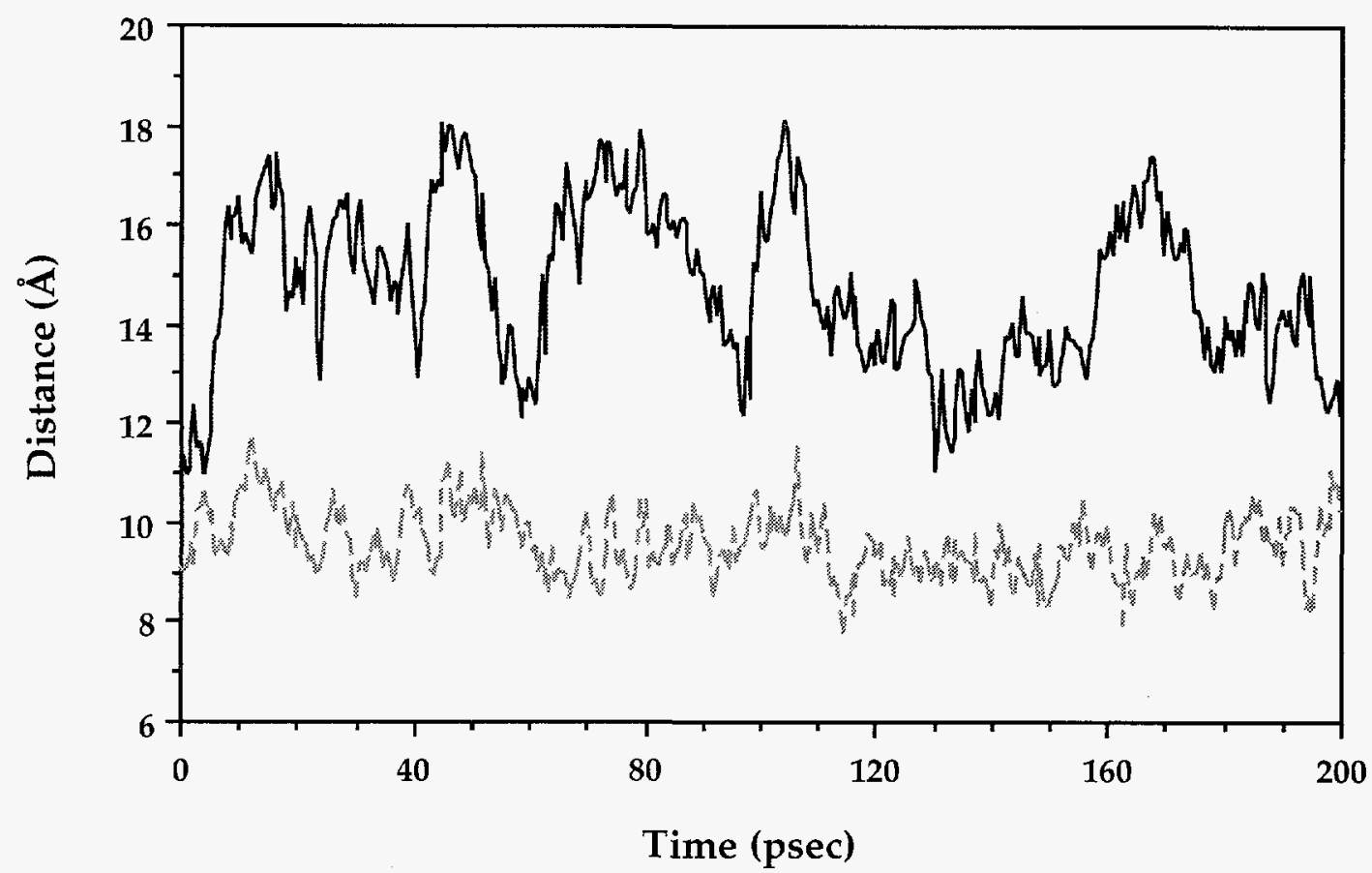

Figure 3.3. The distance between the $\mathrm{C}$-alpha carbons of residues 45 and 191 in cytochrome $\mathrm{P} 450 \mathrm{BM} 3$ is plotted as a function of time of the simulation for molecule 1 (lower trace) and molecule 2 (upper trace). 
mutant sterically limits the configurational freedom of the substrate to a region close to the heme-Fe. This effect can be seen from the difference in the time-averaged position of the substrate in either simulation (Figure 3.4). Over the final 150 ps of dynamics, PCA contacts the heme-Fe twice as often in the mutant as it does in wild-type P450cam. These results demonstrate that significantly faster rates of dehalogenation may be achieved simply by improving the proximity of a substrate to the heme-Fe. ${ }^{13}$

Substantial rate enhancement may be observed in P450cam variants that also improve the substrate reduction potential. Coupling close proximity with a favorable redox environment is the major thrust of our current redesign efforts. Novel mutant enzymes, engineered to incorporate both factors in the active site, are currently being evaluated using MD simulations.

Toward Rational Redesign of Haloalkane Dehalogenase (G. E. Arnold and R. L. Ornstein)

Haloalkane dehalogenase, from Xanthobacter autotrophicus, converts 1-haloalkanes to their corresponding alcohols with concomitant release of halide ion. Several crystal structures of the 310 amino acid enzyme have been solved, and atomic coordinates have been resolved for enzyme structures with bound substrate, without substrate, and with the trapped covalent ester intermediate. ${ }^{5}$ The reaction appears to be a two-step process in which, 1) substrate initially undergoes nucleophilic attack by an aspartic acid residue, resulting in a covalent ester intermediate, followed by, 2) hydrolysis of the ester by a water molecule. Unfortunately, the enzyme's turnover number for this reaction is quite low $\left(\mathrm{k}_{\text {cat }}=6 \mathrm{~s}^{-1}\right)$, and the measured enzyme affinity for substrate is also not very large $\left(\mathrm{K}_{\mathrm{m}}=1.1 \mathrm{mM}\right)$. The active site geometry is such that these key residues are ideally positioned for catalysis, so it has been suggested that it is not the catalytic active site chemistry that is the cause of low enzyme efficiency, but rather, access of substrate to the active site and/or release of product from the active site. Consequently, in order for haloalkane dehalogenase to become an effective bioremediatory enzyme, its catalytic efficiency must first be improved. Toward this goal, we have undertaken an effort to rationally redesign the haloalkane dehalogenase enzyme.

To better understand the underlying dynamics operative in controlling the substrate/product entrance and exit channels, we have recently
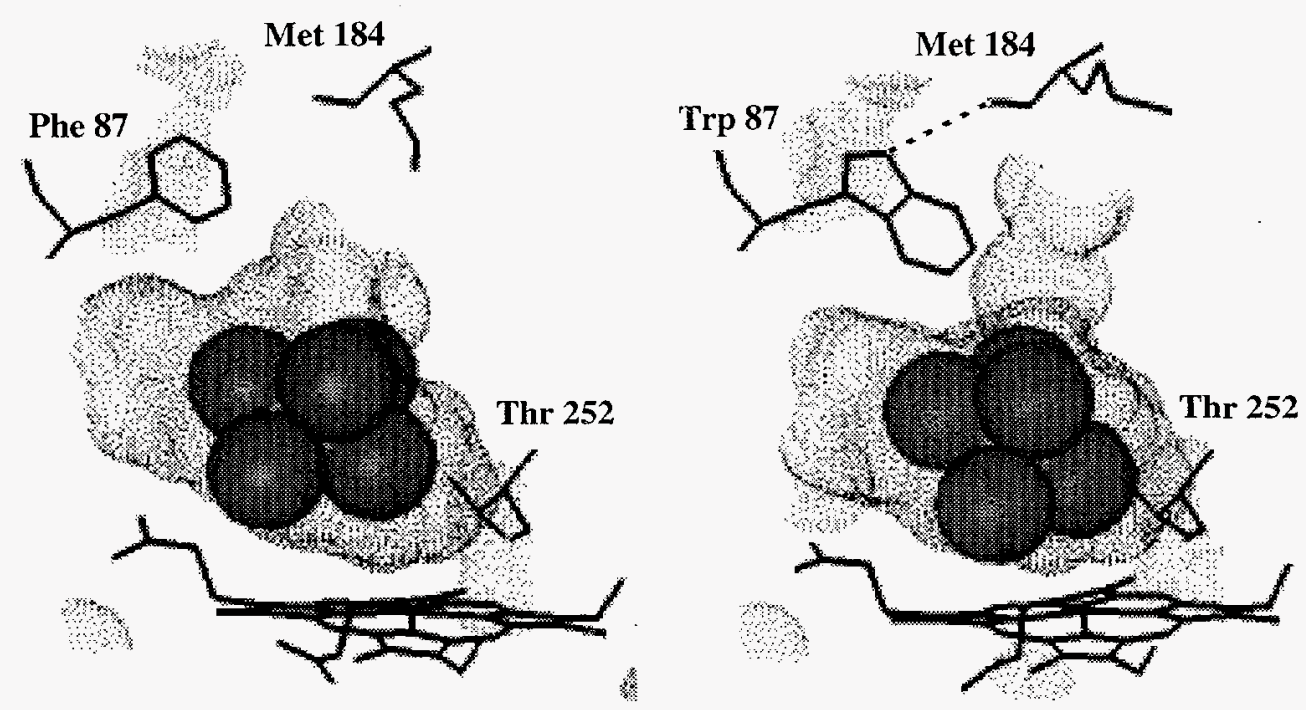

Figure 3.4. Solvent-accessible dot surfaces represent the active site of P450cam as it is seen by PCA, represented here by solid spheres. The F87W-P450cam binding pocket (left) is smaller than the wild-type's (right), forcing the substrate, on average, closer to the heme (bottom of view). Since the hydrogen bond donor of Trp 87 interacts strongly with Met 184 (dashed line, top right), it is mainly more frequent hemesubstrate interactions that give the mutant enzyme its faster dehalogenating properties. 
completed several 300 ps MD simulations of haloalkane dehalogenase using crystal structures of 1) substrate free enzyme, 2) substrate bound enzyme, and 3) the trapped covalent intermediate as starting structures. Each simulation was performed with a $10 \AA$ explicit shell of water, all hydrogens, and use of dual non-bonded cutoff distances of 11.5 and $15.0 \AA$. A complete analysis is under way with a primary goal of identifying key residues governing the active site mechanics which facilitate access to the binding pocket. Based on these findings, we will propose new mutant enzyme structures to be made and tested by our collaborators (Professor Dick B. Janssen, University of Groningen, Holland) with the intent of improving overall enzyme efficiency.

In addition, because neither molecular oxygen nor metallic enzyme cofactors are needed for enzymatic catalysis of the reaction, this enzyme is an especially good target for secondary redesign to improve its stability and functional range beyond normal cytoplasmic conditions in living bacteria. Thus, we have targeted this system and other similar systems for the future design of 'extremozymes', with improved dehalogenating activity. The new extremozyme work will be discussed in a later section.

\section{Methods Validation and Improvement for Enzyme Simulations}

Prediction and Experimental Verification of Product Profiles: Cytochrome P450cam Hydroxylation of Norcamphor (M. D. Paulsen, G. E. Arnold, R. L. Ornstein, P. J. Loida, ${ }^{(a)}$ and S. G. Sligar ${ }^{(a)}$ )

(a) School of Chemical Sciences and The Beckman Institute for Advanced Science and Technology, University of Illinois at Urbana

Cytochrome P450cam has been the subject of several theoretical and experimental studies of protein-small molecule recognition. A number of these studies have focused on characterizing structure-function relationships in the enzymesubstrate complex by utilizing camphor analogs to probe specific features of the enzyme active site. In particular, several theoretical and experimental studies have used norcamphor, a smaller more spherical analog of camphor to characterize the contribution of hydrophobic contacts to the regiospecificity of the hydroxylation reaction and to the degree of coupling between NADH consumption and product formation. However, the interpretation of these experiments and their comparison with theoretical calculations is complicated by the fact that a racemic substrate was used in the experiments instead of the pure enantiomers. Thus the observed changes in regiospecificity of the hydroxylation reaction can not be unequivocally attributed to specific substrate-enzyme interactions. Recently, a successful chiral separation of (1R)- and (1S)norcamphor was achieved. The earlier characterization of racemic norcamphor with respect to the product profile, steady-state kinetics, coupling efficiency, and substrate binding affinity was repeated for the enantiomerically pure analog. With the exception of the product profile, all of the reaction and physical parameters were similar for each enantiomer of norcamphor. However, consistent with the theoretical prediction, the regiospecificity of hydroxylation was enantiomer specific. The racemic mixture had been previously reported to give essentially equimolar amounts of the 5- and 6- alcohol as products with the 3 -alcohol as a minor product. Our theoretical calculations predicted that (1R)norcamphor would be preferentially hydroxylated at the 5 position while (1S)-norcamphor would be preferentially hydroxylated at the 6 position (Figure 3.5). This trend was qualitatively the same whether the simulations treated the heme iron in a pre-reactive non-iron-oxo form ${ }^{14}$ or in a reactive (presumptive) iron-oxo form. ${ }^{15}$ The experimentally determined (1R)-norcamphor product profiles was 65:30:5 for 5-, 6-, and 3alcohols, respectively, while for (1S)-norcamphor the profile was 28:62:10 in good agreement with the theory. ${ }^{15}$

Extremozymes - Toward Improving Nonaqueous Functionality: Subtilisin (Y.-J. Zheng ${ }^{(a)}$ and R. L. Ornstein)

(a) AWU Postdoctoral Research Associate

Several advantages of carrying out enzymatic reactions in nonaqueous environments have been identified including higher solubility of nonpolar substrates in organic solvent, increased thermostability of enzymes, altered substrate specificity, enzyme recovery from organic solvent, and ability to carry out reactions which 


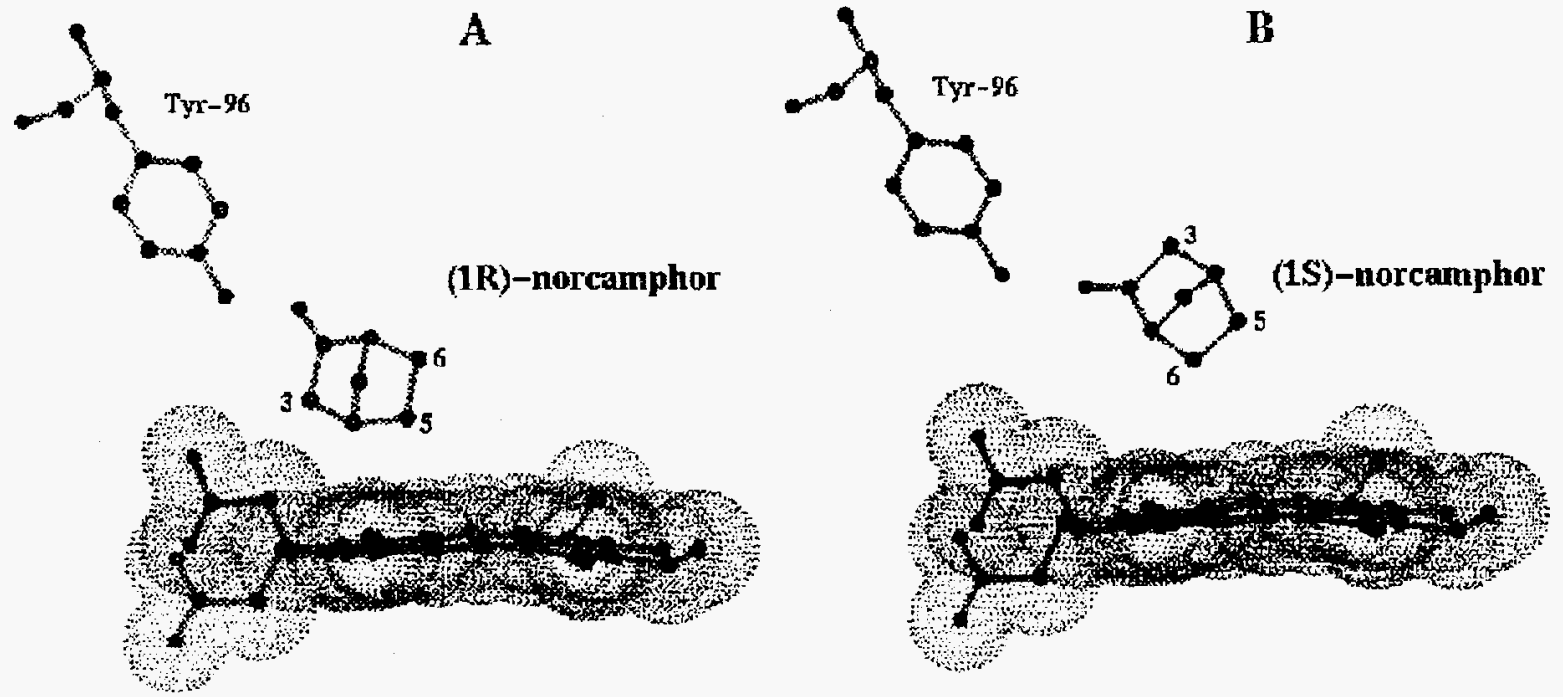

Figure 3.5. The active site of cytochrome P450cam with (1R)- and (1S)-norcamphor bound. For both substrates the hydrogen bond between the keto oxygen of the substrate and the sidechain of Tyr-96 is intact.

are impossible in water. In 1993, Fitzpatrick et al. ${ }^{16}$ solved the crystal structure of subtilisin Carlsberg in anhydrous acetonitrile and they found that the structure in anhydrous acetonitrile is essentially identical to the structure of the same enzyme in water. We recently performed two separate simulations: 1 ) in aqueous environment, and 2) in acetonitrile solution. Using AMBER 4.0 and the all-atom AMBER force field, ${ }^{17}$ subtilisin was solvated by a $35 \AA$ sphere of solvent molecules in both cases. A 510-ps simulation was carried out for subtilisin in acetonitrile and a 450ps simulation for subtilisin in water.

We have compared the calculated and the $x$-ray crystallographic temperature factors for subtilisin in water and acetonitrile. In aqueous environment, the match between the calculated and experimental temperature factors are very good. Some of the calculated peaks are higher than experimental, which is typical since our simulation was done in solution while the experimental temperature factors correspond to protein in crystal environment. However, the match in acetonitrile is not as good as in water. The calculated temperature factors are much higher than the x-ray crystallographic values. This discrepancy could be due to two factors: crystal contacts and cross linkers. Both will dampen atomic motions in the crystal. The protein atoms are much more mobile in acetonitrile than in water. This is in agreement with the observation that without cross linkers the crystal will shatter in acetonitrile. The calculated rms deviations from x-ray structures in both simulations are similar with the one in acetonitrile being slightly higher. Throughout both simulations the individual secondary structure elements (helices and sheets) remain intact. In the acetonitrile simulation, however, one of the helices undergoes a reversible helix unwinding motion, which lasts for about 100 ps (see Figure 3.6); we did not see such behavior in the aqueous environment. Work continues in order to better understand the structural and dynamic response of enzymes in various nonaqueous solvents so that we may rationally redesign targeted enzymes for functionality in specific solvents such as a deep subsurface plume of halogenated hydrocarbons. 


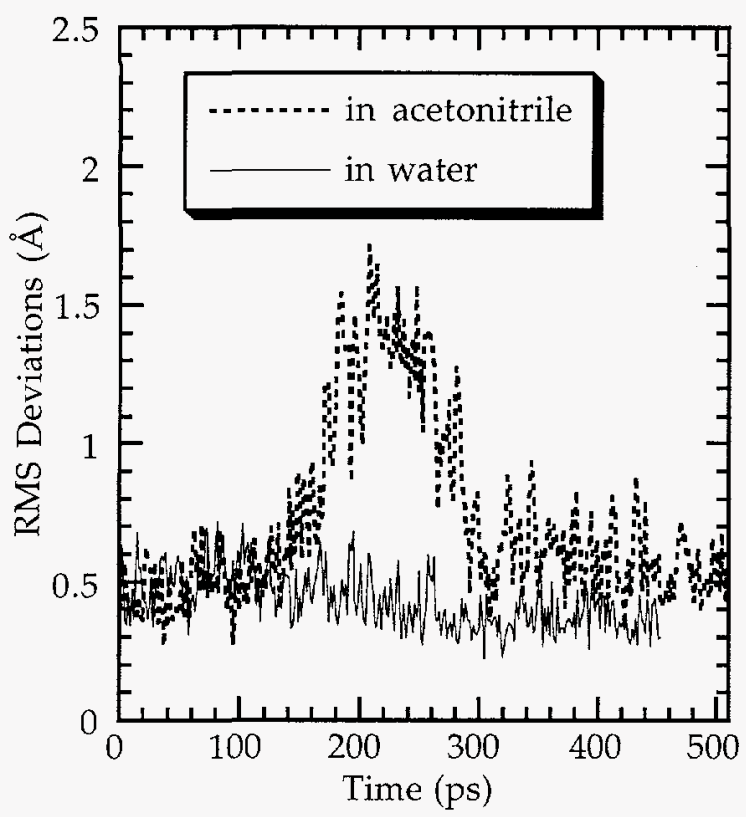

Figure 3.6 The calculated root-mean-square (RMS) deviations for helix 5 of subtilisin in water and acetonitrile.

Modeling a New Reductive Dehalogenase and Experimental Collaborations in EMSL (R. J. Martinez, ${ }^{(a)}$ L. Xun, ${ }^{(b)}$ M. A. Kennedy, ${ }_{r}^{(c)}$ and R. L. Ornstein)

(a) AWU Postdoctoral Research Associate

(b) Joint appointment with Earth and Environmental Sciences, PNNL, and Washington State University

(c) Macromolecular Structure and Dynamics, EMSL

Recently, a new enzyme was identified from the bacteria Flavobacterium sp., tetrachloro-phydroquinone reductive dehalogenase (TECH$\mathrm{RD})$, that catalyzes the dechlorination of tetrachlorohydroquinone as part of an enzymatic pathway for degrading pentachlorophenol, a widespread pollutant. A number of characterization studies were completed and the amino acid sequences of the enzyme was determined. To date no three-dimensional structural data is available which precluded detailed structure/function studies. The scarcity of enzymes capable of degrading polyhalogenated aromatics and the fact that TECH-RD is the only microbial dehalogenase currently available make it an important target for studying enzymatic structurefunction-dynamic relations.
Based on a comparison of amino acid sequence and enzymatic mechanism, TECH-RD appears to be functionally (and structurally) related to the family of glutathione-S transferases (GSTs). Crystal structures of five members of the GST family from three different families (mu, alpha, and pi) have been reported. All five share a similar structure based on eight alpha helices divided into two domains. These structures served as the basis for developing a three-dimensional model of TECH-RD. Multiple sequence alignment was used to determine which segments of TECH-RD correspond to the secondary structure elements in the known GSTs. Secondary structure prediction algorithms including Chou-Fasman and GOR were used to assess the reasonableness of the initial secondary structure assignments for TECH-RD. This information in conjunction with a distance geometry approach was used to obtain a family of potential structures for TECH-RD. Predictions that will serve to guide future sitedirected mutagenesis experiments have been made for the residues lining the glutathione binding site. Also, the model structures of TECH-RD will be useful in the early analysis of data from NMR experiments currently under way. Finally, an NMR-based structure, once determined will serve as an excellent test of the strengths and limitations of our modeling approach.

\section{References}

1

R. G. Riley, J. M. Zachara, and F. J. Wobber, (U. S. Department of Energy, Washington D.C., 1992).

2 F. J. Brockman and R. L. Ornstein, in Hazardous Materials Control/Superfund ' 91 (Materials Control Research Institute, Greenbelt, Maryland, 1991), pp. 264.

3 R. L. Ornstein, in On Site Bioreclamation: Processes for Xenobiotic and Hydrocarbon Treatment, edited by R. E. Hinchee and R. F. Olfenbuttel (Butterworth-Heinemann, Boston, Massachusetts, 1991), pp. 509.

4 R. L. Ornstein, in Structural Biology: State of the Art 1993, Vol. 1, edited by R. H. Sarma and M. H. Sarma (Adenine Press, Albany, New York, 1994), pp. 59.

5 K. H. G. Verschueren, F. Seljee, H. J. Rozeboom, K. H. Kalk, and B. W. Dijkstra, Nature 363, 693 (1993).

6 G. E. Arnold, J. I. Manchester, B. J. Townsend, and R. L. Ornstein, J. Biomol. Struct. Dyn. 12, 457 (1994).

7 H. R. Faber and B. W. Matthews, Nature 348, 263 (1990).

8 G. E. Arnold and R. L. Ornstein, Proc. Natl. Acad. Sci. USA, (submitted) (1995).

9 K. G. Ravichandran, S. S. Boddupalli, C. A. Haseman, J. A. Peterson, and J. Deisenhofer, Science 261, 731 (1993). 
10 M. D. Paulsen and R. L. Ornstein, Proteins, (in press) (1995).

11 S. Li and L. P. Wackett, Biochemistry 32, 9355 (1993).

12 P. L. Loida, Ph.D. with S. Sligar, University of Illinois, Urbana-Champaign, 1994.

13 J. I. Manchester and R. L. Ornstein, Prot. Eng. 8, 801 (1995).

14 M. B. Bass and R. L. Ornstein, J. Comp. Chem. 14, 541 (1993).

15 P. J. Loida, S. G. Sligar, M. D. Paulsen, G. E. Arnold, and R. L. Ornstein, J. Biol. Chem. 270, 5326 (1995).

16 P. A. Fitzpatrick, A. C. U. Steinmetz, D. Ringe, and A. M. Klibanov, Proc. Natl. Acad. Sci. USA 90, 8653 (1993).

17 S. J. Weiner, P. A. Kollman, D. A. Case, U. C. Singh, C. Ghio, G. Alagona, S. Profeta, and P. J. Weiner, J. Am. Chem. Soc. 106, 766 (1984). 

The mission of the High Performance Computational Chemistry (HPCC) group is to develop molecular modeling software applications for computers that provide $10-100$ times more computing capability than has been available with conventional vector supercomputers. While increases in raw computing power alone will greatly expand the range of problems that can be treated by theoretical chemistry methods, a significant investment in new algorithms is needed to fully exploit the potential of computers composed of massively parallel processors (MPPs). Merely porting presently available software to these parallel computers does not provide the efficiency required to exploit their full potential. Most existing parallel applications show a significant deterioration in performance as greater numbers of processors are used. In some cases, the efficiency is so poor that the use of additional processors decreases, rather than increases, the performance. Thus, new algorithms must be developed that exhibit parallel scalability (i.e., show a nearly linear increase in performance with the number of processors). Although perfect scalability is very difficult to achieve, we have demonstrated our ability to approach this level of performance with our new self-consistent field (SCF) codes (see below).

Another important consideration in using MPPs is how data are stored. So-called replicated-data schemes require that a copy of each data item in the program be stored on each processor, so that the size of the problem that can be handled is limited by the memory of a single processor. In distributed-data applications, each processor holds only a part of the total data; in such cases, the problem size is limited only by the total memory of the machine, allowing the treatment of much larger problems. Our efforts focus on distributed-data applications. They span virtually the whole range of computational chemistry methods, with significant efforts in Hartree-Fock and correlated techniques, multiconfiguration self-consistent field methods (MCSCF), second-order Møller-Plesset perturbation theory (MP2) (also known as second-order many-body perturbation theory) density functional theory (DFT), semiempirical methods, and classical Monte Carlo (MC) and molecular dynamics (MD).

In addition to the creation of new algorithms for computational chemistry on parallel processors, we are also creating the high-level data and control structures needed to make parallel programs easier to write, maintain, and extend. We have developed portable tools for memory allocation, message passing, distributed global arrays, performance analysis, decomposition strategies, prototyping of new kernels, refinement of performance models, code architecture determination, and teraflops projections. Those tools crucial to our parallel chemistry applications are considered in the first section below entitled Software Development Tools.

Our new computer codes feature major design improvements over traditional codes, are modular at all levels, and have culminated in the NorthWest Chemistry (NWChem) package. We consider issues central to the design, implementation, and performance of many of the component modules of NWChem in the second section which is entitled Parallel Computational Chemistry Applications.

Collaborations play a large role in defining and delivering the EMSL software. PNNL is one of the founding members of the Concurrent

The work reported in this section was supported by the Environmental and Molecular Sciences Laboratory Project, the U. S. Department of Energy's HighPerformance Computing and Communications Initiative, Laboratory Directed Research and Development funds, and a grant of computer time at the National Energy Research Supercomputer Center by the Office of Scientific Computing in the U.S. Department of Energy. 
Supercomputing Consortium (CSCC) and has a record of developing successful collaborations with other government agencies and industrial partners. The EMSL provides one of the key teams in DOE's Grand Challenge Software Project in High Performance Computational Chemistry, joint with Argonne National Laboratory. This project is developing new algorithms, software, and diagnostics required to fully exploit massively parallel computers capable of teraflops performance. The work focuses on a suite of computational chemistry modeling methods appropriate to three specific problem areas of urgent interest to the DOE and the chemical and petroleum industries: the chemistry of clay minerals, the chemistry of halohydrocarbons, and the rational redesign of biodegradative enzymes. This project involves participation of five industrial collaborators (Allied Signal Inc., Amoco Research Center, DuPont, Exxon Research and Engineering Co., and Phillips Research Center). EMSL has developed formal liaisons with key parallel computational chemistry software efforts worldwide, including those at the Engineering and Physical Sciences Research Council (EPSRC) Daresbury Laboratory (UK), and at the Universities of Torino and Vienna. The collaboration in molecular science software between Daresbury Laboratory and PNNL is highlighted below in the third section entitled Collaboration in Molecular Science Software: Daresbury Laboratory - Pacific Northwest National Laboratory.

A wide range of on-going collaborations in computational chemistry methods and applications include those with institutions in the U. S. and Europe. Many of these collaborations have been formalized through the PNNL Affiliate Staff Scientist (PASS) program. Results of a select few of these collaborations are highlighted below in the last section entitled Application of Developed Software to Prototypical or Model Problems in Chemistry.

\section{Software Development Tools}

We describe below those tools crucial to our parallel chemistry applications, providing an outline of the role, status, and capabilities of the theoretical chemistry group's message passing toolkit (TCGMSG) and corresponding attributes of the tools for memory allocation and distributed global arrays. The central role and development of parallel linear algebra routines is also considered and the concept of the runtime database, a repository of parameters and information for all subsequent application modules, is introduced.

Message Passing (R. J. Harrison and J. Nieplocha ${ }^{(\mathfrak{a})}$ )

(a) Computer and Information Science Department, PNNL

TCGMSG ${ }^{1}$ is a toolkit for writing portable parallel programs using a message passing model Supported are a variety of common UNIX workstations, mini-super and super computers, and heterogeneous networks of the these computer systems, along with true parallel computers such as the Intel iPSC, Delta and Paragon, the Kendall Square Research KSR-1/2, the IBM SP1/2, and the Cray T3D. Applications port between all of these environments without modification to the parallel constructs. TCGMSG has distinguished itself from other toolkits in its simplicity, high performance, and robustness. The limited functionality provided includes point-to-point communication, global operations and a simple load-balancing facility - all designed with chemical applications in mind. This toolkit is available in the public domain (from ftp.tcg.anl.gov), and is distributed with a set of example chemical applications.

The TCGMSG programming model and interface is modeled after Argonne's PARMACS. ${ }^{2}$ The message-passing interface (MPI) standard ${ }^{3}$ will soon obsolete the TCGMSG interface. However, TCGMSG has superior robustness and performance when compared with current portable MPI implementations. Also, the simple TCGMSG interface is readily implemented using MPI.

Memory Allocation (D. E. Bernholdt, ${ }^{(a)}$ R. J. Harrison, R. J. Littlefield, $(b)$ and G. S. Thomas $^{(b)}$ )

(a) AWU Postdoctoral Research Associate

(b) Applied Mathematics, EMSL

One of the key problems with high performance parallel supercomputers is managing the memory utilization on each node. Many computa- 
tional chemistry and other FORTRAN-based applications use statically allocated arrays. These are either multiple arrays with hard-wired dimensions that commonly require recompiling to change the problem size, or static arrays that are used as simple stacks throughout the entire application. The Memory Allocator (MA) developed for NWChem is a library of routines for use by $C$, FORTRAN, or mixed-language applications. C applications can benefit from MA instead of the ordinary malloc() and free() routines because of the extra features MA provides: heap and stack memory management, debugging and verification support (e.g., detecting memory leaks), usage statistics, and quantitative memory availability information. FORTRAN applications can take advantage of the same features, and may in fact require a library such as MA because dynamic memory allocation is not supported by all implementations of the language. The coexistence of heap and stack operations can benefit application design by tuning the memory allocation based on the specific application requirements. Once FORTRAN-90 is widely available, library tools such as MA may not be required; but they are currently essential for memory management in complex applications.

MA is designed to be portable across a variety of platforms and is currently supported on nearly a dozen different workstations and highperformance parallel computers. The current implementation utilizes a segment of memory obtained from the operating system upon initialization. The low end of the segment is managed as a heap and the high end of the segment as a stack. The $C$ language interface utilizes standard Cinclude mechanisms, and the FORTRAN interface needs only a simple "include file" preprocessing system such as that provided by $c p p$. The allocated memory segment of each block can be typed explicitly as any formal type available in FORTRAN or C.

\section{Global Arrays (R. J. Harrison and J. Nieplocha ${ }^{(a)}$ )}

(a) Computer and Information Science Department, EMSL

Emerging standards for parallel programming languages (notably High Performance FORTRAN, HPF-1) do not provide extensive support for multiple instruction multiple data
(MIMD) programming. ${ }^{4}$ The only truly portable MIMD programming model is message passing, for which a standard interface has been proposed recently. ${ }^{3}$ However, it is very difficult to develop applications with fully distributed data structures using the message-passing model. .56 The sharedmemory programming model offers increased flexibility and programming ease, but is less portable and provides less control over the interprocessor transfer cost. What is needed is support for one-sided asynchronous access to data structures (here limited to one- and twodimensional arrays) in the spirit of shared memory. With some effort, this can be provided portably; in return for this investment, we gain a much easier programming environment, speeding up code development and improving extensibility and maintainability. We also gain a significant performance enhancement from increased asynchronous execution of processes. ${ }^{8}$ Message passing forces processes to cooperate (e.g., by responding to requests for a particular datum). Inevitably, this involves waiting for a collaborating process to reach the same point in the algorithm; the wait time is only partially reduced by the use of complex buffering and asynchronous communication strategies. With a one-sided communication mechanism, where each process can access what it needs without the explicit participation of another process, all processes can operate independently. This approach eliminates unnecessary synchronization and leads naturally to the interleaving of computation and communication. Most programs contain multiple algorithms, some of which may naturally be task-parallel (e.g., Fock matrix construction), and others which may be efficiently and compactly expressed as data-parallel operations (e.g., evaluating the trace of a matrix product). Both types of parallelism must be efficiently supported. Requirements were considered for the self-consistent field algorithm discussed later in this chapter, and also for the parallel COLUMBUS configuration interaction program, ${ }^{9} \mathrm{MP} 2,{ }^{10}$ and parallel coupled-cluster methods. ${ }^{11}$ The needs of these methods led to the design and implementation of the Global Array toolkit ${ }^{7}$ to support one-sided asynchronous access to globally addressable distributed oneand two-dimensional arrays. 


\section{Global Array Toolkit}

The Global Array (GA) toolkit provides an efficient and portable "shared-memory" programming interface for distributed-memory computers. Each process in a MIMD parallel program can asynchronously access logical blocks of physically distributed matrices, without need for explicit cooperation by other processes. Unlike other shared-memory environments, the GA model exposes the programmer to the NonUniform Memory Access (NUMA) timing characteristics of parallel computers, and acknowledges that access to remote data is slower than access to local data. From the user perspective, a global array can be used as if it were stored in shared memory, except that explicit library calls are required to access it. Details of actual data distribution and addressing are encapsulated in the global array objects. Matrices are physically distributed blockwise, either regularly or as the Cartesian product of irregular distributions on each axis. The information on the actual data distribution can be obtained and exploited whenever data locality is important.

Each process is assumed to have fast access to some "local" portion of each distributed matrix, and slower access to the remaining "remote" portion. A very efficient, direct access to the local data is supported. Remote data can be accessed through operations like "get," "put," or "accumulate" (floating point sum-reduction) that involve copying of the globally accessible data to/from process-private buffer space. Processes can communicate with each other by creating and accessing GA distributed matrices, and also (if desired) by conventional message passing. Each GA operation can be categorized as a primitive operation, as being constructed from primitive operations, or as providing access to third-party software. Operations also differ in their implied synchronization and atomicity. Elementary operations, with architecture-specific implementation but a portable user interface, that are invoked simultaneously by all processes, include capabilities to:

- create an array, controlling alignment and distribution

- create an array following a provided template (an existing array)
- destroy an array

- synchronize all processes.

A set of primitive operations may be invoked in true MIMD style by any process - with no implied synchronization with other processes and, unless otherwise stated, with no guaranteed atomicity. These primitives include:

- fetch, store, and atomic-accumulate into a rectangular patch of a two-dimensional array

- gather and scatter array elements

- atomic read and increment an array element

- inquire about the location and distribution of the data

- direct access to local elements of an array to support and/or improve performance of application-specific data-parallel operations.

A set of basic linear algebra subroutine (BLAS)like data-parallel operations has been developed on top of the primitive operations (synchronization is included as a user convenience). These include:

- vector operations (e.g., dot-product or scale), optimized by direct access to local data to avoid communication

- matrix operations (e.g., symmetrize, transpose or multiply), optimized by direct access to local data to reduce communication and data copying.

The vector, matrix multiply, copy, and print operations exist in two versions that operate on either entire array(s) or specified sections of array(s). In operations involving multiple arrays, the array sections do not have to be conforming. The only requirements are that the array sections must be of the same type and contain the same number of elements. The data-parallel operations must be invoked simultaneously by all processes. Data consistency is guaranteed only for multiple read, multiple accumulate, or multiple disjoint write operations.

Additional functionality is provided through a variety of third-party libraries made available by 
using the GA primitives to perform the necessary data rearrangements. These include:

- standard and generalized real symmetric eigensolvers

- linear equation solver (interface to SCALAPACK).

The $\mathrm{O}\left(\mathrm{N}^{2}\right)$ cost of data rearrangement is observed to be negligible in comparison to the $\mathrm{O}\left(\mathrm{N}^{3}\right)$ cost of linear-algebra operations. Internally, these libraries may use any form of parallelism appropriate to the host computer system, such as cooperative message passing or shared memory.

The Global Array interface has been designed in the light of emerging standards. In particular, HPF-1 and HPF-2 will certainly provide the basis for future standards definition of distributed arrays in FORTRAN. A long-term goal must be to migrate to full language support, and to eliminate as much as possible the practice of parallel programming through subroutine libraries. The basic functionality described above (create, fetch, store, accumulate, gather, scatter, data-parallel operations) may be expressed as single statements using FORTRAN-90 array notation and the data-distribution directives of HPF. However, HPF currently precludes the use of such operations on shared data in MIMD parallel code. The Global Array tools have been implemented on the Intel parallel computers, the IBM SP1 (under the EUIH message-passing library), the Cray T3D, UNIX workstation networks, multiprocessor UNIX computers using shared memory, and the Kendall Square Research KSR-2 parallel computer.

\section{Global Array Visualizer}

As an aid to tuning the performance of applications using global arrays, a visualization and animation tool has been developed. This tool helps the programmer design efficient task scheduling strategies for MIMD algorithms that operate on the distributed two-dimensional data. The Global Array Visualizer is used to animate access patterns to sections of two-dimensional arrays, through trace data that is gathered in a file during the program execution. Color coding is used to differentiate levels of access contention for particular data blocks. After the animation of events recorded in a tracefile is completed, a composite access contention index is displayed for the entire distributed array, using a different color coding. The tool was applied to our distributed self-consistent field program (see page 4-10). Our first scheduling of the tasks realized poor parallel efficiency, but the reason was not apparent from simple timing data. The performance tool showed that significant contention for data was the problem. This problem was readily addressed by reordering the tasks to spread out references to the GA matrices more uniformly, and by caching (in the application code) to eliminate redundant data references. This eliminated most of the time lost due to contention, but the parallel speedup was still not as good as expected. The dynamic visualization (animation) then showed that some large tasks were being scheduled too near the end of the computation, causing a load-balance problem. This was resolved by incorporating a stratified randomizing scheme that approximately preserved the large-to-small order of tasks, necessary for load-balancing; while still reordering tasks of similar size so as to spread out the GA references and avoid contention. The final scheduling is both load-balanced and almost contention-free. The performance of the main computational kernel of the self-consistent field program was improved approximately fourfold by this tuning.

Distributed Matrices and Linear Algebra (D. Elwood, $^{(a)}$ G. I. Fann, ${ }^{(a)}$ and R. J. Littlefield ${ }^{(a)}$ )

(a) Applied Mathematics, EMSL.

Many electronic structure computations are formulated in terms of dense or nearly dense matrices of size approximately $\mathrm{N}$ by $\mathrm{N}$, where $\mathrm{N}$ is the number of basis functions. Two distinct classes of operations are performed on these matrices:

- random access to small blocks, for the purpose of constructing matrices as a function of many one- and two-electron integrals

- linear algebra operations on the entire matrix, such as eigensolving, Cholesky decomposition, linear system solution, inversion, and matrix-matrix multiplication.

Both types of operations must work on distributed matrices if the resulting application is to be truly scalable. This imposes a stringent limit 
on functionality; if even a single linear algebra operation requires that the entire matrix be present on one processor, then the application cannot be scalable.

The limitation on performance is not so severe, however. In many applications, linear algebra operations on large matrices are only a small fraction of the total number of floating point operations. For example, in an SCF application, it is common for $80 \%$ or more of the arithmetic to be devoted to two-electron integral evaluation, with the remaining $20 \%$ or less being spent on diagonalization and other linear algebra operations. This imbalance allows the use of distributed linear algebra routines that have less than perfect scaling, with little impact on the application's overall time-to-solution and scalability. In addition, as applications evolve to work with progressively larger matrices, it becomes increasingly feasible to reorganize the data so as to optimize the performance of expensive linear algebra operations. This is because data reorganization costs (typified by matrix transpose) require only $\mathrm{O}\left(\mathrm{N}^{2} / \mathrm{P}\right)$ time, while expensive linear algebra operations typically require $\mathrm{O}\left(\mathrm{N}^{3} / \mathrm{P}\right)$.

The development and acquisition of distributed linear algebra routines has been a gradual process, driven by the needs of our applications. Much of our early work used replicated data and conventional sequential linear algebra routines. This approach was adequate with small numbers of processors and relatively slow integral evaluation routines. However, as more processors and faster integral generation technology permitted the use of larger basis sets, linear algebra became a bottleneck for both speed and problem size, and we were forced to convert to fully distributed linear algebra routines.

A particular focus of our distributed linear algebra work has been the development of a scalable, fully parallel eigensolver (PeIGS) whose numerical properties satisfy the needs of the chemistry applications. PeIGS solves dense real symmetric standard $(A x=1 x)$ and generalized $(A x=1 B x)$ eigenproblems. The numerical methods used by PeIGS are multisection for eigenvalues ${ }^{12,13}$ and repeated inverse iteration and orthogonalization for eigenvectors. ${ }^{14}$ Accuracy and orthogonality are similar to LAPACK'S DSPGV and DSPEV. ${ }^{15}$ Unlike other parallel inverse iteration eigensolvers in the current literature, PeIGS guarantees orthogonality of eigenvectors, even for arbitrarily large clusters that span processors.

PeIGS is both fast and scalable - on a single processor it is competitive with LAPACK, and parallel efficiency remains high even for large processor counts. For example, in one of our SCF applications, the standard eigenproblem $\mathrm{Ax}=\mathrm{lx}$ was solved for all eigenpairs of a 2053 by 2053 matrix. This computation required only 110 seconds on 150 processors of an Intel Paragon computer, a time-to-solution estimated as 87 times faster than LAPACK for the same problem on a single processor (the full 2053 by 2053 problem is too large to fit on a single processor, so the 1-processor time is extrapolated from smaller problems and confirmed by counts on larger multiprocessors). Scalability for varying numbers of processors is shown in Figure 4.1(a).

The performance of PeIGS depends to some extent on how eigenvalues cluster within the spectrum. Generally speaking, systems with many eigenvalues close together take longer to solve, due to increased computation and communication costs for reorthogonalization. This is an important issue because actual applications with large matrices often have highly clustered spectra. For example, Figure 4.1(b) shows the spectrum of the 2053 by 2053 matrix referred to above.

Internally, PeIGS uses a conventional messagepassing programming model and columndistributed matrices. However, it is more commonly accessed through an interface provided by the GA toolkit, which supports one-sided data transfers and block-distributed matrices. The necessary data reorganization is handled by the interface. As noted above, such reorganization is very fast compared to the $\mathrm{O}\left(\mathrm{N}^{3} / \mathrm{P}\right)$ times required for linear algebra operations.

PeIGS runs on a variety of parallel computer systems and can be ported easily to any machine that supports message passing. The current version of PeIGS utilizes either the TCGMSG portable communication package or Intel NX intrinsics; an MPI port is planned. PeIGS is being used at EMSL as a production-grade library package in the construction of NWChem modules, including parallel SCF, MCSCF, DFT, and MP2 methods. Due to the speed and scalability of PeIGS, the time for eigensolving and associated 


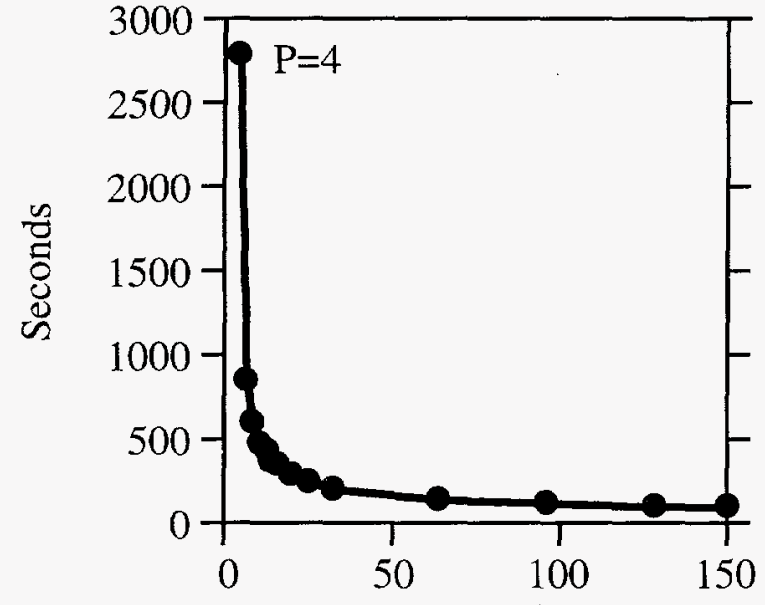

(a) Processor Count, $\mathrm{P}$

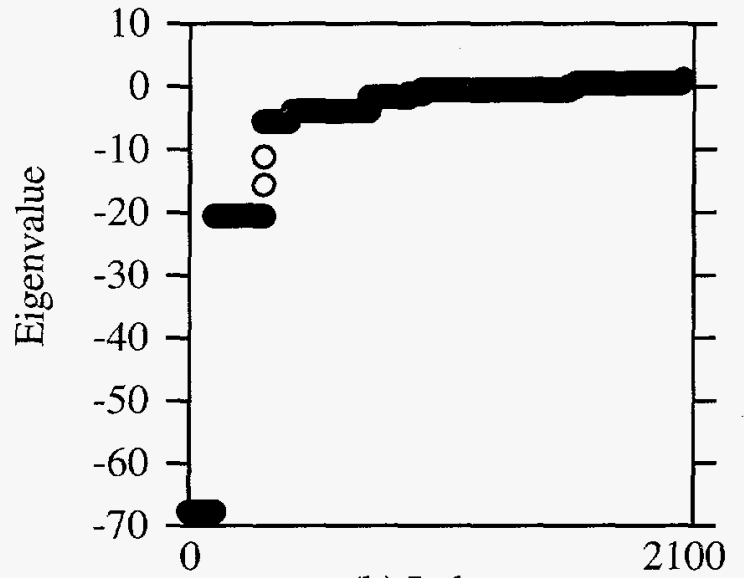

(b) Index

Figure 4.1. Scalability (a) for varying numbers of processors for the determination of all eigenpairs of a 2053 by 2053 matrix whose spectrum is displayed in (b).

linear algebra operations has been reduced to a small fraction of the total time-to-solution for these applications.

Run Time Database (R. J. Harrison and R. A. Kendall)

The run time database (RTDB) is the parameter and information repository for all application modules comprising NWChem. This is similar in spirit to the GAMESS ${ }^{16-19}$ dump file or GAUSSIAN ${ }^{20}$ checkpoint file. An input parsing module(s) stores the input, and each application module obtains the appropriate parameters for execution and communicates to other modules via the RTDB. The RTDB library is accessible from both FORTRAN and $\mathrm{C}$, is based on simple UNIX database tools, and is widely portable. The storage is based on a string-to-data (of a single type) mapping, with complex data structures stored as separate data blocks of the appropriate type. Contextual levels are also built into this tool; for example, the gradient convergence threshold or the print level are parameters that may be applicable to various modules, and with a different value for each method/module. The appropriate context can be set to arbitrate among the possible values.

\section{Parallel Computational Chemistry Software}

We present in this section an outline of the development, implementation, and performance of a variety of the modules that make up NWChem. Following an outline of the common components of the chemistry applications, we consider the attributes of a highly efficient and scalable algorithm to perform quadratically convergent, SCF (QCSCF) Hartree-Fock calculations on molecular systems. We then provide a brief account of the development of corresponding capabilities using the so-called "resolution of the identity" (RI) integral approximation. ${ }^{21}$ The description of the parallel periodic Hartree-Fock capabilities is addressed later in the section entitled Collaboration in Molecular Science Software: Daresbury Laboratory - Pacific Northwest National Laboratory. Algorithmic developments involved in performing the so-called integral transformation, a necessary step in moving from the SCF to more quantitative methods, are then considered, followed by some initial thoughts on performing "direct" MCSCF calculations. Two implementations of MP2, designed to be optimum in the treatment of both spatially extended and compact molecules: "Mixed basis" MP2 and RI-MP2 follow. Finally we turn to consider the parallel implementation of DFT, an increasingly popular and truly costeffective alternative to more conventional correlated $a b$ initio methods.

We discuss the implementation of molecular dynamics (MD) capabilities and the consideration of the algorithmic issues involved in treating longrange interactions as well as our activities in developing techniques to combine quantum mechanics and molecular mechanics in the 
section devoted to the Daresbury Laboratory Pacific Northwest National Laboratory collaboration.

The performance figures presented throughout this section were derived from runs on both the Intel Touchstone Delta and Kendall Square Research KSR-2 supercomputers.

\section{Common Software Components (R. A. Kendall)}

The software tools outlined previously are examples of libraries with a standard application programmer interface (API). Designing a standard API for each functionality that is required by many of our codes promotes code reuse, decreases the effort needed to develop new applications, and also hides complexity - which further decreases development and maintenance costs. For example, when accessing an element of a global array, the application module does not require specific knowledge of where the data resides or whether the data is retrieved via a message-passing call or some shared-memory mechanism. If it is subsequently necessary to change the implementation details of a GA, the application is insulated from such changes. Many chemistry-specific components can be similarly defined; and with proper design and implementation, we may realize similar benefits. Figure 4.2 illustrates the architecture of our applications in a two-dimensional block diagram, although the actual interplay is multidimensional.

This design may be applied at all levels within the code. For instance, our ab initio molecular dynamics module invokes the self-consistent field and self-consistent field gradient modules as simple components through a standard API. To solve the SCF equations requires neither access to the complete details of the basis set of the chemical system, nor the exact position of the atomic centers. The SCF module may need to know the total numbers of centers and contracted functions, but only the integral evaluation package will require actual coefficients and exponents of the primitive Gaussian basis. To illustrate this further, we outline the basis set and geometry objects and the integral API used in the NWChem software.

The geometry object specifies the physical makeup of the chemical system by defining

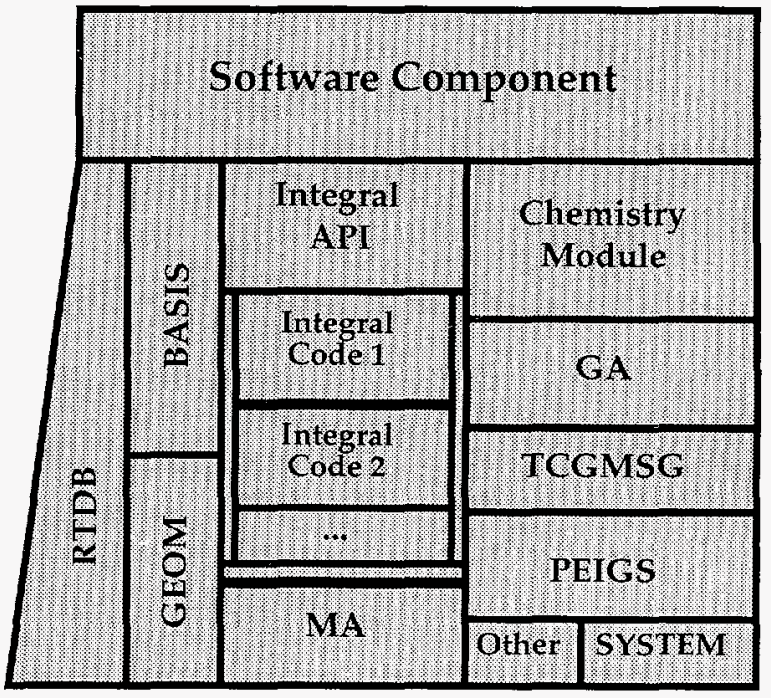

Figure 4.2. The interplay of software tools, common software components, and specific module software.

atomic centers, their respective positions in space and nuclear charges, as well as an associated name for each center. It also includes a possible applied electric field, the symmetry (point group or periodic) and other characteristics of the system. The basis set object handles the details of the Gaussian basis set (i.e., the approximate atomic orbitals), and stores only the unique information for each center. The combination of a basis set object and a geometry object provides all non-state-specific information required by an $a b$ initio calculation. These are not true "objectoriented" objects, since we do not support inheritance; but they are well-defined APIs that enforce strict data hiding. Such a design allows the utilization of multiple basis sets and geometries throughout an application module. By allowing simple access to different "instances" of these objects this design has reduced the burden of implementing such techniques as the density functional theory, RI-self-consistent field, RI-MøllerPlesset perturbation, etc., which require multiple basis sets.

All of the computational chemistry applications described below require, at some level, one- and two-electron integrals and integral derivatives over the Gaussian basis set(s) of the system. The integral API is a layer between the base integral code and the application module, and allows the application programmer to essentially ignore the 
details of how the integrals are computed. This facilitates the incorporation of different integral technology into the applications that use the code; currently, the API requests integrals of various types based on computing shell blocks of integrals, with two different base integral codes used for production. The in-house developed code is a straightforward, open-ended implementation of the McMurchie-Davidson 22,23 algorithm.

Self-Consistent Field Hartree-Fock (H. A. Früchtl, ${ }^{(a)}$ R. A. Kendall, R. J. Harrison, and A. T. Wong ${ }^{(a)}$ )

(a) AWU Postdoctoral Research Associate

The essential core functionality in an electronic structure program suite is the direct SCF module. It is increasingly evident that the application of direct SCF to large molecules is best performed on MPPs, due to the enormous computational requirements. However, targeting systems in excess of 1,000 atoms and 10,000 basis functions requires a re-examination of the conventional algorithm and assumed memory capacities. The majority of previous parallel implementations used a replicated-data approach, ${ }^{4}$ which is limited in scope because the size of these arrays will eventually exhaust the available single-processor memory. Our implementation of parallel direct SCF distributes these arrays across the aggregate memory, using the GA tools. This ensures that the size of systems that can be treated scales with the size of the MPP, and is not constrained by single-processor memory capacities.

The computationally dominant step, the construction of the Fock matrix, is readily parallelized because the integrals can be computed concurrently. With distributed matrices, a stripmining approach is used, where the integral contributions to small blocks of the Fock matrix are computed locally and accumulated asynchronously into the distributed matrix. By choosing blocking over atoms, the falloff in interaction between distant atoms can be exploited, while simultaneously satisfying local memory constraints. A simple performance model indicates that the ratio of computation to communication remains high, as shown in Figure 4.3, which demonstrates the scalability of this algorithm for a medium-sized system.
The conventional SCF solution scheme is based on repeated diagonalization of the Fock matrix. In the quadratically convergent SCF approach, 24 the SCF equations are recast as a non-linear minimization, which bypasses the diagonalization step. This scheme consists of only data-parallel operations and matrix multiplications, which guarantees high efficiency on parallel machines.

Perhaps more significantly, QCSCF is amenable to several performance enhancements that are not possible in conventional approaches. For instance, orbital-Hessian vector products may be computed approximately (e.g., using the resolution of the identity approximation discussed below), which significantly reduces the computation expense with no affect on the final accuracy. Figure 4.3 shows the speedup for the distributed Fock matrix construction and the complete QCSCF algorithm for a modest 461 basis function calculation of disilasesquioxane, on both the Intel Touchstone Delta (Figure 4.3[a]) and the KSR-2 (Figure 4.3[b]). When the number of processors approaches $N^{2} / 2$, where $N$ is the number of atomic orbitals in the system, the speedup for Fock matrix construction is degraded, due to load-balancing requirements. The overall QCSCF speedup for small molecular calculations is also affected by the finer distribution of the global data structures as the number of nodes increases. In the disilasesquioxane case, neither effect is evident; the scalability of the QCSCF algorithm using distributed data has been demonstrated up to 512 nodes with systems of $N \sim 3500$.

\section{Resolution of the Identity - Self-Consistent Field}

One way to reduce the computational expense associated with the calculation of the four-center two-electron repulsion integrals is to approximate these integrals as linear combinations of three-center integrals, using the RI integral approximation. $^{21}$ The idea is that products of Gaussian functions of the atomic orbital (AO) basis are approximated by linear combinations of Gaussians in a separate "fitting" basis. If the fitting or expansion basis were chosen to be the full Gaussian Product Theorem (GPT) result of all possible combinations of the AO basis functions, the RI result would be exact. ${ }^{25}$ Vahtras et al. ${ }^{26}$ have investigated several ways of expanding the 

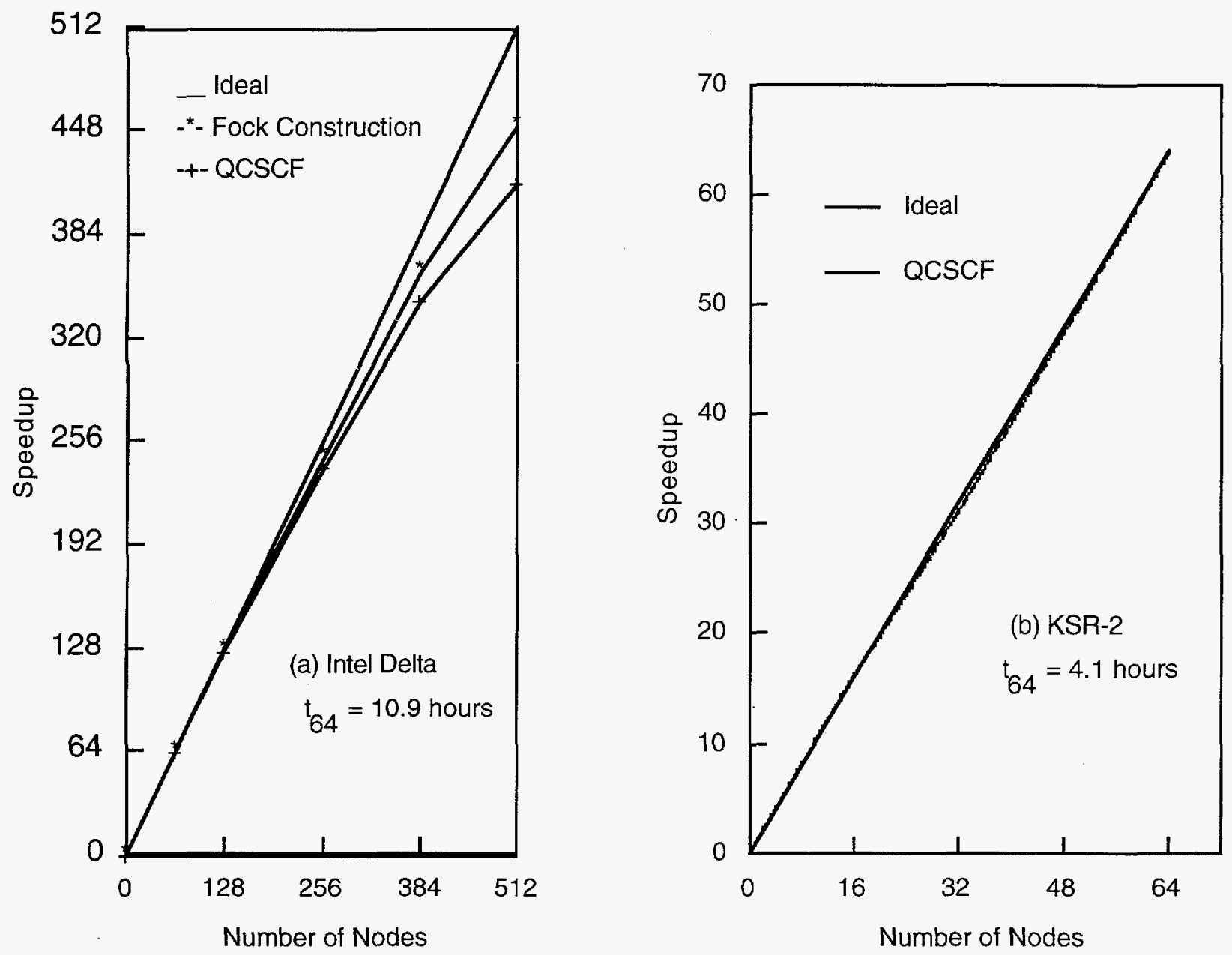

Figure 4.3. Quadratically convergent SCF (QCSCF) performance (a) on the Intel Touchstone Delta and (b) on the KSR-2.

four-center integrals in terms of three-center integrals, with the so-called V-approximation best suited to reproduce total and dissociation energies;

$$
(\mathrm{ij} \mid \mathrm{kl})=\sum_{\mathrm{tu}}(\mathrm{ij} \mid \mathrm{t})\left[\mathrm{V}^{-1}\right]_{\mathrm{tu}}(\mathrm{u} \mid \mathrm{kl})
$$

where

$$
\mathrm{V}_{\mathrm{tu}}=(\mathrm{t} \mid \mathrm{u})
$$

The expansion basis, which approximates products (or the potential arising from products) of basis functions, has to contain more functions and higher angular momenta than the $\mathrm{AO}$ basis. In our calculations, we used atom-centered, even- tempered, uncontracted basis sets with maximum angular momenta two higher than the maximum angular momenta found in the $\mathrm{AO}$ basis. The three-center electron repulsion integrals are calculated only once and are transformed with $\mathrm{V}^{-1 / 2}$, which simplifies the subsequent calculation of the four-center contributions to

$$
\begin{aligned}
(\mathrm{ij} \mid \mathrm{kl}) & =\sum_{\text {stu }}(\mathrm{ij} \mid \mathrm{s})\left[\mathrm{V}^{-1 / 2}\right]_{\mathrm{St}}\left[\mathrm{V}^{-1 / 2}\right]_{\mathrm{tu}}(\mathrm{u} \mid \mathrm{kl}) \\
& =\sum_{\overline{\mathrm{t}}}(\mathrm{ij} \mid \mathrm{t})(\overline{\mathrm{t}} \mid \mathrm{kl})
\end{aligned}
$$

Only these transformed integrals are stored as a GA, effectively utilizing the (possibly distributed) memory of the parallel computer. 
The calculation of the Coulomb contribution to the Fock matrix

$$
J_{\mathrm{ij}}=\sum_{\overline{\mathrm{t} k l}}(\mathrm{ij} \mid \overline{\mathrm{t}})(\overline{\mathrm{t}} \mid \mathrm{kl}) \mathrm{D}_{\mathrm{kl}},
$$

is most efficiently formulated as two sparse matrix-vector products

$$
\begin{aligned}
& W_{\mathrm{t}}^{-}=\sum_{\mathrm{kl}}(\overline{\mathrm{t}} \mid \mathrm{kl}) D_{\mathrm{kl}} \\
& \mathrm{J}_{\mathrm{ij}}=\sum_{\mathrm{t}}(\mathrm{ij} \mid \tilde{\mathrm{t}}) \mathrm{W}_{\mathrm{t}}^{-}
\end{aligned}
$$

which is a computational effort of order $\mathrm{O}\left(\mathrm{N}^{3}\right)$, assuming that the number of expansion functions is proportional to the number of AOs.

The exchange component of the Fock matrix elements

$$
K_{\mathrm{ij}}=\sum_{\mathrm{t} \mathrm{kl}}(\mathrm{ik} \mid \overline{\mathrm{t}})(\overline{\mathrm{t}} \mathrm{jl} \mathrm{l}) \mathrm{D}_{\mathrm{kl}} \text {, }
$$

is not so easily partitioned, and has to be computed as two matrix multiplications

$$
\begin{aligned}
\mathrm{T}_{\mathrm{jk}}^{\overline{\mathrm{t}}} & =\sum_{1}(\overline{\mathrm{t}} \mid \mathrm{j} 1) \mathrm{D}_{\mathrm{kl}}, \\
\mathrm{W}_{\mathrm{ij}}^{\overline{\mathrm{t}}} & =\sum_{\mathrm{k}}(\mathrm{ik} \mid \overline{\mathrm{t}}) \mathrm{T}_{\mathrm{jk}}^{\overline{\mathrm{t}}} \text {, and } \\
\mathrm{K}_{\mathrm{ij}} & =\sum_{\tilde{\mathrm{t}}} \mathrm{W}_{\mathrm{ij}}^{\overline{\mathrm{t}}} .
\end{aligned}
$$

Under the assumptions listed above, this part of the calculations scales as $\mathrm{O}\left(\mathrm{N}^{4}\right)$, but as it consists only of linear algebra and does not include the calculation of integrals, it is executed much faster than the corresponding step in direct SCF.

Figure 4.4 shows the speedup curve on our KSR-2 computer for a calculation on propane using a ccpVTZ basis set. The expansion basis was eventempered and uncontracted, with 5s4p3d2f1g for hydrogen and $6 \mathrm{~s} 5 \mathrm{p} 4 \mathrm{~d} 3 \mathrm{f} 2 \mathrm{~g} 1 \mathrm{~h}$ for carbon. The method scales well with an efficiency of more than $80 \%$ up to 64 nodes, with a total 64 -node run time of 396 seconds as compared to 4200 seconds for direct SCF with four-center integrals. The total energy calculated with this RI method is within 0.0005 Hartree of the full SCF energy using the same $\mathrm{AO}$ basis.

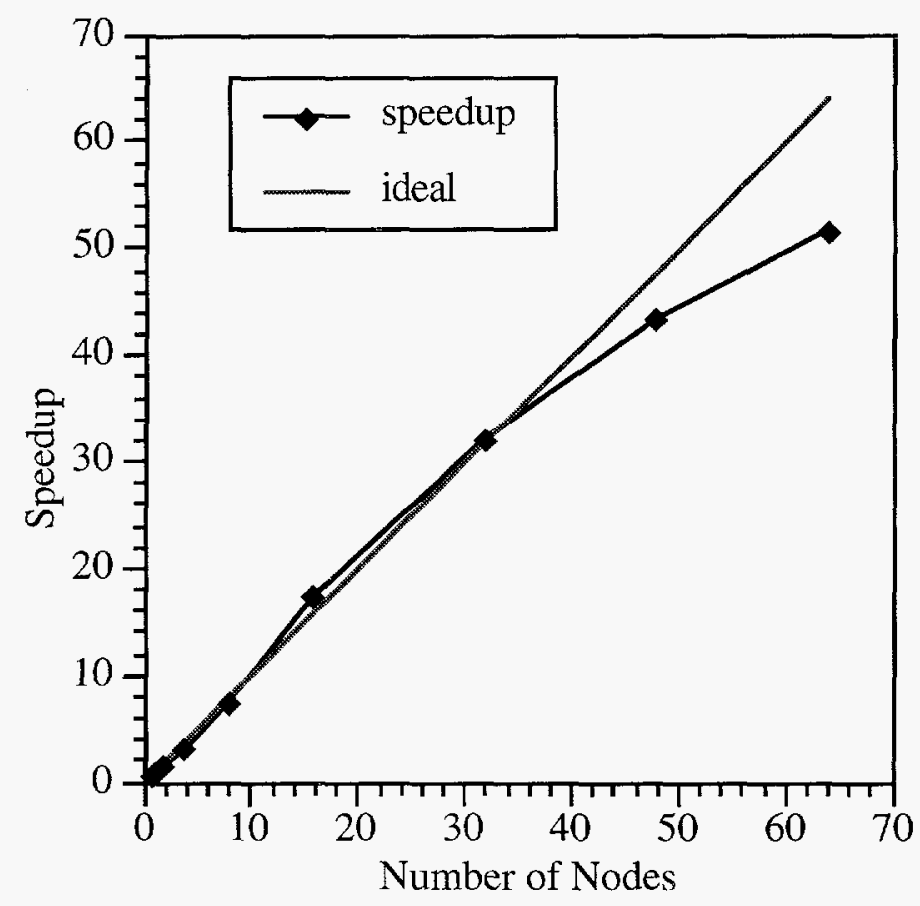

Figure 4.4. RI-SCF speedup curve on the KSR-2 for a calculation on propane using a cc-pVTZ basis set. 
These results show that the RI-SCF method is a fast and efficient way to calculate approximate SCF energies, especially for compact systems with extensive basis sets. Approaches for larger systems ( $>\approx 400$ basis functions), where in-core storage of the transformed integrals is no longer practicable, are currently under investigation. Also, a more systematic approach must be found to the choice of expansion basis sets, especially for spatially extended systems with diffuse functions. Presently in such cases, atom-centered functions give only a poor approximation to the product functions.

Direct Four-Index Transformation (R. J. Harrison, A. P. Rendell, ${ }^{(a)}$ and A. T. Wong ${ }^{(b)}$ )

(a) EPSRC Daresbury Laboratory, Daresbury, UK

(b) AWU Postdoctoral Research Associate

The four-index transformation of AO integrals to the molecular orbital $(\mathrm{MO})$ representation is the intermediate step in moving from SCF calculations to more sophisticated correlated methods. This procedure would be difficult to implement efficiently on parallel architectures using traditional disk-based approaches. However, by computing the $\mathrm{AO}$ integrals as required, analogous to direct SCF, the four-index transformation can be very efficiently performed. Given sufficient parallelism and low communication overhead, the computation of integrals combined with the transformation can readily extract almost peak computational rates from each processor (for example, 3.5 Gflops were recently obtained from 128 nodes of the Intel Touchstone Delta). As in the SCF method, the judicious use of memory is the overriding issue in the design of an algorithm capable of treating large systems. Our current four-index transformation routine has $\mathrm{O}\left(\mathrm{N}^{2}\right)$ local memory requirements, a significant improvement over available programs which typically assume $\mathrm{O}\left(\mathrm{N}^{3}\right)$ memory requirements. Figure 4.5 plots the speedup obtained for a prototype version which exhibits acceptable scalability, although the performance model for our latest version indicates that a much higher level of parallelism can be achieved by removing synchronization points.

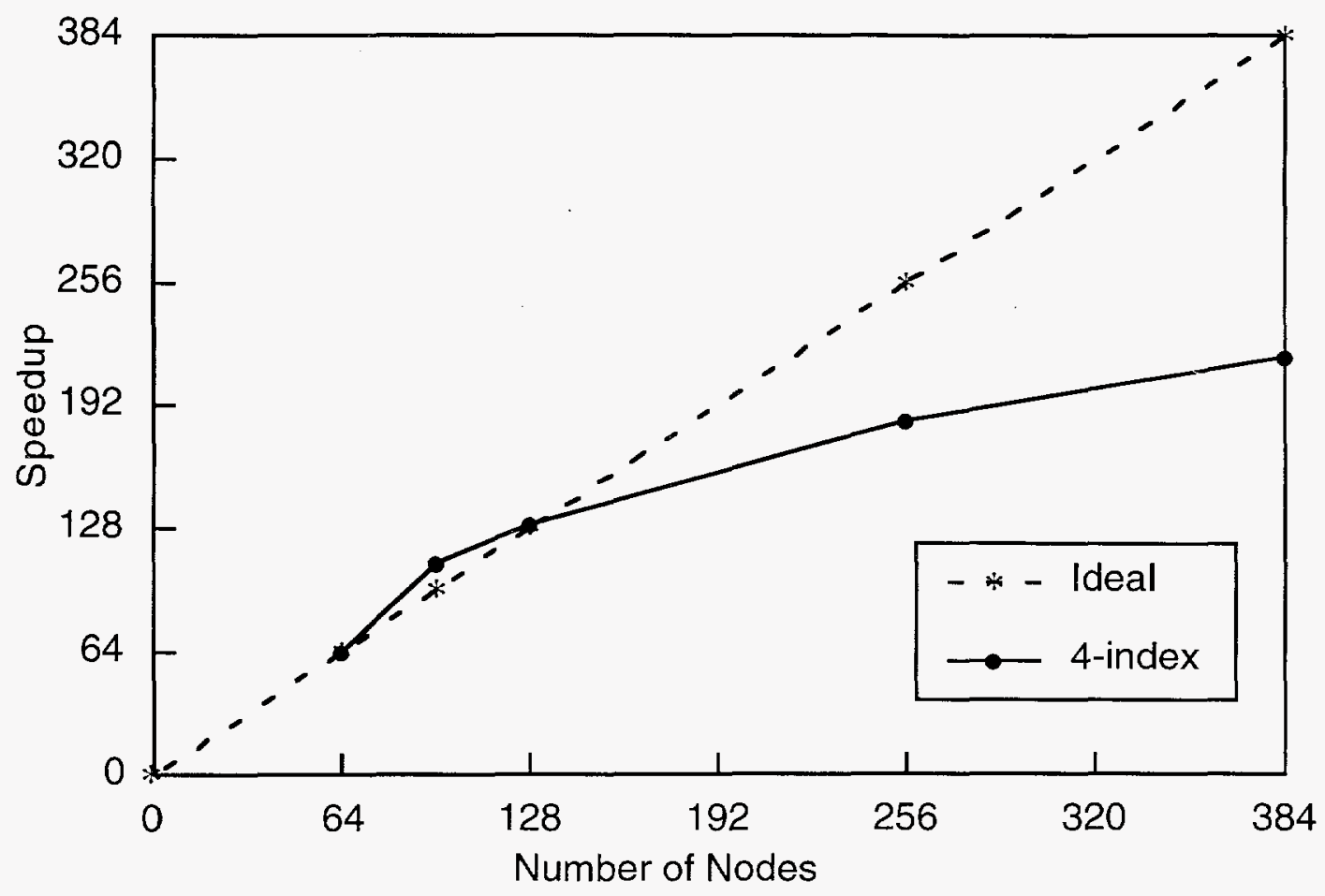

Figure 4.5. Speedup obtained for a 4-index transformation on 18-crown-6 ether (114 basis functions) using the Intel Touchstone Delta. 
Direct Multiconfiguration Self-Consistent Field (R. J. Harrison and A. T. Wong ${ }^{(a)}$ )

(a) AWU Postdoctoral Research Associate

MCSCF, a generalization of the SCF method, is an indispensable tool in studies of intermediate and reactive species. The traditional MCSCF procedure requires the transformation of integrals and solution of the secular problem, which scales as $n_{a}$ ! where $n_{a}$ is the number of active orbitals. As a consequence of the high computational demand of these two components, MCSCF is currently limited to relatively small systems. The number of transformed integrals computed and stored externally can be significantly reduced in a direct ${ }^{\dagger}$ approach equivalent to QCSCF. 27 Although such a technique is not economic for sequential computers, on MPPs the recomputation of integrals at each optimization step is an acceptable cost, compared to external storage of these integrals. There is ongoing work within the HPCC group to produce a direct MCSCF program, which should significantly expand the capability of this method.

The ability to treat an MCSCF wave function with a moderately-sized active space is problematic because of the factorial scaling in the secular component. Even taking into account the large aggregate memory capacity envisioned in future MPPs, the size of the active space is expected to increase only twofold. For cases which demand large active spaces, such alternatives as the restricted active space (RAS) method, ${ }^{28}$ will be necessary.

Møller-Plesset Perturbation Theory (D. E. Bernholdt ${ }^{(a)}$ and R. J. Harrison)

(a) AWU Postdoctoral Research Associate

The Hartree-Fock SCF method can be improved upon by methods that incorporate the effects of dynamic electron correlation. The use of correlated methods is often necessary in order to obtain predictive "chemical" accuracy. The simplest such method is MP2, which has proven use-

+ This term is also used to describe MCSCF solution schemes that do not store the Hessian explicitly, a technique that is also used in our current implementation. ful in a wide range of problems for which the Hartree-Fock method is inadequate.

The MP2 method involves $\mathrm{O}\left(\mathrm{N}^{5}\right)$ computational work and $\mathrm{O}\left(\mathrm{N}^{4}\right)$ data, as compared with $\mathrm{O}\left(\mathrm{N}^{4}\right)$ and $\mathrm{O}\left(\mathrm{N}^{2}\right)$, respectively, for the SCF. While MP2 presents many problems characteristic of more sophisticated correlated methods (most notably the large volume of data to be dealt with), it is also worth noting that (like the SCF method) most other correlated methods involve a factor of $\mathrm{N}^{2}$ more computation than data. Consequently, MP2 is one of the tougher problems for parallel performance in computational chemistry.

As computational and algorithmic advances have made calculations on progressively larger chemical systems possible, it has become clear that despite the formal $\mathrm{O}\left(\mathrm{N}^{5}\right)$ cost of the method, characteristics of many chemical systems will allow for lower-cost calculations if "non-traditional" algorithms are employed. Alternative methods involve new ways of representing the interactions within the system exactly, using new mathematical formulations, controllable approximations, or a combination of both. In our work on large-scale correlated calculations on MPPs, we have chosen to pursue two of these alternative approaches that are particularly well suited to different problem domains: the "mixed-basis" approach and the RI-MP2 method.

\section{Mixed Basis Møller-Plesset Perturbation Theory}

The traditional MP2 algorithm uses MOs as the basis for computing interactions in the system. Unfortunately, these MOs are usually distributed across many atoms in the molecule. Consequently the interactions, which depend on distance, are rarely small enough to neglect. However, by recasting the problem so that a more spatially localized basis can be used, it should be possible to introduce significant sparsity into the interactions. The new representation involves localizing the occupied MOs and representing the unoccupied MOs, which are not easily localized, in the AO basis. This is the origin of the term "mixed basis". which we use to describe this method. The mixed basis formulation should be well suited to spatially extended chemical systems with moderately sized basis sets. 
We have a prototype fully distributed parallel mixed-basis MP2 code that, for some relatively small systems, shows good scalability on the Intel Touchstone Delta. Figure 4.6 illustrates the speedup on a calculation on butane, $\mathrm{C}_{4} \mathrm{H}_{10}$, with an augmented pVDZ basis. The two dominant computational steps are the construction of the exchange operators and the determination of the excitation amplitudes. Exceptional speedup is shown in the first step, while degradation occurs in the second, due to the smallness of the system and the relatively high ratio of communicationto-computation time. This code will be used as a testbed to determine the most efficient way to take advantage of sparsity. Without using very high performance parallel computers, it has not been possible to routinely perform calculations large enough to exhibit sufficient sparsity to be interesting in this context. We believe that once sparsity is incorporated in the parallel code, we will be able to treat systems on the order of 1000 3000 basis functions, or 100-300 atoms - nearly an order of magnitude larger than the largest reported MP2 calculations to date. The much larger amount of data involved in an MP2 calculation will limit the size of calculation unless scalable I/O facilities are available to make disk storage of some of the data practical.

\section{Resolution of the Identity - Moller-Plesset Perturbation Theory}

Another type of system of particular interest is one which is relatively compact in spatial terms but for which large basis sets are used. In this case, the physical proximity of the atoms and characteristics common to large basis sets results in very few of the interactions being negligibly small.

For problems of this type, we have implemented a parallel RI-MP2 code which uses the RI integral approximation. ${ }^{21}$ As mentioned in the previous section, if the associated fitting basis were chosen to be the full GPT result of all possible combinations of the $\mathrm{AO}$ basis functions, the $\mathrm{RI}$ result

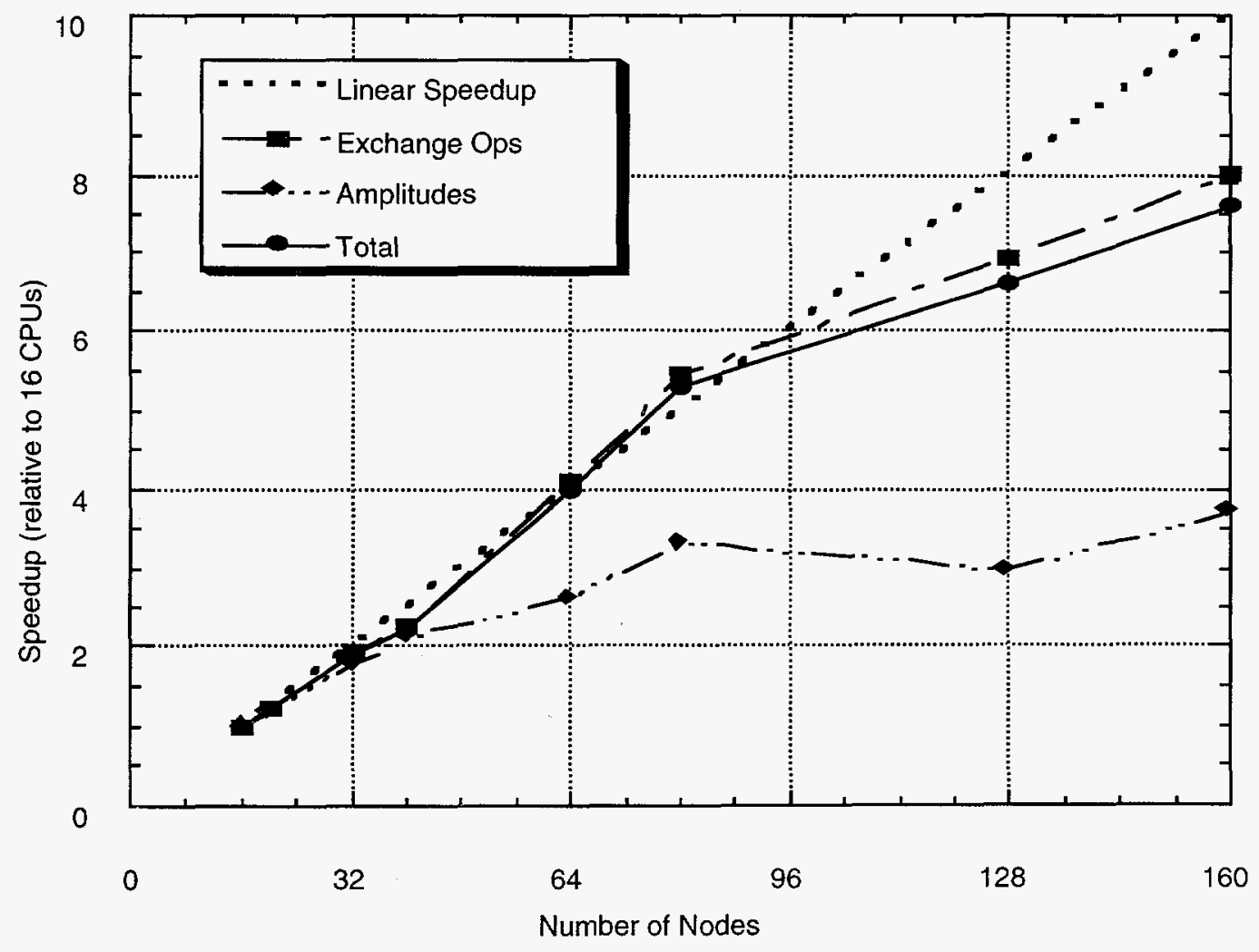

Figure 4.6. Mixed-basis MP2 speedup obtained on the Intel Touchstone Delta for a calculation on $\mathrm{C}_{4} \mathrm{H}_{10}$ with an augmented $p V D Z$ basis. 
would be exact. ${ }^{25}$ However, experience has shown that the GPT basis is generally very nearly linearly dependent, so it should be possible to reduce the fitting basis and still retain most of the accuracy of the calculation. In general, the larger the $\mathrm{AO}$ basis set, the more near-redundancy in the GPT basis which is why we feel the RI-MP2 method is particularly suited to compact systems with relatively large basis sets.

Using the RI approximation changes the amount of data that must be dealt with in most of the MP2 procedure from $\mathrm{O}\left(\mathrm{N}^{4}\right)$ to $\mathrm{O}\left(\mathrm{N}^{3}\right)$ - significant because this is the amount of data that is stored on disk if there is not enough memory to do the whole calculation in core. The computational dependence of the RI-MP2 remains $\mathrm{O}\left(\mathrm{N}^{5}\right)$, but it may be formulated as a large distributed matrix multiplication, which performs well on MPPs. The RI-MP2 procedure can be expressed very straightforwardly in terms of the GA toolkit and is implemented in such a way as to require only $\mathrm{O}\left(\mathrm{N}^{2}\right)$ memory, provided that $\mathrm{O}\left(\mathrm{N}^{3}\right)$ disk space is available, making it possible to perform RI-MP2 calculations on most systems for which an SCF is feasible.

In Figure 4.7 we show the speedup obtained on the KSR-2 for calculations on 2,2'- di(trifluoromethyl)biphenyl, using 204 AO basis functions and 966 fitting functions. The primary components of the overall timing are the integral transformation and the energy evaluation. The energy evaluation, which is dominated by a large distributed matrix multiplication, displays excellent speedup over a range from 5 to 70 processors. The transformation also scales well, but suffers from a degree of load imbalance in one step which reduces the performance slightly compared to the energy evaluation. Although for this case the transformation and energy components require comparable times, they have different computational complexities; for larger problems, the energy evaluation component will dominate. Although these runs were disk-based, I/O time on the KSR is a negligible part of the run time on this problem because of the small volume of data (compared to an exact MP2 calculation) and the buffering of the KSR's I/O subsystem and operating system. With this implementation in hand, we feel that the most important area for further work on the RI-MP2 method is not in parallelization, but rather in the development of fitting basis sets. Because this approach is very recent, ${ }^{21}$ there is little detailed information available as yet on how to construct fitting basis sets to achieve given levels of accuracy. As part of this development effort, the parallel RI-MP2

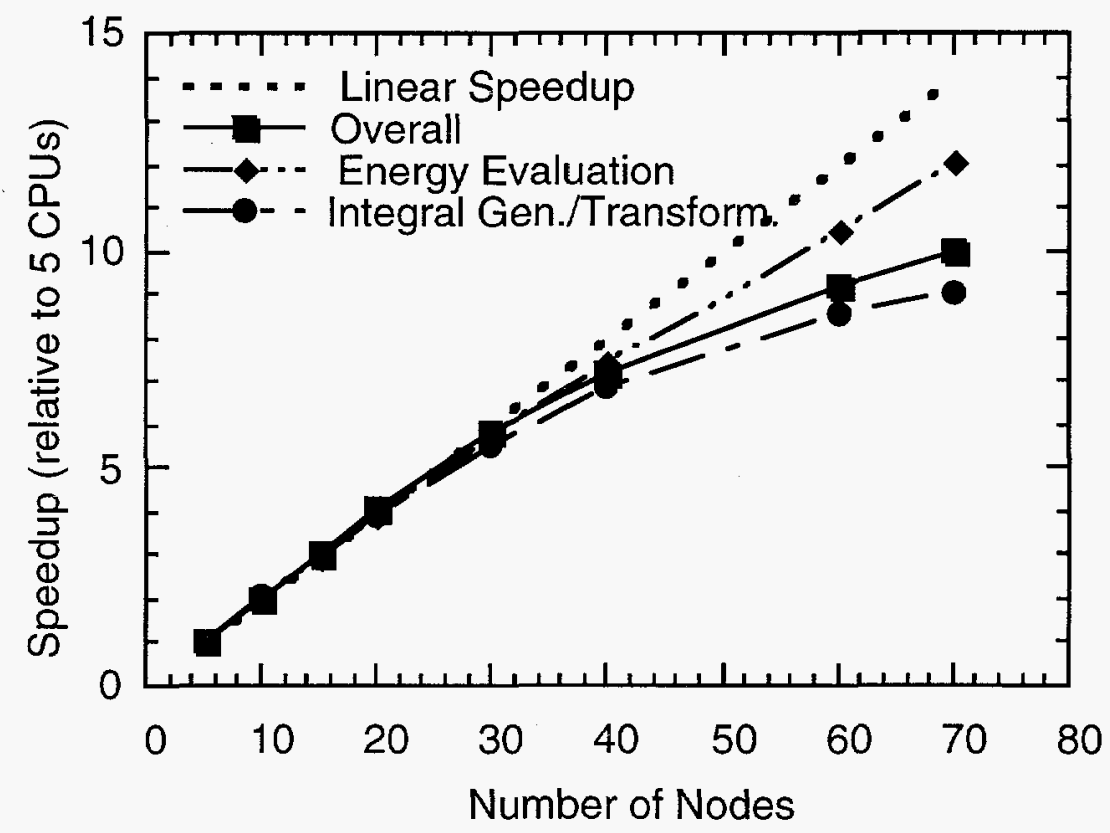

Figure 4.7. RI - MP2 speedup obtained on the KSR-2 for calculations on 2,2'-di(trifluoromethyl)biphenyl, using $204 \mathrm{AO}$ basis functions and 966 fitting functions. 
will allow a much broader range of systems to be examined rapidly.

Density Functional Theory (J. A. Anchell, ${ }^{(a)}$ E. Aprà, $^{(b)}$ A. C. Hess, ${ }^{(c)}$ J. E. Jaffe, ${ }^{(d)}$ J. A. Nichols, and H. L. Taylor ${ }^{(b)}$ )
(a) KSR Inc.
(b) AWU Postdoctoral Research Associate
(c) Solid State Theory Group
(d) AWU Visiting Scientist, Solid State Theory Group

The DFT software makes extensive use of the tools described above, including MA, TCGMSG, and GA. All data objects larger than $O(N)$ are stored in GAs. The code we have developed is a MPP implementation of the Hohenberg-KohnSham formalism ${ }^{29,30}$ of DFT. This method yields results similar to those from correlated $a b$ initio methods, at substantially reduced cost. It assumes a charge density and approximations are made for the Hamiltonian (the exchange correlation functional); in contrast with traditional $a b$ initio molecular orbital methods (see above) that assumes an exact Hamiltonian and chooses approximations to the wavefunction. ${ }^{31}$ The Gaussian basis DFT method in NWChem breaks the Hamiltonian down into the same basic oneelectron and two-electron components as traditional methods, with the two-electron component further reduced to a Coulomb term and an exchange-correlation term. The treatment of the former can be accomplished in a fashion identical to that used in traditional SCF methods, from a fitted expression similar to that found in RI-SCF, or from the commonly used Dunlap fit. ${ }^{32,33} \mathrm{DFT}$ is really distinguished from other traditional methods, however, by the treatment of the exchange-correlation term. This term is typically integrated numerically on a grid, or fit to a Gaussian basis and subsequently integrated analytically.

In our prototype DFT code, the electron density and the exchange-correlation functional can be expanded in terms of auxiliary Gaussian basis sets. We have incorporated the exchangecorrelation functionals (Dirac's exchange functional $^{34}$ and the Vosko, Wilk, Nusair correlation functional ${ }^{35}$ ) required to obtain the local density approximation (LDA) and the local spin density approximation (LSD) for open shell systems. In addition, we have implemented twogradient corrected functionals for the exchangecorrelation contributions: the Becke exchange functional $^{36}$ and the Lee, Yang, and Parr correlation functional. ${ }^{37}$ All of these functionals are fully implemented for closed and open shell systems. Several techniques have been incorporated to improve convergence in the iterative SCF procedure: damping, level shifting, and direct inversion in the iterative subspace (DIIS). ${ }^{38-40}$ These have, of course, all been implemented so as to exploit MPP technology with maximum efficiencies.

The time-consuming portions of NWChem DFT calculations have already shown significant speedup on the KSR-2 (in calculations on various zeolite fragments with system sizes ranging up to $1700 \mathrm{AOs}$ and 4000 fitting functions). The four time-consuming steps are: fit of the charge density, calculation of the Coulomb potential, evaluation of the exchange-correlation potential, and diagonalization of the Fock matrix. Overall speedup of better than $90 \%$ is seen, using up to 70 processors to build and diagonalize the Fock matrix. Figure 4.8 shows the speedup obtained on up to 70 nodes of the KSR-2 for $\mathrm{Si}_{26} \mathrm{O}_{37} \mathrm{H}_{30}$ with a DZP basis set.

The scaling of the components of the DFT code (wall clock time versus number of atomic orbitals) was examined for various sized zeolite fragments, using 48 nodes of the KSR-2. In a fit to the function $t=\mathrm{An}^{\mathrm{B}}$ for different components of the calculation, the value $B$ obtained was 2.47, 2.50, 2.11, 2.15, 2.32, 2.33; for $C D$ fit, $V_{C o u l}, V_{X C}$, Diag, DIIS, and Fock+Diag, respectively. The scaling for the overall calculation was given by $\mathrm{B}=2.3$.

The Intel machines (unlike the KSR-2 which has an ALLCACHE "shared memory" architecture) are truly massively parallel distributed memory platforms. In porting to the Touchstone Delta, we encountered a few algorithmic flaws (with respect to data locality) which had to be corrected in order to obtain similar large speedups. 


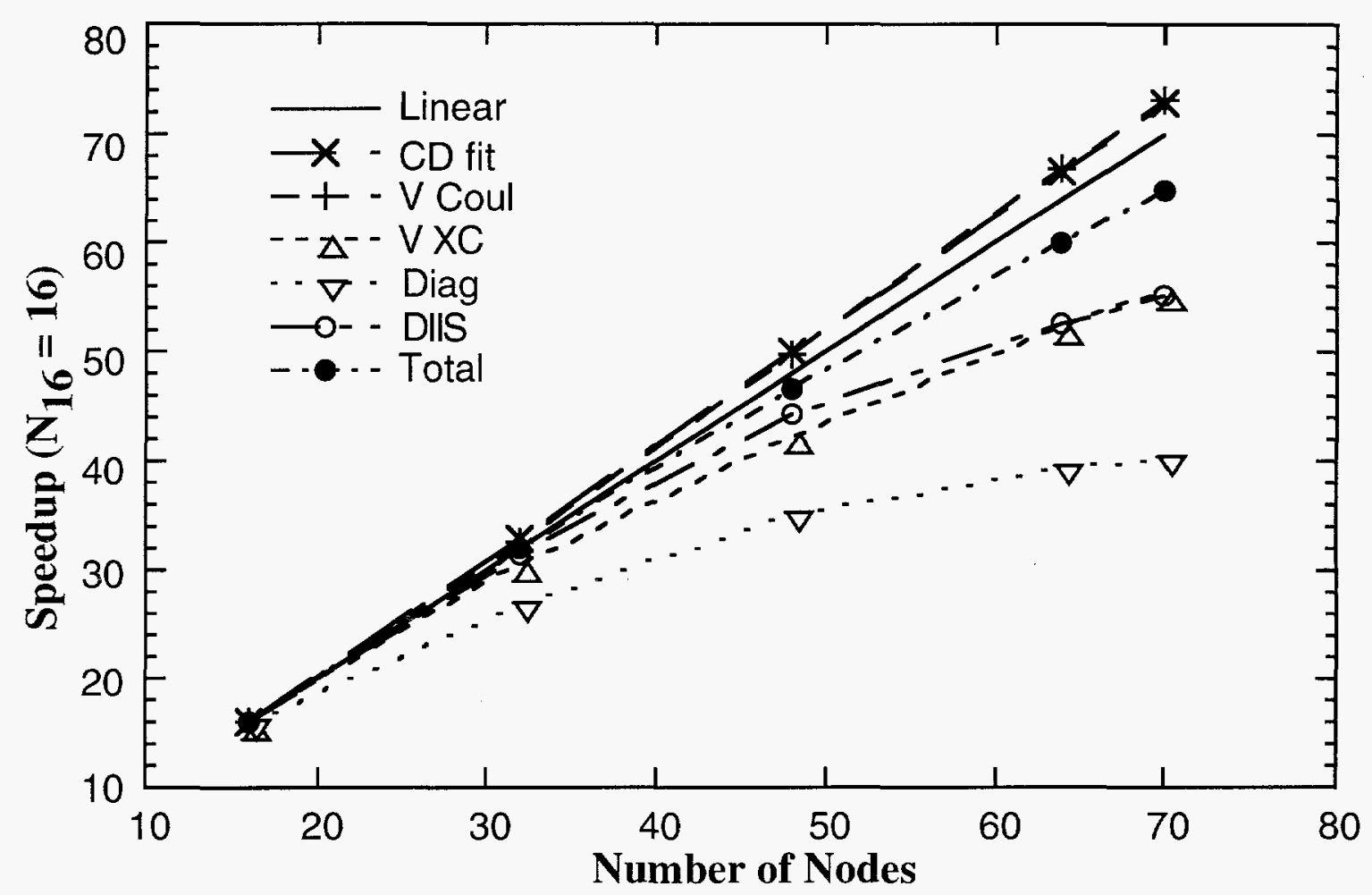

Figure 4.8. Speedups obtained for construction of Fock matrix components and subsequent diagonalization in DFT calculations of the zeolite fragment $\mathrm{Si}_{26} \mathrm{O}_{37} \mathrm{H}_{30}(1199 \mathrm{AO}$ basis functions and $2818 \mathrm{CD}$ fitting functions), using up to 70 nodes of the KSR-2.

\section{Collaboration in Molecular Science Software: Daresbury Laboratory - Pacific Northwest National Laboratory}

The Theory and Computational Science Division at the UK'S EPSRC Daresbury Laboratory and the EMSL both have a vital interest in the scientific application of high performance computing, especially parallel computing. Both groups aim to produce the next generation of scientific software in selected fields, and have established a full collaborative program of work with the focus on three broad areas of computational science:

- the electronic structure of molecules which includes the Advanced Correlated Methods and Hybrid Quantum Mechanical/Molecular Mechanical Methods

- the electronic structure of periodic solids which includes the Parallelization of CRYSTAL92, and
- molecular dynamics simulation which includes the Implementation of Fast Multipole Method and the Implementation of Cell Multipole Method.

A series of staff visits between the groups has been established, with the aim of sharing expertise and avoiding duplication, ensuring wide and early access to the latest hardware and software (both scientific applications codes and programming tools), and raising the profile of novel computational science in the U.S. and in Europe.

A major focus of the collaboration involves the integration and exploitation of the parallel tools developed in the EMSL into a variety of application codes. The distributed global array tools lie at the heart of the collaboration, allowing for the development of chemistry software that is fully distributed (i.e., the per processor memory requirements are minimized), and large quantities are distributed across the global memory of the machine. This removes the replicated data structure which was prevalent in many early parallel 
chemistry implementations, allowing us to reduce node memory requirements, address much larger problems, and to efficiently exploit machines with many more processors. Much of the development work at Daresbury is now focused on the Cray T3D, following the procurement of a 300-node machine at the University of Edinburgh. The recent port of the GA Tools to that platform has been an important development for the collaboration. We summarize below progress to date in each of the areas referred to above.

Advanced Correlated Methods (D. E. Bernholdt, $^{(a)}$ R. J. Harrison, A. P. Rendell, ${ }^{(b)}$ and A. T. Wong ${ }^{(a)}$ )

(a) AWU Postdoctoral Research Associate

(b) EPSRC Daresbury Laboratory, Daresbury, UK

The period from 1994-1995 saw the variety of tools developed in the EMSL come into regular use at Daresbury, particularly in the development of the coupled cluster (CC) code that is soon to be integrated under the NWChem umbrella. This code performs single and double excitations from a closed-shell reference and includes the effects of triple excitations using perturbation theory, viz coupled cluster with singles, doubles, and iterative triples $[\operatorname{CcsD}(T)]$. Given the computational cost of the method, implementation will be limited to systems containing approximately 500 basis functions.

Dr. Rendell visited PNNL from the October 6-14, 1994. During this time he presented a seminar covering a variety of aspects of the massively parallel quantum chemistry development work under way at Daresbury. In particular, new methods for converging SCF wave functions that avoid using a diagonalization, the development of a parallel MP2 gradient code, and the use of approximate integrals in coupled-cluster calculations.

The talk highlighted a mutual interest in parallel direct four-index transformations, particularly the transformations required to produce the limited Coulomb and Exchange operator matrices which are needed in many direct correlated calculations. During the remainder of his visit, Dr. Rendell worked in close collaboration with Drs. Wong and Harrison implementing a prototype direct transformation code into NWChem. Following this initial work, significant further progress has been made by Dr. Wong and the latest parallel direct transformation code in NWChem has shown an efficiency of over $90 \%$ for direct MP2 calculations on morphine using a 6-31G basis and performed on the Intel Delta.

In addition to the work performed above, 1994 saw the establishment of a collaborative project involving PNNL, DL, and Cray Research. The initial aim of this project was to port NWChem to the Cray T3D, optimize the code, and perform demonstration calculations. A longer-term aim will be to develop additional functionality within NWChem, including the development of gradient methods for post Hartree-Fock wave functions, and the evaluation of excitations energies using CC response theory. Specifically, we will develop an efficient $\operatorname{CCSD}(\mathrm{T})$ gradient program to complement the $\operatorname{CCSD}(\mathrm{T})$ energy code already developed. The code will have similar storage requirements to the energy program and should therefore be capable of performing calculations on systems containing approximately 500 basis functions. Targeting systems of such size will inevitably necessitate the investigation of direct technology in a gradient framework, a matter which has not been considered beyond the MP2 level. The CCSD $(T)$ gradient requires a variety of steps that will require extensive modifications to run in parallel, and substantial re-design if we accept that for large systems we cannot store four index quantities containing three or four virtual indices. This project formally started in January 1995 when the associated research assistance, Dr. Kobayashi, took up her position.

Hybrid Quantum Mechanics/Molecular Mechanics Methods (M. F. Guest, X. Long, ${ }^{(a)} \mathrm{J}$. B. Nicholas, and P. Sherwood ${ }^{(b)}$ )

(a) AWU Postdoctoral Research Associate

(b) EPSRC Daresbury Laboratory, Daresbury, UK

$A b$ initio quantum mechanics (QM) calculations can give highly accurate results. However, due to their high computational cost, they are customarily applied to only small molecules and clusters (less than 50 atoms). In contrast, molecular mechanics (MM) calculations are much cheaper and are readily applied to very large systems $(\approx 100,000$ atoms) such as hydrated pro- 
teins. MM methods are based on a classical force field, which represents inter- and intramolecular interactions by simple functions, with the required force field parameters derived by comparison to experimental data. The low cost of MM methods allows the study of the time evolution of these large systems by integration of the equations of motion in MD simulations. While MM methods are computationally efficient, they are not able to adequately describe chemical phenomena, such as the breaking and forming of bonds, which are required to model reactions. In order to make tractable the study of the chemical behavior of large systems, it is possible to combine features of both the QM and MM methods. ${ }^{41-49}$ Thus, small parts of the systems that require a rigorous description are treated quantum mechanically, while the majority of the system is described classically.

The basic idea is to partition the system into a $\mathrm{QM}$ region and a MM region. A diagram of this partition for sulfuric acid in water is shown in Figure 4.9. Under this partition, the effective Hamiltonian $\left(H_{\text {Eff }}\right)$ of a system can be written as

$$
\mathrm{H}_{\mathrm{eff}}=\mathrm{H}_{\mathrm{QM}}+\mathrm{H}_{\mathrm{MM}}+\mathrm{H}_{\mathrm{QM} / \mathrm{MM}}
$$

in which $H_{\mathrm{QM}}$ describes the $\mathrm{QM}$ atoms, $H_{\mathrm{MM}}$ gives the molecular mechanical force field for the $\mathrm{MM}$ atoms, and $H_{\mathrm{OM} / \mathrm{MM}}$ is the Hamiltonian that couples the QM and MM atoms.

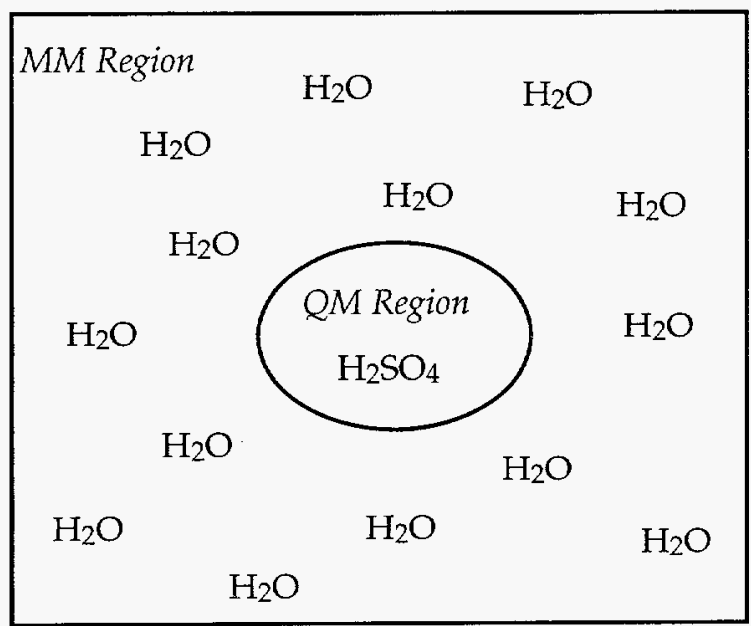

Figure 4.9. The diagram of one possible $\mathrm{QM} / \mathrm{MM}$ system partition for sulfuric acid in a box of water.

$$
\begin{aligned}
& \mathrm{H}_{\mathrm{QM} / \mathrm{MM}}=-\sum_{\mathrm{iM}} \frac{\mathrm{q}_{\mathrm{M}}}{\mathrm{r}_{\mathrm{iM}}} \\
& +\sum_{\sigma \mathrm{M}}\left[\frac{\mathrm{Z}_{\mathrm{S}} \mathrm{q}_{\mathrm{M}}}{\mathrm{R}_{\sigma \mathrm{M}}}+\frac{\mathrm{B}_{\sigma \mathrm{M}}}{\mathrm{R}_{\sigma \mathrm{M}}^{12}}-\frac{\mathrm{A}_{\sigma \mathrm{M}}}{\mathrm{R}_{\sigma \mathrm{M}}^{6}}\right]
\end{aligned}
$$

The terms in $\mathrm{H}_{\mathrm{QM}} / \mathrm{MM}$ represent electron-MM partial charge interactions, QM/MM nuclearnuclear interactions, and long-range dispersion effects.

The total energy of the system is the expectation value of the wave function for $\mathrm{H}_{\mathrm{Eff}}$ :

$$
\mathrm{E}_{\mathrm{Tot}}=\left\langle\Psi\left|\mathrm{H}_{\mathrm{Eff}}\right| \Psi\right\rangle \text {. }
$$

The forces on QM and MM atoms, needed for geometry optimizations and dynamic simulations, are $\mathrm{F}_{\mathrm{QM}}=-\mathrm{dE}_{\mathrm{Tot}} / \mathrm{dR}_{\mathrm{QM}}$ and $\mathrm{F}_{\mathrm{MM}}=-\mathrm{dE}_{\mathrm{Tot}} /$ $\mathrm{dR}_{\mathrm{MM}}$. It is obvious from the above that the only additional quantum calculations involved are the one-electron integrals between the MM partial charges and the electronic wave function of $\mathrm{QM}$ atoms. Many variations of the combined $\mathrm{QM} / \mathrm{MM}$ method are possible, the key differences being the manner in which the $Q M$ and $\mathrm{MM}$ regions are described. For example, the $\mathrm{QM}$ region can be described with a semiempirical (SE) method, a Hartree-Fock or correlated method, or DFT. The MM region can be described by the empirical force field approach described above, continuum models, frozen density methods, or local orbital methods.

We have developed prototypes of both the SE/MM and DFT/MM formalisms. The prototypes integrate our parallel MD program with the semiempirical code MOPAC6 and the density functional code DeFT. We are currently using serial versions of both MOPAC ${ }^{50}$ and DeFT. ${ }^{51}$ The prototype codes already allow us to apply our extensive MD simulation capabilities to a wide range of problems, such as the study of catalysis by enzymes and zeolites, solvation dynamics, and reactions in solution. The use of the parallel, hierarchical CMM (described above) for the rapid evaluation of long range classical electrostatics allows us to treat systems containing as many as a million classical atoms with the $\mathrm{QM} / \mathrm{MM}$ method. We are currently integrating the SCF and DFT modules of NWChem with the MD code. The use of the parallel SCF and DFT 
codes will greatly expand the possible size of the $Q M$ region in the $Q M / M M$ method.

Electronic Structure of Periodic Solids: The Parallelization of CRYSTAL92 (E. Aprà ${ }^{(a)}$ and V. R. Saunders $(b)$ )

(a) AWU Postdoctoral Research Associate

(b) EPSRC Daresbury Laboratory, Daresbury, UK

The periodic Hartree-Fock code, CRYSTAL, ${ }^{52-54}$ has been parallelized initially to use message passing with a replicated data approach. Both the PVM3.2 $2^{55}$ and TCGMSG ${ }^{1}$ message-passing libraries can be accommodated. Parallelization has been accomplished for computation of the x-ray structure factors, electron momentum maps, and Compton profiles; and for the post-SCF computation of crystalline properties (e.g., properties connected with the eigenvalues $\mathrm{e}(\mathrm{k})$ : density of states and band structure).

The parallel performance of CRYSTAL has been improved by reducing the amount of $\mathrm{I} / \mathrm{O}$ and by introducing the GA tools. Reduced $\mathrm{I} / \mathrm{O}$ is achieved by using the direct SCF version of the code and by using the dynamic MA to store quantities such as eigenvectors and one-electron integrals. Introduction of the GA tools provides such functionalities as parallel matrix eigensolvers and multipliers; the aim being to distribute the diagonalization process at a single point among multiple nodes instead of distributing the work over k-points. This should be exploited for systems where the Fock matrix has large dimensionality and where computational accuracy can be achieved using few k-points.

Future developments include moving to a completely "distributed data" version, where the largest data structures (Fock and density matrices) will be distributed among the nodes, instead of each node having its own copies. This approach will dramatically decrease the amount of memory needed and, therefore, increase the size of the problems amenable to treatment. The most important steps to consider are 1) the calculation of the Fock matrix, $\mathrm{F}_{\mathrm{mn} ; \mathrm{g}^{\prime}}$ in direct space by using the density matrix $\mathrm{P}_{\mathrm{mn} ; \mathrm{g}}$ and the 2-electron integrals; and 2) the Fourier transform of the Fock matrix from direct to reciprocal space: $F_{m n ; g} \rightarrow$ $\mathrm{F}_{\mathrm{mn}}(\mathrm{k})$.
Preliminary results on the KSR-2 are tabulated in Table 4.1 for a direct-SCF calculation of the Hartree-Fock wave function of a molecular crystal of urea. In this calculation there were 160 AOs, 58 k-points, and $5.5 \times 10^{6}$ one-electron integrals. For the same test on a single processor Cray C-90, the CPU time was 7932 seconds.

Molecular Dynamics Simulations (R. Kutteh, ${ }^{(a)}$ J. B. Nicholas, and W. Smith ${ }^{(b)}$ )

(a) AWU Postdoctoral Research Associate

(b) EPSRC Daresbury Laboratory, Daresbury, UK

We are developing a full-featured, generalpurpose module within NWChem for MD simulations on MPPs, designed to treat virtually all chemical systems, with the same functionality as many commercial programs. The code features an extensive and easily modifiable force field, efficient treatment of long range interactions via Ewald summations and fast multipole methods, a variety of statistical mechanical ensembles, energy minimizations, and normal mode calculations. The code runs on a wide range of MPPs and gives large performmance gains over scalar and vector computers. For example, on 512 processors of the Intel Delta, our code requires less than a tenth of a second to perform a step of MD on 7776 atoms. We have performed some large MD simulations studying diffusion in zeolites to better understand separation processes in these materials. These nanosecond simulations involved $\approx 10,000$ atoms and required $\approx 100$ hours on the Intel Delta. We are developing a distributed data version of this code that will extend the size of systems that can be

Table 4.1. KSR-2 wall clock times and efficiencies for periodic direct-SCF calculations on the molecular crystal of urea.

\begin{tabular}{ccc}
$\begin{array}{c}\text { Number } \\
\text { of Nodes }\end{array}$ & $\begin{array}{c}\text { Wall Clock } \\
\text { Time }\end{array}$ & Efficiency \\
\hline 1 & 19426.4 & 1.000 \\
4 & 4971.1 & 0.977 \\
8 & 2622.3 & 0.926 \\
16 & 1482.5 & 0.819 \\
24 & 1115.8 & 0.725 \\
32 & 884.8 & 0.686 \\
40 & 785.7 & 0.618 \\
48 & 798.7 & 0.507
\end{tabular}


studied to beyond a million atoms.

The major long-range interactions in MD simulations are Coulombic and dipolar, and are the most expensive to compute, often accounting for $90 \%$ or more of the total computation time. Typically, MD simulations are performed with periodic boundary conditions imposed on the simulation box, and the traditional methods of computing the forces involve the use of either minimum image or Ewald techniques.

For a system of $\mathrm{N}$ particles, minimum image, in conjunction with spherical cut-off and some bookkeeping methods, 56 reduces the computation time to $\mathrm{O}(\mathrm{N})$. However, minimum image is more adequate for short-range forces, such as dispersion and van der Waals, typically modeled by a Lennard-Jones potential; for the long-range charge and dipole interactions, minimum image is generally inaccurate. 57,58 These long-range forces are therefore usually handled using Ewald or Ewald-Kornfeld methods. ${ }^{59}$ While accurate, these Ewald techniques can have large computational requirements. Similar difficulties arise in computing the long-range forces in a finite system (i.e., vacuum or free space boundary conditions). For a finite system of $\mathrm{N}$ charges or dipoles, direct calculation of electrostatic forces scales as $\mathrm{N}(\mathrm{N}-1) / 2$. This approach leads to prohibitively high computational costs for systems of more than a few thousand particles and is not feasible for very large systems. On the other hand, potential truncation methods, like their periodic counterparts, ignore long-range tails and are generally inaccurate.

In the recent past, "hierarchical codes" or "tree codes" have been developed in astrophysics ${ }^{60-62}$ to efficiently compute Coulomb-type sums. Unlike particle-mesh methods, ${ }^{63}$ tree codes are gridless and instead divide the computational region into a hierarchy of cells, where cells at successive levels of refinement form a tree structure (i.e., subdivision of a cell at some level leads to children cells at the next level of refinement). The mass and center of mass of each cell are computed at one level and used in a monopole approximation to compute the interaction between every particle and its cell's interaction list (the interaction list of a cell consists of the children of the near neighbors of its parent which are not near neighbors of the cell itself). The algorithm then recursively performs the same sequence of steps on neighboring cells at the next level of refinement. These algorithms are of order $\mathrm{O}(\mathrm{N} \log$ $N$ ) in computation time, but limited in accuracy.

Improvements to these algorithms, including the addition of higher terms in the expansion (asymptotic expansion), ${ }^{64}$ the introduction of Taylor expansions at observer sites, and the shifting of multipole and Taylor expansions between levels (eliminating costly recomputation at every level, as for multipole expansions in tree codes) culminated in the $\mathrm{O}(\mathrm{N})$ fast multipole method (FMM) of Greengard and Rokhlin. 65,66 Work on the implementation of FMM is currently under way in both the EMSL and Daresbury Laboratory.

\section{The Implementation of FMM}

The computation of long-range (Coulombic) forces in classical molecular simulation is an expensive task in comparison with the short-ranged van der Waals forces. For a finite system (i.e., free space or vacuum boundary conditions), exact evaluation of either type of forces exhibits a computational cost scaling as $\mathrm{N}^{2}$, where $\mathrm{N}$ is the number of charged atoms in the simulated system. However, the short range of the van der Waals forces forces allows the adoption of efficient cutoff strategies which computationally scale as $\mathrm{N}$. Unfortunately, the long range of Coulomb interactions prevents their accurate computation using cutoff techniques.

For a periodic system (or pseudo-periodic), the long range of Coulombic forces means that they are only conditionally convergent. Hence, as in the finite system case, periodic cutoff methods like minimum image which reduce the computational cost of van der Waals forces forces to $O(N)$ (with some bookkeeping), are generally inaccurate for Coulombic interactions. For this reason the method of choice in much of molecular simulation is the Ewald method, which guarantees convergence and offers $\mathrm{N}^{3 / 2}$ scaling. The Ewald method is also highly vectorizable and very efficient codes exist for its evaluation. Despite the improved scaling and high efficiency however, the method still becomes prohibitively expensive for large systems of the order of $10^{4}$ atoms and above.

As discussed above, recently a number of efficient methods for evaluating Coulomb sums in 
finite systems with $\mathrm{O}(\mathrm{N})$ scaling (instead of $\mathrm{N}^{2}$ ), have appeared. These are the so-called fast multipole methods, which through a combination of elegant expansion and shift theorems in a hierarchical computational strategy, allow effects of distant clusters of charges (represented as electrostatic multipoles) to be evaluated with a greatly reduced $\mathrm{N}$ dependence. For periodic systems, the fast multipole methods are coupled with the Ewald approach to handle the conditional convergence problem. For large-scale simulations, these combined fast multipole/Ewald algorithms will greatly out-perform the traditional Ewald method.

In this collaboration between Daresbury Laboratory and the EMSL, we are studying the three-dimensional FMM of Greengard and Rokhlin for possible replacement of the Ewald sum in large-scale serial and parallel simulations. A FMM code has been written and is being used to study several aspects of the method. The first of these is computational efficiency. At a naive level, the method is difficult to code in an efficient manner. A particularly important (possibly crucial) aspect of the efficiency is the high complexity of the method, leading to a high computational cost which is only offset when the simulations become extremely large. The question of when the $\mathrm{N}$ dependence of the method finally out-performs the Ewald $\mathrm{N}^{3 / 2}$ is currently a subject of great debate, even controversy. Our own work suggests that the crossover point is of the order $10^{4}$ to $10^{5}$ atoms.

The scaling issue is deeply embedded in the question of accuracy, which is the second aspect of this study. The accuracy of the FMM method is dependent on two parameters: the order (p) of the multipole expansion; and the level $\left(l_{\max }\right)$ of the spatial hierarchy, the first of these being the most important. Choosing either of these parameters has a significant effect on the computational cost of the method. The initially published estimates for the accuracy of the FMM method are now thought to be overly pessimistic. The question now is to determine what values of $p$ and $l_{\text {max }}$ optimize performance without jeopardizing the accuracy.

The third aspect of this work is the parallelization of the method. Efficient methods for parallelizing the Ewald sum are known and are currently being used. The most logical possibility for paral- lelizing the FMM appears to be domain decomposition, which has similar geometrical requirements to FMM. However, the inter-node communication strategy in domain decomposition is local, whereas FMM will probably require nonlocal communications, with consequences for overall scaling with the number of nodes.

\section{The Implementation of CMM}

The formulation of three-dimensional FMM ${ }^{66}$ is couched in spherical harmonics language. Recently, however, a Cartesian version of FMM, the cell multipole method (CMM), was proposed by Ding, Karasawa, and Goddard. ${ }^{67}$ Both approaches use a tree structure where a cell at level (l) is divided into eight children cells at level $(1+1)$, each of which is divided in turn into eight children cells at level (1+2), and so on.

The algorithm consists of an upward pass (finer to coarser levels) during which the multipole moments are computed, and a downward pass (coarser to finer levels) during which the Taylor coefficients are computed. At the finest level, the near interactions are computed directly and the far interactions are obtained from the Taylor expansions. Both contributions are summed to get the total energy and forces.

CMM is physically more transparent than FMM, is easier to implement, and can be incorporated naturally into various MD calculations which are typically set in Cartesian coordinates. In addition, CMM is more amenable to extension to other than charge-charge interactions (e.g., to any polarizable charged dipolar system). ${ }^{68,69}$ In addition to rapidly computing all of the interactions in such a polarizable system, this extended algorithm performs sufficient self-consistent iteration of the induced dipoles. In our implementation of FMM discussed above, a desirable accuracy can be achieved by retaining a high enough number of terms in the expansions. In CMM, increased accuracy requires the explicit addition of higher terms in the expansions. The relative complexity of Cartesian expansion coefficients and the considerable effort required to program them impose a practical limit on the attainable accuracy of CMM. These drawbacks, however, do not seriously limit the use of CMM, since in practice very good accuracy is obtained with only the first few orders $^{66,68,69}$ Figure 4.10 shows the speedup obtained on the Intel Touchstone Delta for the 
Coulomb energy and forces computations, using our third order CMM implementation (with finest level 4), in a simulation on a random collection of 23,375 charged particles (i.e., with 5.7 particles per cell on average).

Fast multipole methods such as FMM and CMM exploit the slow variation of the far part of longrange interactions over particle coordinates. They consequently approximate the direct computation of the far part with more efficient multipole and Taylor expansions. In contrast to these spatial approximations, multiple time step methods (MTS) ${ }^{70,71}$ exploit the slow variation of far interactions over time in MD simulations. In MTS, the force on a particle is divided into a rapidly changing primary component due to nearest neighbor particles, and a slowly changing secondary component due to more remote neighbor particles. The idea then is to compute the primary forces every time step dt and the secondary forces every ndt. Between $t+d t$ and $t+$ $\mathrm{ndt}$, the secondary forces are propagated through a Taylor expansion. The method then effectively uses two time steps: $\mathrm{dt}$ for primary forces and ndt for secondary forces (it is also possible to use more than two time steps). The same idea can be applied to interactions computed with the fast multipole methods in MD simulations. The directly-calculated near part is computed every time step. The expansion-calculated far part is computed less frequently. Effectively, a larger time step is used to compute the Taylor coefficients of the potential expansion. Between their successive computations, the Taylor coefficients are propagated through a Taylor expansion in

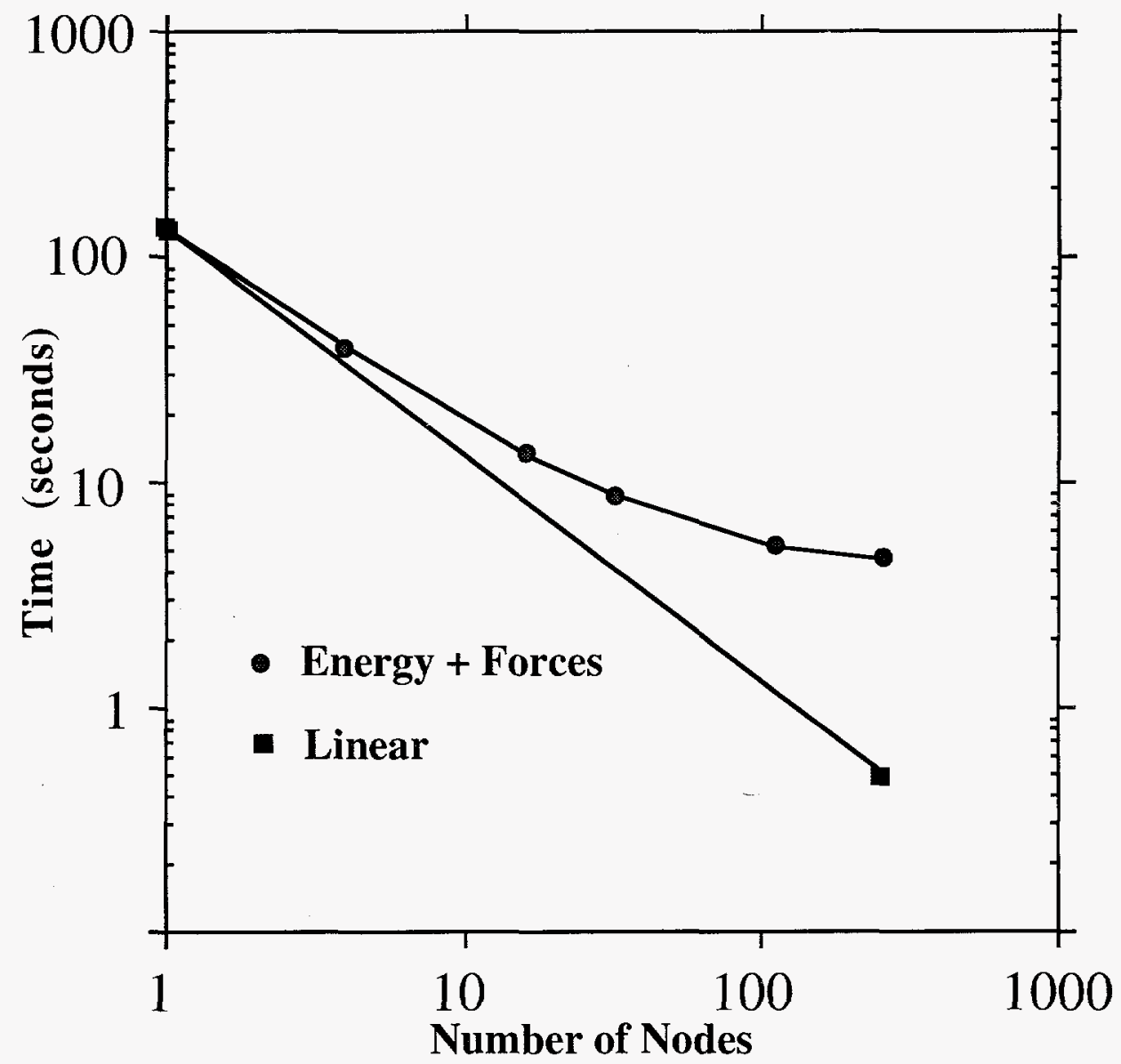

Figure 4.10. Speed up on the Intel Touchstone Delta for the Coulomb energy and forces computation, using third order CMM with finest level 4, for a random collection of 23,375 charged particles (i.e., about 5.7 particles per cell at the finest level). 
time. When incorporated into FMM or CMM, this MTS algorithm should lead to a substantial increase in efficiency.

\section{Applications to Prototypical or Model Problems in Chemistry}

A large component of software development includes the demonstration or utilization of the software on model problems. In some cases (for example, DFT) the methods have to be explored to determine their applicability to the environmental chemistry problems at hand. Many times these applications are performed in collaboration with groups that are on site (for example, in collaboration with the Biomolecular Modeling and Simulation group or the Solid State Theory group). In addition, there are a wide range of ongoing collaborations in computational chemistry methods and applications which include interactions with over 60 scientists at institutions in the U. S. and Europe. These collaborations/ interactions have been very productive and a select few of these are highlighted below.

An Extended Basis Set $A b$ Initio Study of $\mathrm{Li}^{+}(\mathrm{H} 20)_{\mathrm{n}}, \mathrm{n}=1-6$ (D. F. Feller, ${ }^{(a)}$ E. D. Glendening, ${ }^{(b)}$ R. A. Kendall, and K. A. Peterson $^{(c)}$ )

(a) Computational Chemistry Environment Group

(b) AWU Postdoctoral Research Associate, Molecular Theory Group

(c) Joint appointment with Molecular Theory Group and Department of Chemistry, Washington State University

The structures, binding energies, and enthalpies of small molecular clusters incorporating a single lithium cation and up through six waters have been determined with extended Gaussian basis sets using Hartree-Fock and post-Hartree-Fock methods. The resulting properties were analyzed with respect to both basis set completeness and degree of correlation recovery, including corecore and core-valence effects. Although the lithium-water interaction is largely electrostatic in nature, small basis sets, lacking in polarization and near-valence diffuse functions, drastically overestimate the strength of the bond (by 20 $\mathrm{kcal} / \mathrm{mol}$ or more) and underestimate the $\mathrm{Li}^{+} \ldots \mathrm{O}$ distance by up to $0.1 \AA$. Their poor performance is attributable to inherent errors in describing the electric moments and polarizability of water and to large basis set superposition errors. Thus, the accuracy with which the fundamental lithiumwater interaction could be modeled was primarily dependent on the quality of the Gaussian basis set and not upon the level of correlation recovery. Basis set enlargement and correlation effects both tend to reduce the strength of the $\mathrm{Li}^{+}\left(\mathrm{H}_{2} \mathrm{O}\right)$ bond, but produce corrections of opposite sign for the $\mathrm{Li}^{+\ldots} \mathrm{O}$ bond length. Although correlation effects play a minor role in describing the lithium-water interaction, as the size of the cluster increases and the number of waters involved in multiple hydrogen bonds grows, correlation recovery can become significant.

Theoretical Studies of Inorganic and Organometallic Reaction Mechanisms: Hydrogen Exchange in the B-agostic Ethylene Complex of Cyclopentadienyl Rhodium (M. F. Guest, M. B. Hall, (a) Z. Lin, (a) and P. Sherwood ${ }^{(b)}$ )

(a) Chemistry Department, Texas A\&M University

(b) EPSRC Daresbury Laboratory, Daresbury, UK

$A b$ initio calculations with effective core potentials have been used to study the hydrogen exchange process of the $\left[\mathrm{CpRh}\left(\mathrm{C}_{2} \mathrm{H}_{4}\right)\left(\mathrm{n}^{2} \mathrm{C}_{2} \mathrm{H}_{5}\right)\right]^{+} \mathrm{B}-$ agostic complex. The mirror-symmetric olefinhydride species is found to be an intermediate $(3.4 \mathrm{kcal} / \mathrm{mol}$ higher in energy than the B-agostic complex) rather than a transition state (ca. 5 $\mathrm{kcal} / \mathrm{mol}$ ) in the interconversion process of the two enantiomeric forms. A higher energy process involving rotation of the methyl group in the agostic complex is determined to occur through an $\mathrm{Rh} . . .\left(\mathrm{n}^{2-} \mathrm{H}_{2} \mathrm{C}\right)$ interaction with a calculated activation energy of ca. $6.5 \mathrm{kcal} / \mathrm{mol}$. Complete loss of the $\mathrm{Rh} . . . \mathrm{H}-\mathrm{C}$ agostic interaction requires an activation energy of about $14 \mathrm{kcal} / \mathrm{mol}$. This agostic interaction strength depends upon the $\mathrm{M}$...H overlap, and stronger agostic interactions result in weaker $\mathrm{C}-\mathrm{H}_{\text {agostic }}$ bonds. An even higher energy process involving the exchange of all of the nine hydrogens and the four carbons is found to be due to the ethylene rotation in the agostic complex. The ethylene rotation barrier (calculated to be $9.8 \mathrm{kcal} / \mathrm{mol}$ ) depends greatly on the sizes of the transition metal atom and ligands in the complex since steric effects play an important role in the rotation. 
Models for the Molybdenum (VI/V) Centers of the Molybdenum Hydroxylases and Related Enzymes. Geometry, Electronic Structure, and Electron Paramagnetic Resonance g-Tensor Predictions from $A b$ Initio and Zindo Molecular Orbital Studies (E. McCullough, ${ }^{(a)}$ J. A. Nichols, G. Peng, ${ }^{(a)}$ and J. Spence $\left.{ }^{(a)}\right)$

(a) Chemistry Department, Utah State University

The geometries, electronic structures, and electron paramagnetic resonance (EPR) g-tensors for several molybdenum(V) and (VI) complexes with tetradentate $\mathrm{N}_{2} \mathrm{~S}_{2}$ and $\mathrm{N}_{2} \mathrm{O}_{2}$ ligands $\left[\mathrm{LH}_{2}=\mathrm{N}\right.$, $\mathrm{N}^{\prime}$-bis(2-mercapto-phenyl)- $\mathrm{N}, \mathrm{N}^{\prime}$-dimethyl-1,2diaminoethane; $\mathrm{N}, \mathrm{N}^{\prime}$-bis(2-hydroxyphenyl)-N, $\mathrm{N}^{\prime}$-dimethyl-1,2-diaminoethane] were predicted using $a b$ initio and INDO molecular orbital methods. These models have been proposed as models for the molybdenum(VI/V) centers of the molybdenum hydroxylases and related enzymes. $A b$ initio and INDO geometrical predictions were compared with known x-ray crystal structures for three complexes and were found to be in excellent agreement. The results support previously proposed structures for both the model complexes and the enzyme molybdenum centers and are in good agreement with EPR and extended $x$-ray absorption fine structure (EXAFS) experimental data for these systems. The results indicate the ligand has a profound effect on the geometry and electronic structures of the complexes. Insight into the electronic factors responsible for the EPR parameters of the complexes was found and application to the enzyme molybdenum centers was explored.

Benchmark Calculations with Correlated Molecular Wave Functions. III. Configuration Interaction Calculations on First Row Homonuclear Diatomics (K. A. Peterson, ${ }^{(a)}$ R. A. Kendall, and T. H. Dunning, Jr.)

(a) Joint appointment with Molecular Theory Group and Department of Chemistry, Washington State University

Using correlation consistent basis sets from double through quintuple zeta quality, ${ }^{72-74}$ potential energy functions have been calculated for the electronic ground states of the first row homonuclear diatomic molecules $\mathrm{B}_{2}, \mathrm{C}_{2}, \mathrm{~N}_{2}, \mathrm{O}_{2}$, and $\mathrm{F}_{2}$ using single and double excitation configuration interaction (Hartree-Fock $+1+2, \mathrm{GVB}+1+2$, and
CAS $+1+2)$ wave functions. Spectroscopic constants have been calculated for each species and compared to experiment. The dependence of the calculated spectroscopic constants on systematic extensions of the one-particle basis set are, in general, found to be very regular. By fitting the directly calculated values with a simple exponential function, accurate estimates of the complete basis set (CBS) limit for $\mathrm{E}_{\mathrm{e}^{\prime}} \mathrm{D}_{\mathrm{e}^{\prime}}$ and $\mathrm{r}_{\mathrm{e}^{\prime}}$ have been obtained for each level of theory. The estimated CBS limits were compared to the available experimental results, and the intrinsic errors associated with each theoretical method were characterized. In addition the accuracy of the internally contracted CAS $+1+2$ method was compared to conventional uncontracted calculations using large basis sets. For $\mathrm{B}_{2}$, a full configuration interface calculation has been carried out for $\mathrm{D}_{e}$ with the correlation consistent double zeta basis set and was compared to the CAS $+1+2$ method using both a supermolecule and separated atom approach for the dissociated limit.

Ionization Potentials, Electron Affinities, and Potential Energy Curves Determined from the Multiconfigurational Spin Tensor Electron Propagator Method (MCSTEP) (J. T. Golab, ${ }^{(a)}$ D. Heryadi, ${ }^{(b)}$ J. A. Nichols, and D. L. Yeager $\left.{ }^{(b)}\right)$

(a) Amoco Research

(b) Department of Chemistry, Texas A\&M University

With electron propagator methods, electronic ionization and attachment energies are obtained directly. The MCSTEP method is explicitly designed for systems with open shell and/or nondynamical correlation in the initial state. We performed several studies in order to assess the strengths and weaknesses of this method compared to other accepted theories. ${ }^{75-78}$

\section{A Comparison of the MCSTEP with Extended Koopmans' Theorem Results}

We applied the MCSTEP to determine the lowest few (in energy) vertical ionization potentials (IPs) of $\mathrm{HF}, \mathrm{H}_{2} \mathrm{O}, \mathrm{NH}_{3}, \mathrm{CH}_{4}, \mathrm{~N}_{2}, \mathrm{CO}, \mathrm{HNC}, \mathrm{HCN}$, $\mathrm{C}_{2} \mathrm{H}_{2}, \mathrm{H}_{2} \mathrm{CO}$, and $\mathrm{B}_{2} \mathrm{H}_{6}$. We chose these molecules so that we could compare MCSTEP IPs with recently reported extended Koopmans' theorem (EKT) IPs on the same molecules. Using standard Dunning core-valence basis sets 72 with relatively small complete active spaces, MCSTEP 
results are in very good to excellent agreement with experiment. These MCSTEP IPs are obtained using matrices no larger that $400 \times 400$. To obtain fairly accurate EKT results, large active spaces are required. Our calculations showed that MCSTEP IPs obtained using a small complete active space are usually better than the EKT result, particularly for the low-lying IPs.

A Comparison of the MCSTEP with "Benchmark" Full Configuration Interaction and Large-Scale Multireference Configuration Interaction Calculations

Using the same basis sets and geometries as were previously used in "benchmark" full configuration interaction ( $\mathrm{FCI}$ ) calculations, we compared the MCSTEP with FCI for the vertical IPs in $\mathrm{CH}_{2}$ below $19.0 \mathrm{eV}$. Our results show that MCSTEP using a full valence complete active space MCSCF initial state accurately obtains the lowest several principal vertical ionization potentials. We also determined vertical and adiabatic IPs in $\mathrm{CH}_{2}$ with MCSTEP using larger bases and compared to accurate large-scale multireference singles and doubles $\mathrm{CI}$ with quadruple excitations estimated via a Davidson correction. Our adiabatic MCSTEP IPs are generally in excellent agreement with the $\triangle \mathrm{MRSDCI}+\mathrm{Q}$ adiabatic IPs with about the same agreement as obtained for MCSTEP compared to the vertical $\triangle F C I$ IPs in the DZP basis.

A Comparison of the MCSTEP with the CoupledCluster Reference Electron Propagator Method and the Fock Space Multireference CoupledCluster Method

Here, we performed vertical ionization potential calculations on $\mathrm{O}_{2}$ using exactly the same basis sets and geometries that were reported from the application of two other forefront methods: the coupled-cluster reference electron propagator method (CC-EPT) and the Fock space multireference coupled-cluster method (FRMRCC). All of these methods are apparently very accurate and reliable for determining vertical ionization potentials. MCSTEP at this time may have some advantages in being slightly less sensitive to basis set variations (provided, of course, that the basis sets are at least of double-zeta with polarization quality) and in being capable of determining accurate IPs for more states when the initial state is open shell.
Application of Combined Density Functional Theory/Molecular Mechanics to Small Water Clusters: A Test of Methodology (X. P. Long, $(a)$ J. B. Nicholas, M. F. Guest, and R. L. Ornstein ${ }^{(b)}$

(a) AWU Postdoctoral Research Associate

(b) Biomolecular Modeling and Simulation Group

We have tested our prototype DFT/MM by calculating the structure and interaction energies for a variety of water clusters, with different numbers of water molecules represented classically or quantum mechanically. We show the optimized geometry in Figure 4.11 for a water dimer in which one molecule is classical and the other one is described using the DFT formalism in DeFT, the mixed Becke-Perdew nonlocal functional, ${ }^{36,79}$ and the cc-pVDZ basis set. ${ }^{72,73}$ The geometry of the dimer in the mixed QM/MM formalism is in very good agreement with a full DFT calculation at the same level of theory. For example, the $\mathrm{O}-\mathrm{H}$ distance and $\mathrm{O}-\mathrm{H}-\mathrm{O}$ angle shown in Figure 4.11. agree exactly between the two calculations. The interaction energy between the two water molecules does not agree as well; it is 6.06 $\mathrm{kcal} / \mathrm{mol}$ with the $\mathrm{QM} / \mathrm{MM}$ method versus 5.43 $\mathrm{kcal} / \mathrm{mol}$ for the full DFT calculation. Further tests of the DFT/MM method are under way.

Molecular Mechanics Parameters for FluorineSubstituted Methanes from $A b$ Initio Quantum Calculations (J. L. Anchell ${ }^{(a)}$ and B. J. Palmer ${ }^{(b)}$ )

(a) Kendall Square Research Inc.

(b) Applied Mathematics, EMSL

Molecular mechanics parameters for the homologous series of fluorine-substituted methanes, $\mathrm{CH}_{\mathrm{x}} \mathrm{F}_{4-\mathrm{x}}$, were derived from $a b$ initio Hartree-Fock surfaces. $A b$ initio intermolecular potential energy surfaces were calculated using at the

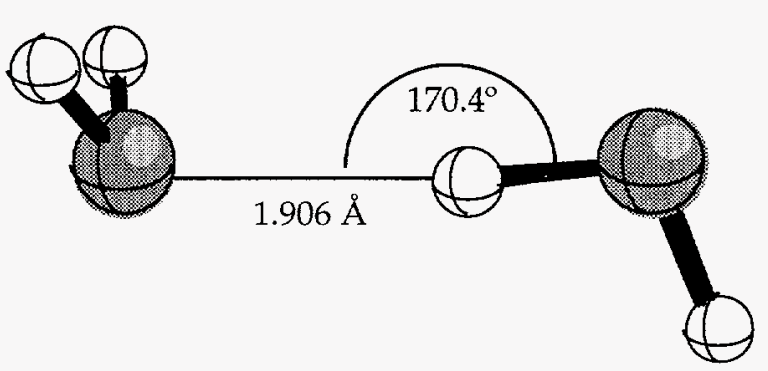

Figure 4.11. Optimized geometry of the water dimer from DFT calculations. 
$\mathrm{MP} 2 / 6-31+\mathrm{G}^{*}$ level of theory. A least squares fit of the $a b$ initio surface to a standard molecular mechanics potential function, including LennardJones interactions plus partial charges, was then performed. The thermodynamic properties of the resulting molecular mechanics potential were then calculated using conventional molecular dynamics simulations and compared to experiment. $^{80}$ Additional fine-tuning of the molecular mechanics potential was then performed to optimize agreement with experiment. The effect of including high energy configurations in the fit was systematically investigated.

Application of a Systematic Sequence of Basis Sets to the Nuclear Magnetic Resonance Shielding of $\mathrm{CH}_{4}$ and $\mathrm{SiH}_{4}$ (D. E. Bernholdt ${ }^{(a)}$ and J. B. Nicholas)

(a) AWU Postdoctoral Research Associate

NMR spectroscopy is an extremely versatile and important tool in chemistry. As with other spectroscopic methods, there is increasing interest in using the methods of computational chemistry to help analyze experimental spectra and for prediction of spectra for new chemical systems. Theoretical evaluation of NMR shielding parameters and chemical shifts is therefore of great practical interest, but at the same time offers significant challenges. In the zeolite ZSM- 5 there are 12 different chemical environments for the $\mathrm{Si}$ atoms which give rise to 12 peaks in the space of less than $10 \mathrm{ppm}$. We have undertaken a study of the basis set requirements for accurate prediction of NMR shielding constants in order to assess future prospects for their use in EMSL research.

To avoid the gauge origin problem common to many methods of computing magnetic properties, we use the gauge-invariant atomic orbital (GIAO, also known as London orbital) approach pioneered by Ditchfield. ${ }^{81}$ This approach was recently reformulated by Wolinski, Hinton, and Pulay ${ }^{82}$ in terms of analytic derivative techniques, which has greatly increased the efficiency of the method. In addition, Gauss ${ }^{83}$ has presented the GIAO-MP2 NMR equations, which make it possible to assess the effect of electron correlation on shieldings - a very significant contribution for some systems.
In this work we employ the "correlation consistent" basis sets developed by Dunning and coworkers. ${ }^{72-74}$ These basis sets (usually denoted cc-pVXZ, where $X=D, T, Q, 5$ ) are improved systematically as the size of the basis increases. Each successive set has a larger primitive space, a larger contracted set, and includes higher angular momentum functions. For example, the carbon sequence from double- to quintuple-zeta is 3s2p1d, 4s3p2d1f, 5s4p3d2f1g, and 6s5p4d3f2g1h contracted functions.

Experience with calculations on a variety of small molecules using the correlation consistent basis sets reveals a striking regularity in the properties calculated for a given system with basis sets of increasing size. This behavior, which has been observed for a wide range of properties including total and dissociation energies, equilibrium geometries, vibrational frequencies, and electrical polarizabilities and hyperpolarizabilities, usually manifests itself as an exponential convergence that can easily be extrapolated to obtain an estimate of the CBS result.

The initial phase of this work involved straightforward application of the correlation consistent basis sets to calculations on methane and silane at the MP2 level of theory. Our results for bondlength and total energy show the same sort of convergence which has been observed in previous studies. The heavy nuclei ( $\mathrm{C}$ and $\mathrm{Si}$ ) also display very regular behavior in the NMR shielding. The hydrogen shieldings are somewhat more complicated. The scalar isotropic values do not in general show the same convergent behavior as other properties. When individual tensor components are considered $(\mathrm{H}$ has two free elements in these molecules), most, but not all, do indeed behave quite regularly. The need to look at the individual components is not surprising since similar behavior has been observed for other properties such as the dissociation energy which are computed from the sum or difference of more basic quantities. The failure of some individual components to fit the exponential pattern deserves additional investigation, but theoretical modeling of proton NMR has historically been troublesome, so it may be a more complex problem.

Although both ${ }^{13} \mathrm{C}$ and ${ }^{29} \mathrm{Si}$ shieldings do show exponential convergence, the $\mathrm{Si}$ results showed a much larger variation from cc-pVDZ to cc-pVQZ. 
Consequently basis sets large enough to produce quality ${ }^{29} \mathrm{Si}$ shieldings rapidly outstrip the available computer facilities. This led to a second phase of this project: understanding the origin of this large basis set dependence and producing basis sets tailored to the efficient calculation of shielding tensors while retaining the systematic nature of the original correlation consistent sets. Initial results indicated that expansion of the core-valance region of the basis sets greatly improves the results and that diffuse functions have no effect. We are assessing the importance of higher angular momentum functions on the NMR shielding.

Weakly Bound van der Waals Complexes: The Interaction of Ar with NNO (H. A. Frücht $1,{ }^{(a)}$ R. A. Kendall, and A. T. Wong ${ }^{(\alpha)}$ )

(a) AWU Postdoctoral Research Associate

Geometry as well as vibrational and rotational modes of the van der Waals molecule Ar-NNO have recently been studied by S. Sharpe and coworkers using the infrared diode laser at PNNL. ${ }^{84}$ The cluster shows a slightly bent, Tshaped geometry, an anomalously high inertia defect, and a strong coupling between the highest-frequency intramolecular and the lowestfrequency intermolecular vibrations.

In order to verify their findings with $a b$ initio calculations, several methods and basis sets were tested. As the intermolecular bond is extremely weak a high level of correlation is required. Low levels of correlation (e.g., MP2) or poor basis sets give a binding energy which is too low and a distance between the monomers which is too long. On the other hand the basis set superposition error (BSSE), which tends to overestimate binding energies and give distances which are too short, has a major influence on the result, so that counterpoise correction has to be applied.

The method that was found to reproduce the experimental geometry best without requiring unreasonably high amounts of computation time or disk space is coupled cluster theory with estimated triples (CCSD $(T)$ ), using an augmented ccpvtz basis set for all atoms. Diffuse basis functions proved to be of very high importance because of the large distance $(3.5 \AA)$ between the argon atom and the center of the NNO molecule.
Figure 4.12 shows the energy surface of Ar in the field of a fixed NNO molecule. The lines of constant energy are determined via linear interpolation from the values at the grid points. The minimum at $3.47 \AA$ and $86^{\circ}$ exactly reproduces the experimental distance, while the angle is larger than the measured $81.4^{\circ}$. The surface is extremely flat, especially in angular direction. The binding energy at the minimum is $-0.83 \mathrm{mH}$.

Density Functional Calculations of Surface Defects on $\mathrm{MgO}(001)$ : Candidate Structures for Positive Ion Photo-Desorption (J. T. Dickinson, ${ }^{(a)}$ A. S. Gibson, ${ }^{(b)}$ J. P. Lafemina, ${ }^{(c)}$ J. A. Nichols, and H. L. Taylor ${ }^{(d)}$ )

(a) Department of Physics, Washington State University

(b) AWU Postdoctoral Research Associate, Advanced Processing Directorate, EMSL

(c) Advanced Processing Directorate, EMSL

(d) AWU Postdoctoral Research Associate

To further explore the performance and applicability of our NWChem DFT code, we are conducting a study of the $\mathrm{MgO}$ surface, as modeled by a $2 \times 3 \times 4$-atom cluster. Experiments have shown the desorption of positive ions, principally $\mathrm{Mg}^{+}$, from $\mathrm{MgO}$ surfaces during sub-bandgap $248 \mathrm{~nm}$ irradiation, to be accompanied by photo-electron emission. The ion emission is highly collimated along the surface normal, and exhibits a complicated energy dependence. Anion vacancy defects (F-centers) in the surface region, readily introduced by polishing, are necessary for this emission.

To understand this desorption process, a comparison is made between two models of cation emission from the (001) surface. The first model, also proposed to explain $\mathrm{Na}^{+}$ion emission from $\mathrm{NaCl}$ (001), involves the emission of a cation from a site adjacent to a surface $\mathrm{F}$ center, thereby forming a surface $P$ center. The second model involves the emission of a cation that is initially adsorbed atop the surface $F$ center. The relative energetics of $\mathrm{Mg}^{+}$and $\mathrm{Mg}^{++}$emission from different defect sites at the (001) surface have been computed with DFT, in the local density approximation. Preliminary values for the kinetic energies of $\mathrm{Mg}^{+}$and $\mathrm{Mg}^{++}$ions in the second model are found to be $1.15 \mathrm{eV}$ and $5.99 \mathrm{eV}$, in rough agreement with the experimental values of $2.0 \mathrm{eV}$ and $6.7 \mathrm{eV}$, respectively. 


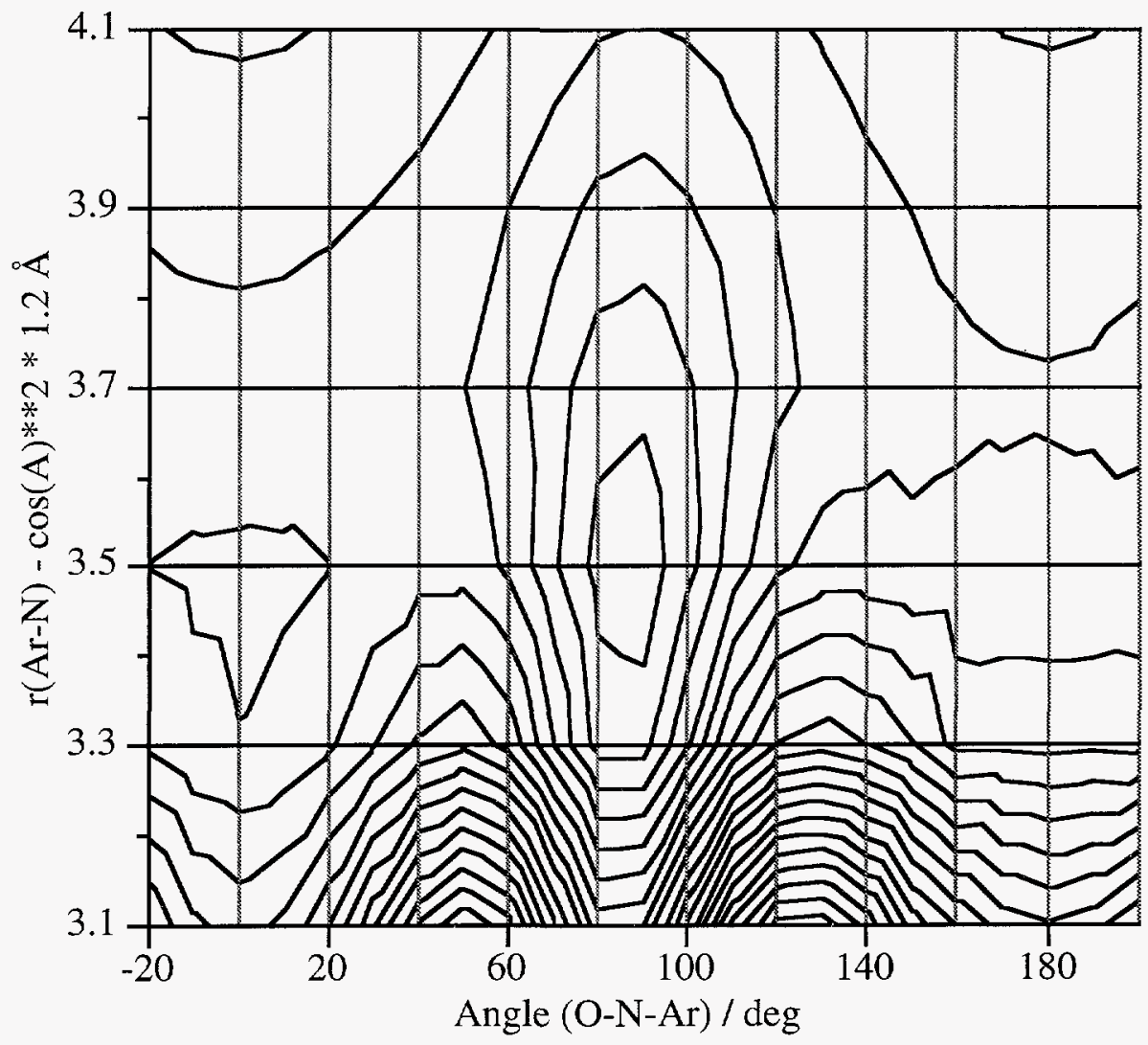

Figure 4.12. The energy surface of Ar in the field of a fixed NNO molecule.

\section{References}

1 R. J. Harrison, Int. J. Quant. Chem. 40, 337 (1991).

2 J. Boyle, Portable Programs for Parallel Processors (Holt, Rinehart and Winston, Inc., New York, New York, 1987)

3 MPI: A Message Passing Interface, by The Message Passing Forum in Supercomputing ' 93 (IEEE Computer Society Press, Los Alamitos, California, 1993), pp. 878.

4 R. A. Kendall, R. J. Harrison, R. J. Littlefield, and M. F. Guest, in Reviews in Computational Chemistry, edited by K. B. Lipkowitz and D. B. Boyd (VCH Publishers, Inc., New York, 1994).

5 M. E. Colvin, C. L. Janssen, R. A. Whiteside, and C. H. Tong, Theo. Chim. Acta 84, 301 (1993).

6 T. R. Furlani and H. F. King, J. Comp. Chem., 16, 91 (1993).

7 Global Arrays: A Portable Shared Memory Programming Model for Distributed Memory Computers, by J. Nieplocha, R. J. Harrison, and R. J. Littlefield, in Supercomputing '94 (IEEE Computer Society Press, Washington, D. C., 1994).

8 M. Arango, D. Berndt, N. Carriero, D. Gelernter, and D. Gilmore, Supercomputer Review 3(10) (1990).
M. Schuler, T. Kovar, H. Lischka, R. Shepard, and R. J. Harrison, Theo. Chim. Acta 94, 489 (1993). Symp. 29, 475 (1995).

11 A. P. Rendell, M. F. Guest, and R. A. Kendall, J. Comp. Chem. 14, 1429 (1993).

E. R. Jessup, Ph.D. thesis, Yale University, 1989.

3 S. S. Lo, B. Phillipps, and A. Sameh, SIAM J. Sci. Stat. Comput. 8(2) (1987).

14 Parallel Inverse Iteration with Reorthogonlization, by G. Fann and R. Littlefield, in Sixth SIAM Conference on Parallel Processing for Scientific Computing (SIAM, Philadelphia, Pennsylvania1993), pp. 409.

15 E. Anderson, Z. Bai, C. Bischof, J. Demmel, J. Dongarra, J. Du Croz, A. Greenbaum, S. Hammarling, A. McKenney, S. Ostrouchov, and D. Sorensen, LAPACK Users' Guide (Society for Industrial and Applied Mathematics, Philadelphia, 1992).

16 M. Dupuis, D. Spangler, and J. J. Wendolowski, HONDO (University of California, Berkeley, 1980). M. W. Schmidt et. al., QCPE Bull. 7, 115 (1987).

18 M. F. Guest, R. J. Harrison, J. H. v. Lenthe, and L. C. H. v. Corler, Theo. Chim. Acta 71, 117 (1987). 
M. F. Guest and et. al., (EPSRC Daresbury Laboratory, Daresbury, Warrington WA44AD, UK, 1991).

M. J. Frisch, G. W. Trucks, M. Head-Gordon, P. M. W. Gill, M. W. Wong, J. B. Foresman, B. G. Johnson, H. B. Schlegel, M. A. Robb, E. S. Replogle, R. Gomperts, J. L. Andres, K. Raghavachari, J. S. Binkley, C. Gonzalez, R. L. Martin, D. J. Fox, D. J. Defrees, J. Baker, J. J. P. Steward and J. A. Pople, , Revision C ed. (Gaussian, Inc., Pittsburgh, Pennsylvania, 1992).

M. Feyereisen, G. Fitzgerald, and A. Komornicki, Chem. Phsy. Lett. 208, 359 (1993). Washington, 1977. $218(1978)$

G. B. Bacskay, Chem. Phys. 61, 385 (1982).

Auxiliary Basis Expansions - The End of Four-Center Integral?, by J. Almlof in West Coast Theoretical Chemistry Conference (Sandia National Laboratory, Livermore, California, 1994)

O. Vahtras, J. Almlof, and M. Geyereisen, Chem. Phys. Lett. 213, 514 (1993).

J. Almlof and P. R. Taylor, in Advanced Theories and Computational Approaches to the Electronic Structure of Molecules, edited by C. Dykstra (D. Reidel, Dordrecht, Hingham, Massachusetts1984).

P. A. Malmqvist, A. Rendell, and B. O. Roos, J. Phys Chem. 94, 5477 (1990).

P. Hohenberg and W. Kohn, Phys. Rev. B 136, 864 (1964),

W. Kohn and L. J. Sham, Phys. Rev. A 140, 1133 (1965). edited by K. Labanowski and J. W. Andzelm (SpringerVerlag, New York, New York, 1991), pp. 7.

I. Dunlap, J. W. D. Connolly, and J. R. Sabin, J. Chem Phys. 71, 3396 (1979).

. Connolly, and J. R. Sabin, J. Chem. Phys. 71, 4993 (1979).

S. H. Vosko, L. Wilk, and M. Nusaii, Can. J. Phys. 58, 1200 (1980).

C. Lee, W. Yang, and R. G. Parr, Phys. Rev. B 37, 785 (1988).

P. Pulay, Chem. Phys. Lett. 73, 393 (1980).

P. Pulay, J. Comp. Chem. 3, 556 (1982).

T. P. Hamilton and P. Pulay, J. Chem. Phys. 84, 5728 (1986).

A. Warshel and M. Levitt, J. Mol. Biol. 103, 227 (1976).

M. J. Field, P. A. Bash, and M. Karplus, J. Comp. Chem. 11, 700 (1990).

43 R. V. Stanton, D. S. Hartsough, and K. M. Merz, J. Phys. Chem. 97, 11868 (1993).

T. Wesolowski and A. Warshel, J. Phys. Chem. 98, 5183 (1994).

W. Wong, M. J. Frisch, and K. B. Wiberg, J. Am Chem. Soc. 113, 4776 (1991).

47 J. Gao and X. Xia, Science 258, 631 (1992).

K. A. Sharp and B. Honig, J. Phys. Chem. 94, 7684 (1990). Phys. Chem. 98, 11059 (1994). (1990).

51 A. St-Amant, Ph.D. thesis, Universite de Montreal, 1992.

52 R. Dovesi, C. Pisani, C. Roetti, M. Causa, and V. R Saunders, (Quantum Chemistry Program Exchange, Publication 577, Bloomington, Indiana, 1988).

53 R. Dovesi, V. R. Saunders, and C. Roetti, (Universit á di Torino, Torino, Italy, 1992).

54 N. M. Harrison and V. R. Saunders, (S.E.R.C. Daresbury Laboratory, Daresbury, 1994).

55 A. Geist and et. al., (Oak Ridge National Laboratory, 1994).

56 M. P. Allen and D. J. Tildesley, Computer Simulation of Liquids (Clarendon Press, Oxford, England 1987).

57 J. Guenot and P. A. Kollman, J. Comp. Chem. 14, 295 (1993).

58 K. Tasaki, S. McDonald, and J. W. Brady, J. Comp. Chem. 14, 278 (1993).

59 S. W. DeLeeuw, J. W. Perram, and E. R. Smith, Proc. Roy. Soc. A 373, 27 (1980)

60 A. W. Appel, SIAM J. Sci. Stat. Comput. 6, 85 (1985).

61 J. E. Barnes and P. Hut, Nature 324, 446 (1986).

62 L. Hernquist, Comp. Phys. Comm. 48, 107 (1988).

63 R. W. Hockney and J. W. Eastwood, Computer Simulation Using Particles (Adam Hilger, New York, 1989).

64 L. V. Dommelen and E. A. Rundensteiner, J. Comp. Phys. 83, 126 (1989).

65 L. Greengard and V. Rokhlin, J. Comp. Phys. 73, 325 (1987).

66 L. Greengard, The Rapid Evaluation of Potential Fields in Particle Systems (MIT Press, Cambridge, 1987).

67 H.-Q. Ding, N. Karasawa, and I. W. A. Goddard, J. Chem. Phys. 97, 4309 (1992).

68 R. Kutteh and J. B. Nicholas, Comp. Phys. Comm. 86, 227 (1995).

69 R. Kutteh and J. B. Nicholas, Comp. Phys. Comm. 86, 236 (1995).

70 H. Grubmuller, H. Heller, A. Windemuth, and $K$. Schulten, Mol. Sim. 6, 121 (1991).

71 W. B. Streett, D. J. Tildesley, and G. Saville, Mol. Phys. 35, 639 (1978). 
72

73

R. A. Kendall, T. H. Dunning Jr., and R. J. Harrison, J. Chem. Phys. 96, 6796 (1992).

74 D. E. Woon and T. H. Dunning Jr., J. Chem. Phys. 98, 1358 (1993).

75 J. A. Nichols, D. Heryadi, D. L. Yeager, and T. Golab, J. Chem. Phys. 100, 2947 (1994).

76 D. Yeager, J. Nichols, and J. Golab, J. Chem. Phys. 100, 6514 (1994).

77 D. Heryadi, D. L. Yeager, J. T. Golab, and J. A. Nichols, J. Chem. Phys. 102, 9444 (1995),

78 D. Heryadi, D. L. Yeager, J. T. Golab, and J. A. Nichols, Theo. Chim. Acta 90, 273 (1995).

79 J. P. Perdew, Phys. Rev. B 33, 8822 (1986).

801993 ASHRAE Handbook: Fundamentals, Vol. , edited by R. A. Parsons (American Society of Heating, Refrigerating and Air-Conditioning Engineers, Atlanta, 1993).

81

R. Ditchfield, Mol. Phys. 27, 789 (1974).

82 K. Wolinski, J. F. Hinton, and P. Pulay, J. Am. Chem. Soc. 112, 8251 (1990).

83 J. Gauss, J. Chem. Phys. 99, 3629 (1993).

84 T. A. Hu, E. L. Chappell, and S. W. Sharpe, J. Chem. Phys. 98, 6162 (1993). 

Productive use of the advanced computational resources available to molecular and environmental scientists requires not only a revolution in computational methods, but also a corresponding revolution in the tools for managing and analyzing complex computational experiments. The Computational Chemistry Environment Group is developing the Extensible Computational Chemistry Environment (ECCE') to begin to address these needs.

\section{Extensible Computational Chemistry Environment}

What is ECCE? ECCE', is an integrated, comprehensive environment for molecular modeling and simulation. Key components are applications with graphical user interfaces (GUI), chemistryspecific visualization applications, persistent databases, resource management, and scientific data management. The GUI assists in the selection of computational parameters, by recasting database input queries in terms of scientific requirements, and assisting the user via chemistry-specific help facilities. Visualization tools focus on graphical manipulation and analysis of data from molecular modeling calculations. Seamless integration of tools is accomplished through a common architecture for molecular modeling applications that is based on an object-oriented molecular and computational experiment management data model. The intent is to allow the execution of complex computational "experiments," to provide a framework for extending the computational resources in the systems, and to facilitate information sharing. ECCE' also is built on publicly and commercially available software: Molecular Inventor (Silicon Graphics Inc.) an object-oriented visualization toolkit, ObjectStore (Object Design Inc.) an objectoriented database management system, TeleUse (Thompson Software Products) a GUI development tool, and ToolTalk (Sun Microsystems, Inc.) for interprocessing communication.
The ECCE' design utilizes the data centered approach for scientific analysis. The framework (Figure 5.1) consists of an application desktop, a suite of visualization applications, a persistent database management system, and a mechanism to access various other resources via the internet. The ease of use is facilitated by GUIs. The emphasis is on providing/developing an environment for the user that enhances usability, productivity, software adaptability, system reliability, code extensibility, and maintainability.

ECCE' Design Objectives. There are many challenges in developing a complex extensible environment such as ECCE'. The complex nature of research problems in the EMSL demands that the software be designed with the ever-changing requirements of the users. To begin, the ECCE' development team interviewed over seventy potential users. The focus of the interviews was on the process of scientific research. The details of this study will be published at a later date. It is sufficient here to summarize a few pertinent ECCE' design objectives that were developed from that study.

1. Provide a user customizable chemistry desktop that enables users to access any ECCE' tool. The desktop must support integration of (i.e., access to) non-ECCE' tools. The desktop and ECCE' tools will adhere to an ECCE' style that is built upon industry standards and will be designed to work in a heterogeneous environment of desktop workstations.

2. Design all ECCE' tools to work with an underlying chemistry and experiment data model. This approach will facilitate the integration of ECCE' tools to build reference data sets to facilitate data sharing, to provide a common

The work reported in this section was supported by the Environmental and Molecular Sciences Laboratory Project. 
design basis for all tools, and to build sophisticated tools for browsing and extracting data rather than recreating data sets that are expensive. By enabling better integration of different applications, we can build sophisticated tools to integrate steps in the scientific process.

3. Support a black-box (i.e., batch run) model of computations currently in use in EMSL and plan for the future integration of other such codes.

4. Support any computer servers available in the EMSL networked environment.

5. Design for extensibility (and thus longevity) in the following key areas:
- different types of computational chemistry (ab initio, molecular dynamics, etc.)

- different codes (each chemist has a favorite)

- other areas of chemistry besides computational physical chemistry (experimental, geochemical, etc.)

- dynamic network environment (resources and resource availability will be very dynamic).

Development of ECCE' is continuing with current focus on developing the alpha version to give prospective users of the system a glimpse of things to come. Future versions, specifically the beta version, is scheduled for completion in March 1997.

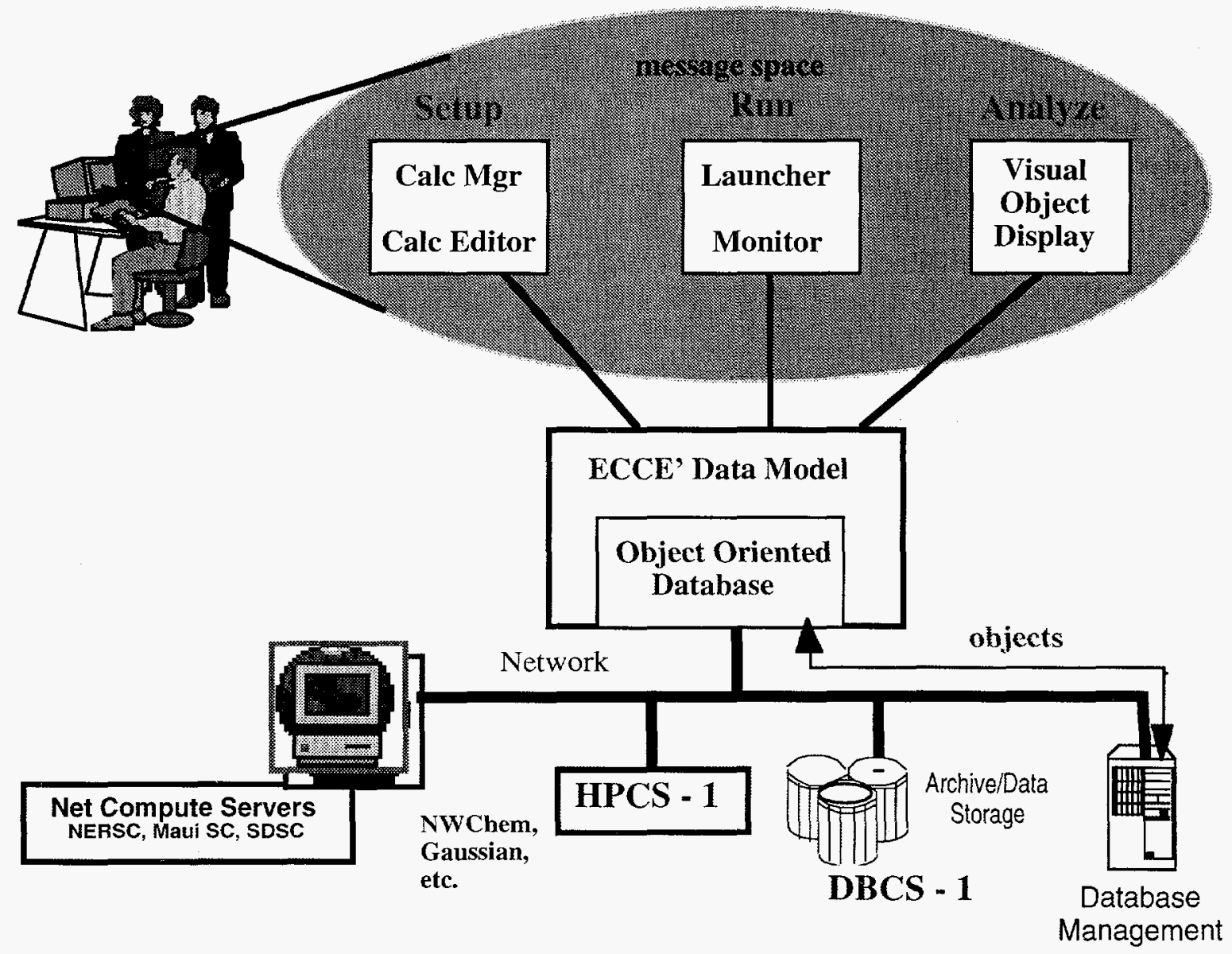

Figure 5.1. The ECCE'architecture consists of an application desktop, a suite of visualization applications, a persistent database management system, and a mechanism to access various other resources via the Internet. 
Future capability to be provided in ECCE's beta and 1.0 releases will be:

- Enhanced User Interface. We will have multiple viewing options on the experiment browser, such as icon view, text list view, tree outline view, etc.

- Advanced visualization techniques. We eventually will have tools to visualize data of higher than three dimensions. We will also have the capability of visualizing very large, complex data sets, such as molecular structures with thousands of atoms.

- Enhanced tools. Initially we are providing a molecule sketcher (ECCE' Sketch) for creating models of molecules or periodic systems. This tool will be enhanced to provide integrated geometry optimization.

- New tools. We will develop several additional tools once the initial ECCE' framework has been completed. Examples include a molecular orbital visualizer and a normal modes visualizer and analysis tool.

- Remote computation. We will develop a joblaunching tool that will facilitate remote computations. This tool will also provide capability for monitoring the progress of jobs.

- User extensibility. Eventually, ECCE' will be extensible by the end users.

- Extend data model. We will extend our data model to address a broader range of chemistry data sets, such as force field data sets.

\section{Architecture for Molecular Modeling Applications}

Object-Oriented Experiment Management and Computational Chemistry Data Model (T. L. Keller, K. L. Schuchardt, E. Overton, D. Feller, and D.R. Jones)

The foundation of $\mathrm{ECCE}^{\prime}$ is an underlying, common object-oriented data model and database management system. The EMSL data model serves as the common data format, allowing inter-application sharing of data, transparent persistence, and distribution. In 1994-95 we completed a framework of the EMSL data model composed of a chemistry data model and a computational experiment management data model. Contained in the framework of the EMSL data model are reusable and extensible components for complex chemical systems and generic tracking of processes and experiments that have been conducted. From the initial framework, we have extended both the chemistry and experiment management data models. Figure 5.2 provides an overview of the current EMSL data model in ECCE'. Showing both the chemistry and experiment management data model and their relationship to each other.

The chemistry data model is capable of handling periodic and non-periodic chemical systems. Non-periodic chemical systems are used to store traditional molecules and allow molecules to be constructed from smaller fragments. Periodic chemical systems are currently used to model crystals (three-dimensional periodic systems) but are also able to model one- and two-dimensional periodic systems. In addition to handling chemical systems, the chemistry data model also provides access to computational chemistry parameters. In particular, parameters for $a b$ initio computational chemistry (e.g., basis sets) are provided. Also, we provide access to a persistent, shared Periodic Table of the Elements.

The experiment management portion of the EMSL data model will eventually be used to store and manage enough information about computational experiments so that they may be reproduced. The EMSL data model currently models $a b$ initio computational chemistry experiments that are used to produce energies, optimized geometries, and vibrational frequency information. This experiment management information, in addition to the basis sets provided in the chemistry data model, is used by a basis sets advisor tool to intelligently guide naive users in setting up computational experiments.

\section{Periodic Table Graphical User Interface Widget (G.D. Black)}

A Periodic Table user interface toolkit primitive or widget was completed for EMSL graphical user interface development and ECCE' applications in particular. Figure 5.3 shows the Periodic 


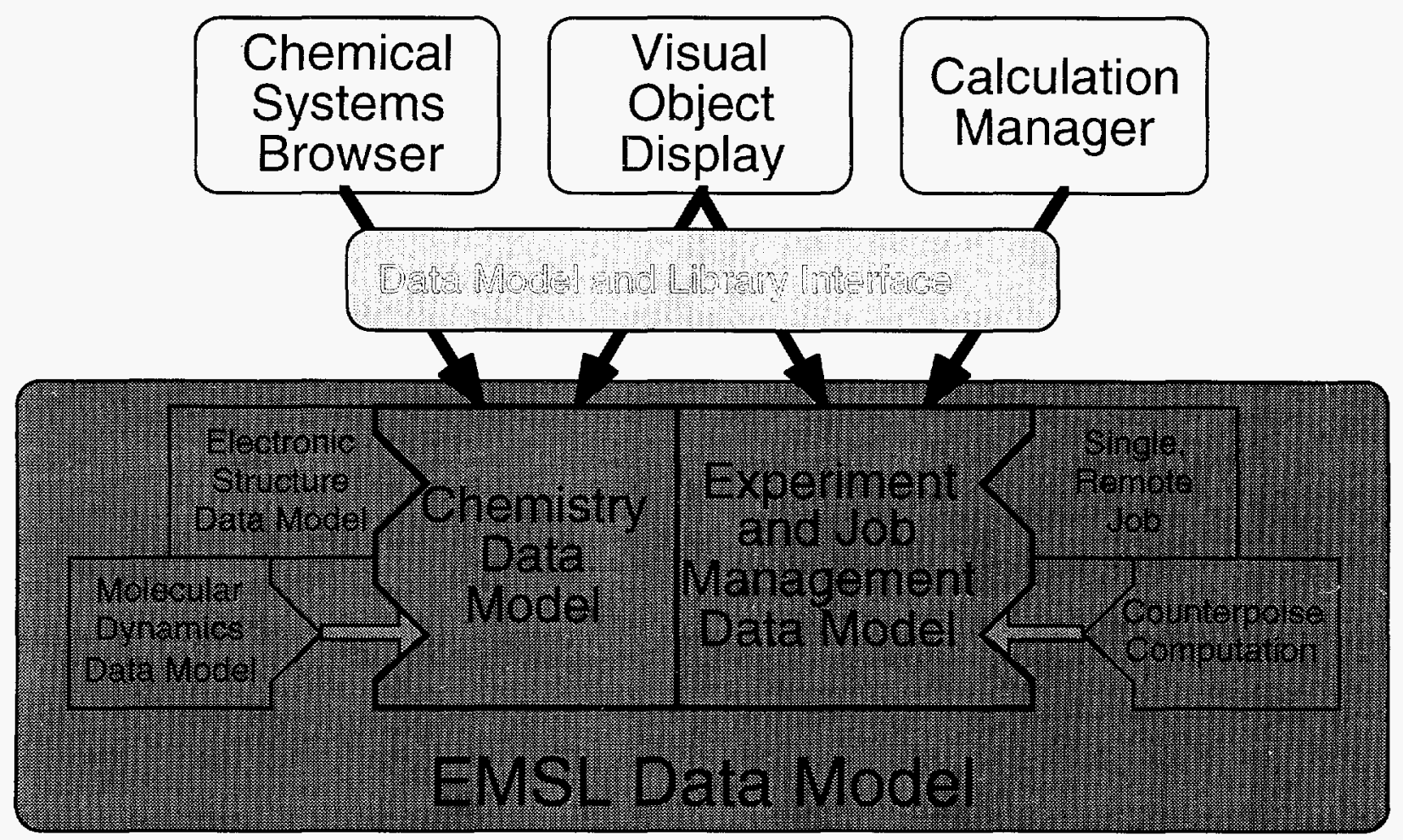

Figure 5.2. The extensible chemistry data model and experiment management data model as designed in ECCE'.

Table widget in a sample application. The widget is required for applications in which the user must select single or multiple chemical elements. An example of an application that will use the periodic table widget is the ECCE' periodic system builder. The chemist selects an element in the periodic table as if it were a drawing palette so that all atoms created in the builder are that element until another element is selected.

Another ECCE' application that uses the Periodic Table widget is the basis set utility. Here, selecting elements in the table narrows the list of possible basis sets to those supporting all selected elements. The Periodic Table widget is also valuable in applications that display correlations between elements and other criteria. For the basis set utility, this criteria is whether an element is supported by the currently selected basis set. Allowing user selections independent of displaying application correlations lets chemists work from both ends of the problem at the same time in selecting a basis set. Either a basis set is selected and all elements supported by the basis set are shown or the elements are selected and the list of applicable basis sets is restricted. The technique implemented to display application correlations allows up to four correlations simultaneously for the Periodic Table by using colored markers in element corners.

The Periodic Table widget is available through the Neuron Data Open Interface cross platform GUI builder, OpenEdit. The OpenEdit widget palette contains an icon for the Periodic Table. Selecting the icon allows an instance of the periodic table widget to be placed and sized on an Open Interface window. A custom editor dialog for the initial attributes of the Periodic Table comes up by double clicking on the Periodic Table widget in OpenEdit. Thus, the widget is integrated into the Open Interface environment as if it were a standard widget supplied by Neuron Data such as a push button. The Application Programmer's Interface (API) for the Periodic Table is written in $\mathrm{C}++$ and follows the design of other Open Interface widgets.

The user interacts with the widget by selecting individual elements with the left mouse button. 


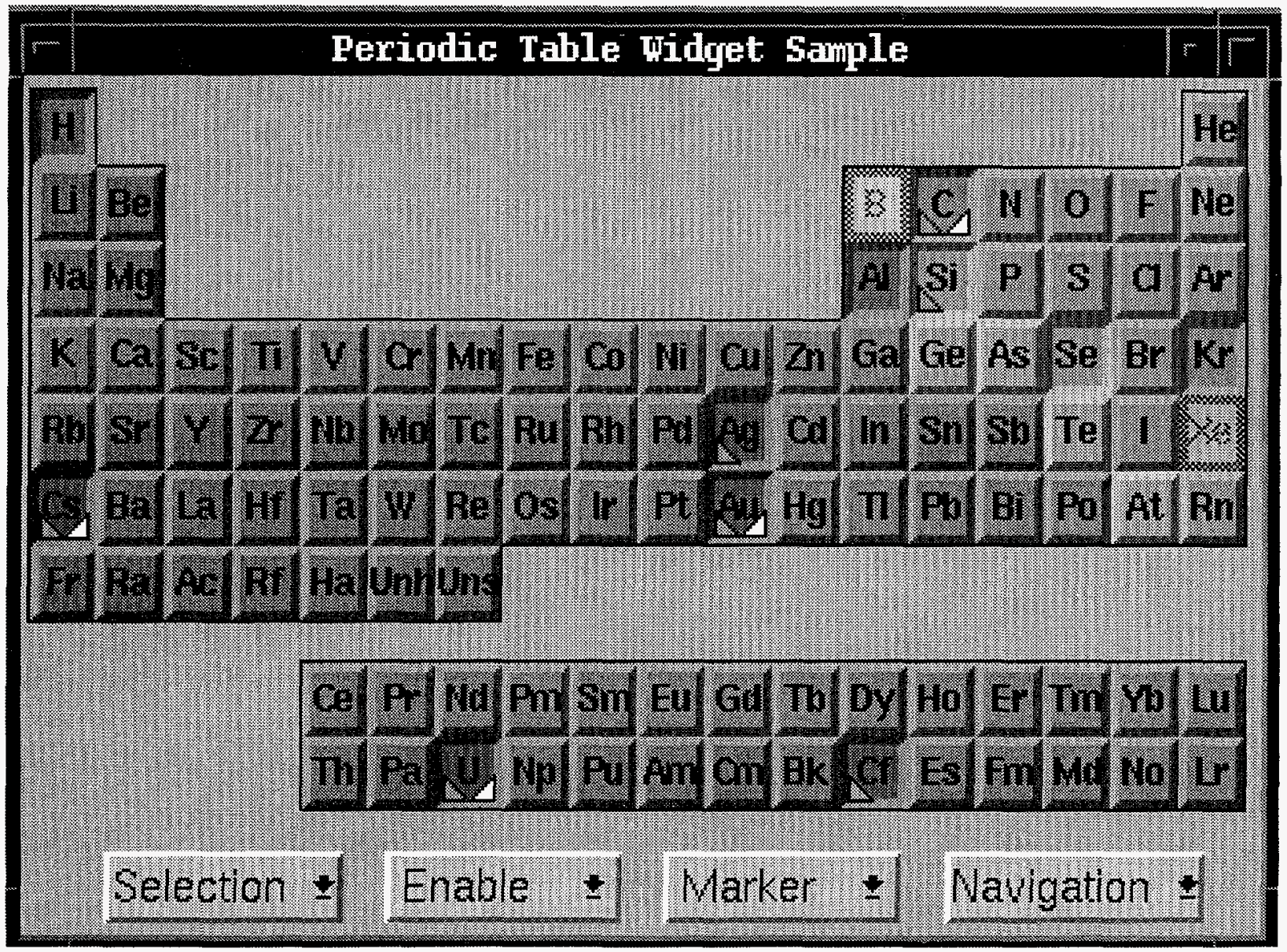

Figure 5.3. Periodic Table widget in a sample application

Selected elements are indicated by a "pushed- in" three-dimensinal visual effect. The right mouse button displays a popup menu with standard items that let the user set the Periodic Table element color scheme (e.g., by physical state or metallic properties), and show atomic numbers for elements in addition to symbols. Custom items for the menu can be added by the user interface developer for application-specific actions. Applications developed within ECCE' automatically have access to additional menu items for displaying a read-only dialog with elemental data and to color the elements based on a chemical system visualization displayed in another window. Additionally, an application could use a custom menu item, for instance, to allow the user select parameter values through a dialog where the parameters are associated with individual elements.
Internet Access to the EMSL Gaussian Basis Set Library (K. L. Schuchardt, D. Feller, and D. R. Jones)

In late June of 1994, the EMSL announced the availability of its first internet application, the Gaussian Basis Set Library. ${ }^{\dagger}$ Within several months, scientists from around the world with interest in $a b$ initio electronic structure methods logged several thousand accesses to our library through the EMSL home page. In November 1995 , this application was recognized as one of the Best-of-the-Web applications by Chemical $\mathcal{E}$ Engineering News. Figure 5.4 shows an example of the WWW page for the Gaussian Basis Set Library. The utility allow users to extract one or

The URL for the Gaussian Basis Set Library is http://www.emsl.pnl.gov:2080/forms/basisform.html and is accessible from the EMSL home page (URL http:/ /www.emsl.pnl.gov:2080). 


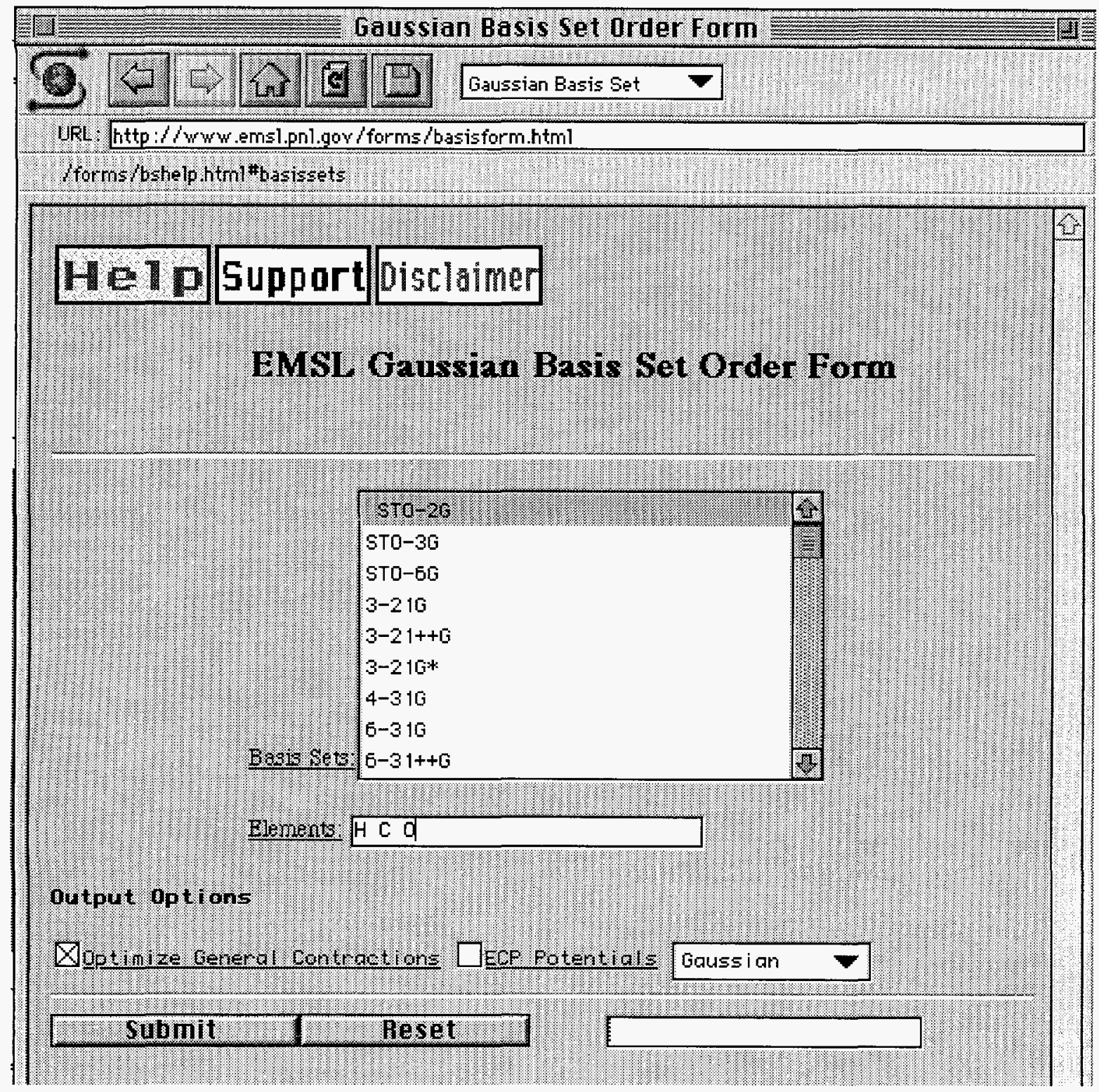

Figure 5.4. World Wide Web page for the Gaussian Basis Set Library.

more of the basis sets in the library in a format appropriate for some of the most widely used $a b$ initio codes (e.g., Gaussian, GAMESS, HONDO, etc.). Auxiliary information related to each basis set, such as the numbers and types of functions, literature citations, and graphical representations, are also available.

By the end of 1994, the library that is available externally contained over 60 basis sets, including the latest correlation consistent sets developed by Dunning and coworkers at the EMSL, and many effective core potentials. The internal EMSL library contained over 90 basis sets. As EMSLdeveloped sets appear in print, they are moved from the internal to the external library. Basis sets are constantly being added and suggestions are welcomed.

In the future, the Computational Chemistry Environment and High Performance Computing groups in the EMSL plan to offer a variety of user interface, visualization, and parallel application software for researchers doing computational 
chemistry. As part of that work, an advanced basis set utility is currently being implemented for UNIX workstations. This Basis Set Tool will support very flexible basis set requests and will offer users optional advice on which basis sets might be most appropriate for their needs. All of the tools, such as the one just described, will be integrated into $\mathrm{ECCE}^{\prime}$. $^{+}$

EMSL Graphics and Visualization Laboratory (R. May and D. R. Jones)

The EMSL Graphics and Visualization Laboratory (GVL) will house a multimedia/video workstation as well as high performance graphics system. The multimedia/video station is to serve as the central point for collection, processing, and distribution of multimedia and video products. The use of multimedia technology for presentations, training, and collaborative efforts is rapidly growing. Part of our task is to provide support for these capabilities in the EMSL GVL.

The use of multimedia and video in scientific visualization has several applications. First video tapes of animations are used in support of presentations or are used for demonstrations when it is not convenient to run the actual software. Multimedia authoring packages are also being used to develop mockups or proof of concept for software capabilities. These high level authoring packages provide an effective means to show the look, feel, and proposed capabilities of a software package without having to build a complete prototype. Finally, animation, images, audio, text (slides), and data can be stored on portable digital media such as hard drives or CD-ROMs. This material can be used by authoring packages for development of a complete interactive presentation for distribution.

Currently the Macintosh is the preferred platform for general multimedia and image processing work. The Macintosh provides an entire suite of multimedia, image processing, and video processing software and hardware. The interim EMSL GVL has obtained a PowerPC 8100/80 AV.

+ For information on either the Gaussian Basis Set Library or the upcoming Basis Set Tool (Unix software), contact Don Jones (dr_jones@pnl.gov), David Feller (df_feller@pnl.gov) or Karen Schuchardt (kl_schuchardt@pnl.gov).
This system is being used to evaluate the current capabilities of multimedia systems as well as help to identify what capabilities will be needed by the EMSL research staff.

We are also looking into using a UNIX-based multimedia/video workstation. There are several potential advantages to a UNIX-based workstation. The high-performance graphics workstations, which will be used to supply animations for the multimedia station, will be UNIX systems. Traditionally, UNIX-to-UNIX network connectivity has been more robust than UNIX-to-PC connectivity. Additionally, the video industry is moving to fully digital video UNIX-based servers. Companies such as AVID and Sony are today developing these capabilities to reduce the need for analog editing and storage of video. UNIX workstations provide the necessary hardware, expandability, and mass storage capabilities required by these systems. By going to a digital solution, fewer analog editing and storage devices will be required and other hardware such as wave form vector scopes can be replaced by software solutions. If animations can be archived in digital form, then higher quality can be maintained without the generational loss associated with analog media.

While UNIX systems have had video and image processing capabilities for some time, they have lacked multimedia authoring capabilities. With the continued expanding use of multimedia applications, manufacturers of UNIX workstations are beginning to realize the importance of having multimedia authoring software for their platforms. Both Sun and HP now have multimedia authoring software for their systems and SGI is working with Macromedia to port the popular Macintosh Director software over to the SGI platform. Continued improvements in these areas will help to produce fully capable UNIX multimedia/video workstations in the near future. 



\section{Publications and Submissions} January 1, 1994 - December 31, 1995

Theory, Modeling, and Simulation

\section{Publications}

1994

GE Arnold and RL Ornstein. "An Evaluation of Implicit and Explicit Solvent Model Systems for the Molecular Dynamics Simulation of Bacteriophage T4 Lysozyme," PROTEINS: Structure, Function, and Genetics 18, 19 (1994).

JL Anchell, JC White, MR Thompson, and AC Hess. "An Ab Intitio Peridoic Hartree-Fock Study of Group IA Cations in ANA Type Zeolites," J. Phys. Chem. 98, 4463 (1994).

LX Dang. "Free Energies for Association of $\mathrm{Cs}^{+}$to 18-Crown-6 in Water: A Molecular Dynamics Study Including Counter Ions," Chem. Phys. Lett. 227, 211 (1994).

LX Dang. "Potential of Mean Force for the Methane Pair in Water," J. Chem. Phys. 100, 9032 (1994).

G. de Brouckère, D. Feller, and J. Brion. "Configuration Interaction Calculations on the $\mathrm{P}_{2}$ Molecule. I. Potential Energy Curves of the $X^{1} \Sigma_{\mathrm{g}}{ }^{+}$and a ${ }^{3} \Sigma_{\mathrm{u}}{ }^{+}$States of the P2. Spectroscopic Properties," J. Phys. B. 27, 1657 (1994).

S Deshmukh, JD Myers, SS Xantheas, and WP Hess. "Investigation of Acetyl Chloride Photodissociation by Photofragment Imaging," Journal of Physical Chemistry 98, 12535 (1994).

D Feller,ED Glendening, RA Kendall, and KA Peterson. "An Extended Basis Set Ab Initio Study of $\mathrm{Li}^{+}\left(\mathrm{H}_{2} \mathrm{O}\right)_{\mathrm{n}, \mathrm{n}=1}$ to 6," J. Chem. Phys. 100, 4981 (1994).

H Gai and BC Garrett. "Path Integral Calculations of the Free Energies of Hydration of Hydrogen Isotopes (H, D, and Mu)," J. Phys. Chem. 98, 9642 (1994).

BC Garrett and GK Schenter. "Variational Transition State Theory for Activated Chemical Reactions in Solution," Int. Rev. Phys. Chem. 13, 263 (1994).

BC Garrett and GK Schenter. "Nonequilibrium Solvation for an Aqueous-Phase Reaction: Kinetic Isotope Effects for the Addition of Hydrogen to Benzene," ACS Symposium Series Volume 568, 122 (1994).

ED Glendening, D Feller, and MA Thompson. "An Ab Initio Investigation of the Structure and Alkali Metal Cation Selectivity of 18-crown-6," J. Am. Chem. Soc. 116, 10657 (1994).

AC Hess, MI McCarthy, and PF McMillan. "Ab initio Methods in Geochemistry and Mineralogy," in Advances in Electronic Structure Theory, T.H. Dunning, Jr. (ed), JAI Press, New York (1994).

JE Jaffe, NM Harrison, and AC Hess. "Ab Initio Study of ZnO 1āo Surface Relaxation," Phys. Rev. B 49, 11153 (1994).

GL Keldson, JB Nicholas, KA Carrado, and RE Winans. "Molecular Modeling of the Enthalpies of Absorption of Hydrocarbons on Smectite Clay," J. Phys. Chem. 98, 279 (1994). PNL-SA-22771. 
MI McCarthy and NM Harrison,. "Ab Initio Determination of the Bulk Properties of MgO," Phys. Rev. B49, 8574 (1994).

M Messina, BC Garrett, and GK Schenter. "Variational Solutions for the Thermal and Real Time Propagator Using the McLachlan Variational Principle," J. Chem. Phys. 100, 6570 (1994).

RJ Miller and D Feller. "A Comment on the ${ }^{2} \Pi$ Ground State of Nitric Oxide," J. Phys. Chem. 98, 10375 (1994).

JB Nicholas and AC Hess. "Ab initio Periodic Hartree-Fock Investigation of a Zeolite Acid Site," J. Am. Chem. Soc. 16, 5428 (1994).

R Pandey, JE Jaffe, and NM Harrison. "Ab Initio Study of High Pressure Phase Transition in GaN," J. Phys. Chem. Solids 55, 1357 (1994).

MD Paulsen and RL Ornstein. "Active Site Mobility Inhibits Reductive Dehalogenation of 1,1, 1Trichloroethane," J. Comp.-Aided Molecu. Design 8, 389 (1994).

KA Peterson, DE Woon, and TH Dunning, Jr. "Benchmark Calculations with Correlated Molecular Wave Functions. IV. The Classical Barrier Height of the $\mathrm{H}+\mathrm{H}_{2} \rightarrow \mathrm{H}_{2}+\mathrm{H}$ Reaction," J. Chem. Phys. 100, 7410 (1994).

CA Scamehorn, NM Harrison, and MI McCarthy. "Water Chemistry on Surface Defect Sites: Chemidissociation vs. Physisorption on MgO (001)," J. Chem. Phys. 101, 1547 (1994).

GK Schenter, G Mills, and H Jonsson. "Reversible Work Based Quantum Transition State Theory," J. Chem. Phys. 101, 8964 (1994).

RF Setlik, DJ Meyer, M Shibata, R Roskitalski, RL Ornstein, and R Rein. "A Full-Coordinate Model of the Polymerase Domain of HIV-I Reverse Transcriptase and Its Interaction with a Nucleic Acid Substrate," J. Biomolecu. Struct. Dyn. 12, 37 (1994).

DE Smith and LX Dang. "Computer Simulations of NaCl Association in Polarizable Water," J. Chem. Phys. 100, 3757 (1994).

DE Smith and LX Dang. "Computer Simulations of Cesium-Water Clusters: Do Ion-Water Clusters Form Gas-Phase Clathrates?" J. Chem. Phys. 101, 7873 (1994).

DE Smith and LX Dang. "Interionic Potentials of Mean Force for $\mathrm{SrCl}_{2}$ in Polarizable Water: A Computer Simulation Study," Chem. Phys. Lett. 230, 209 (1994).

MA Thompson, ED Glendening, and DF Feller. "The Nature of $\mathrm{K}^{+} /$Crown Ether Interactions: A Hybrid Quantum Mechanical-Molecular Mechanical Study," J. Phys. Chem 98, 10465 (1994).

MR Thompson, JR Ebner, JC White, JB Nicholas, J Anchell, and AC Hess. "A Concise Description of the Solid State Structure of Vanadyl Pyrophosphate and Implications for N-Butane Oxidation," Studies in Surface Science and Catalysis 82, 167 (1994).

DG Truhlar, Y-P Liu, GK Schenter, and BC Garrett. "Tunneling in the Presence of a Bath: A Generalized Transition State Theory Approach," J. Phys. Chem. 98, 8396 (1994). 
SS Xantheas and K Ruedenberg. "Potential Energy Surfaces of Carbon Dioxide," International Journal of Quantum Chemistry (Robert. G. Parr issue) 49, 409 (1994).

SS Xantheas. "Ab-initio Studies of the Cyclic Water Clusters $\left(\mathrm{H}_{2} \mathrm{O}\right)_{n}, n=1-6$ : II. Analysis of Many-Body Interactions," Journal of Chemical Physics 100, 7523 (1994).

SS Xantheas and TH Dunning, Jr. "Structures and Energetics of the $\mathrm{F}^{-}\left(\mathrm{H}_{2} \mathrm{O}\right)_{n^{\prime}} n=1-3$ Clusters from ab-initio Calculations," Journal of Physical Chemistry 98, 13489 (1994).

\section{5}

E Aprà. "Hartree-Fock Treatment of Spin-Polarized Crystals, in Quantum Mechanical Calculations of the Properties of Crystalline Materials," Lecture Notes in Chemistry Springer Verlag, Berlin, (1995).

DE Bernholdt and RJ Harrison. "Orbital Invariant Second-Order Many-Body Perturbation Theory on Parallel Computers: An Approach for Large Molecules," J. Chem. Phys., 102(24), 9582 (1995).

DE Bernholdt, E Aprà, HA Früchtl, MF Guest, RJ Harrison, RA Kendall, RA Kutteh, X Long, JB Nicholas, JA Nichols, HL Taylor, and AT Wong. "Parallel Computational Chemistry Made Easier: The Development of NWCHEM," Int. J. Quantum Chem. 29, 475 (1995).

RA Bradley, Jr., E Lanzendorf, MI McCarthy, TM Orlando, and WP Hess. "Molecular NO Desorption from Crystalline Sodium Nitrate by Resonant Excitation of the $\mathrm{NO}_{3}{ }^{-} \Pi-\Pi$ * Transition," Journal of Physical Chemistry, 99, 11715 (1995).

TM Chang, KA Peterson, and LX Dang. “Molecular Dynamics Simulations of Liquid, Interface, and Ionic Solvation of Polarizable Carbon Tetrachloride," J. Chem. Phys. 103, 7502 (1995).

LX Dang. "Mechanism and Thermodynamics of Ion Selectivity in Aqueous Solutions of 18-Crown-6 Ether: A Molecular Dynamics Study," J. Am. Chem. Soc. 117, 6954 (1995).

LX Dang and PA Kollman. "Free Energy of Association of the K+:18-Crown- 6 Complex in Water: A New Molecular Dynamics Study," J. Phys. Chem. 99, 55 (1995).

LX Dang and DE Smith. "Comment on 'Mean Force Potential for the Calcium-Chloride Ion Pair in Water' E. Guardia, A. Robinson and J.A. Pedro, J. Chem. Phys. 99, 4229 (1993)," J. Chem. Phys. 102, 3483 (1995).

H Dachsel and H Lischka. "An Efficient Data Compression Method for the Davidson Subspace Diagonalization Scheme," Theoretica Chimica Acta 92(6), 339 (1995).

TH Dunning, Jr. and SS Xantheas. "Effect of Solvation on Chemical Reactions. I. Addition of a Single Water Molecule to the $\mathrm{H}^{-}+\mathrm{H}_{2} \mathrm{O} \rightarrow \mathrm{OH}^{-}+\mathrm{H}_{2}$ Reaction," Journal of the Chinese Chemical Society ( $Y-T$ Lee Issue), 42, 241 (1995).

$G$ de Brouckére and DF Feller. "Configuration Interaction Calculations on the $P_{2}$ Molecule. II. Spectroscopic Properties of the $b^{3} \pi_{2, \mathrm{~g}}$ State, the $b^{3} \pi_{2, \mathrm{~g}^{-}} \mathrm{a}^{3} \Sigma_{\mathbf{u}}{ }^{+}$Transition Bands and the $\mathrm{a}^{3} \Sigma_{\mathbf{u}}{ }^{+}$State Revisited," Journal of Physics B, 28, 1393 (1995).

$\mathrm{G}$ de Brouckére and DF Feller. "Configuration Interaction Calculations on the $\mathrm{P}_{2}$ Molecule. III. Spectroscopic Properties of the $\mathrm{A}^{1} \pi_{\mathrm{g}}$ State, the $\mathrm{A}^{1} \pi_{\mathrm{g}}-\mathrm{X}^{1} \Sigma_{\mathrm{g}}{ }^{+}$Transition Bands and a Comparison with the Results for $\mathrm{X}^{1} \Sigma_{\mathrm{g}}{ }^{+}, \mathrm{a}^{3} \Sigma_{\mathrm{u}}{ }^{+}$and $\mathrm{b}^{3} \pi_{\mathrm{g}}$ States and Related Transition Bands," Journal of Physics B, 28, 3135 (1995). 
DF Feller, DE Woon, ED Glendening, and MF Feyereisen. "An Extended Basis Set Ab Initio Study of Alkali Metal Cation-Water Clusters," J. Chem. Phys. 103, 3526 (1995).

H Früchtl and P Otto. "A New Algorithm for the Evaluation of the Incomplete Gamma Function on Vector Computers," ACM Transactions on Mathematical Software, 20(4), 436 (1995).

H Gai, LX Dang, GK Schenter, and BC Garrett. "Quantum Simulations of Aqueous Ionic Cluster," $J$. Phys. Chem. 99, 13303 (1995).

ED Glendening and DF Feller. "Cation-Water Interactions: The $\mathrm{M}^{+}\left(\mathrm{H}_{2} \mathrm{O}\right)_{n}$ Clusters for Alkali Metals, $\mathrm{M}=\mathrm{Li}, \mathrm{Na}, \mathrm{K}, \mathrm{Rb}$, and Cs," J. Phys. Chem. 99, 3060 (1995).

ED Glendening, DF Feller, KA Peterson, EA McCullogh, Jr., and RJ Miller. "The Dipole Moment and Magnetic Hyperfine Properties of the Excited A2 (3ss) Ryberg State of Nitric Oxide," J. Chem. Phys. 103, 3517 (1995).

D Heryadi, D Yeager, J Golab, and J Nichols. "The Multiconfigurational Spin Tensor Electron Propagator Method (MCSTEP): Comparison with Extended Koopmans' Theorem Results," Theoretica Chimica Acta, 90, $273,(1995)$.

AC Hess, MI McCarthy, and PF McMillian. "Ab Initio Methods in Geochemistry and Mineralogy," Advances in Electronic Theory, JAI Press, New York(1995).

W-P Hu, GC Lynch, Y-P Liu, I Rossi, JJP Stewart, R Steckler, BC Garrett, AD Isaacson, D-H Lu, VS Melissas, and DG Truhlar. "Morate 6.5: A New Version of a Computer Program for Direct Dynamics Calculations of Chemical Reaction Rate," Computer Physics Communications, 88, 344 (1995).

RA Kendall, RJ Harrison, RJ Littlefield, and MF Guest. "High Performance Computing in Computational Chemistry: Methods and Machines," Reviews in Computational Chemistry, 6, 209 (1995).

R Kumble, GR Loppnow, S Hu, A Mukherjec, MA Thompson, and TG Spiro. "Studies of the Vibrational and Electronic Structure of the S1 Excited States of B-Substituted Porphyrins by Picosecond TimeResolved Resonance Raman Spectroscopy," Journal of Physical Chemistry, 99, 5809 (1995).

RA Kutteh and JB Nicholas. "Efficient Dipole Iteration in Polarizable Charged Systems Using the Dipole Cell Multipole Method and Application to Polarizable Water," Comp Phy Commun 86, 227 (1995).

RA Kutteh and JB Nicholas. "Implementing the Cell Multipole Method for Dipolar and Charged Dipolar Systems," Comp Phy Commun 86, 236 (1995).

RA Kutteh. "A Simpler and More Efficient Formulation of the Cell Multipole Method," CCP5 Quarterly, 43, (1995).

PJ Loida, SG Sligar, MD Paulsen, GE Arnold, and RL Ornstein. "Stereoselective Hydroxylation of Norcamphor by Cytochrome P450CAM: Experimental Verification of Molecular Dynamics Simulations," J. Biol. Chem. 270, 5326 (1995).

JI Manchester and RL Ornstein. "Enzyme-Catalyzed Dehalogenation of Pentachloroethane: Why F87WCytochrome P450cam is Faster than Wild-Type," Protein Engineering 8, 801 (1995). 
JI Manchester and RL Ornstein. "Molecular Dynamics Simulations Indicate that F87W, T185FCytochrome P450cam May Reductively Degalogenate 1,1,1-Trichloroethane," J. Biomol. Struct. \& Dyn. 13, 413 (1995).

MI McCarthy, NM Harrison, and MD Towler. "Ab Initio Study of the Surface and Interfacial Properties of a Layered MgO/NiO Film," Phys. Rev. B 52, 5375 (1995).

G Mills, H Jonsson, and GK Schenter. "Reversible Work Transition State Theory: Application to Dissociative Adsorption of Hydrogen," Surface Science 324, 305 (1995).

R Nada, C Pisani, and A.C. Hess. "Topological Defects at the (001) Surface of MgO: Energetics and Reactivity," Surface Science 336, 353 (1995).

JB Nicholas, J Fan, H Wu, SD Colson, and L-S Wang. "A Combined Density Functional Theoretical and Photoelectron Spectroscopic Study of Ge2O2." J. Chem. Phys. 102, 8277 (1995).

JB Nicholas and AC Hess. "Ab Initio Periodic Hartree-Fock Investigation of a Zeolite Acid Site," J. Am. Chem. Soc. 16, 5428 (1994).

JB Nicholas and MW Feyereisen. "An Evaluation of Correlation-Consistent Basis Sets in Calculating the Energetics and Structure of $\left(\mathrm{H}_{3} \mathrm{Si}\right) 20, \mathrm{H}_{3} \mathrm{SiOH}$, and $\mathrm{H}_{3} \mathrm{SiO}$," J. Chem. Phys. 103, 8031 (1995).

JA Nichols, D Heryadi, DL Yeager, and JT Golab. "The Multiconfigurational Spin Tensor Electron Propagator Method (MCSTEP): Comparison With Extended Koopmans' Theorem Results," Theor. Chim. Acta 90, 273 (1995).

Y Pak, RC Woods, and KA Peterson. "A Coupled Cluster Study of the Structure, Spectroscopic Properties, and Isomerization Path for $\mathrm{NCS}^{-}$and $\mathrm{CNS}^{-}$," Journal of Chemical Physics, 103, 9304 (1995).

BJ Palmer and JL Anchell. "Molecular Mechanics Parameters for Fluorine-Substituted Methanes from $a b$ initio Quantum Calculations," Journal of Physical Chemistry, 99, 12239 (1995).

MD Paulsen and RL Ornstein. "Dramatic Differences in the Motions of the Mouth of Open and Closed Cytochrome P450BM-3 by Molecular Dynamics Simulations," Proteins 21, 237 (1995).

KA Peterson. "Accurate Multireference Configuration Interaction Calculations on the Lowest ${ }^{1} \Sigma^{+}$and ${ }^{3} \Pi$ Electronic States of $\mathrm{C}_{2}, \mathrm{CN}^{+}, \mathrm{BN}$, and $\mathrm{BO}^{+}$, "Journal of Chemical Physics, 102, 262 (1995).

KA Peterson and TH Dunning, Jr., "Benchmark Calculations with Correlated Molecular Wave Functions. VII. The Structure and Binding Energy of the HF Dimer," Journal of Chemical Physics, 102, 2032 (1995).

KA Peterson and TH Dunning, Jr., "Intrinsic Errors in Several Ab Initio Methods: The Dissociation Energy of $\mathrm{N}_{2}$," Journal of Physical Chemistry, 99, 3898 (1995).

KA Peterson, Y Pak, and RC Woods. "A Coupled Cluster Study of the Structure, Spectroscopic Properties, and Isomerization Path for NCS- and CNS-", Journal of Chemical Physics, 103, 9304 (1995).

M Prencipe, A Zupan, R Dovesi, E Aprà, and VR Saunders. "An Ab Initio Study of the Structural Properties of LiF, NaF, KF, LiCl, NaCl and KCl," Phys. Rev. B 51, 3391 (1995).

SK Reese, SC Tucker, and GK Schenter. "The Reactive Flux Method in the Energy Diffusion Regime II Importance of the Solvent's Spectral Profile," J. Chem. Phys. 102, 104 (1995). 
GK Schenter, M Messina and BC Garrett. "A Variational Centroid Density Procedure for the Calculation of Transmission Coefficients for Asymmetric Barriers at Low Temperature," J. Chem. Phys. 103, 3430 (1995).

AP Smith, JK Wiggs, H Jonsson, H Yan, LR Corrales, P Nachtigall, and KD Jordan. "Si Adatom Binding and Diffusion on the Si(100) Surface: Comparison of Ab Initio, Semi-Empirical and Empirical Potential Results," J. Chem. Phys. 102, 1044 (1995).

MS Stave and JB Nicholas. "Density Functional Studies of Zeolites. 2. Structure \& Acidity of [T]-ZSM-5 Models (T = B, Al, Ga, and Fe)," J. Phys. Chem. 99, 15046 (1995).

R Steckler, W-P Hu, Y-P Liu, GC Lynch, BC Garrett, AD Isaacson, VS Melissas, D-H Lu, TN Truong, RN Rai, GC Hancock, JG Lauderdale, T Joseph, and DG Truhlar. "POLYRATE 6.5: A New Version of a Computer Program for the Calculations of Chemical Reaction Rates for Polyatomics," Computer Physics Communication, 88, 341 (1995).

MA Thompson. "Hybrid Quantum Mechanical/Molecular Mechanical Force Field Development for Large Flexible Molecules: A Molecular Dynamics Study of 18-Crown-6," J. Phys. Chem. 99, 4794 (1995).

MA Thompson. "A QM/MM Molecular Dynamics Study of the Potential of Mean Force for the Association of $\mathrm{K}^{+}$with Dimethyl Ether in Aqueous Solution," J. Am. Chem. Soc. 11711341 (1995).

MA Thompson and GK Schenter. "Excited States of the Bacteriochlorophyll b Dimer and Rhodopseudomonas viridis: A QM/MM Study of the Photosynthetic Reaction Center that Includes MM Polarization," J Phys. Chem. 99, 6374 (1995).

MD Towler, MI McCarthy, and NM Harrison. "Ab Initio Study of the Surface and Interfacial Properties of a Layered MgO/NiO Film," Physical Review B, 52, 5375 (1995).

AT Wong and RJ Harrison. "Approaches to Large-Scale Parallel Self-Consistent Field Calculations," J. Computational Chemistry 16, 1291 (1995).

SS Xantheas. "Ab-Initio Studies of Cyclic Water Clusters $\left(\mathrm{H}_{2} \mathrm{O}\right) \mathrm{n}, \mathrm{n}=1-6$ : III. Comparison of Density Functional with MP2 Results," J. Chem. Phys. 102, 4505 (1995).

SS Xantheas. "Theoretical Study of Hydroxide Ion-Water Clusters," J. Am. Chem. Soc. 117, 10373 (1995). PNL-SA-26477.

SS Xantheas and BT Sutcliffe. "The Hamiltonian for a Weakly-Interacting Trimer of Polyatomic Monomers," J. Chem. Phys. 103, 8022 (1995). 


\section{Other Publications}

JB Cushing, D Maier, M Rao, D Abel, DM DeVaney, and D Feller. “Computational Proxies: Modeling Scientific Applications in Object Databases," Proceeedings of the Scientific and Statistical Database Workshop, Charlottesville, Virginia, September(1994).

DR Jones, DF Feller, and KL Schuchardt. "Internet Access to the EMSL Gaussian Basis Set Library," Am. Chem. Soc. COMP Newsletter (1995).

D Maier, JB Cushing, D. Hansen, GD Purvis III, RA Bair, DM DeVaney, D Feller, and MA Thompson. "Object Data Models for Shared Molecular Structures," Computerized Chemical Data Standards: Databases, Data Interchange and Information Systems, ASTM STP 1214, R. Lysakowski and C. E. Gragg, (ed.), Am. Soc. for Testing and Materials, Philadelphia, Pennsylvania (1994). 